\title{
The transmembrane receptors Otk and Otk2 function redundantly in Drosophila Wnt signal transduction
}

\author{
PhD Thesis \\ in partial fulfilment of the requirements for the degree \\ "Doctor rerum naturalium (Dr. rer. nat.)" \\ in the Molecular Biology Program at the \\ Georg August University Göttingen, \\ Faculty of Biology
}

submitted by

Karen Linnemannstöns

born in

Braunschweig, Germany

2012 


\section{AFFIDAVIT}

I hereby declare that I prepared the thesis "The transmembrane receptors Otk and Otk2 function redundantly in Drosophila Wnt signal transduction" on my own with no other sources and aids than quoted.

Karen Linnemannstöns

Göttingen, December 14th 2012 


\section{PhD Thesis Committee}

Prof. Dr. Andreas Wodarz

Stem Cell Biology, Dept. of Anatomy and Cell Biology

Georg-August University, Göttingen

Prof. Dr. Annette Borchers

Functional Morphology and Evolution of Vertebrates

Philipps University, Marburg

Prof. Dr. Reinhard Schuh

Dept. of Molecular Developmental Biochemistry

Max Planck Institute for Biophysical Chemistry, Göttingen

Day of PhD examination: January 23rd, 2013 


\section{CONTENTS}

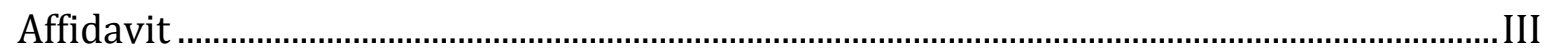

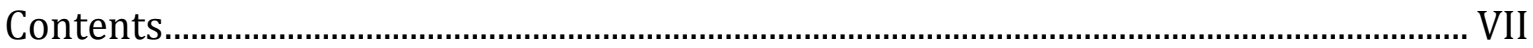

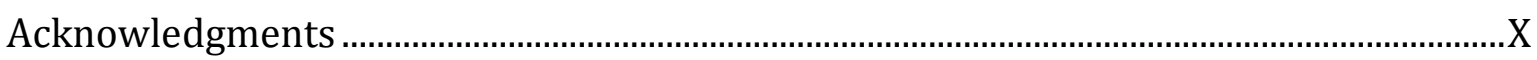

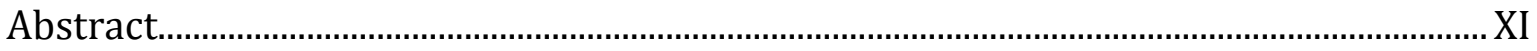

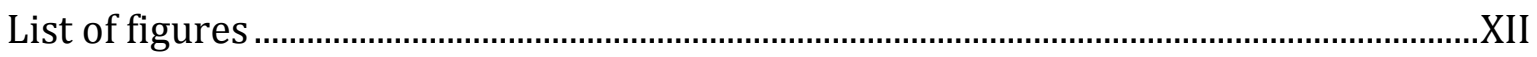

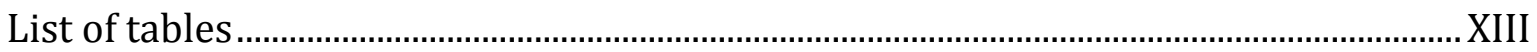

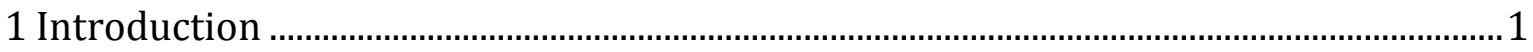

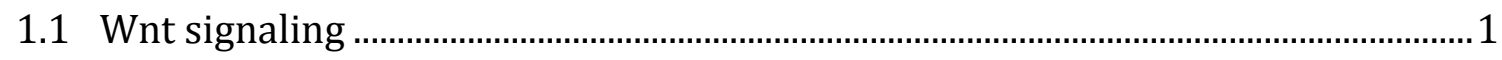

1.2 Canonical / $\beta$-catenin-dependent signaling …............................................................... 4

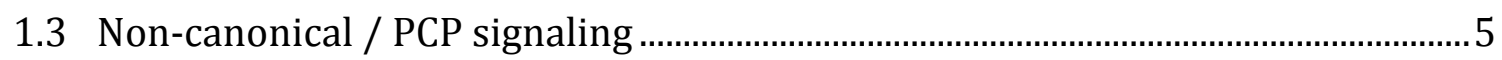

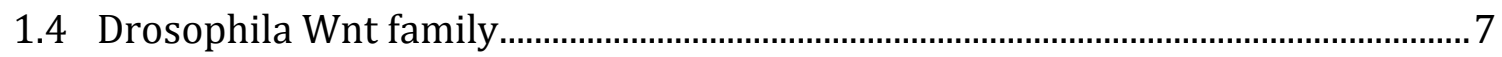

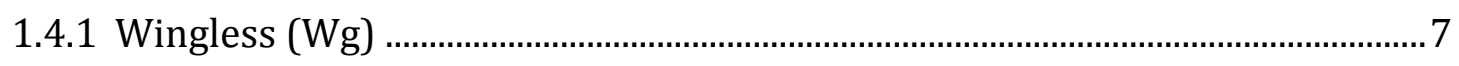

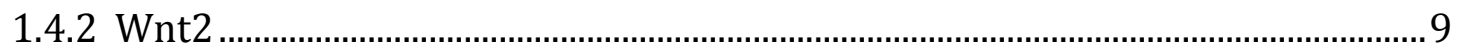

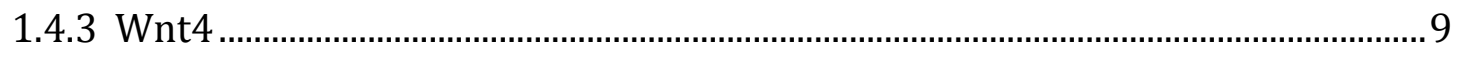

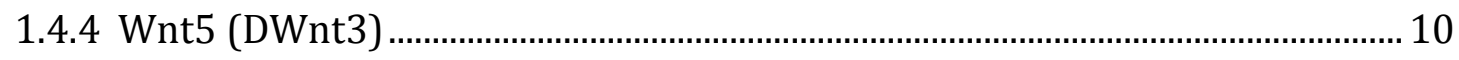

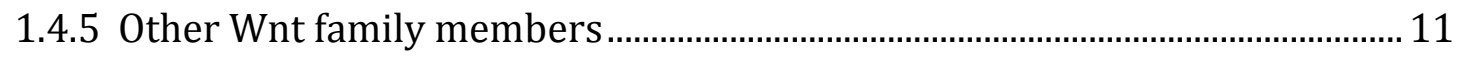

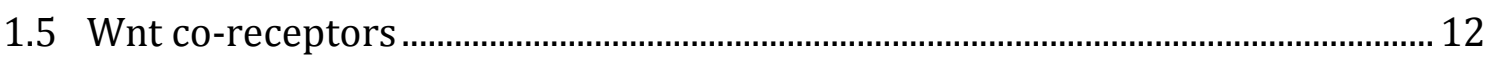

1.5.1 Modulation of Wnt signaling outcome by (co-)receptors................................... 13

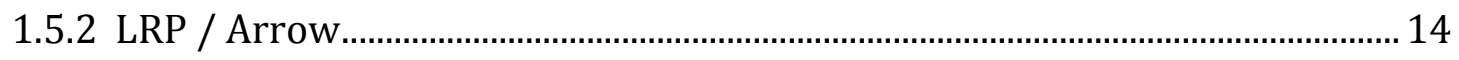

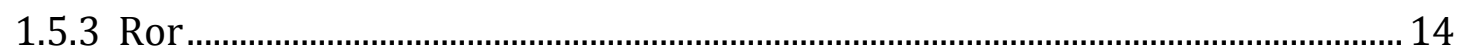

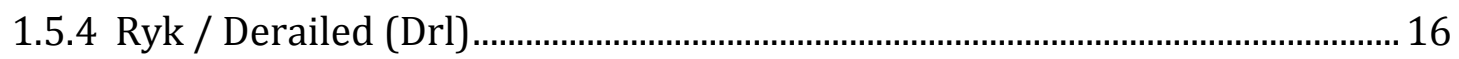

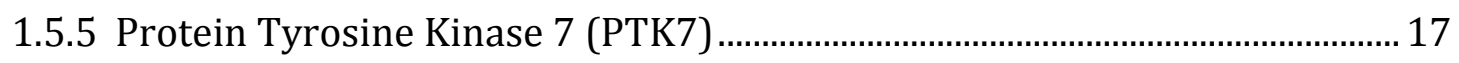

1.5.6 Off-track (Otk) - Drosophila homolog of PTK7 …............................................... 19

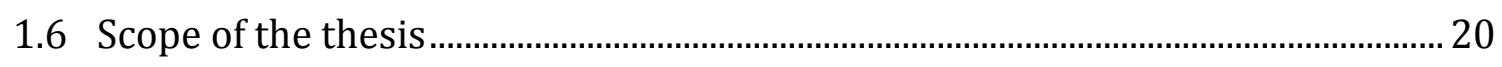

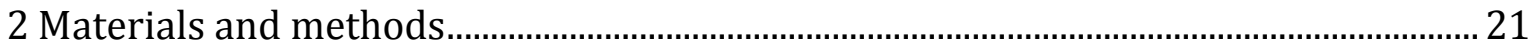

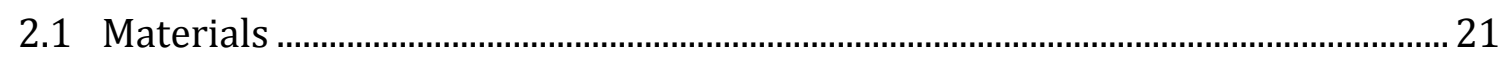

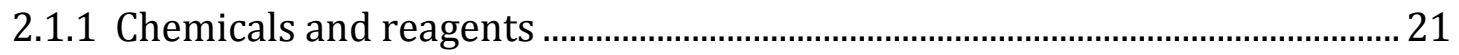

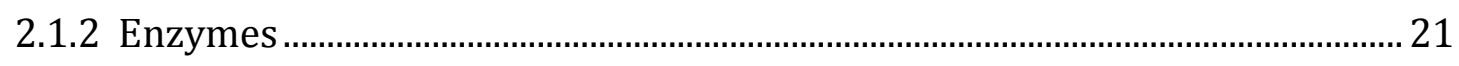

2.1.3 Bacterial strains ............................................................................................ 21

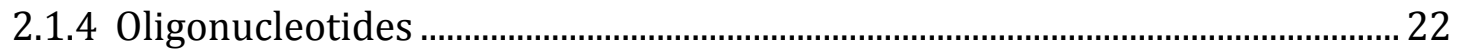

2.1.5 Vectors and constructs.................................................................................. 25

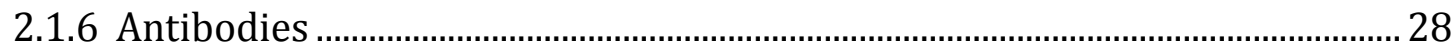

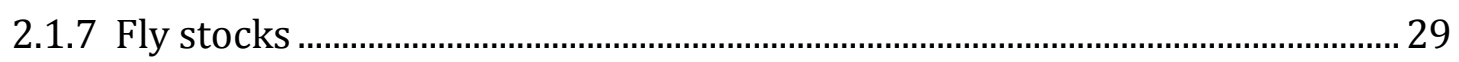

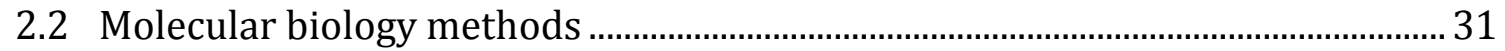

2.2.1 Polymerase chain reaction (PCR) …….......................................................... 31

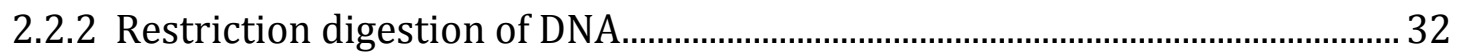

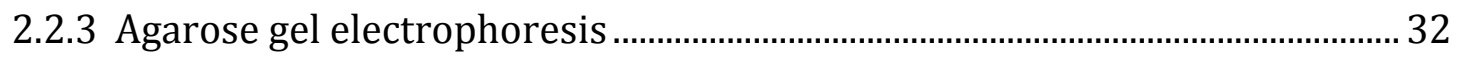

2.2.4 DNA extraction from agarose gels ........................................................................ 33

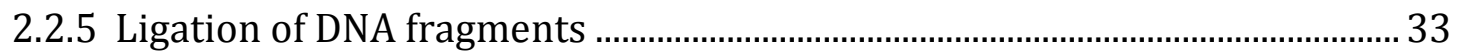


2.2.6 Generation of expression constructs by Gateway cloning technology ..........33

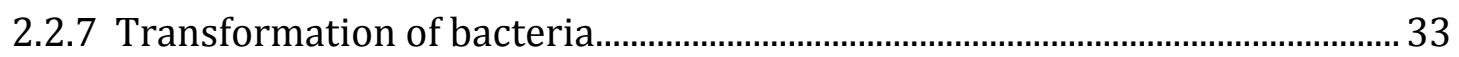

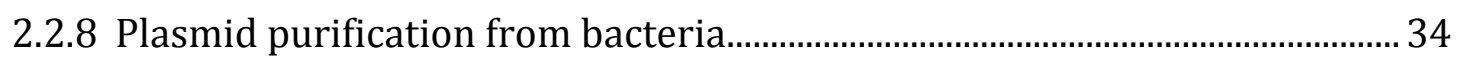

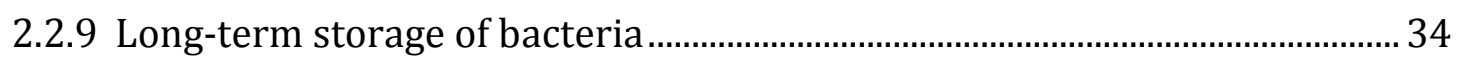

2.2.10 Site-directed mutagenesis …………......................................................... 34

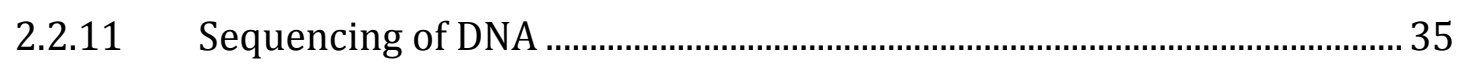

2.2.12 Extraction of genomic DNA from flies .......................................................... 36

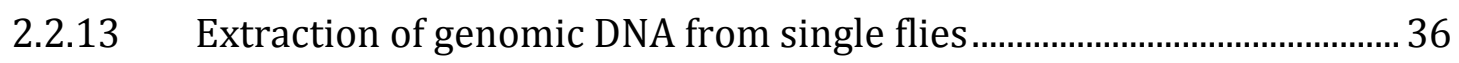

2.2.14 Long-template PCR ......................................................................................... 36

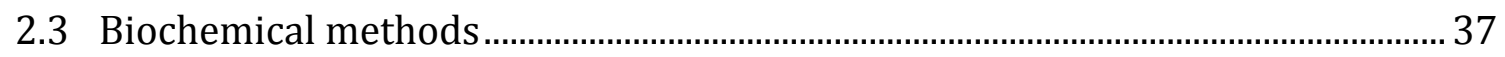

2.3.1 Culture and transfection of Schneider S2R+ cells........................................... 37

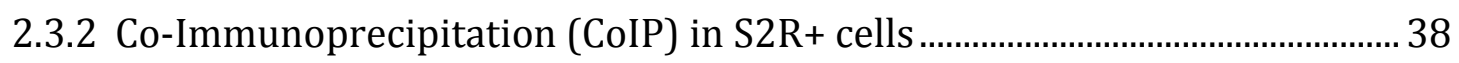

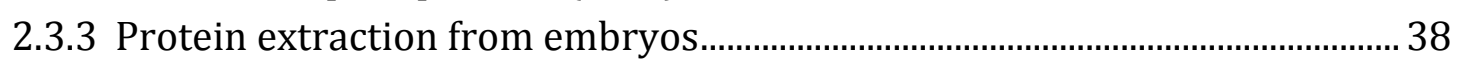

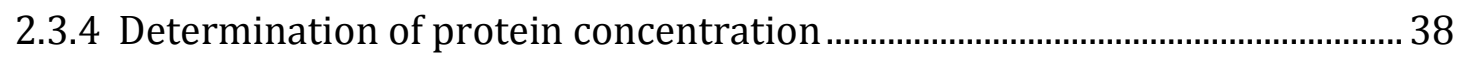

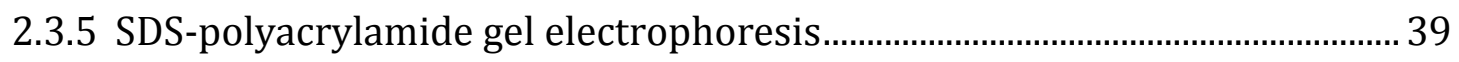

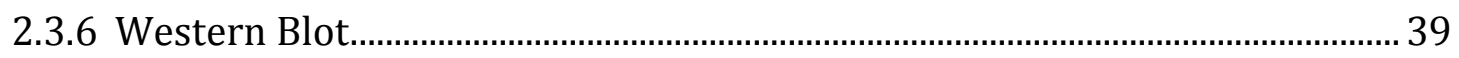

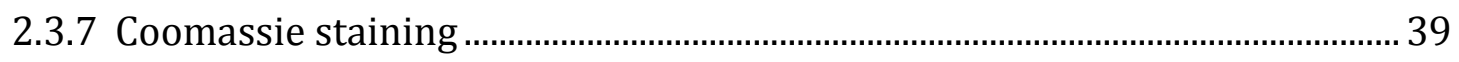

2.3.8 GST fusion protein purification for antibody generation ................................. 39

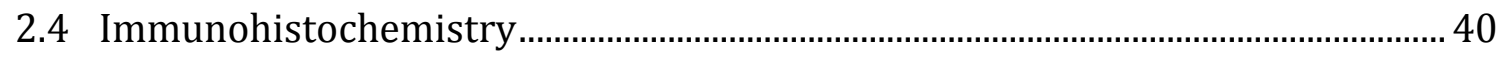

2.4.1 Fixation and immunofluorescent staining of embryos ..................................... 40

2.4.2 Fixation and immunofluorescent staining of larval tissues ............................ 40

2.4.3 Fixation and immunofluorescent staining of adult tissues ............................... 41

2.4.4 Fluorescent in situ hybridization (FISH) of embryos....................................... 41

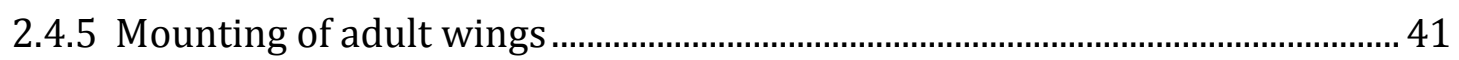

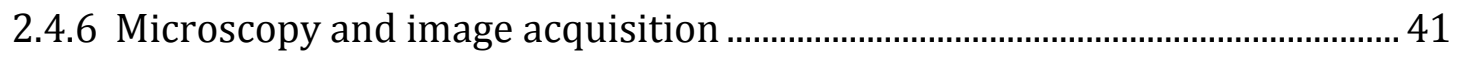

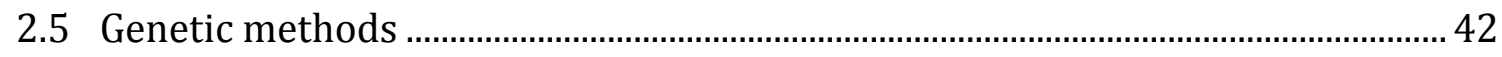

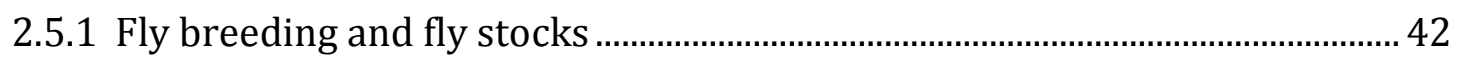

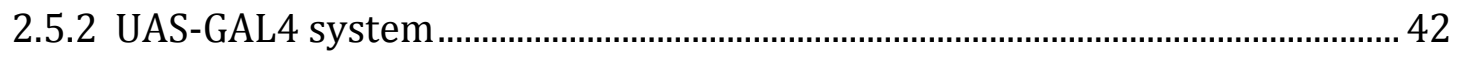

2.5.3 Generation of transgenic flies with white- background ................................... 42

2.5.4 Generation of transgenic flies using ФC31-mediated integration................... 43

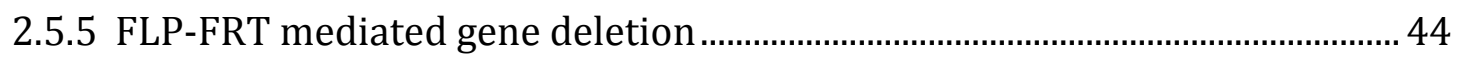

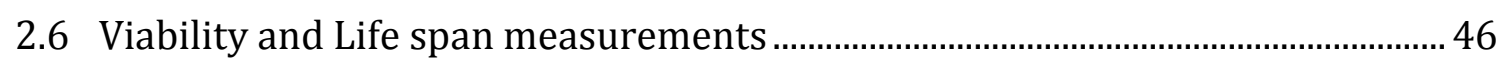

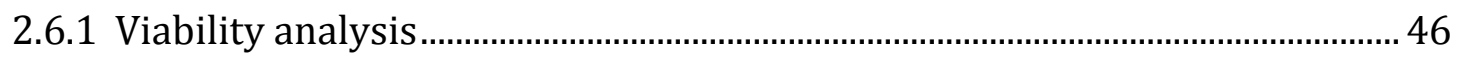

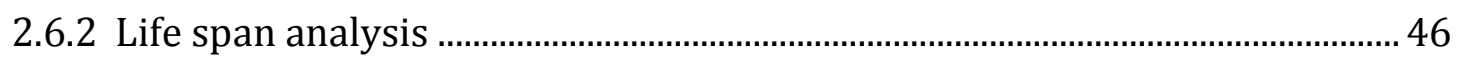

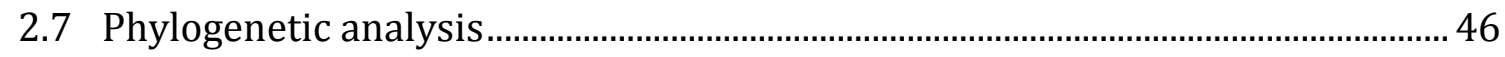

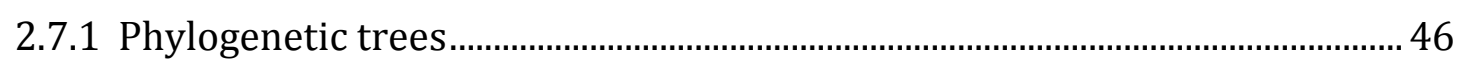

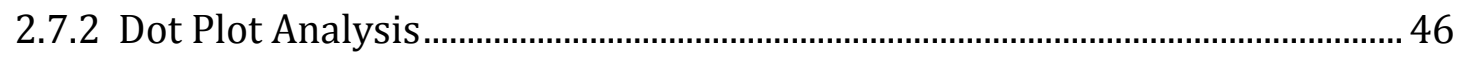

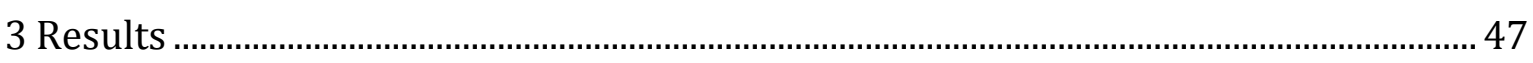

3.1 off track (otk) and the neighboring gene off-track2 (otk2, CG8964) are paralogs

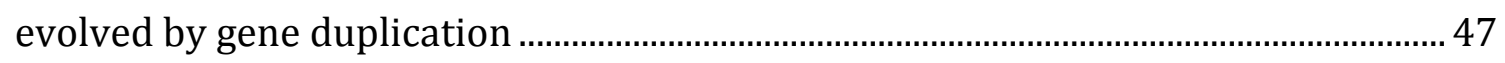

3.2 Biochemical interactions of Off-track and Off-track2 ........................................... 51

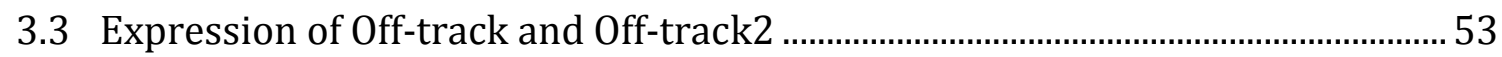


3.3.1 Expression of Off-track and Off-track2 reporter lines .53

3.3.2 mRNA Expression of Off-track and Off-track2 during embryonic development 56

3.3.3 Protein expression of Off-track and Off-track2 during embryonic development .59

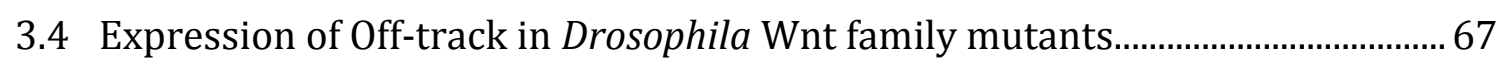

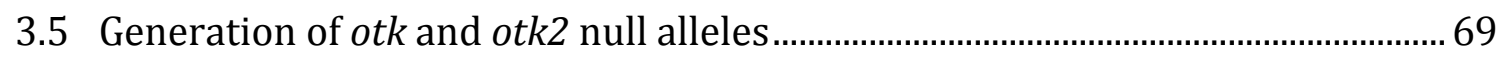

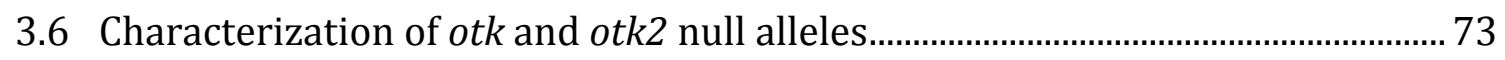

3.6.1 Novel otk and $o t k^{2}$ loss of function mutants are homozygous viable............. 73

3.6.2 Life span is not affected in novel otk and otk2 loss of function mutants ..... 74

3.6.3 Homozygous otk ${ }^{A 1}$ and otk,otk2 ${ }^{D 72}$ flies do not display any PCP or wing

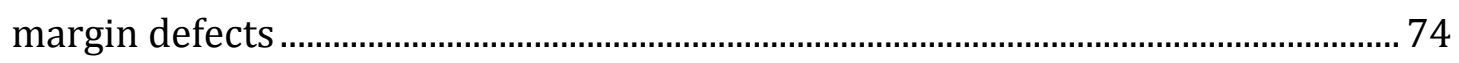

3.6.4 otk,otk2 ${ }^{D 72}$ homozygous mutants flies are male sterile..................................... 76

3.7 otk and otk2 interact with the Wingless receptor Frizzled .................................... 83

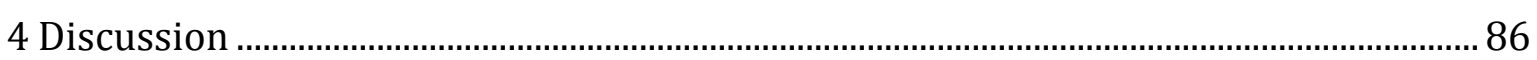

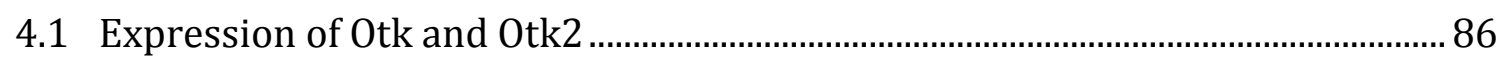

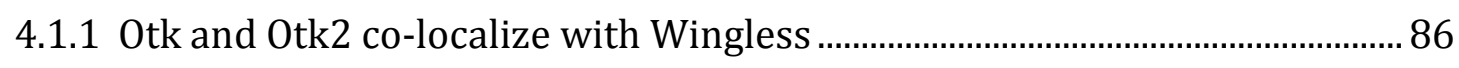

4.1.2 Otk and Otk2 co-localize with Derailed ............................................................. 87

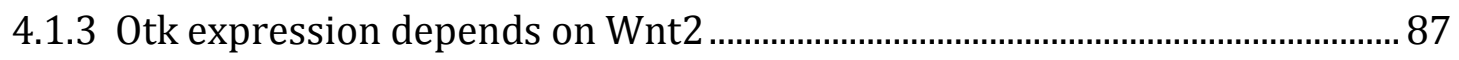

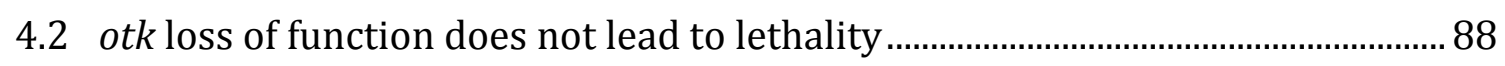

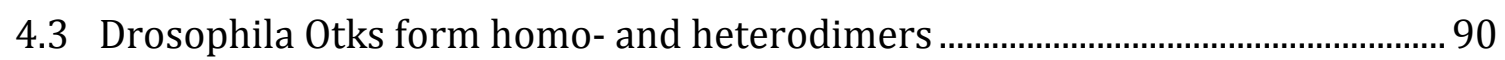

4.4 Otk and Otk2 might function as (co-)receptors in different Wnt signaling

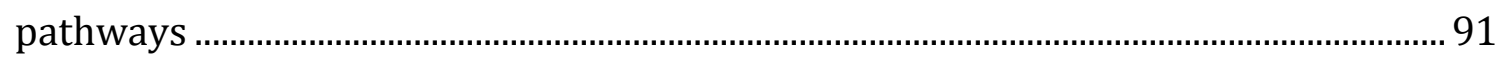

4.4.1 Possible role of Otk/Otk2 in Wnt2 signaling during male reproductive tract formation. 91

4.4.2 Possible role of Otk/Otk2 in Wnt5/Drl signaling ........................................... 93

4.4.3 Genetic interation of Otk/Otk2 with Fz ............................................................. 93

4.5 Possible molecular mechanisms of Wnt/Otk signaling .......................................... 94

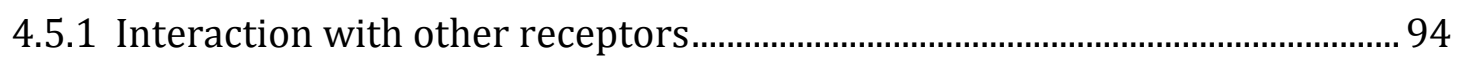

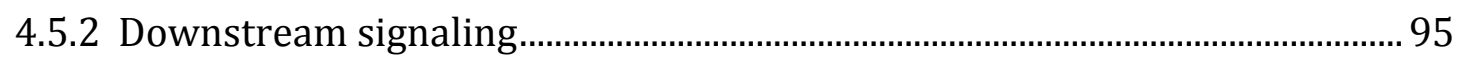

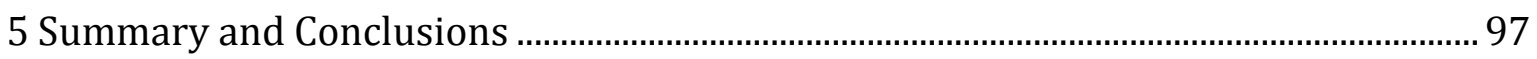

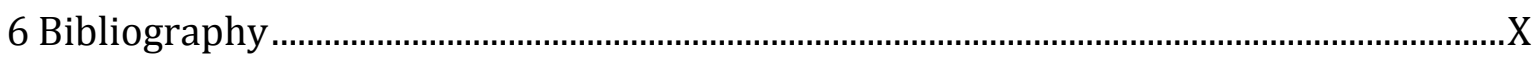

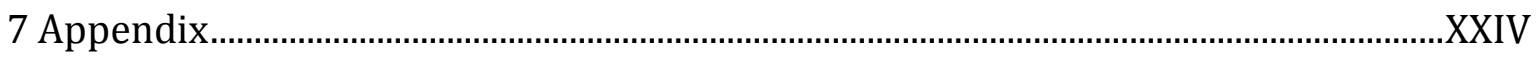




\section{ACKNOWLEDGMENTS}

It is a pleasure to express my gratitude to all those who supported me during the completion of this project.

First of all, I would like to thank Prof. Andreas Wodarz for giving me the exciting opportunity to join his department and for the supervision during these years. Thank you for the advice and support during the many turns of the project and for giving me the possibility to develop my own ideas and work independently.

Furthermore, I want to thank Prof. Annette Borchers and Prof. Reinhard Schuh for being members of my thesis committee and for helpful comments during thesis committee meetings. I also want to thank Gregor Bucher for discussion and help on phylogenetic analysis.

Huge thanks to all current and most of the former lab members of the Stem Cell Biology department for good times, much fun and making these years highly enjoyable! Thanks to the "Clonator" Mona as well as Katja and Claudia for the excellent technical support and keeping the lab running, Patricia for guidance through paperwork, Hamze for being a great friend and sharing the ups and downs of PhD life, Caro, Katja, Jaffer, Manu and Sascha for the fun times, my Bachelor and Master students Laura and Emma and the former lab members Ieva, Marilena, Gang, Nils and Tobi for many unforgettable moments.

I also would like to thank the members of the "Kafferunde" for having this tradition and for getting the day off to a good start.

I want to acknowledge the IMPRS for Molecular Biology for offering financial support in terms of a travel grant, which allowed me to visit an exciting EMBO meeting on Wnt signaling. In particular, I am grateful to Dr. Steffen Burkhardt, Kerstin Grüninger and Ivana Jurik for the enormous support of students and for the excellent organization of the program.

Many thanks to Birgit, Marie and Nils for proofreading and valuable comments.

During my study and PhD times in Göttingen many friendships arose and I feel very fortunate having such great people around me.

Finally, I am very grateful to my family for their truly unconditional support and to Nils and Paula for being at my side, great times together and letting life never become boring. 


\section{ABSTRACT}

Canonical and non-canonical Wnt signaling pathways have in common Frizzled (Fz) as the core Wnt receptor element. Recent findings implicate that not only the choice of Wnt ligands, but also the presence of additional components in the receptor complex determine signaling pathway specificity.

Vertebrate PTK7 (protein tyrosine kinase 7) encodes a catalytically inactive receptortyrosine kinase and is required for the control of planar cell polarity (PCP) in frogs and mice by acting as Fz co-receptor and inhibiting canonical Wnt signaling. Mutation of a Drosophila homolog of PTK7, the gene off-track (otk), was reported not to cause PCP phenotypes in the fly, but is suggested to block canonical Wingless signaling in embryonic patterning. We found that in contrast to previous reports, flies homozygous for a complete knock-out of otk are viable and fertile and indeed do not show PCP phenotypes. However, we discovered an otk paralog (otk2, CG8964). Otk and Otk2 are co-expressed throughout embryonic and larval development. They are highly expressed in the visceral mesoderm as well as in the nervous system and enriched at anterior commissures. Otk and Otk2 interact biochemically and possibly function redundantly in Wnt signal transduction.

Surprisingly, flies homozygous for a double knock-out of otk and otk2 are viable as well and neither show PCP nor Wingless signaling phenotypes. However, otk,otk2 double mutants are male sterile due to an obstruction in the ejaculatory duct and this is possibly linked to disturbed Wnt2 signaling. Overall defects in the nervous system cannot be observed, most likely because only a subset of neurons is affected. This could be explained by the abundance of receptors and co-receptors acting in a redundant manner. Indeed, expression data suggest a possible connection to Wnt5/Drl signaling. Furthermore, genetic and biochemical studies revealed that Otk/Otk2 genetically and biochemically interact with $f z 1$, indicating that Otk/Otk2 might also function as Fz1 co-receptors in the signal transduction of Wingless or other members of the Wnt family under certain circumstances.

These results suggests that Otk and Otk2 function as redundant receptors in several Drosophila Wnt signaling pathways, including Wnt2 as well as Wnt5/Drl signaling. 


\section{LIST OF FIGURES}

Fig. 1: Homeostatic range of Wnt signaling during development and disease.................1

Fig. 2: Canonical and non-canonical Wnt signaling pathways. .................................................

Fig. 3: Overview of canonical/ $\beta$-catenin dependent $W n t$ signaling . .....................................5

Fig. 4: PCP signaling regulates tissue organization in different systems. ...........................6

Fig. 5: Expression domains of segment polarity genes. ........................................................ 8

Fig. 6: Wnt5/Drl-mediated axon guidance during Drosophila embryonic CNS development.

Fig. 7: Modulation of Wnt signaling by many receptors....................................................... 12

Fig. 8: Non-Frizzled receptors for Wnt ligands. .............................................................. 13

Fig. 9: Structure of Ror receptor tyrosine kinases (RTKs) in different species.............. 15

Fig. 10: Model for PTK7 function in canonical and PCP signaling. ....................................... 19

Fig. 11: Scheme of ФС31 integrase-mediated trangene integration................................... 44

Fig. 12: Schematics of FLP-FRT recombination of two FRT containing transposons in trans.

Fig. 13: P-elements used for FLP-FRT recombination mediated deletion of otk and otk2.

Fig. 14: Alignment of the protein sequences of Otk and the gene product of CG8964.47 Fig. 15: Off-track (Otk) and Off-track2 (CG8964, Otk2) are paralogs evolved by gene duplication

Fig. 16: Structural similarity between Otk and Otk2. 50

Fig. 17: Alignment of the intracellular carboxy terminus of 0tk2 with the corresponding Otk sequence 50

Fig. 18: Off-track and Off-track2 form homodimers and heterodimers. 52

Fig. 19: Homo- and heterodimerization of Otk and Otk2 requires the transmembrane domain. .53

Fig. 20: Expression of Off-track and Off-track2 reporter lines.............................................. 55

Fig. 21: mRNA expression of Off-track in embryos. .57

Fig. 22: mRNA expression of Off-track2 in embryos......................................................... 58

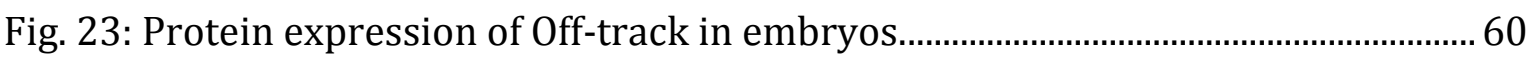

Fig. 24: Protein expression of Off-track2 in embryos

Fig. 25: Off-track is expressed in Wingless expressing neuroblasts and their neuronal progeny. 
Fig. 26: Off-track is highly expressed in the visceral mesoderm 63

Fig. 27: Off-track is expressed in three regions in the embryonic gut. 64

Fig. 28: Otk and Otk2 are expressed in the late embryonic nervous system and enriched at anterior commissures.

Fig. 29: Localization of Otk in embryos homozygous mutant for different Wnt family members 68

Fig. 30: Generation of otk and otk2 null alleles. 71

Fig. 31: Verification of $o t k$ and otk2 null alleles.

Fig. 32: Novel otk and otk2 loss of function mutants are homozygous viable and do not display any PCP or wing margin defects. 73

Fig. 33: Life span is not affected in novel otk and otk2 loss of function mutants 74 Fig. 34: Homozygous otk ${ }^{A 1}$ and otk,otk2D72 flies do not display any wing PCP or margin defects. 75

Fig. 35: Eyes of homozygous otk ${ }^{A 1}$ and $o t k$,otk2 ${ }^{D 72}$ flies do not display any PCP defects. 76

Fig. 36: Testes of otk,otk2 ${ }^{D 72}$ homozygous mutant males. 78

Fig. 37: Testes of otk,otk2 ${ }^{D 72}$ homozygous mutant males develop all stages of sperm development including motile sperm. 79

Fig. 38: Otk and Otk2 are expressed in both male and female genital discs. 80 Fig. 39: The male reproductive system of homozygous otk,otk2 mutants is surrounded by striated and smooth muscles.

Fig. 40: otk,otk2 loss of function leads to changes in the morphology of the ejaculatory duct.

Fig. 41: Ejaculatory duct obstruction in otk,otk2 homozygous mutant males 83

Fig. 42: Off-track and Off-track2 interact with Frizzled1 85

\section{LIST OF TABLES}

Table 1: Drosophila Wnt family members and their vertebrate homologs. .7

Table 2: Bacterial strains used in this study.

Table 3: Oligonucleotides used in this study 22

Table 4: Vectors used in this study. 26

Table 5: Constructs used in this study. 26 
Table 6: Primary antibodies used in this study................................................................... 28

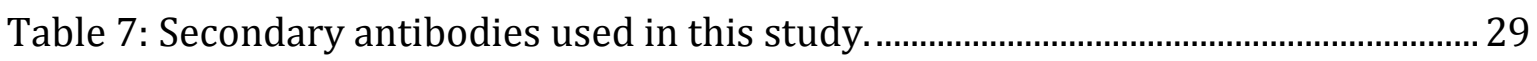

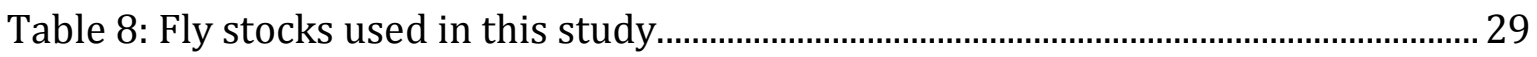

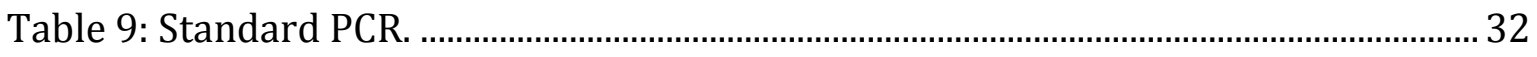

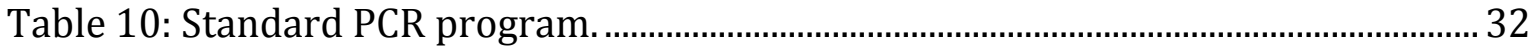

Table 11: PCR program for site-directed mutagenesis. ............................................................. 35

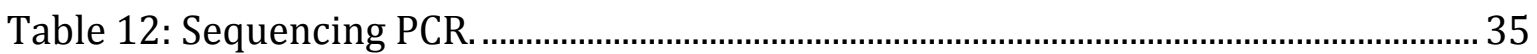

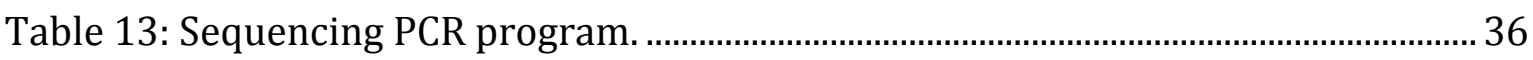

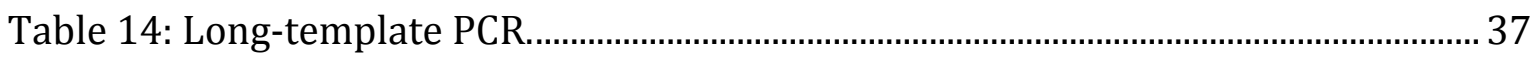

Table 15: PCR program for long-template PCR ……................................................................ 37

Table 16: otk,otk2 ${ }^{D 72}$ homozygous mutant males are male sterile...................................... 77

Table 17: Sterility otk,otk2 ${ }^{D 72}$ homozygous mutants males can be rescued by Otk

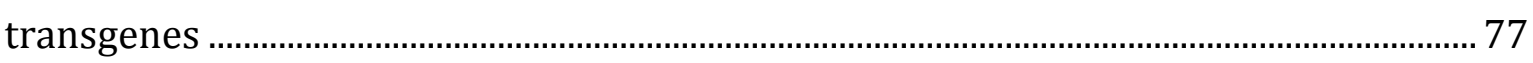

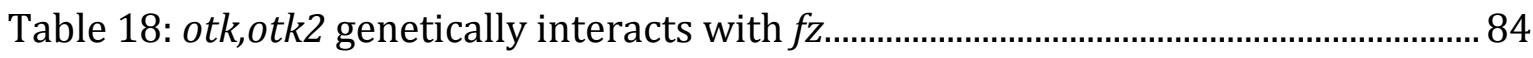




\section{INTRODUCTION}

\subsection{Wnt signaling}

Wnt signaling pathways are evolutionarily conserved and regulate many aspects of important biological phenomena, ranging from embryogenesis to cell behaviour and maintenance of adult tissue homeostasis.

During development Wnt signaling regulates processes such as cell fate determination, axis specification, gastrulation and neural development (Wodarz \& Nusse, 1998; Logan \& Nusse, 2004). In an adult organism, Wht signaling remains indispensable for the regulation of tissue maintenance, stem cell self-renewal as well as the regeneration of injured tissue (Logan \& Nusse, 2004; Reya \& Clevers, 2005; Clevers, 2006). The pathway is strictly kept in a homeostatic range, as exceeding signaling levels lead to diseases like cancer, whereas low levels of Wnt signaling could be one of the reasons for many degenerative conditions (Fig. 1) (Angers \& Moon, 2009).

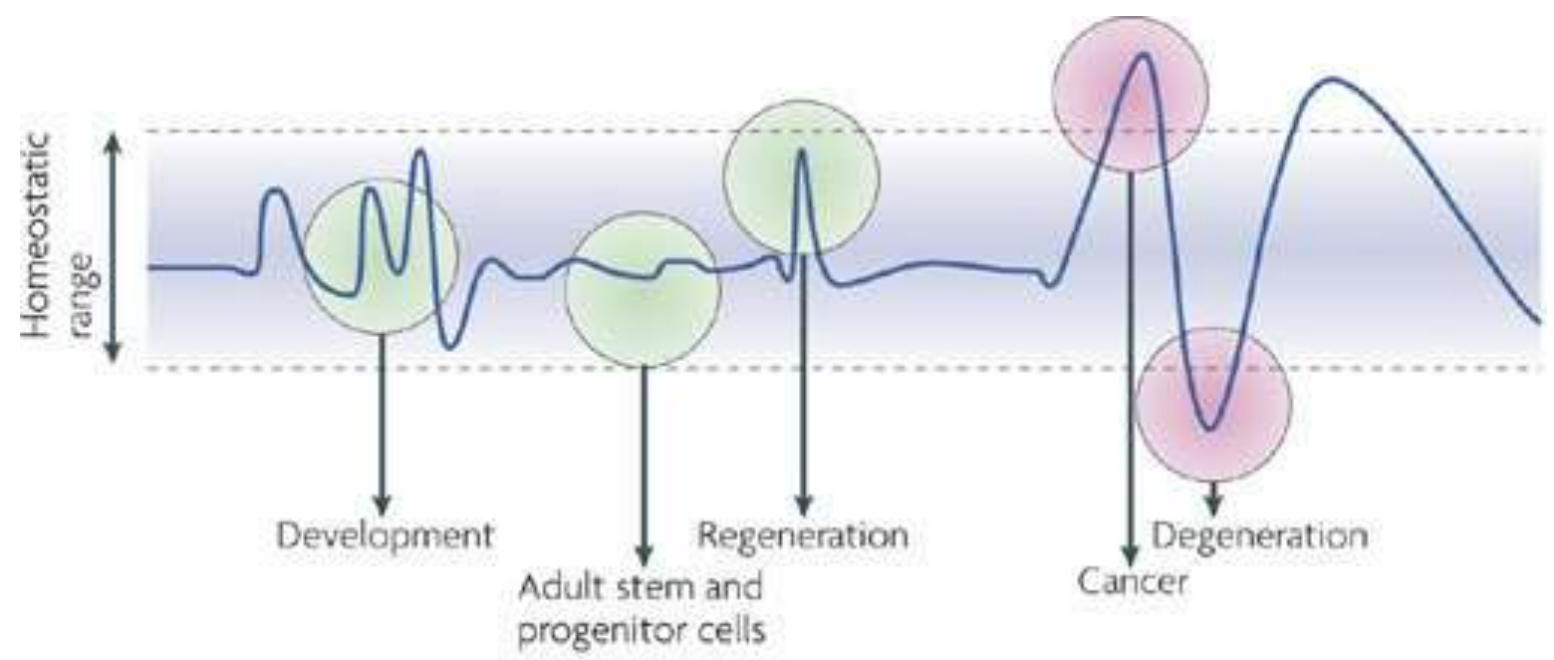

Fig. 1: Homeostatic range of Wnt signaling during development and disease.

Wnt signaling is dynamically regulated, but kept in a homeostatic range during development, stem cell maintenance and regeneration. Upregulated Wnt signaling leads to cancer and conversely low levels of Wnt signaling are one of the reasons for degenerative conditions. Taken from (Angers \& Moon, 2009).

Historically, the nomenclature "Wnt" was established after the Drosophila wingless $(w g)$ and the mouse int-1 oncogene, originally identified as the integration site for the mouse mammary tumor virus (Nusse \& Varmus, 1982), were shown to be homologous (Baker, 1987; Cabrera et al, 1987; Rijsewijk et al, 1987). This exciting 
discovery was one of the first examples of a gene implicated in cancer as well as in embryogenesis. Further studies in Xenopus revealed that ectopic expression of int1 induces duplication of the dorsal axis, suggesting a conserved role for this gene as an organizer and signal between germ layers that leads to pattern formation (McMahon \& Moon, 1989). The affiliation of wingless to the segment polarity group of genes paved the way for discovering the functional mechanism of int1/wingless by studying genetic and biochemical interactions with other genes in this group (NüssleinVolhard \& Wieschaus, 1980).

In the following years, the mammalian Wnt family, consisting of 19 members of secreted glycoproteins, became classified according to their ability to induce secondary dorsal-ventral axes in Xenopus embryos and to morphologically transform C57MG mouse mammary epithelial cells. For example, overexpression of Wnt1, Wnt3a and Wnt8 is sufficient to induce a double axis (Du et al, 1995) and transform C57MG cells (Wong et al, 1994; Olson \& Papkoff, 1994; Shimizu et al, 1997), whereas other Wnts such as Wnt4, Wnt5a and Wnt11 do not show this property. The ability of the above mentioned members of the Wnt family to transform mouse mammary epithelial cell lines and to induce a secondary axis in Xenopus embryos can be deduced from their ability to evoke an increase in the cytoplasmic levels of the protein $\beta$-catenin. This pathway is therefore referred to as the $\mathrm{Wnt} / \beta$-catenin or canonical pathway, because it was the first Wnt pathway identified.

On the other hand, for example Wnt5a and Wnt11 are required for correct convergent extension movements in Xenopus and zebrafish embryos, independent of $\beta$-catenin function (Heisenberg et al, 2000; Wallingford et al, 2001; Kilian et al, 2003; Veeman et al, 2003). Based on the grouping of Wnt family members into a canonical and a non-canonical class, the view of canonical ( $\beta$-catenin-dependent) pathways in contrast to non-canoncial ( $\beta$-catenin-independent) pathways emerged. Non-canonical pathways include the so far most characterized Wnt-calcium pathway and the planar cell polarity (PCP) pathway, which requires Wnt-c-Jun N-terminal kinase (JNK) activity (Fig. 2) (Montcouquiol et al, 2006). However, the degree to which these pathways overlap is presently unclear, but should not be underestimated.

Interestingly, the different Wnt pathways comprise both common components as well as pathway-specific players. The core components shared by all pathways mentioned are Wnt ligands, which act as morphogens, Fz transmembrane receptors and the cytoplasmic protein Dishevelled (Dsh/Dvl). Wht binding to Fz results in the activation 
of Dsh, which can be seen as the branching point of canonical and non-canonical pathways (Fig. 2) (Gao \& Chen, 2010). Canonical Wnt signaling controls cell proliferation and differentiation through influence on the transcription of $\beta$-catenin target genes, while PCP signaling regulates cell polarization and migration through regulation of the small GTPases Rho and Rac involved in remodeling of the actin cytoskeleton.

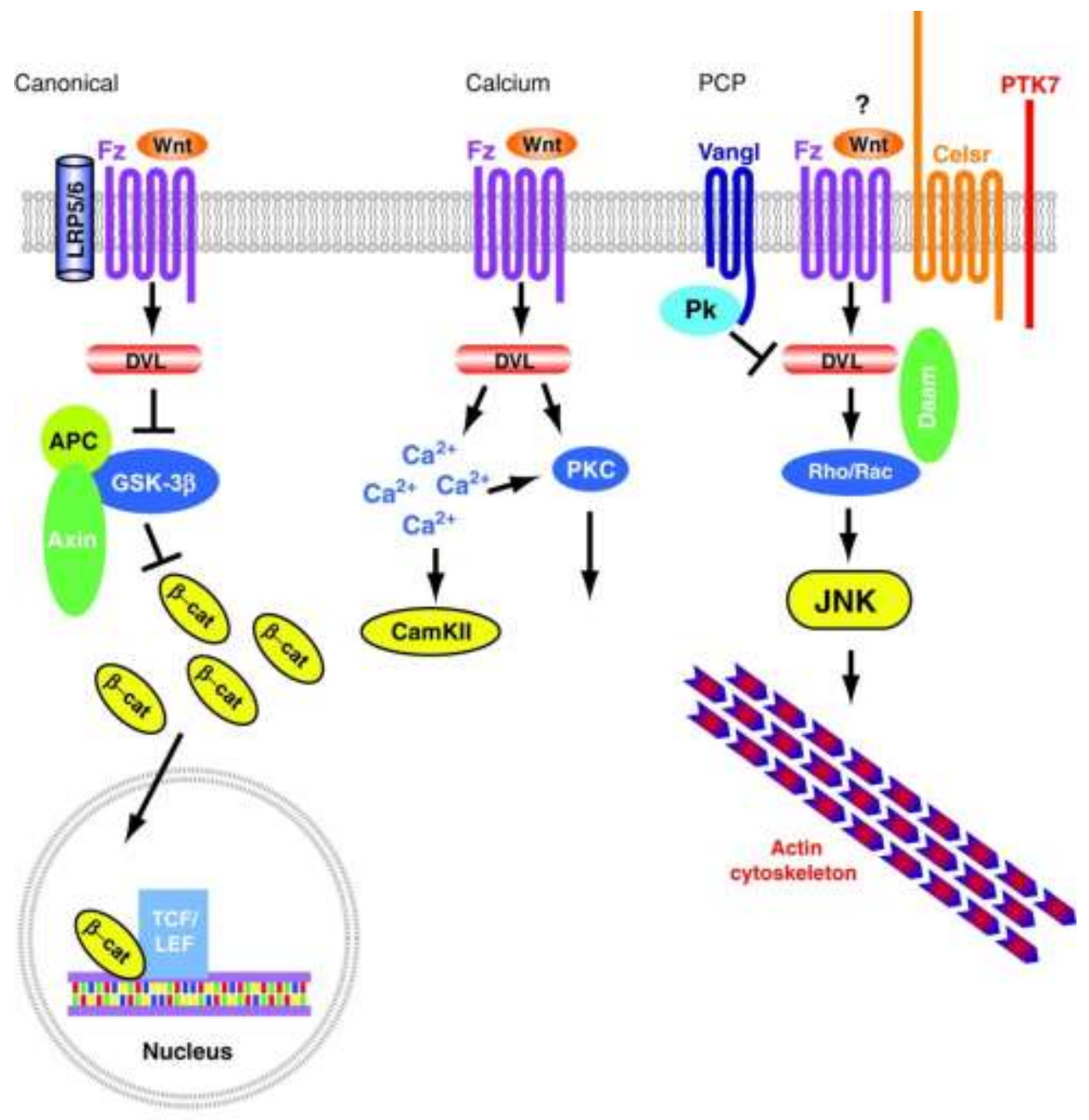

Fig. 2: Canonical and non-canonical Wnt signaling pathways.

In the canonical pathway, Wnt binding to $\mathrm{Fz}$ and its co-receptor LRP5/6 leads to activation of Dishevelled and this in turn inhibits $\beta$-catenin degradation. Increased cytoplasmic $\beta$-catenin can subsequently translocate to the nucleus and bind to members of the TCF/LEF family, activating transcription. An increase in the levels of intracellular calcium is the central event in the Wnt-Calcium pathway. This leads to the activation of Protein kinase C (PKC) as well as Calmodulin kinase II (CamKII) and each of these factors is then able to mediate intracellular responses. In the PCP pathway, Fz becomes activated, leading to Dvl activation. This then signals via the small GTPases Rho and Rac as well as C-Jun N-terminal kinase (JNK) to modulate the actin cytoskeleton. The PCP pathway also involves other transmembrane proteins like Vangl, Celsr and PTK7. Taken from (Montcouquiol et al, 2006), vertebrate nomenclature of the proteins is used. 


\subsection{Canonical / $\beta$-catenin-dependent signaling}

Mutations in the Drosophila genes dishevelled (dsh) and armadillo (arm, Drosophila homolog of $\beta$-catenin) showed similar cuticle defects as wingless mutant embryos (Perrimon \& Mahowald, 1987; Wieschaus \& Riggleman, 1987). In contrast, mutations in shaggy/zeste-white 3 (Drosophila homolog of Glycogen Synthase Kinase-3, GSK-3) cause the completely opposite phenotype (Siegfried et al, 1992). Epistasis studies uncovered that these genes were indeed core members of the same newly identified signaling pathway (Noordermeer et al, 1994). Consistently, the ability to induce a secondary axis in Xenopus embryos, as described for canonical Wnts, was also reported for Dsh, $\beta$-catenin and a dominant negative version of GSK-3 (Guger \& Gumbiner, 1995; He et al, 1995; Dominguez et al, 1995).

These combined observations from vertebrate and invertebrate model systems provided the first draft of the highly conserved canonical/ $\beta$-catenin-dependent signaling pathway. The critical event in this "classic" model of Wnt signaling is the stabilization and accumulation of cytoplasmic $\beta$-catenin, which can then translocate to the nucleus and activate transcription. In the absence of Wnt, the cytoplasmic levels of $\beta$-catenin are tightly regulated by a destruction complex consisting of amongst others Axin, Casein kinase $1 \alpha(\mathrm{CK} 1 \alpha)$, adenomatous polyposis coli (APC) and GSK-3 (Gordon \& Nusse, 2006). CK1 $\alpha$ and GSK-3 are responsible for $\beta$-catenin phosphorylation (Amit et al, 2002; Liu et al, 2002), which in turn results in ubiquitylation and subsequent proteasomal degradation (Aberle et al, 1997) (Fig. 3). Pathway activation occurs upon Wnt ligand binding to the seven passtransmembrane Frizzled (Fz) receptor (Yang-Snyder et al, 1996; Bhanot et al, 1996; Bhat, 1998) and its co-receptors LRP5 or -6 (Drosophila Arrow) (Pinson et al, 2000; Tamai et al, 2000; Wehrli et al, 2000). Wht binding to Fz triggers the phosphorylation of Dsh, which weakens the destruction complex (Yanagawa et al, 1995; Willert et al, 1997). Furthermore, GSK-3 and CK1 $\alpha$ phosphorylate LRP, which then recruits Axin to the plasma membrane, resulting in inactivation of the destruction complex (Davidson et al, 2005; Zeng et al, 2005). As a consequence, $\beta$-catenin is no longer degraded, accumulates in the cytoplasm and enters the nucleus, where it interacts with the DNA-binding proteins of the TCF/Lef family (T-cell factor and Lymphoid enhancer factor) (Behrens et al, 1996; Molenaar et al, 1996). $\beta$-catenin binding converts the TCF proteins into potent transactivators by displacing transcriptional co-repressors 
like Groucho (Cavallo et al, 1998; Roose et al, 1998) (Daniels \& Weis, 2005) and recruiting co-activators like Bcl9/Pygopus and Legless (Kramps et al, 2002; Hoffmans et al, 2005; Städeli \& Basler, 2005) (Fig. 3).
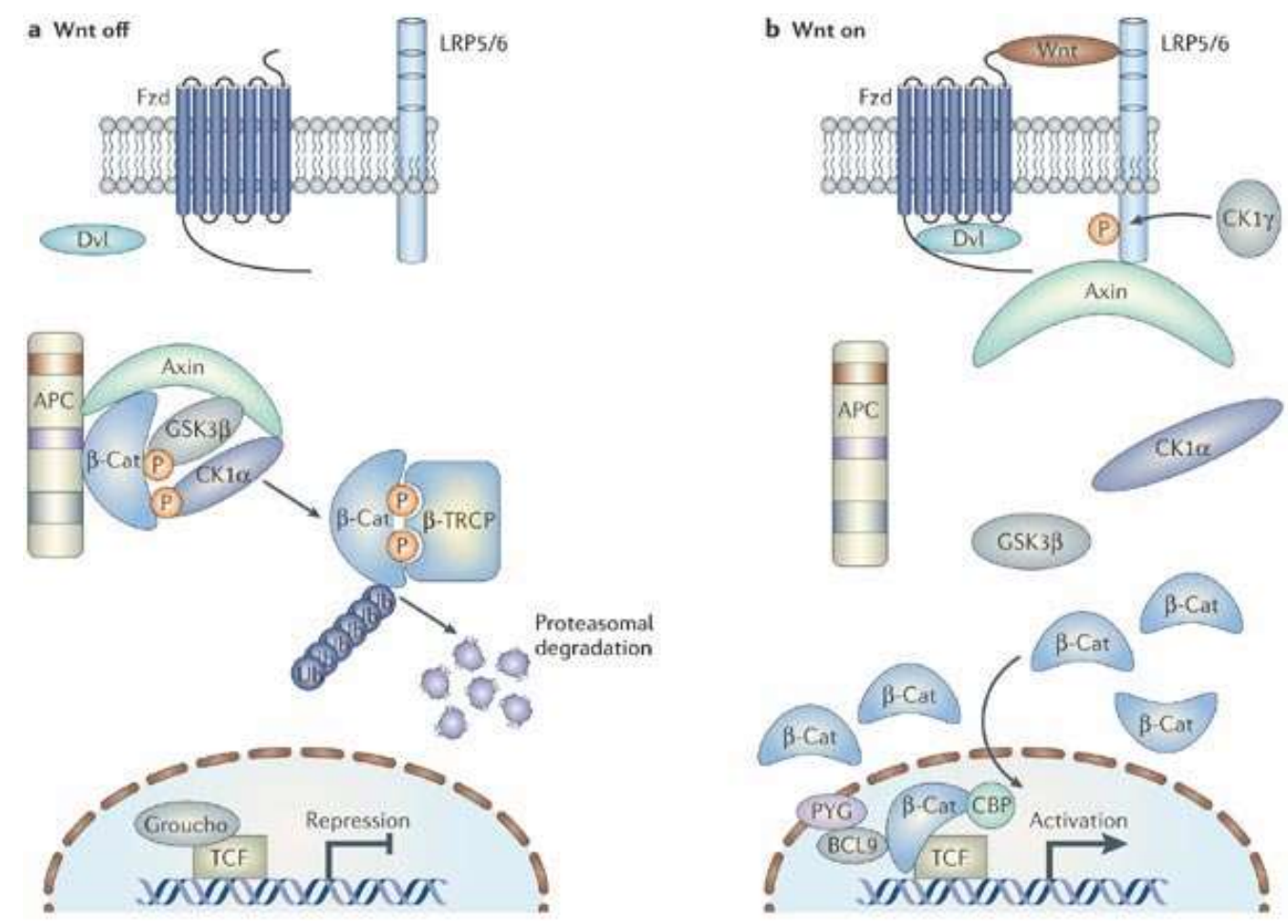

Fig. 3: Overview of canonical/ $\beta$-catenin dependent Wnt signaling.

(A) In the absence of Wnt ligands, $\beta$-catenin is held in a destruction complex by Axin and APC, phosphorylated by GSK3 $\beta$ and CK1 $\alpha$ and as a result degraded. Nuclear DNA binding proteins of the TCF/Lef family recruit transcriptional co-repressors like Groucho and thereby actively repress target genes. (B) Upon Wnt ligand binding, Fz binds to Dvl and LRP5/6 is phosphorylated by CK1 $\alpha$. This recruits Axin to the membrane and the destruction complex disassembles. $\beta$-catenin accumulates in the cytoplasm and translocates to the nucleus. It displaces the transcriptional co-repressors and instead recruits activators proteins like Bcl9/Pygopus and Legless, leading to the activation of TCF target genes. Taken from (Barker \& Clevers, 2006)

\subsection{Non-canonical / PCP signaling}

The best characterized non-canonical Wnt pathway is the so-called planar cell polarity (PCP) pathway. It was initially described in Drosophila, where it is responsible for establishing polarity in the plane of an epithelium perpendicular to the apico-basal axis of polarity. Flies mutant for PCP genes have typical phenotypes like the disturbed orientation of wing hairs and bristles on thorax and legs, as well as the disturbed orientation of ommatidia in the eyes. In vertebrates, PCP signaling 
controls convergent extension movements, tissue organization and collective cell movement (Zallen, 2007).
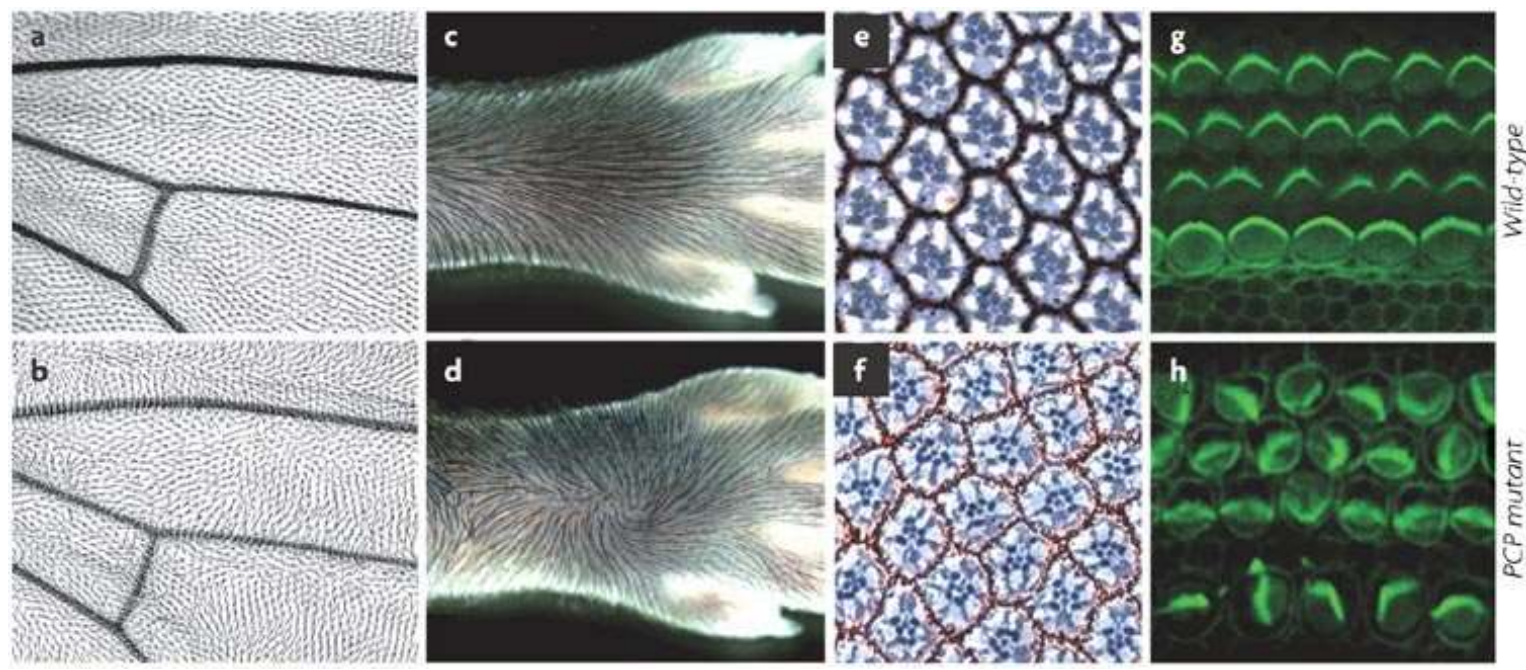

Fig. 4: PCP signaling regulates tissue organization in different systems.

(A) Drosophila wings are covered with hairs which all point distally. (B) Mutations in PCP genes like frizzled lead to a disruption of this organization, creating swirls and waves on the wing. (C,D), Similarly, the pattern of mammalian fur is disturbed in PCP mutants (D). (E,F) The ommatidia in the Drosophila eye each contain eight photoreceptors, arranged in a characteristic pattern, which becomes disorganized in PCP mutants. (G) Stereocilia in the sensory hair cells of the mammalian cochlea are polarized, this polarity is disturbed in PCP mutants (H). Taken from (Seifert \& Mlodzik, 2007).

Frizzled and Dsh are key players in this pathway and mutants display clear PCP phenotypes. The pathway diverges downstream of Dsh in that it does not involve Axin, GSK-3 or $\beta$-catenin, which are core components of canonical signaling.

Dsh contains three conserved domains - a DIX, PDZ and DEP domain and the latter one was described to be required for Drosophila and vertebrate PCP signaling by associating with the small GTPase Rac, which then activates JNK (Boutros et al, 1998; Axelrod et al, 1998; Heisenberg et al, 2000; Wallingford \& Habas, 2005). Other components that were shown to act both in Drosophila and vertebrate PCP signaling include Strabismus/Van Gogh-like, Prickle, and Flamingo/Celsr (Veeman et al, 2003). Strikingly, in Drosophila no Wnt protein could be shown to actively participate in PCP signaling. This is in contrast to the situation in vertebrates, where both Wnt5a and Wnt11 have been implicated to be essential for correct convergent extension in mouse (Qian et al, 2007) and Zebrafish (Heisenberg et al, 2000). 


\subsection{Drosophila Wnt family}

Wnt proteins are a family of secreted glycoproteins that play pivotal roles in development and disease (Logan \& Nusse, 2004). While the mammalian genome encodes 19 Wnt proteins, Drosophila has seven Wht genes (Table 1), but only one member of the family, Wingless, is well characterized. None of the other Wnt family members was discovered in screens for mutant phenotypes, but rather by their molecular homology. Furthermore it should be mentioned that no Wnt has so far been shown to be required for PCP signaling in Drosophila.

Table 1: Drosophila Wnt family members and their vertebrate homologs.

\begin{tabular}{cc}
\hline Drosophila & Vertebrates \\
\hline Wingless & Wnt1 \\
Wnt2 & Wnt7 \\
Wnt3/5 & Wnt5 \\
Wnt4 & Wnt9 \\
Wnt6 & Wnt6 \\
Wnt8 & - \\
Wnt10 & Wnt10 \\
\hline
\end{tabular}

\subsubsection{Wingless $(\mathrm{Wg})$}

As already mentioned, the segment polarity gene wingless $(\mathrm{wg})$ is the Drosophila homolog of the mouse int1-gene (Rijsewijk, 1987; Cabrera 1987; Baker, 1987). The term "segment polarity gene" was coined for one class of mutants retrieved in a genetic screen that shared a similar patterning phenotype during embryogenesis (Nüsslein-Volhard \& Wieschaus, 1980). Another mutant allele of the wingless gene leading to the loss of wing tissue had been identified earlier (Sharma \& Chopra, 1976).

The segmental subdivisions of the Drosophila embryo are controlled by the temporal expression of the maternal, gap and pair rule classes of genes. Genes of the segment polarity class, which includes wingless, are required in each segment for correct pattern formation (for review, see (Ingham, 1988)). Mutations in wg and other segment polarity genes cause the posterior portion of each segment to be deleted and replaced by a mirror-image duplication of the anterior portion. As a consequence, the segmentation of the embryonic epidermis, which is normally covered with naked 
cuticle and denticle belts in an alternating way, is severely disturbed in $w g$ mutant embryos, leading to a complete lawn of denticle (Nüsslein-Volhard \& Wieschaus, 1980).

During germ band extension of early Drosophila embryonic development, $\mathrm{wg}$ is expressed as a continuous stripe in the posterior region of each of the fourteen parasegments (Baker, 1987; van den Heuvel et al, 1989), next to the expression domain of the segment polarity gene engrailed (en), which comprises the anterior part of each parasegment (DiNardo et al, 1985). $w g$ is required for formation of both the segment boundary and the parasegment groove, which forms at the junction of $w g$ - and en-expressing cells (Perrimon \& Mahowald, 1987) (Fig. 5).

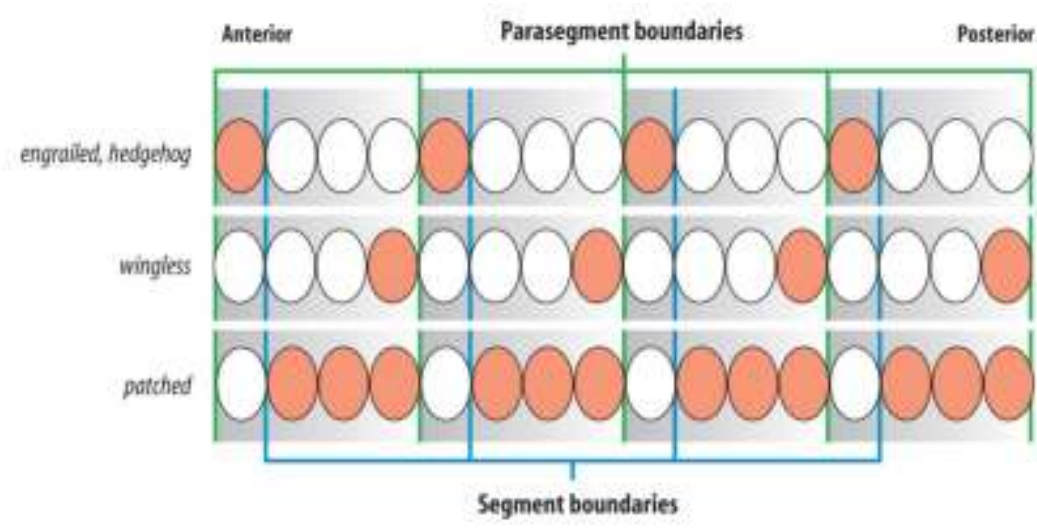

Fig. 5: Expression domains of segment polarity genes.

During the cellular blastoderm stage, wingless starts to be expressed at the posterior margin of each parasegment, whereas engrailed is expressed at the anterior margin together with hedgehog. The segment boundaries lie posteriorly to the engrailed expression domain. Taken from (Wolpert et al, 2007).

During embryonic development, $w g$ is necessary for multiple phases of patterning of the segmented trunk ectoderm (Bejsovec \& Martinez Arias, 1991; Dougan \& DiNardo, 1992; Bejsovec \& Wieschaus, 1993) as well as for proper development of the head (Schmidt-Ott \& Technau, 1992). Subsets of neuroblasts in each segment of the early central nervous system require non-autonomous $w g$ activity for determination and subsequent delamination (Chu-LaGraff \& Doe, 1993). Furthermore, wg is needed for patterning of the developing midgut (Immerglück et al, 1990; Thüringer \& Bienz, 1993; Bienz, 1994) as well as for growth of the malphigian tubules (Skaer \& Martinez Arias, 1992). These studies revealed that $\mathrm{Wg}$ is a protein secreted both within and across tissues and affects patterning also in neighboring cells (Klingensmith \& Nusse, 1994). Analysis of the central nervous system of $w g$ null mutant embryos has revealed only subtle defects, indicating only a minor role in neurogenesis during late 
embryonic development (Patel et al, 1989). After embryogenesis, wg is involved in patterning of imaginal discs as well as several other adult structures (reviewed in Klingensmith \& Nusse, 1994).

\subsubsection{Wnt2}

Wht2 was discovered as a member of the Drosophila Wnt family of genes (Russell et $a l, 1992)$ and is required for the development of the male reproductive tract (Kozopas et al, 1998). Drosophila testes are surrounded by a sheath consisting of outer malespecific pigment cells and an inner muscle layer (Kozopas et al, 1998; Susic-Jung et al, 2012). Wnt2 mutant flies are viable, but male-sterile due to both the absence of pigment cells and defects in development of the inner muscle layer of the testis sheath. Furthermore, ectopic expression of Wnt2 in females results in the formation of normally male-specific pigment cells, indicating that Wnt2 controls sexually dimorphic development (Kozopas et al, 1998). This was supported by another study showing that male-specific embryonic gonadal Wnt2 expression is necessary and sufficient to induce the formation of pigment cells (DeFalco et al, 2008a).

Further analyses revealed that Wnt2 is also required for the correct attachment of direct flight muscles to their epithelial sites, leading to flightlessness in homozygous mutant animals (Kozopas \& Nusse, 2002).

\subsubsection{Wnt4}

The Wnt4 gene maps close to wingless ( $\mathrm{wg}$ ) (30 kb upstream) and was isolated from a library of genomic fragments that associate in vivo with Ultrabithorax proteins. Wnt4 is expressed in a segment polarity-like pattern and in the visceral mesoderm, thereby partially overlapping with Wingless expression (Graba et al, 1995). Their coexpression was ascribed to the sharing of cis-regulatory elements (Gieseler et al, 1995). Functionally, Wnt4 antagonizes Wingless signaling both in the Drosophila ventral epidermis as well as in a heterologous system, the Xenopus embryo, most likely upstream of the activation of transcriptional targets (Gieseler et al, 1999). In contrast to the ventral epidermis, $w g$ and Wnt4 are not co-expressed in the dorsal epidermis and produce distinct responses in these cells. Moreover, Wnt4 requires Hedgehog to elicit its effects, whereas wg acts independently of hedgehog (Buratovich et al, 2000). 
In contrast to Drosophila embryogenesis, when $w g$ and Wnt4 can have either antagonistic or distinct functions, they induce similar cellular responses during imaginal development in that overexpression of Wnt4 or Wg leads to notum-to-wing transformation and alters the morphogenesis of adult appendages (Gieseler et al, 2001).

\subsubsection{Wnt5 (DWnt3)}

The Wnt5 gene, which has also been described as $D W n t 3$, encodes an unusual Wnt protein that contains a long amino terminal extension without any known conserved domains. Its molecular mass of $112 \mathrm{kDa}$ is almost three times larger than the one of the other Wnt family members (Eisenberg et al, 1992; Russell et al, 1992). Wnt5 protein localizes to the CNS during late embryogenesis and overexpression leads to malformation of commissures from stage 13 on, most likely due to a reduction of axon bundles crossing the midline (Fradkin et al, 1995). Wnt5 was identified as a ligand for Derailed (Drl), the Drosophila Ryk homolog, in a screen for mutations that suppress the ability of Drl to switch axons to the anterior commissure (AC) when misexpressed by neurons in the posterior commissure (PC) (Yoshikawa et al, 2003). Wnt5 mutant flies are homozygous viable and fertile, but uncoordinated. When Drl was misexpressed in a Wht5 mutant background, its PC-to-AC switching activity was strongly suppressed, suggesting that Drl requires Wnt5 to switch axons (Fig. 6) (see 1.5.4). High levels of Wnt5 mRNA were found in the posterior commissures, whereas the protein was enriched on both commissures compared to the longitudinal tracts. Based on these findings Wnt5, was proposed to act as a repulsive ligand for Drlexpressing axons that cross along the AC (Yoshikawa et al, 2003). Another study discovered that Wnt5 is required for the separation and defasciculation of early axonal projections that will form the mature commissural and longitudinal connectives. Furthermore, in Wnt5 mutant embryos, no separation of midlinecrossing axons into two distinct commissures occurs and consequently no axons cross along the AC (Fradkin et al, 2004). 


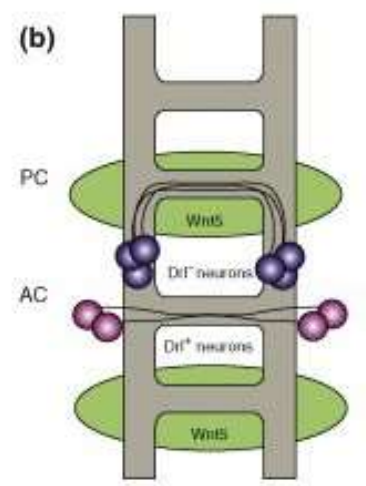

Wildtype
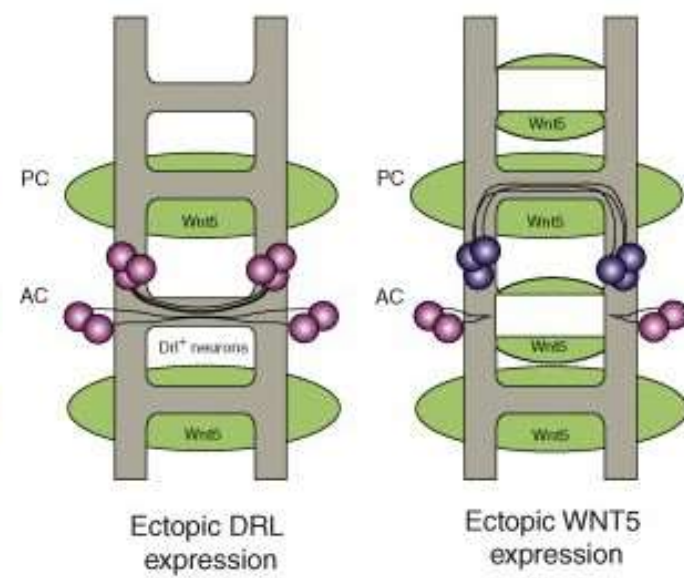

Fig. 6: Wnt5/Drl-mediated axon guidance during Drosophila embryonic CNS development.

Wnt5 is expressed in the posterior commissure (PC) and repels Drl-expressing axon, which therefore cross the midline across the anterior commissure (AC). Upon ectopic expression of Drl in PC neurons, these also cross along the AC. Ectopic Wnt5 expression along the midline leads to a loss of the AC. Depicted are the axons of eagle-expressing neuronal lineage. Taken from (Fradkin et al, 2010).

In contrast to its role in embryonic axon guidance, genetic analyses of Wnt5/Drl signaling in two Drosophila postembryonic brain centers, the mushroom body (MB) and the antennal lobes (ALs), suggest that Drl can also act to sequester Wnt5 to prevent it from interacting with other receptors (Moreau-Fauvarque et al, 1998; Grillenzoni et al, 2007; Yao et al, 2007; Sakurai et al, 2009). In Drosophila, odor information received by olfactory receptor neurons, located in the antennal and maxillary palps, is processed by glomeruli organized in the antennal lobe to form synapses with projection neurons which then relay olfactory information to the mushroom body calyx and the lateral horn. In $d r l$ mutants, the MB dorsal lobes are lost and the medial lobes are fused across the midline (Moreau-Fauvarque et al, 1998; Grillenzoni et al, 2007). Overexpression of $\mathrm{Drl}$ phenocopies the Wnt5 mutant phenotype and overexpression of Wnt5 leads to a $d r l$ mutant-like phenotype, suggesting that Wnt5 and $d r l$ act antagonistically during MB development (Grillenzoni et al, 2007). Two studies revealed that Drl plays a similar Wnt5 sequestration function during AL development (Yao et al, 2007; Sakurai et al, 2009).

\subsubsection{Other Wnt family members}

Wnt6 and Wnt10 are located on the second chromosome within $100 \mathrm{~kb}$ to Wnt4 and wingless $(w g)$, however due to a lack of loss of function data, little is known about their functions. The expression of Wnt6 is weak in embryos, but identical to that of $w g$ in imaginal discs, suggesting that either both genes are under the control of the same 
enhancer or Wnt6 is induced by earlier Wg expression (Janson et al, 2001). Wnt10 is expressed in the embryonic mesoderm, central nervous system and gut (Janson et al, 2001).

Another Wnt family member, WntD (Wnt inhibitor of Dorsal; Wnt8 in Llimargas \& Lawrence, 2001), acts as a feedback inhibitor of the Drosophila NF- $\kappa \mathrm{B}$ homolog Dorsal during both embryonic patterning and the Toll-mediated innate immune response (Gordon et al, 2005; Ganguly et al, 2005; Gordon et al, 2008).

\subsection{Wnt co-receptors}

The identification of Frizzled transmembrane proteins as Wnt receptors in Drosophila cell culture (Bhanot et al, 1996) provided the essential link between extracellular Wnts and the intracellular components of Wnt signal transduction. However, in the past decade, other transmembrane receptors have been shown to bind Wnt ligands, resulting in the activation of diverse intracellular signaling pathways. Apart from members of the Frizzled receptor family, Wnt receptors include the low-density lipoprotein receptor-related protein (LRP, Drosophila Arrow), the protein tyrosine kinase 7 (PTK7) as well as the receptor tyrosine kinase-like orphan receptor (Ror) and the related to tyrosine kinase (Ryk) families. Wnt interactions with these receptors often lead to cellular effects that are unrelated to $\beta$-catenin, possibly mediating a variety of non-canonical signaling pathways (Van Amerongen et al, 2008) (Fig. 7).

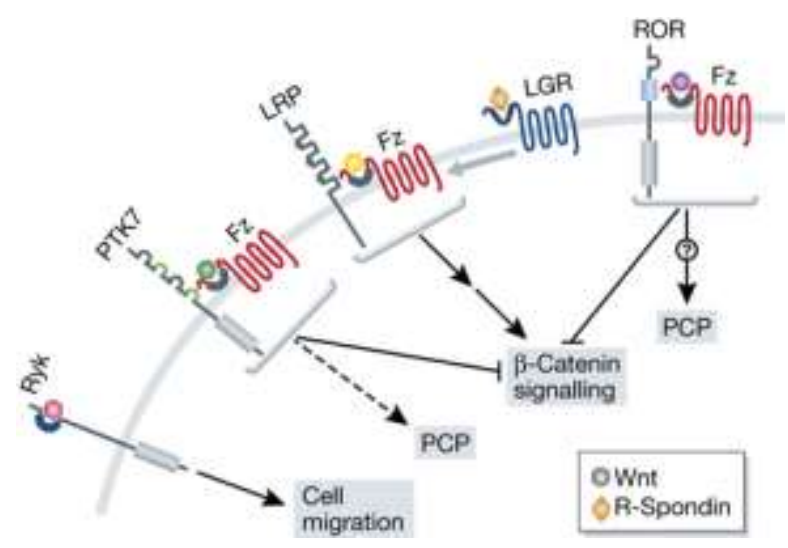

Fig. 7: Modulation of Wnt signaling by many receptors.

Frizzled transmembrane proteins are considered to be the main Wnt receptors; however, the final result of Wnt-Frizzled interaction is influenced by co-receptors. Wnt/Fz/LRP signaling activates canonical signaling, whereas PTK7 and Ror have been shown to inhibit $\beta$-catenin-dependent signaling. Ryk can function independently of Fz. Taken from (Vincent \& Beckett, 2011). 


\subsubsection{Modulation of Wnt signaling outcome by (co-)receptors}

Apart from the originally identified Frizzled receptors, other transmembrane Wnt receptors have been identified and subsequently shown to be involved in modulating the signaling outcome (Fig. 8). For example, Wnt5a and Wnt11, typically known as regulators of non-canoncial Wht signaling, can also induce canonical signaling depending on receptor context (He et al, 1997; Tao et al, 2005; Mikels \& Nusse, 2006; Cha et al, 2008). Thus, receptors can be bound by different Wnts and these in turn can activate multiple receptors. This suggests that Wnt signaling outcomes are dictated largely by the nature of the Wnt ligand as well as the nature of the Wnt receptor expressed in a tissue (Mikels \& Nusse, 2006; van Amerongen et al, 2008; van Amerongen \& Nusse, 2009; Vincent \& Beckett, 2011).

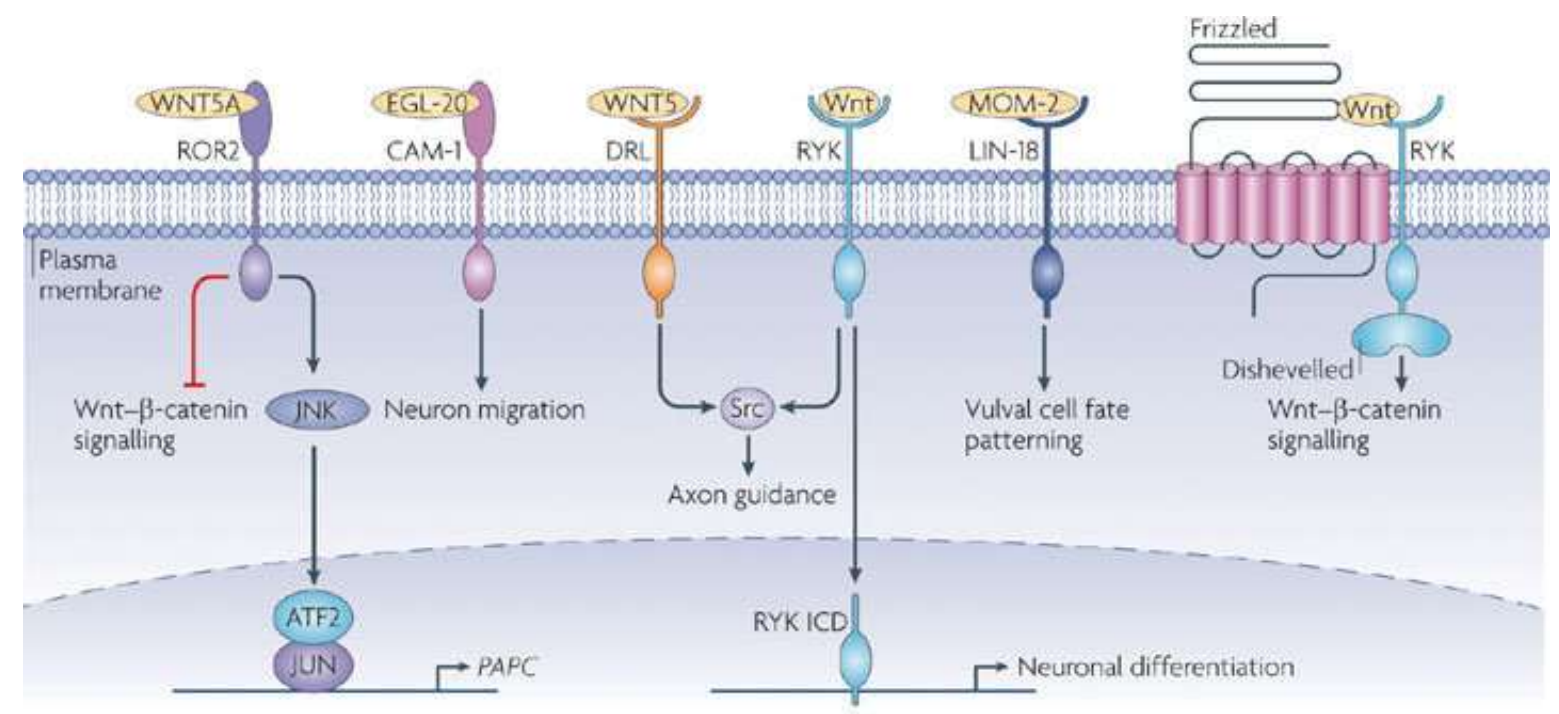

Fig. 8: Non-Frizzled receptors for Wnt ligands.

Wnt signals can be transduced by other transmembrane receptors apart from the Frizzled proteins. This signal transduction might regulate Wnt signaling positively or negatively and thereby modulate the biological outcome. Vertebrate Ror- 2 and its C. elegans homolog Cam-1 interact with Wnt5 or Egl20, respectively via their CRD domains. This activates JNK signaling in vertebrates or neuronal migration in C. elegans. Vertebrate Ryk, Drosophila Drl and C. elegans Lin-18 interact with their respective Wht ligands via WIF domains. Drl and Ryk control axon guidance via recruitment of Src family kinases. In vertebrates, the cleaved intracellular domain (ICD) of Ryk can translocate to the nucleus and regulate neuronal differentiation. Additionally, Ryk can form a complex with Fz and Dsh and this might be important for $\beta$-catenin-dependent signaling. Taken from (Angers \& Moon, 2009). 


\subsubsection{LRP / Arrow}

Members of the LRP (low density lipoprotein receptor-related protein; LDL-related protein) family are highly important Frizzled co-receptors for canonical signaling. For example, Arrow, the Drosophila homolog of LRP, is essential for Wingless signal transduction (Wehrli et al, 2000). Similarly, LRP6 was shown to function as a coreceptor for Wnt signal transduction in Xenopus (Tamai et al, 2000) and mice (Pinson et al, 2000). So far, no modulation of PCP signaling was observed for Arrow/LRP. Therefore, this class of co-receptors is believed to act strictly canonical (Vincent \& Beckett, 2011).

\subsubsection{Ror}

Ror (Receptor tyrosine kinase-like orphan receptor) proteins belong to the Receptor tyrosine kinase family (RTK), a superfamily of transmembrane glycoproteins involved in many critical processes like proliferation and differentiation as well as cell migration (Lemmon \& Schlessinger, 2010). Ror-family RTKs are evolutionarily conserved in invertebrates and vertebrates and the members in vertebrates are Ror1 and Ror2 (Minami et al, 2010). Structural characteristics of vertebrate Ror proteins are their extracellular Frizzled-like cysteine-rich domain (CRD), which binds to Wnt ligands, and their Kringle domain, which is membrane-proximal. In addition to the CRD and Kringle domain, most vertebrate Ror-family members possess extracellular immunoglobulin (Ig)-like domains (Minami et al, 2010). The intracellular region of Ror contains a conserved tyrosine kinase domain, as well as a proline-rich (PRD) and two serine/threonine-rich domains (S/TRD1 and S/TRD2) (Fig. 9). The functions of the different protein domains are diverse and extensively reviewed in (Forrester, 2002; Yoda et al, 2003; Minami et al, 2010). Drosophila has two Ror family members and they neither possess extracellular Ig-like domains nor intracellular S/TRD and PRD domains (Fig. 9). Drosophila Ror is the homolog of vertebrate Ror1 and its closest ortholog in the Drosophila genome is Dnrk (Drosophila Neurospecific receptor kinase), which is most similar to vertebrate MuSK (Muscle-specific kinase) (Green et al, 2008). 


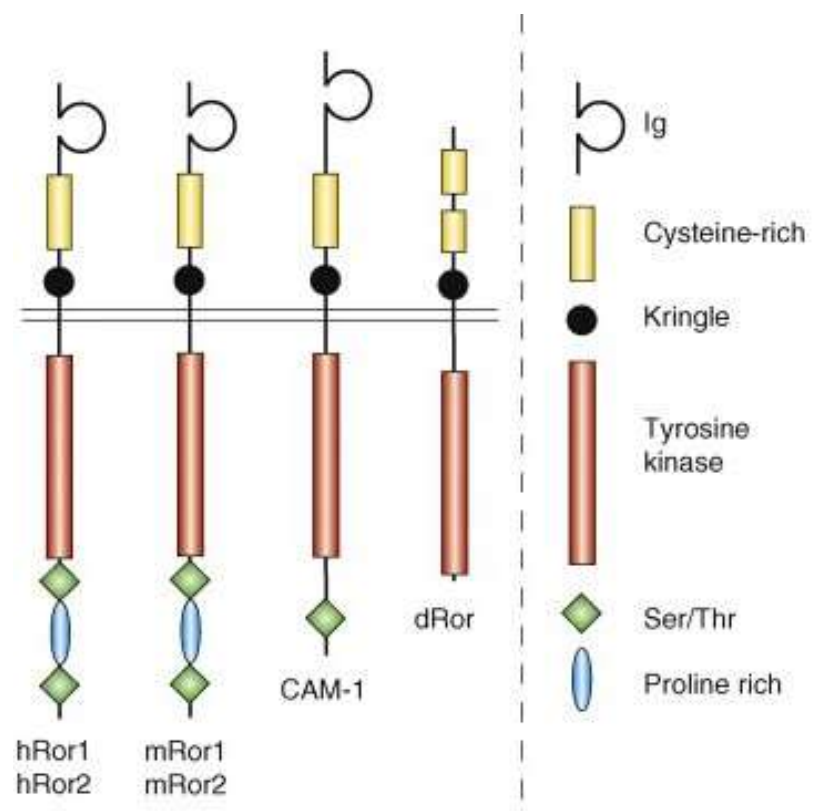

Fig. 9: Structure of Ror receptor tyrosine kinases (RTKs) in different species.

The extracellular region of human (hRor1 hRor2), mouse (mRor1, mRor2), C. elegans (CAM-1) and Drosophila (dRor) contains a cystein-rich as well as a Kringle domain. The cytoplasmic region is characterized by a tyrosine kinase domain, two serine/threonine-rich domains and a proline-rich domain. In contrast to its homologs, dRor does neither have extracellular Ig domains nor intracellular serine/threonine-rich and proline-rich domains. Taken from (Green et al, 2008).

The function of Ror proteins in development has been extensively studied in different organisms. Disruptions of human Ror2 leads to skeletal defects like brachydactyly (Oldridge et al, 2000; Schwabe et al, 2000) and dwarfism (Bokhoven et al, 2000; Afzal et al, 2000). Similar phenotypes were observed in mice mutant for Ror2 and the phenotype is enhanced in Ror1,Ror2 double knock-outs (Ho et al, 2012). Xenopus Ror2 (Xror2) transcripts were detected in gastrula and neurula embryos (Hikasa et al, 2002) and a Xror2 loss of function mutation results in defects in convergent extension movements (Schambony \& Wedlich, 2007). Although Ror proteins are highly expressed in the developing nervous system of many species, its precise role in neuronal development remains unclear (Green et al, 2008). However, Cam-1, the C. elegans homolog of Ror2 is expressed in the nervous system and vulval precursor cells and is needed for neuronal migration and axonal development as well as vulval development (Forrester et al, 1999; Green et al, 2007). The Drosophila homologs Dror and Dnrk are exclusively expressed in the nervous system, however, their precise function is still unknown (Wilson et al, 1993; Oishi et al, 1997). Dnrk has been demonstrated to genetically interact with the Dystrophin glycoprotein complex consisting of Dystroglycan and Dystrophin (Kucherenko et al, 2008; Kucherenko et al, 
2011) and plays a role in axon pathfinding and rhabdomere length in Drosophila eye development (Marrone et al, 2011).

Although Ror proteins were initially given that name because the associated ligand was unknown, it has been shown in the past years that Ror proteins interact with several Wnt ligands via their CRD (Hikasa et al, 2002; Oishi et al, 2003; Billiard et al, 2005; Mikels \& Nusse, 2006a; Green et al, 2007). Furthermore, the CRD is required for interacting with other co-receptors like Frizzled (Oishi et al, 2003). In mammals, it has been demonstrated that Ror2 acts as a receptor or co-receptor for Wnt5a and this interaction inhibits canonical signaling (Oishi et al, 2003; Mikels \& Nusse, 2006a). Similarly, Cam-1, the C. elegans Ror protein, functionally inhibits Egl-20, a C. elegans Wht protein (Kim \& Forrester, 2003; Forrester et al, 2004). In addition to suppressing canonical signal transduction, these co-receptors were shown to be able to actively promote non-canonical signaling in a Frizzled-dependent manner (Grumolato et al, 2010).

\subsubsection{Ryk / Derailed (Drl)}

Interaction between Wnt signaling and Ryk (for related to tyrosine kinase) transmembrane proteins was initially implicated to function as an axon guidance pathway (Fradkin et al, 2010). Ryks belong to the transmembrane receptor tyrosine kinase (RTK) family, however their protein kinase activity has shown to be inactive (Hovens et al, 1992; Stacker et al, 1993). Ryk family proteins have, among several other common protein motifs, an extracellular Wnt-binding Wnt-inhibitory-factor-1 (WIF) domain (Patthy, 2000) that has been shown to interact with Wnt5, but not Wnt4 or Wingless (Yoshikawa et al, 2003), (see also 1.4.4).

In Drosophila the first functional relevance of Ryk was uncovered in a screen for genes involved in neuronal pathway selection (Callahan et al, 1995). Neurons in flies homozygous mutant for the Ryk homolog derailed $(d r l)$ project along inappropriate paths (Callahan et al, 1995). Next, Derailed was demonstrated to be expressed exclusively by neurons that project in the anterior of the two commissures (anterior commissure, AC) present in each hemisegment and absence of $d r l$ leads to abnormal crossing of these neurons into the posterior commissure (PC). Conversely, ectopic expression of Drl in PC neurons forces them to cross into the AC (Bonkowsky et al, 1999). In $d r l$ mutants somatic muscles attach abnormally within the epidermis, 
indicating that similar mechanisms control muscle attachment site selection and axon pathfinding (Callahan et al, 1996).

Furthermore, the $d r l$ gene was identified in a screen for adult Drosophila learning and memory mutants; here described as linotte (lio) (Dura et al, 1993; Dura et al, 1995). lio mutants of the $d r l$ gene display structural brain defects in two adult brain centers implicated in learning and memory, the central complex and the mushroom bodies (Moreau-Fauvarque et al, 1998; Simon et al, 1998).

In vertebrates, Fz and Ryk can form a ternary complex with Wnt-1 and Wnt3a during neurite extension and axon fasciculation (Lu et al, 2004a). The C. elegans Ryk homolog Lin-18, which is required to establish polarity of the secondary vulval lineage, genetically interacts with Lin-17, the homolog of Frizzled (Sternberg \& Horvitz, 1988; Inoue et al, 2004). Furthermore, it was shown that Wnt3a-induced nuclear translocation of the Ryk intracellular domain is important for neural progenitor cell differentiation (Lyu et al, 2008). Other recent studies suggest that Ryk and Fz also function together during Xenopus convergent extension (Kim et al, 2008) as well as mammalian cortical axon repulsion ( $\mathrm{Li}$ et al, 2009). In essence, these studies revealed that Ryk not only acts as a Wnt receptor but also together with Frizzled in a variety of canonical and non-canonical pathways (Fradkin et al, 2010). The outcome of several studies characterizing different aspects of Wnt/Ryk signaling in the developing mammalian brain is that they mediate repulsive axon guidance, similarly to Drl in the embryonic Drosophila central nervous system (CNS) (Liu et al, 2005; Keeble et al, 2006; Schmitt et al, 2006).

Whereas Wnt/Ryk play a role in axon guidance during development, inappropriate activation of this pathway after injury leads to poor axon re-growth (Fradkin et al, 2010). Neither Wnts nor Ryk are expressed in the healthy adult spinal cord. However, its injury results in the induction of Wnt 1 and Wnt5a expression in the gray matter as well as increased Ryk expression in damaged axons, resulting in poor regeneration (Liu et al, 2008; Miyashita et al, 2009).

\subsubsection{Protein Tyrosine Kinase 7 (PTK7)}

The human homolog of mouse Protein tyrosine kinase 7 (PTK7) was first cloned from colon carcinoma tissue and therefore initially named Colon carcinoma kinase- 4 (CCK-4) (Mossie et al, 1995). PTK7 is a transmembrane protein with seven extracellular immunoglobulin-like domains, a single transmembrane region and a 
cytoplasmic kinase homology domain. Structurally, PTK7 therefore belongs to the receptor tyrosine kinase family; however, the molecule is catalytically inactive in all species tested so far due to a mutation in the active DFG triplet required to coordinate ATP binding during phosphorylation (chicken Kinase-like gene (KLG) (Chou \& Hayman, 1991); human PTK7 (Mossie et al, 1995); hydra Lemon (Miller \& Steele, 2000); mouse PTK7 (Jung et al, 2004)).

Loss of function of mouse PTK7 leads to defects in neural tube closure and misorientation of stereociliary bundles in the inner ear - both characteristic planar cell polarity (PCP) defects. Similarly, knockdown of PTK7 in Xenopus embryos also results in neural convergent extension and neural tube closure defects (Lu et al, 2004; Yen et al, 2009; Paudyal et al, 2010). Furthermore, PTK7 is expressed in Xenopus neural crest cells and PTK7 knockdown impairs correct neural crest cell migration (Shnitsar \& Borchers, 2008). Analysis of different transcription factor knock-out phenotypes suggested the caudal type homeobox transcription factors ( $\mathrm{Cdx}$ ) $\mathrm{Cdx} 1$ and $\mathrm{Cdx} 2$ as well as the forkhead transcription factor FoxF to be the transcriptional regulators of PTK7 during mouse development (Jakobsen et al, 2007; Savory et al, 2011).

Further characterization of PTK7 function revealed that in contrast to mouse (Yen et al, 2009), it is required for Dsh localization to the plasma membrane in Xenopus (Shnitsar \& Borchers, 2008) and that this process requires the adaptor proteins receptor of activated $C$ kinase (RACK1) and protein kinase C $\delta 1$ (PKC $\delta 1$ ) (Wehner et al, 2011). Two recent studies identified PTK7 to interact with $\beta$-catenin in Xenopus, leading to its stabilization (Puppo et al, 2011), or with Wnt proteins in both Xenopus and Drosophila, which in contrast was shown to inhibit canonical Wnt signaling (Peradziryi et al, 2011). Based on these findings, the current model for PTK7 function is that it inhibits $\beta$-catenin dependent signaling, thereby promoting PCP signaling (Fig. 10). This was further supported by an RNA interference study that identified the Drosophila PTK7 homolog off-track as a regulator of JUN $\mathrm{NH}_{2}$-terminal kinase (JNK) (Bakal et al, 2008). 


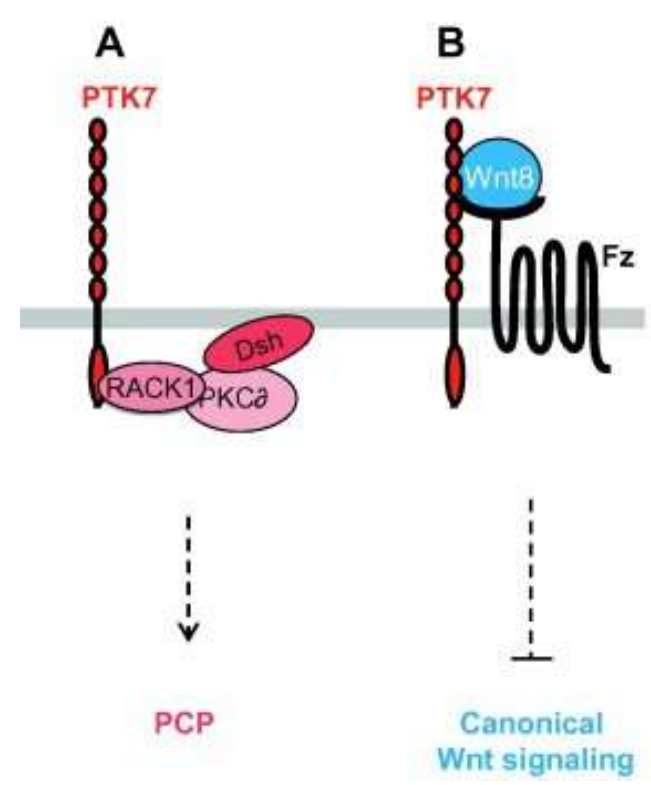

Fig. 10: Model for PTK7 function in canonical and PCP signaling.

(A) Xenopus PTK7 activates PCP signaling by recruiting Dsh to the membrane. This recruitment is mediated via interaction with RACK1 and PKC81. (B) PTK7 also functions as a Fz co-receptor in binding of canonical Wnt ligands. This interaction inhibits canonical signaling, possibly by trapping canonical Wnts. Taken from (Peradziryi et al, 2012).

\subsubsection{Off-track (Otk) - Drosophila homolog of PTK7}

PTK7 has a Drosophila homolog, the gene off-track (otk), which encodes for a glycoprotein and was first described as a neural cell adhesion molecule expressed in several areas of the developing nervous system during Drosophila embryogenesis (Pulido et al, 1992). In contrast to its homolog Ptk7 in mouse and Xenopus, mutations in this gene were not reported to display any planar cell polarity phenotypes. Instead, the $o t k^{3}$ mutant allele, removing parts of the first exon including the start codon, was reported to be homozygous lethal and to cause defects in axon pathfinding and defective targeting of photoreceptor axons to the brain (Winberg et al, 2001; Cafferty et al, 2004). Otk was demonstrated to associate with the Semaphorin 1a receptor Plexin A and therefore proposed to mediate the repulsive signaling response of Plexin A to its ligand (Winberg et al, 2001). Furthermore, it was shown that Otk is expressed in the Drosophila visual system and required for lamina-specific targeting of R1-R6 axons (Cafferty et al, 2004). Recently, it was suggested that Otk acts as a receptor for Drosophila Wnt4 in inhibiting canonical signaling (Peradziryi et al, 2011). 


\subsection{Scope of the thesis}

The importance of other Wnt transmembrane receptors apart from Frizzled has been appreciated within the past decade, but is so far not completely understood. Which pathway becomes activated is at least in part determined by the choice of Frizzled coreceptors (Angers \& Moon, 2009). Frizzled co-receptors like LRP/Arrow activate canonical signaling exclusively, while other co-receptors such as Ror and Ryk/Derailed control non-canonical signaling. PTK7/Otk also seems to belong to this group of co-receptors required for pathway selectivity (Peradziryi et al, 2012), however, the exact molecular mechanism as well as its conservation between different species remain to be clarified.

The aim of this work therefore was to investigate the role and function of Otk in Drosophila development. To this end I generated null alleles of both Drosophila Otk paralogs otk and the so far undescribed gene CG8964/otk2 and analyzed the effects of Otk loss as well as overexpression on general viability and with regard to different Wnt signaling pathways. According to the function of vertebrate PTK7, Otk/Otk2 could act as receptors of Wnt ligands and might regulate canonical or non-canoncial Wnt signaling. To investigate this possibility, the localization of Otk as well as the effect of otk/otk2 deletion was analysed with regard to different Wnt ligands as well as receptors. In this context it was intriguing to determine whether Otk might also be a target of Wnt signaling. For this purpose I analysed the expression of Otk in different wht mutant backgrounds. Furthermore, genetic interaction experiments with other Wnt receptors were performed to study if receptors might act redundantly. 


\section{MATERIALS AND METHODS}

\subsection{Materials}

\subsubsection{Chemicals and reagents}

All chemicals were of analytical grade and were purchased from Biomol (Hamburg, Germany), Bio-Rad (Munich, Germany), Biozym (Oldendorf, Germany), Difco (Detroit, USA), Fluka (Buchs, Switzerland), Gibco/BRL Life Technologies (Karlsruhe, Germany), Merck (Darmstadt, Germany), Polysciences (Eppelheim, Germany), Roth (Karlsruhe, Germany), Serva (Heidelberg, Germany), Sigma-Aldrich (Steinheim, Germany), Macherey-Nagel (Düren, Germany) if not stated otherwise. Solutions were prepared in distilled water and sterilized by autoclaving or sterile filtration.

\subsubsection{Enzymes}

Enzymes for molecular biology methods were purchased from Bioline (Luckenwalde, Germany), Fermentas (St. Leon-Rot, Germany), Genecraft (Lüdingshausen, Germany), Promega (Madison, USA), Roche (Mannheim, Germany) and used with the buffers supplies.

\subsubsection{Bacterial strains}

In this study, different Escherichia coli (E. coli) strains were used for the amplification of plasmid DNA and for the production of recombinant GST-fusion proteins. They are listed in Table 2. E. coli was grown in either lysogeny broth (LB) medium (10 g/l tryptone peptone, $5 \mathrm{~g} / \mathrm{l}$ yeast extract, $5 \mathrm{~g} / \mathrm{l} \mathrm{NaCl}$ ) or 2xYTA medium (16 g/l tryptone peptone, $15 \mathrm{~g} / \mathrm{l}$ yeast extract, $5 \mathrm{gl} \mathrm{NaCl}$ ). For production of agar plates, $2 \%$ agar-agar was added to the LB medium. It was autoclaved at $120^{\circ} \mathrm{C}$ for $30 \mathrm{~min}$ and after cooling to $60^{\circ} \mathrm{C}$, antibiotics were added $(100 \mu \mathrm{g} / \mathrm{ml}$ ampicillin, $50 \mu \mathrm{g} / \mathrm{ml}$ kanamycin).

Table 2: Bacterial strains used in this study.

\begin{tabular}{|c|c|c|}
\hline Strain & Genotype & Application \\
\hline $\mathrm{DH} 5 \alpha$ & $\begin{array}{l}\text { Ф80lacZAM15, } \Delta \text { lacZYA-argF)U169, deoR, recA1, } \\
\text { endA1, hsdR17 }\left(r_{k^{-}}, m_{k}+\right) \text {, phoA, supE44, } \lambda-\text {, thi-1, } \\
\text { gyrA96, relA1 }\end{array}$ & $\begin{array}{l}\text { Amplification of plasmid } \\
\text { DNA }\end{array}$ \\
\hline BL21 & $\mathrm{F}^{-}$, ompT, $h s d S_{B}\left(\mathrm{r}_{\mathrm{B}^{-}}, \mathrm{m}_{\mathrm{B}^{-}}-\right), d c m, g a l, \lambda(\mathrm{DE} 3)$ & $\begin{array}{l}\text { Expression of recombinant } \\
\text { proteins }\end{array}$ \\
\hline
\end{tabular}


TOP10 F'[laclq, Tn10(TetR)]mcrA $(m r r-h s d R M S-m c r B C) \quad$ Cloning of PCR fragments in Ф80lacZAM15, $\triangle$ lacX74, recA1, araD139, $\triangle$ (araleu), pENTR vector 7697 galUgalKrpsL (StrR) endA1 nupG

XL1-Blue endA1, gyrA96 $\left(n a l^{R}\right)$, thi-1, recA1, relA1, lac, glnV44, Site-directed mutagenesis $\mathrm{F}^{\prime}\left[\operatorname{Tn} 10\right.$ proAB+lacl $\left.\left.\right|^{a} \Delta(\operatorname{lacZ}) \mathrm{M} 15\right], h s d R 17\left(r_{k^{-}}, m_{k}+\right)$

\subsubsection{Oligonucleotides}

All oligonucleotides were designed using DNA-Star Lasergene V6 software (DNASTAR Inc., Madison, USA) and synthesized by Metabion (Martinsried, Germany). Common considerations for primer design were taken into account, like e.g. a base composition of 50-60\% $(\mathrm{G}+\mathrm{C})$, no primer self-complementarity and primer lengths between 17-28 bp if possible. All primers used in this study are listed in Table 3.

Table 3: Oligonucleotides used in this study.

\begin{tabular}{lll}
\hline Primer name & Sequence $\mathbf{5}^{\prime} \rightarrow \mathbf{3}^{\prime}$ & Description \\
\hline \multicolumn{2}{l}{ General sequencing primer for cloning } & \\
M13 for & GTAAACGACGGCCAG & \\
M13 rev & CAGGAAACAGCTATGAC & pENTR forward \\
ACTf & GAGCATTGCGGCTGATAAGG & pENTR reverse \\
SVr & GGCATTCCACCACTGCTCCC & pAWG and pAWM forward \\
HSPf & TATAAATAGAGGCGCTTCGT & pAWM reverse \\
EGFP-N-rev & CGGACACGCTGAACTTGTG & pTWG and pTWR forward \\
& & pAWG, pTWG and pPWG-attB \\
RFPr & GGACAGCTTCAAGTAGTCGG & reverse \\
UASPf & GGCAAGGGTCGAGTCGATAG & pPWG-attB forward
\end{tabular}

\section{Otk primer for cloning}

Otk_seq

GTGGAGGGCGCCTCAGG

Otk_seq2

CACCCTAAGCTTTGCCAGC

Otk_seq3

AGCTACGGCTGCACAATTGG

Otk_seq4

AGGCAGCGGAAGTGGAAGC

Sequencing Otk constructs in

Otk_seq5

GAGTGCATCCAGGAAGACG pENTR

otkExDomBamHI_for

CGAATGGTATCGCGGATCCGAGAAAC TTAGC

Introduction of a BamHI site in

otkExDomBamHI_rev

GCTAAGTTTCTCGGATCCGCGATACCA TTCG Otk 
otkExDomEcoRI_for $\quad \begin{aligned} & \text { GCAGAATGGCACGAATTCCTTTGCCAG } \\ & \text { C }\end{aligned}$

otkExDomEcoRI_rev

GCTGGCAAAGGAATTCGTGCCATTCT $\mathrm{GC}$
Introduction of an EcoRI site in the extracellular domain of Otk

Otk2 primer for cloning

CG8964_for_CACC

CG8964_rev+Stop

CG8964_rev-Stop
CACCATGGGGCTGAACGGAAGACG

TCACACAATATCGTCGGCCACC

CACAATATCGTCGGCCACCTGC
Cloning of Otk2 in pENTR

\section{Other cloning primers}

fz1_gate_for

fz1_gate_rev

fZ1_SEQ_for

fz1_gate_STOP_rev

fz2_gate_for

fz2_gate_rev

fZ2_SEQ_for

fz2_gate_STOP_rev

Ror_gate_for

Ror_gate_rev

Ror_SEQ_for

Ror_gate_STOP_rev
CACCATGTGGCGTCAAATCCTGTTTA

GACGTACGCCTGCGCCCGGGTCCG

CTGCATGACTGTGGAGCCCCATG

CTAGACGTACGCCTGCGCCCG

CACCATGAGACACAATCGACTGAAG

TACGTGGCTGGCCGCCGGCTGCTTGA

CCTCAACGAAGCCGTGCCGCGGACG

TCATACGTGGCTGGCCGCCGGCTGC

CACCATGAACAAATACTCGGCAT

CATTTCTGGATTACTGGCCTTAAAGT

TATCCTTGGGCATCACCATCG

TTACATTTCTGGATTACTGGCCTTA
Cloning of $\mathrm{Fz}$ in $\mathrm{pENTR}$

Cloning of Fz2 in pENTR

Cloning of Ror in pENTR

\section{Primer for characterization of transdeletion lines}

$$
\begin{aligned}
& \text { Otk_Intron1_rev1 } \\
& \text { Otk_Intron1_for1 } \\
& \text { Otk_Intron1_rev2 } \\
& \text { Otk_Intron1_for3 } \\
& \text { Otk_Intron1_rev3 } \\
& \text { Otk_Exon2_rev1 } \\
& \text { Otk_Exon2_rev2 } \\
& \text { Otk_Start_for } \\
& \text { Otk_Start_rev }
\end{aligned}
$$

GAATCGTACTGCCTGCCAAGC

GCTTGGCAGGCAGTACGATTC

GCTGTCCAAAGTTGCGTGTCC

CGACGAGGGTAAGTGGAAGAATG

CATTCTTCCACTTACCCTCGTCG

TGAGCTGGCCAAAACGGAAGC

GATTCGCACTCGAATTTCACG

CGGTTATACATATACGCAGATGACTGC

GCAGTCATCTGCGTATATGTATAACCG
Characterization of P-element lines used for the deletion of otk and afterwards for characterization of the successful otk deletion 


UpstreamEP2017_for1
UpstreamEP2017_for2
UpstreamEP2017_for3
Otk_Exon1_for1
Otk_Exon1_rev1
Otk_Exon3_rev1
Otk_Exon4_rev1
Otk_Exon5_rev1
d01360L1
e03992R3
e03992L1
e03992L2
e03992R1
e03992R2
otk_Start_for2
otk_Intron1_rev4
otk_Intron1_rev5
otk_Exon1_for2
otk_5UTR_for1
otk_Intron1_rev6
otk_Exon2_for1
otk_5UTR_for2
otk_Intron1_for2
otk_Intron1_for4
otk_Intron4_rev1
otk_Intron3_rev1
otk_Intron5_rev1
otk_Intron6_rev1
UP_inverse

CTCACGATGATCATCTTAAGTTGGTTG

C

AGCTGATTCTTGCTTTGGTCATTAAGA

CG

GACTGTCTTAGATGCACAGCAATAGG

GGATGATCTCAATATGCGGCCTGG

CCAGGCCGCATATTGAGATCATCC

CAGTTTGGCGGGCGGAGACG

GACCTGGAATGGGCGCCGAGC

CTCCCTTGTGCGAGATGGC

CTTTAAAATTCCACTTCAAACTTGC

GATTCAACTTTTTGTTGGACTCG

CACAGATAAATTGCACATCACATTACG

CACAGATAAATTGCACATCACATTACG

GTATTTATTACACTGAGCGGAAGG

TGTGTGGCCCAGAGGTGAAAGC

CATATACGCAGATGACTGCTAGG

GTTGCCTGTGTTAGTGAGCTCG

GAATCTATTAAAACAGAACG

CTGGTCATGGCTTTGATGATGG

CTAATTGAGACCAAAGGTGTCG

GTCTCACTTCAAGTGGGTATCG

CTTCCGTTTTGGCCAGCTCATCG

GTATCAGTTCGTGTGTTCTCGG

GGACACGCAACTTTGGACAGC

GCTCCTGCCCTTGCAGAGCG

GGAAGCGGTCGAAATGAAGACC

CAAGCAAAGACAGATTTGCTGTCG

CAATTGGATCTATGAAAATGC

GATTATATCAGTAGTTTGTCAGC

CATGATGAAATAACATAAGGTGGTCC

CGTC

CAATTTTACGCAGACTATCTTTCTAGG $G$

CGTACGTCACAATATGATTATCTTTCTA GG
PBac5'

PBac3'
Characterization of P-element lines used for the deletion of otk and afterwards for characterization of the successful otk deletion
Additional primers for characterization of the otk deletion 


\begin{tabular}{|c|c|c|}
\hline FRT seq1 & CGTGACTGTGCGTTAGGTCCTCC & \\
\hline XP for & AATGATTCGCAGTGGAAGGCT & \multirow{5}{*}{$\begin{array}{l}\text { Additional primers for } \\
\text { characterization of the otk } \\
\text { deletion }\end{array}$} \\
\hline FRT seq3 & CCAATTCGCCCTTGAAGATCTAGC & \\
\hline PBac_seq1 & GATATATGAAACCAATCAAAACTCG & \\
\hline PBac_seq2 & GAAACCTTTACATGAGCCTGACG & \\
\hline PBac_seq3 & CCTCGATATACAGACCGATAAAAC & \\
\hline CG8889Exon4_rev1 & CCAATATCATTGTCACCCGGCAC & \multirow{7}{*}{$\begin{array}{l}\text { Characterization of P-element } \\
\text { lines used for the deletion of } \\
\text { otk2 and otk,otk2 }\end{array}$} \\
\hline CG8889Exon3_rev1 & GTATCTGGGGATCAGCAATAAGCAGC & \\
\hline CG8889Exon5_rev1 & GTCGAGACGCGGTTATAGAGC & \\
\hline UpstreamCG8889_for1 & CATGTTTGTTTTGCTTTGAGC & \\
\hline UpstreamCG8889_for2 & GTACATGATTGCATTTCAGC & \\
\hline UpstreamCG8889_for3 & GTGCGCCCTGAAAGCGATGATGC & \\
\hline Otk5'_rev & GCAAACTCGGCTAAACAAAACG & \\
\hline CG8964_Exon2_rev1 & TGACTTGTGGAGCAGGCGTTCC & \multirow{12}{*}{$\begin{array}{l}\text { Characterization of otk } 2 \text { and } \\
\text { otk, otk } 2 \text { deletions }\end{array}$} \\
\hline CG8964_Start_for & GAAATGGGGCTGAACGGAAGACG & \\
\hline CG8964_Exon1_for & CCTGATCTTCTGCTCTTACG & \\
\hline CG8964_Exon3_for1 & GACCCATCGAGGTCGCAGAAGC & \\
\hline CG8964_Exon3_for2 & GACAAGGATCTCACCTACTTGAACG & \\
\hline CG8964_Intron1_for1 & GTGAGTTAAGTCGGCCGACTGACTCA & \\
\hline CG8964_Intron2_rev1 & GTAAGTATTAAATGTTGCATCATGC & \\
\hline CG8964_Intron2_for1 & СТTACATATAАТTСААТССТTСC & \\
\hline CG8964up5'UTR_for & CAGCGAAGTCGCTCTCATCC & \\
\hline CG8964Intron1_rev1 & GAAGAAAAGATGAGTCAGTCG & \\
\hline CG8964down3'UTR_rev & GAGCAGCGGAGGAAGTTTACG & \\
\hline PBac_seq4 & CTAGTGAAGTTCCTATTCC & \\
\hline CG7739for1 & TACACCAAGCAGAACCCACGGAG & \multirow[t]{2}{*}{ Control primer for CG7739 } \\
\hline CG7739rev2 & CCTGAGTAGTGGTACTGTAGGTG & \\
\hline
\end{tabular}

\subsubsection{Vectors and constructs}

Several constructs were generated in this study for production of fusion proteins in Escherichia coli or for transfection of cells. The basic vector backbones, to which the others go back, are listed in Table 4. The constructs that were used in this study are listed in Table 5. 
Table 4: Vectors used in this study.

\begin{tabular}{|c|c|c|}
\hline Vector name & Description & Reference \\
\hline pENTR/D-TOPO & $\begin{array}{l}\text { Entry vector for Gateway cloning, } \\
\text { Kanamycin resistance }\end{array}$ & $\begin{array}{l}\text { Invitrogen, Carlsbad, } \\
\text { Germany }\end{array}$ \\
\hline pAWG & $\begin{array}{l}\text { Expression vector, Actin } 5 \mathrm{C} \text { promoter, C- } \\
\text { terminal GFP tag, Ampicillin resistance }\end{array}$ & $\begin{array}{l}\text { Murphy lab, } \\
\text { Baltimore, USA }\end{array}$ \\
\hline pAWM & $\begin{array}{l}\text { Expression vector, Actin } 5 \mathrm{C} \text { promoter, } \mathrm{C}- \\
\text { terminal Myc tag, Ampicillin resistance }\end{array}$ & $\begin{array}{l}\text { Murphy lab, } \\
\text { Baltimore, USA }\end{array}$ \\
\hline pTWG & $\begin{array}{l}\text { Expression vector, UASt promoter, C-terminal } \\
\text { GFP tag, Ampicillin resistance }\end{array}$ & $\begin{array}{l}\text { Murphy lab, } \\
\text { Baltimore, USA }\end{array}$ \\
\hline pTWR & $\begin{array}{l}\text { Expression vector, UASt promoter, C-terminal } \\
\text { RFP tag, Ampicillin resistance }\end{array}$ & $\begin{array}{l}\text { Murphy lab, } \\
\text { Baltimore, USA }\end{array}$ \\
\hline pPWG-attB & $\begin{array}{l}\text { Expression vector, UASp promoter, C-terminal } \\
\text { GFP tag, donor attB sequence for ФC31- } \\
\text { mediated integration, Ampicillin resistance }\end{array}$ & $\begin{array}{l}\text { Murphy lab, } \\
\text { Baltimore, USA }\end{array}$ \\
\hline pGEX4T1 & $\begin{array}{l}\text { Vector for expression of GST fusion } \\
\text { proteins in E. coli, Ampicillin resistance }\end{array}$ & $\begin{array}{l}\text { Amersham Pharmacia } \\
\text { Biotech, England }\end{array}$ \\
\hline
\end{tabular}

Table 5: Constructs used in this study.

\begin{tabular}{|c|c|c|}
\hline Construct name & Description & Cloning strategy / Reference \\
\hline \multicolumn{3}{|l|}{ Otk constructs } \\
\hline Otk-pENTR & Full length Otk in pENTR & Iryna Shnitsar, unpublished \\
\hline Otk $\Delta C$-pENTR & $\begin{array}{l}\text { Otk lacking the cytoplasmic domain } \\
\text { (aa 776-1033) in pENTR }\end{array}$ & Iryna Shnitsar, unpublished \\
\hline Otk $\triangle$ Ex-pENTR & $\begin{array}{l}\text { Otk lacking the extracellular domain } \\
\text { (aa 2-474) in pENTR }\end{array}$ & Iryna Shnitsar, unpublished \\
\hline Otk-pAWG & $\begin{array}{l}\text { Full length Otk, Actin promoter, C- } \\
\text { terminal GFP-tag }\end{array}$ & $\begin{array}{l}\text { LR recombination of Otk- } \\
\text { pENTR in pAWG }\end{array}$ \\
\hline Otk-pAWM & $\begin{array}{l}\text { Full length Otk, Actin promoter, C- } \\
\text { terminal Myc-tag }\end{array}$ & $\begin{array}{l}\text { LR recombination of Otk- } \\
\text { pENTR in pAWM }\end{array}$ \\
\hline Otk $\Delta$ C-pAWM & $\begin{array}{l}\text { Otk lacking the cytoplasmic domain } \\
\text { (aa 776-1033), Actin promoter, C- } \\
\text { terminal Myc-tag }\end{array}$ & $\begin{array}{l}\text { LR recombination of Otk } \Delta C y- \\
\text { pENTR in PAWM }\end{array}$ \\
\hline Otk $\Delta$ Ex-pAWM & $\begin{array}{l}\text { Otk lacking the extracellular domain } \\
\text { (aa 2-474), Actin promoter, C-terminal } \\
\text { Myc-tag }\end{array}$ & $\begin{array}{l}\text { LR recombination of Otk } \Delta \text { Ex- } \\
\text { pENTR in PAWM }\end{array}$ \\
\hline Otk-pPWG-attB & $\begin{array}{l}\text { Full length Otk, UASp promoter, C- } \\
\text { terminal GFP tag, attB donor site }\end{array}$ & $\begin{array}{l}\text { LR recombination of Otk- } \\
\text { pENTR in pPWG-attB }\end{array}$ \\
\hline Otk $\Delta$ C-pPWG-attB & $\begin{array}{l}\text { Otk lacking the cytoplasmic domain } \\
\text { (aa 776-1033), UASp promoter, C- } \\
\text { terminal GFP tag, attB donor site }\end{array}$ & $\begin{array}{l}\text { LR recombination of Otk } \Delta C y- \\
\text { pENTR in pPWG-attB }\end{array}$ \\
\hline
\end{tabular}




\begin{tabular}{|c|c|c|}
\hline Otk $\Delta$ Ex-pPWG-attB & $\begin{array}{l}\text { Otk lacking the extracellular domain } \\
\text { (aa 2-474), UASp promoter, C- } \\
\text { terminal GFP tag, attB donor site }\end{array}$ & $\begin{array}{l}\text { LR recombination of Otk } \Delta E x- \\
\text { pENTR in pPWG-attB }\end{array}$ \\
\hline Otk-pTWG & $\begin{array}{l}\text { Full length Otk, UASt promoter, C- } \\
\text { terminal GFP tag }\end{array}$ & $\begin{array}{l}\text { LR recombination of Otk- } \\
\text { pENTR in } p T W G\end{array}$ \\
\hline Otk-pENTR+BamHI & $\begin{array}{l}\text { Otk-pENTR with BamHI site } \\
\text { introduced into extracellular domain }\end{array}$ & $\begin{array}{l}\text { Mutagenesis of Otk-pENTR } \\
\text { with otkExDomBamHI_for/rev }\end{array}$ \\
\hline $\begin{array}{l}\text { Otk- } \\
\text { pENTR+BamHI+EcoRI }\end{array}$ & $\begin{array}{l}\text { Otk-pENTR with BamHI and EcoRI } \\
\text { sites introduced into extracellular } \\
\text { domain }\end{array}$ & $\begin{array}{l}\text { Mutagenesis of Otk-pENTR } \\
\text { +BamHI with } \\
\text { otkExDomEcoRI_for/rev }\end{array}$ \\
\hline OtkEx-pGEX4T1 & $\begin{array}{l}\text { Extracellular domain of Otk (aa 159- } \\
338 \text { ) in pGEX4T1 }\end{array}$ & $\begin{array}{l}\text { Otk-pENTR+BamHI+EcoRI cut } \\
\text { with EcoRI and BamHI, } \\
\text { fragment ligated in pGEX4T1 }\end{array}$ \\
\hline Otk-pOT2 & $\begin{array}{l}\text { CDNA clone containing full length Otk, } \\
\text { binding sites for SP6 and T7 }\end{array}$ & LP17455 (DGRC) \\
\hline \multicolumn{3}{|l|}{ Otk2 constructs } \\
\hline Otk2-pENTR & Full length Otk2 in pENTR & $\begin{array}{l}\text { Amplification of Otk2 coding } \\
\text { sequence with CG8964_for_- } \\
\text { CACC / CG8964_rev-Stop and } \\
\text { introduction of PCR product in } \\
\text { pENTR }\end{array}$ \\
\hline Otk2-pAWG & $\begin{array}{l}\text { Full length Otk2, Actin promoter, C- } \\
\text { terminal GFP-tag }\end{array}$ & $\begin{array}{l}\text { LR recombination of Otk2- } \\
\text { pENTR in pAWG }\end{array}$ \\
\hline Otk2-pAWM & $\begin{array}{l}\text { Full length Otk2, Actin promoter, C- } \\
\text { terminal Myc-tag }\end{array}$ & $\begin{array}{l}\text { LR recombination of Otk2- } \\
\text { pENTR in pAWM }\end{array}$ \\
\hline Otk2-pPWG-attB & $\begin{array}{l}\text { Full length Otk2, UASp promoter, C- } \\
\text { terminal GFP tag, attB donor site }\end{array}$ & $\begin{array}{l}\text { LR recombination of Otk2- } \\
\text { pENTR in pPWG-attB }\end{array}$ \\
\hline Otk2-pTWR & $\begin{array}{l}\text { Full length Otk2, UASt promoter, C- } \\
\text { terminal RFP tag }\end{array}$ & $\begin{array}{l}\text { LR recombination of Otk2- } \\
\text { pENTR in pTWR }\end{array}$ \\
\hline Otk2-pFLCl & $\begin{array}{l}\text { cDNA clone containing full length Otk, } \\
\text { binding sites for T3 and T7 }\end{array}$ & DGRC (RE41180) \\
\hline
\end{tabular}

\section{Other constructs}

Fz1-pENTR Full length Fz1 in pENTR

Fz1-pAWM

Fz2-pENTR

Fz2-pAWM
Full length Fz1, Actin promoter, Cterminal Myc-tag

Full length Fz2 in pENTR

Full length Fz2, Actin promoter, Cterminal Myc-tag
Amplification of Fz1 coding sequence with fz1_gate_for/ rev and introduction of PCR product in pENTR

$L R$ recombination of Fz1pENTR in pAWM

Amplification of $\mathrm{Fz} 2$ coding sequence with $f z 2$ gate_for/ rev and introduction of PCR product in pENTR

$L R$ recombination of Fz2pENTR in pAWM 


\begin{tabular}{lll} 
Ror-pENTR & Full length Ror in pENTR & $\begin{array}{l}\text { Amplification of Ror coding } \\
\text { sequence with Ror_gate_for/ } \\
\text { rev and introduction of PCR } \\
\text { product in pENTR }\end{array}$ \\
Ror-pAWM & Full length Fz1, Actin promoter, C- & $\begin{array}{l}\text { LR recombination of Ror- } \\
\text { pENTR in pAWM }\end{array}$ \\
\hline
\end{tabular}

\subsubsection{Antibodies}

Antibodies used in this study are listed in Table 6 and Table 7 and were used at the indicated dilutions.

To raise polyclonal antibodies against Off-track (Otk), a GST fusion protein corresponding to aa 159-338 of the extracellular domain was purified and injected into guinea pigs (Eurogentec, Seraing, Belgium). The final bleed of guinea pig 336 was used for all experiments described in this study. To generate peptide antibodies against Off-track2 (Otk2, CG8964), the peptides VELGRMDSTTSEPQLE (aa 93-98, internal fragment, EP104043) and ESTILEQESQVADDIV (aa 418-433, at C-terminus, EP104042) were used to inject into rabbits (Eurogentec, Seraing, Belgium). The serum affinity purified against the C-terminal peptide EP104042 was used for all experiments described in this study.

Table 6: Primary antibodies used in this study.

IF - Immunofluorescence, IP - Immunoprecipitation WB - Western Blot, DSHB - Developmental Studies Hybridoma Bank, University of Iowa, USA.

\begin{tabular}{llclll}
\hline Epitope & Animal & Application & Dilution & Designation & Source \\
\hline Actin & Rabbit & WB & $1: 2000$ & A2066 & Sigma \\
Bazooka & Rabbit & IF & $1: 1000$ & DE99646-2 & Wodarz et al, 2000 \\
BP 102 & Mouse & IF & $1: 50$ & BP 102 & DSHB \\
B-Galactosidase & Mouse & WB,IP & $1: 200$ & JIE7 & DSHB \\
C-Myc & Mouse & WB,IP & $1: 200$ & 9E10 & DSHB \\
DE-Cadherin & Rat & IF & $1: 5$ & DCAD 2 & DSHB \\
Elav & Mouse & IF & $1: 50$ & 9F8A9 & DSHB \\
Fasciclin III & Mouse & IF & $1: 20$ & 7 G10 & DSHB \\
GFP & Mouse & IF,WB & $1: 1000$ & A11120 & Molecular Probes \\
GFP & Rabbit & IF,WB & $1: 1000$ & A11121 & Molecular Probes \\
GST & Rabbit & WB & $1: 20000$ & G7781 & Sigma \\
Miranda & Guinea Pig & IF & $1: 1000$ & DE02120 & Wodarz, \\
& & & & & unpublished \\
Otk & Guinea Pig & WB,IF & $1: 1000$ & DE11243 & This work \\
& & & & SAC336 & \\
Otk2 & Rabbit & WB,IF & $1: 100$ & EP104042 & This work
\end{tabular}




\begin{tabular}{llclll}
\hline Tubulin $\alpha$ & Mouse & WB & $1: 1000$ & $12 \mathrm{G} 10$ & DSHB \\
Vasa & Rabbit & IF & $1: 2000$ & & Ruth Lehmann \\
Wingless & Mouse & IF & $1: 20$ & 4D4 & DSHB \\
Wnt5 & Rabbit & IF & $1: 150$ & & Fradkin et al, 2004 \\
\hline
\end{tabular}

Table 7: Secondary antibodies used in this study.

IF - Immunofluorescence, WB - Western Blot.

\begin{tabular}{llclll}
\hline Epitope & Conjugate & Application & Dilution & Origin & Source \\
\hline Guinea Pig IgG & HRP & WB & $1: 10000$ & & Dianova \\
Mouse IgG & HRP & WB & $1: 10000$ & Goat & Dianova \\
Rabbit IgG & HRP & WB & $1: 10000$ & Goat & Dianova \\
Guinea Pig IgG & Cy2, Cy3 & IF & $1: 200$ & Donkey & Dianova \\
Guinea Pig IgG & Cy5 & IF & $1: 200$ & Goat & Dianova \\
Mouse IgG & Cy2, Cy3 & IF & $1: 200$ & Donkey & Dianova \\
Mouse & Cy5 & IF & $1: 200$ & Goat & Dianova \\
Rabbit IgG & Cy2, Cy5 & IF & $1: 200$ & Goat & Dianova \\
Rabbit IgG & Cy3 & IF & $1: 200$ & Donkey & Dianova \\
\hline
\end{tabular}

\subsubsection{Fly stocks}

Table 8: Fly stocks used in this study.

BL - Bloomington Stock Center.

\begin{tabular}{|c|c|c|c|}
\hline Stock & Genotype & Description & Reference \\
\hline wild type oregonR & wild type & Red eyes & $\begin{array}{l}\text { Stock collection } \\
\text { Wodarz }\end{array}$ \\
\hline white- & $w^{1118}$ & White eyes & BL 5905 \\
\hline Gla/CyO(ftz::lacZ) & Gla/CyO, P(ftz::lacZ) & $\begin{array}{l}\text { Balancer } 2^{\text {nd }} \\
\text { chromosome }\end{array}$ & $\begin{array}{l}\text { Stock collection } \\
\text { Wodarz }\end{array}$ \\
\hline $\begin{array}{l}\text { TM3(ftz::lacZ)/ } \\
\text { TM6B }\end{array}$ & $\begin{array}{l}\text { w;;TM3(ftz::lacZ)ce, } \\
\text { Ser/TM6B, e, Tu, Ser }\end{array}$ & $\begin{array}{l}\text { Balancer } 3^{\text {rd }} \\
\text { chromosome }\end{array}$ & $\begin{array}{l}\text { Stock collection } \\
\text { Wodarz }\end{array}$ \\
\hline $\begin{array}{l}\text { If/CyO; } \\
\text { MKRS/TM6B }\end{array}$ & $w ;$ If/CyO; MKRS/TMGB & $\begin{array}{l}\text { Balancer } 2^{\text {nd }} \text { and } 3^{\text {rd }} \\
\text { chromosome }\end{array}$ & $\begin{array}{l}\text { Stock collection } \\
\text { Wodarz }\end{array}$ \\
\hline da-Gal4 & $\begin{array}{l}w\left[^{*}\right] ; P\{w[+m W . h s] \\
=G A L 4-d a . G 32\} U H 1\end{array}$ & $\begin{array}{l}\text { Gal4 driver line, } \\
\text { ubiquitous expression in } \\
\text { daughterless pattern, } \\
3^{\text {rd }} \text { chromosome }\end{array}$ & BL 5460 \\
\hline PhiC31 86FB & $\begin{array}{l}\mathrm{P}\{\mathrm{ry}[+\mathrm{t} 7.2]=\text { hsp70-flp\}1, } \\
\mathrm{y}[1] \mathrm{w}[*] ; \mathrm{M}\{3 \times \mathrm{xP} 3- \\
\text { RFP.attP\}ZH-86Fb; M\{vas- } \\
\text { int.B\}ZH-102D }\end{array}$ & $\begin{array}{l}\text { phic31 integrase } \\
\text { expressed from the vasa } \\
\text { promoter; 3rd } \\
\text { chromosome attP site }\end{array}$ & BL23648 \\
\hline ywhsFlp;Sco/CyO & $\begin{array}{l}\mathrm{P}\{\mathrm{ry}[+\mathrm{t} 7.2]=\mathrm{hsFLP}\} 12, \mathrm{y}[1] \\
\mathrm{w}\left[^{*}\right] ; \text { sna[Sco]/CyO }\end{array}$ & $\begin{array}{l}\text { Heat shock FLP stock } \\
\text { used for transdeletion }\end{array}$ & BL1929 \\
\hline
\end{tabular}




\begin{tabular}{|c|c|c|c|}
\hline otk $k^{\mathrm{CPTI} 1000252}$ & $\begin{array}{l}\text { PBac\{602.P.SVS- } \\
1\} o t k^{\text {CPTI000252 }}\end{array}$ & GFP trap otk & Kyoto stock center \\
\hline CG8964 $4^{\text {SH1639 }}$ & $P\{\mid a c W\} I(2) S H 1639^{S H 1639}$ & $\begin{array}{l}\text { LacZ enhancer trap } \\
\text { CG8964 }\end{array}$ & Kyoto stock center \\
\hline$P(X P) d 01360$ & $P\{X P\} d 01360$ & $\begin{array}{l}\text { P-element insertion } \\
\text { upstream of otk }\end{array}$ & $\begin{array}{l}\text { Exelixis collection, } \\
\text { Harvard }\end{array}$ \\
\hline PBac(PB)c01790 & $P B a c\{P B\} M p p e^{c 01790}$ & $\begin{array}{l}\text { P-element insertion } \\
\text { upstream of otk } 2\end{array}$ & $\begin{array}{l}\text { Exelixis collection, } \\
\text { Harvard }\end{array}$ \\
\hline PBac(RB)e03992 & PBac $\{R B\} e 03992$ & $\begin{array}{l}\text { P-element insertion } \\
\text { downstream of otk }\end{array}$ & $\begin{array}{l}\text { Exelixis collection, } \\
\text { Harvard }\end{array}$ \\
\hline Df(3R)BSC39 & $\begin{array}{l}\mathrm{Df}(2 \mathrm{R}) \mathrm{BSC} 39, \mathrm{cn}[1] \\
\mathrm{bw}[1] / \mathrm{SM} 6 \mathrm{a}, \mathrm{bw}[\mathrm{k} 1]\end{array}$ & $\begin{array}{l}\text { chromosomal deletion } \\
\text { removing otk and otk } 2\end{array}$ & BL 7145 \\
\hline Df(3R)BSC199 & $\begin{array}{l}\text { w[1118]; } \\
\text { Df(2R)BSC199/CyO }\end{array}$ & $\begin{array}{l}\text { chromosomal deletion } \\
\text { removing otk and otk2 }\end{array}$ & BL 9626 \\
\hline$o t k^{A 1}$ & $w ;$ otk $^{A 1}$ & Null alele otk & This work \\
\hline$o t k^{C 26}$ & $w ;$ otk $^{\mathrm{C26}}$ & Null allele otk2 & This work \\
\hline otk,otk $2^{D 72}$ & $w ; o t k, o t k 2^{D 72} / C y O$ & Null allele otk and otk2 & This work \\
\hline UASp::Otk-GFP29 & $w ; ;$ UASp::Otk-GFP29 & $\begin{array}{l}\text { Transgenic line after } \\
\text { injection of Otk-pPWG- } \\
\text { attB in BL } 23648\end{array}$ & This work \\
\hline $\begin{array}{l}\text { UASp::Otk } \Delta \mathrm{C}- \\
\text { GFP14 }\end{array}$ & $w ; ;$ UASp::Otk $\Delta$ C-GFP14 & $\begin{array}{l}\text { Transgenic line after } \\
\text { injection of Otk } \Delta C \text { - } \\
\text { pPWG-attB in BL } 23648\end{array}$ & This work \\
\hline $\begin{array}{l}\text { UASp::Otk } \Delta \mathrm{Ex}- \\
\text { GFP20 }\end{array}$ & $w ; ;$ UASp::Otk $\Delta \mathrm{Ex}-\mathrm{GFP} 20$ & $\begin{array}{l}\text { Transgenic line after } \\
\text { injection of Otk } \Delta \text { Ex- } \\
\text { pPWG-attB in BL } 23648\end{array}$ & This work \\
\hline UASt::OtkGFP & $w ; ;$ UASt::OtkGFP & $\begin{array}{l}\text { Transgenic line after } \\
\text { injection of Otk-pTWG } \\
\text { in white- }\end{array}$ & This work \\
\hline $\begin{array}{l}\text { UASp::Otk2- } \\
\text { GFP37 }\end{array}$ & w;; UASp::Otk2-GFP37 & $\begin{array}{l}\text { Transgenic line after } \\
\text { injection of Otk2-pPWG- } \\
\text { attB in BL } 23648\end{array}$ & This work \\
\hline UASt::Otk2-RFP & w;; UASt::Otk2-RFP & $\begin{array}{l}\text { Transgenic line after } \\
\text { injection of Otk2-pTWR } \\
\text { in white- }\end{array}$ & This work \\
\hline$w g^{C X 4}$ & $w g[l-17] b[1] \operatorname{pr}[1] / C y O$ & wg null allele & BL 2980 \\
\hline$w n t 4^{E M S S 23}$ & $\begin{array}{c}w n t 4[E M S 23] b w[1] / C y O, \\
P\{r y[+t 7.2]=H B-l a c Z\} G S 1\end{array}$ & wnt4 null allele & BL 6650 \\
\hline$w n t 4^{C 1}$ & $w[1118] ;$ Wnt4[C1]/CyO & wnt4 null allele & BL 6651 \\
\hline$w n t 2^{L}$ & $\begin{array}{l}\text { w[1118]; Wnt2[L]/ } \\
\text { CyO, amos[Roi-1] }\end{array}$ & wnt2 null allele & BL 6909 \\
\hline
\end{tabular}




\begin{tabular}{|c|c|c|c|}
\hline$w n t 2^{\circ}$ & $\begin{array}{l}\text { Wnt2[O]/CyO, amos } \\
\text { [Roi-1] }\end{array}$ & wnt2 null allele & BL 6958 \\
\hline$w n t 2^{\prime}$ & $\begin{array}{l}\text { Wnt2[I]/CyO, amos } \\
\text { [Roi-1] }\end{array}$ & wnt2 null allele & BL 6960 \\
\hline$w n t 2^{R J}$ & $\begin{array}{l}\text { Wnt2[RJ]/CyO, amos[Roi- } \\
\text { 1] }\end{array}$ & wnt2 null allele & BL 6959 \\
\hline$D f(2 L) D E$ & $\begin{array}{l}w[1118] ; D f(2 L) D E / C y O, \\
P\{r y[+t 7.2]=f t z / l a c B\} E 3\end{array}$ & $\begin{array}{l}\text { chromosomal deletion } \\
\text { removing } w g \text { and wnt } 4\end{array}$ & BL 6653 \\
\hline$w n t 4^{C 1}, w n t 2^{L}$ & $\begin{array}{l}y[1] w[1118] ; \text { Wnt4[C1] } \\
\text { Wnt2[L]/CyO-Df(2R) } \\
B 80, y[+]\end{array}$ & $\begin{array}{l}\text { deletion of wnt4 and } \\
\text { wnt2 }\end{array}$ & BL 6907 \\
\hline$w g^{c \times 4}, w n t 2^{L}$ & $w g[l-17]$ Wnt2[L]/CyO & deletion of $w g$ and $w n t 2$ & BL 6908 \\
\hline$w n t 5^{400}$ & & wnt5 null allele & Fradkin et al, 2004 \\
\hline$d s h^{1}$ & $y w d s h^{1} / Y / C(1)$ & $\begin{array}{l}d s h^{1} \text { allele, disrupts } \\
\text { tissue polarity but not } \\
\text { Wg signaling }\end{array}$ & $\begin{array}{l}\text { Stock collection } \\
\text { Wodarz }\end{array}$ \\
\hline$f z^{J 22}$ & $\mathrm{fz}^{\mathrm{J22}} / \mathrm{TM} 6 \mathrm{C}$ & $f z$ allele, autonomous & Paul Adler \\
\hline$f z^{H 51}$ & $\mathrm{fz}^{\mathrm{H} 51}$ th st/TM6C & $\begin{array}{l}f z \text { allele, non- } \\
\text { autonomous }\end{array}$ & Paul Adler \\
\hline$f z^{R 52}$ & $\mathrm{fz}^{R 52}$ th st/TM6C & $\begin{array}{l}f z \text { allele, non- } \\
\text { autonomous }\end{array}$ & Ken Cadigan \\
\hline$f z^{P 21}$ & $\mathrm{fz}^{P 21}$ th st/TM6C & $\begin{array}{l}f z \text { allele, non- } \\
\text { autonomous }\end{array}$ & Paul Adler \\
\hline$f z^{R 52} D f(3 L) D f z 2$ & $\begin{array}{l}w ; ; f z^{R 52} D f(3 L) D f z 2 \\
P[w+F R T 2 A] / T M 6\end{array}$ & $f z, D f z 2$ double mutant & Ken Cadigan \\
\hline ProtB-EGFP & w;;protamineB-eGFP & & $\begin{array}{l}\text { Raja \& Renkawitz- } \\
\text { Pohl, } 2005\end{array}$ \\
\hline
\end{tabular}

\subsection{Molecular biology methods}

If not indicated otherwise, standard procedures were carried out as described in the manufacturer's protocol or in "Molecular Cloning: A Laboratory Manual" (Sambrook \& Russell, 2000).

\subsubsection{Polymerase chain reaction (PCR)}

Polymerase chain reaction allows the specific amplification of DNA fragments in vitro. In general, PCR reactions were done in $50 \mu$ l total reaction volume. $1 \mu$ l plasmid DNA (corresponding to 20 - $50 \mathrm{ng}$ ) or 5-10 $\mu$ l fly genomic DNA (corresponding to $200 \mathrm{ng}$ ) were mixed with $400 \mathrm{nM}$ of forward and reverse primer (Table 3), $250 \mu \mathrm{M}$ of each dNTP (Bioline) and $1 \mathrm{U}$ polymerase in the corresponding buffer (see Table 9 for a standard pipetting scheme). The primers used in this study are listed in Table 3. PCR 
reactions were performed with Taq polymerase (Genecraft) or Pfu polymerase (Bioline). For PCR reactions in a smaller final volume the reaction volumes were adjusted accordingly (Table 9). A standard PCR program is depicted in Table 10.

Table 9: Standard PCR.

\begin{tabular}{lll}
\hline Template DNA & $1 \mu \mathrm{l}(20-50$ ng plasmid DNA $)$ & $2 \mu \mathrm{l}(200$ ng genomic DNA) \\
Forward Primer $(10 \mu \mathrm{M})$ & $2 \mu \mathrm{l}(200$ ng genomic DNA $)$ & \\
Reverse Primer $(10 \mu \mathrm{M})$ & $2 \mu \mathrm{l}$ & $0,5 \mu \mathrm{l}$ \\
dNTPs (25 mM each) & $0,5 \mu \mathrm{l}, 5 \mu \mathrm{l}$ \\
10X polymerase buffer & $5 \mu \mathrm{l}$ & $0,2 \mu \mathrm{l}$ \\
Polymerase & $0,5 \mu \mathrm{l}$ & $2 \mu \mathrm{l}$ \\
ddH2O & ad $\mathbf{5 0} \mu \mathrm{l}$ & $0,2 \mu \mathrm{l}$ \\
\hline
\end{tabular}

Table 10: Standard PCR program.

\begin{tabular}{|c|c|c|c|}
\hline Step & Temperature & Duration & \\
\hline 1) Initial denaturation & $95^{\circ} \mathrm{C}$ & $5 \mathrm{~min}$ & \\
\hline 2) Denaturation & $95^{\circ} \mathrm{C}$ & $30 \mathrm{sec}$ & \\
\hline 3) Annealing & $52-65^{\circ} \mathrm{C}$ & $30 \mathrm{sec}$ & $5-10^{\circ} \mathrm{C}$ below primer $\mathrm{Tm}$ \\
\hline 4) Elongation & $72^{\circ} \mathrm{C}$ & $\begin{array}{l}1,5 \mathrm{~min} / \mathrm{kb}(\mathrm{Pfu}) \\
0,5-1 \mathrm{~min} / \mathrm{kb} \text { (Taq) }\end{array}$ & \\
\hline \multicolumn{4}{|c|}{ Repeat steps 2-4 34 times } \\
\hline \multirow[t]{2}{*}{ 5) Final elongation } & $72^{\circ} \mathrm{C}$ & $5 \mathrm{~min}$ & \\
\hline & $4^{\circ} \mathrm{C}$ & Hold & \\
\hline
\end{tabular}

The annealing temperature was chosen based on the melting temperature as indicated by the supplier.

\subsubsection{Restriction digestion of DNA}

Plasmid DNA or PCR products were subjected to site-specific restriction digestion in order to obtain DNA fragments containing the desired sequence. Restriction digestion was carried out according to manufacturer's instructions.

\subsubsection{Agarose gel electrophoresis}

DNA fragments obtained by PCR or restriction digestion products were separated electrophoretically on ethidium bromide-containing agarose gels $(1 \% \mathrm{w} / \mathrm{v}$ agarose, $40 \mathrm{mM}$ Tris, $10 \mathrm{mM}$ EDTA, 0,5 $\mathrm{gg} / \mathrm{ml}$ ethidium bromide). DNA samples were mixed with $1 / 6$ volume of DNA loading buffer (Fermentas), loaded and separated at $100 \mathrm{~V}$ for 20 - 30 min. To determine the size of separated DNA fragments, GeneRuler $1 \mathrm{~kb}$ 
DNA Ladder (Fermentas) was loaded in a separate pocket. DNA bands were visualized with UV light.

\subsubsection{DNA extraction from agarose gels}

DNA was extracted from agarose gels using the NucleoSpin Extract II Kit (MacheryNagel) according to manufacturer's instructions.

\subsubsection{Ligation of DNA fragments}

DNA inserts and vector backbones that have been digested with suitable restriction enzymes were ligated using T4 DNA ligase (Fermentas). If only one enzyme was used for cloning, vector backbones were incubated with calf intestine phosphatase (CIAP) (Fermentas) for $30 \mathrm{~min}$ at $37^{\circ} \mathrm{C}$, followed by a purification step. Calf intestine phosphatase removes $5^{\prime}$ phosphate groups from DNA and thus prevents vector selfligation.

\subsubsection{Generation of expression constructs by Gateway cloning technology}

To express full length and partially deleted versions of genes of interest in S2r+ cells and transgenic flies, the corresponding coding regions were amplified with primers indicated in (Table 3). The PCR products were cloned into pENTR/D-TOPO vector (Invitrogen) according to manufacturer's instructions. The inserts of the corresponding pENTR constructs were recombined into destination vectors of the Drosophila Gateway Vector Collection (Carnegie Institution of Washington, Baltimore, MD) by $\lambda$ phage mediated LR recombination. The LR recombination was performed with LR Clonase Enzyme Mix (Invitrogen) according to the manufacturer's protocol, using $100 \mathrm{ng}$ of pENTR, $150 \mathrm{ng}$ of destination vector and 0,5 $\mu \mathrm{l}$ LR Clonase mix.

\subsubsection{Transformation of bacteria}

For plasmid DNA amplification, the plasmid or the ligation/pENTR/Clonase reaction was used to transform chemically competent bacteria. E. coli strains used in this work and their applications are listed in Table 2. Briefly, competent cells were thawed on ice, approximately 100 ng of plasmid DNA was added, gently mixed and incubated on ice for $30 \mathrm{~min}$. Then the cells were heat-shocked at $42{ }^{\circ} \mathrm{C}$ for $45 \mathrm{sec}$, followed by incubation on ice for $2 \mathrm{~min}$ and recovery with warm SOC medium (2\% tryptone, $0,5 \%$ yeast extract, $10 \mathrm{mM} \mathrm{NaCl}, 2,5 \mathrm{mM} \mathrm{KCl}, 10 \mathrm{mM} \mathrm{MgCl} 2,10 \mathrm{mM} \mathrm{MgSO}$, 20mM glucose) at 
$37{ }^{\circ} \mathrm{C}$ for $30-60 \mathrm{~min}$. The cells were streaked on LB agar plates (1\% tryptone, $0,5 \%$ yeast extract, $1 \% \mathrm{NaCl}, 1 \%$ agar) containing antibiotic for selection (see 2.1.3) of transformed cells and cultured overnight at $37^{\circ} \mathrm{C}$.

\subsubsection{Plasmid purification from bacteria}

To purify plasmid DNA from E. coli cells in smaller amounts, plasmid DNA was purified according to a modified plasmid purification method from Qiagen, Germany. Briefly, a single colony of transformed E. coli cells was inoculated in $2 \mathrm{ml}$ LB medium containing the appropriate antibiotic for selection $(100 \mu \mathrm{g} / \mathrm{ml}$ ampicillin, $50 \mu \mathrm{g} / \mathrm{ml}$ kanamycin). After incubation overnight at $37^{\circ} \mathrm{C}$ with constant agitation, the cells were pelleted by centrifugation for $1 \mathrm{~min}$ at $13000 \mathrm{rpm}$. The pellet was resuspended in $200 \mu \mathrm{l}$ of buffer P1 (50mM Tris-HCl, pH 8,0. 10mM EDTA, $100 \mu \mathrm{g} / \mathrm{ml}$ RNase A). Afterwards $200 \mu \mathrm{l}$ of buffer P2 (200mM NaOH, 1\% SDS) was added, the mixture was mixed by inverting 5-6 times and incubated for $5 \mathrm{~min}$ at room temperature. After addition of $200 \mu \mathrm{l}$ of buffer P3 (3M K acetate, $\mathrm{pH}$ 5,5) the mixture was inverted 5-6 times and centrifuged for $20 \mathrm{~min}$ at $13000 \mathrm{rpm}, 4{ }^{\circ} \mathrm{C}$. The supernatant was transferred to a fresh centrifuge tube and DNA was precipitated with $400 \mu \mathrm{l}$ of isopropanol for $30 \mathrm{~min}$ at $13000 \mathrm{rpm}, 4^{\circ} \mathrm{C}$. The resulting DNA pellet was washed with $200 \mu \mathrm{l}$ ice-cold 70\% ethanol and subsequently air-dried at room temperature. Purified plasmid DNA was dissolved in $20 \mu \mathrm{lddH_{2 }}$ O.

To obtain large amounts of pure DNA for transfection and injection of flies, plasmid DNA was purified with Nucleobond X100 kit (Macherey-Nagel) according to the manufacturer's procedure.

\subsubsection{Long-term storage of bacteria}

E. coli can be stored in glycerol stocks at $-80^{\circ} \mathrm{C}$ for several years. Glycerol stocks were prepared by adding $200 \mu \mathrm{l} 99 \%$ glycerol to $800 \mu \mathrm{l}$ of a logarithmic-phase E. coli culture in a cyrotube vial. They were mixed by inverting them, frozen in liquid nitrogen and stored at $-80^{\circ} \mathrm{C}$. For recovery, parts of the stocks were scratched with a fresh pipette tip and cultured in LB medium containing the appropriate antibiotic.

\subsubsection{Site-directed mutagenesis}

Mutageneses were performed to create point mutation in a sequence of interest. In brief, mutagenesis was done by allowing Pfu polymerase to synthesize the complete 
vector based on two mutagenesis primers containing the desired base pair exchanges. Pfu polymerase exhibits $3^{\prime} \rightarrow 5^{\prime}$ proofreading activity. Therefore whole vectors can be synthesized with high fidelity. The primers are complementary to opposite strands of the vector and the newly synthesized vector will contain the mutation. The PCR program used is shown in Table 11. Methylated parental DNA was digested with DpnI endonuclease. The remaining mutation-containing DNA was transformed into chemically competent bacteria as described in 2.2.7.

Table 11: PCR program for site-directed mutagenesis.

\begin{tabular}{lll}
\hline Step & Temperature & Duration \\
\hline 1) Initial denaturation & $95^{\circ} \mathrm{C}$ & $30 \mathrm{sec}$ \\
2) Denaturation & $95^{\circ} \mathrm{C}$ & $30 \mathrm{sec}$ \\
3) Annealing & $55^{\circ} \mathrm{C}$ & $1 \mathrm{~min}$ \\
4) Elongation & $68^{\circ} \mathrm{C}$ & $1 \mathrm{~min} / \mathrm{kb}$ \\
\multicolumn{2}{c}{ Repeat steps $2-412-18$ times } \\
5) Final elongation & $37^{\circ} \mathrm{C}$ & Hold
\end{tabular}

Point mutation: 12 cycles; single amino acid change: 16 cycles; deletion or insertion of multiple amino acids: 18 cycles.

\subsubsection{Sequencing of DNA}

Sequencing reations were set up as indicated in Table 12. The PCR program for the sequencing reaction is shown in Table 13. After completion, DNA was precipitated. Briefly, $1 \mu \mathrm{l}$ of $125 \mathrm{mM}$ EDTA, $1 \mu \mathrm{l}$ of $3 \mathrm{M} \mathrm{NaAc}$ and $50 \mu \mathrm{l}$ of $100 \%$ ethanol was added to the sample and incubated for $5 \mathrm{~min}$ at room temperature. Then the sample was centrifuged for $15 \mathrm{~min}$ at $13000 \mathrm{rpm}$, the supernatant was removed and the pellet washed with $70 \%$ ethanol and subsequently air-dried. Afterwards the pellet was dissolved in $15 \mu$ l of HiDi (Applied Biosystems). Analysis of sequencing reactions was done by an in-house sequencing service in the Department of Developmental Biochemistry, Ernst-Caspari-Haus, GZMB, Göttingen.

Table 12: Sequencing PCR.

\begin{tabular}{lll}
\hline Template DNA & $300 \mathrm{ng}$ plasmid DNA & $20-30 \mathrm{ng}$ PCR \\
Forward Primer $(10 \mu \mathrm{M})$ & $0,8 \mu \mathrm{l}$ & $0,8 \mu \mathrm{l}$ \\
SeqMix & $1,5 \mu \mathrm{l}$ & $1 \mu \mathrm{l}$ \\
SeqBuffer & $1,5 \mu \mathrm{l}$ & $1 \mu \mathrm{l}$ \\
ddH2O & ad $10 \mu \mathrm{l}$ & ad $10 \mu \mathrm{l}$ \\
\hline
\end{tabular}


Table 13: Sequencing PCR program.

\begin{tabular}{lll}
\hline Step & Temperature & Duration \\
\hline 1) Initial denaturation & $96^{\circ} \mathrm{C}$ & $2 \mathrm{~min}$ \\
2) Denaturation & $96^{\circ} \mathrm{C}$ & $20 \mathrm{sec}$ \\
3) Annealing & $55^{\circ} \mathrm{C}$ & $30 \mathrm{sec}$ \\
4) Elongation & $60^{\circ} \mathrm{C}$ & $4 \mathrm{~min}$ \\
& \multicolumn{2}{r}{ Repeat steps 2-4 26 times } \\
5) Final elongation & $12^{\circ} \mathrm{C}$ & Hold \\
\hline
\end{tabular}

\subsubsection{Extraction of genomic DNA from flies}

30 male flies were collected in one eppendorf tube and snap frozen in liquid nitrogen. $400 \mu \mathrm{l}$ of lysis buffer containing $100 \mathrm{mM}$ TrisHCl, pH 7,5, $100 \mathrm{mM}$ EDTA, pH 8,0, $100 \mathrm{mM} \mathrm{NaCl}$ and 0,5\% SDS was added into the tube. Frozen flies were homogenized with a biovortexer. The lysate was incubated at $65^{\circ} \mathrm{C}$ for $15-30 \mathrm{~min}$. Afterwards, $228,4 \mu \mathrm{l}$ of $5 \mathrm{M} \mathrm{KAc}$ and $571,6 \mu \mathrm{l}$ of $6 \mathrm{M} \mathrm{LiCl}$ were added into the lysate and incubated on ice for $15 \mathrm{~min}$ before being centrifuged at 13,000 rpm for $15 \mathrm{~min}$. $1 \mathrm{ml}$ of supernatant was transferred into a new tube. $600 \mu \mathrm{l}$ of isopropanol was added and the mixture was centrifuged again at $13000 \mathrm{rpm}$ for $15 \mathrm{~min}$. DNA pellet was washed with $70 \%$ ethanol and dissolved in $150 \mu \mathrm{l}$ of sterile water.

\subsubsection{Extraction of genomic DNA from single flies}

For genotyping a large amount of fly stocks, DNA was isolated from single flies. Briefly, one male fly was snap-frozen in liquid nitrogen. Subsequently, the fly was squished in $50 \mu \mathrm{l}$ squishing buffer (10 mM TrisHCl pH 8,2, 1 mM EDTA, 25 mM NaCl) supplemented with $0,5 \mu$ l Proteinase $\mathrm{K}(20 \mathrm{mg} / \mathrm{ml})$ (Fermentas) using a pipette tip. The mixture was incubated for $20-30 \mathrm{~min}$ at $37^{\circ} \mathrm{C}$. Afterwards, Proteinase $\mathrm{K}$ was inactivated by incubation at $95{ }^{\circ} \mathrm{C}$ for $5 \mathrm{~min}$. The debris was pelleted and the supernatant could be directly used for PCR (2.2.1).

\subsubsection{Long-template PCR}

Expand Long Template PCR System (Roche) was used for the characterization of gene deletions in otk and otk2 mutant alleles. Components of PCR reaction and thermal cycles were set up according to the instructions. The PCR reaction mix used to amplify otk and otk2 genomic regions and the corresponding PCR program for long template PCR are shown in Table 14 and Table 15. 
Table 14: Long-template PCR.

\begin{tabular}{llll}
\hline & Buffer 1 & Buffer 2 & Buffer 3 \\
\hline Genomic DNA & $3 \mu \mathrm{l}$ & $3 \mu \mathrm{l}$ & $3 \mu \mathrm{l}$ \\
Forward Primer $(10 \mu \mathrm{M})$ & $2 \mu \mathrm{l}$ & $2 \mu \mathrm{l}$ & $2 \mu \mathrm{l}$ \\
Reverse Primer $(10 \mu \mathrm{M})$ & $2 \mu \mathrm{l}$ & $2 \mu \mathrm{l}$ & $2 \mu \mathrm{l}$ \\
dNTPs $(25 \mathrm{mM}$ each) & $0,7 \mu \mathrm{l}$ & $1 \mu \mathrm{l}$ & $1 \mu \mathrm{l}$ \\
10X buffer & $5 \mu \mathrm{l}$ & $5 \mu \mathrm{l}$ & $5 \mu \mathrm{l}$ \\
Enzyme mix & $0,75 \mu \mathrm{l}$ & $0,75 \mu \mathrm{l}$ & $0,75 \mu \mathrm{l}$ \\
ddH2O & ad $50 \mu \mathrm{l}$ & ad $50 \mu \mathrm{l}$ & ad $50 \mu \mathrm{l}$ \\
\hline
\end{tabular}

Table 15: PCR program for long-template PCR.

\begin{tabular}{lll}
\hline Step & Temperature & Duration \\
\hline 1) Initial denaturation & $93^{\circ} \mathrm{C}$ & $2 \mathrm{~min}$ \\
2) Denaturation & $93^{\circ} \mathrm{C}$ & $10 \mathrm{sec}$ \\
3) Annealing & $60^{\circ} \mathrm{C}$ & $30 \mathrm{sec}$ \\
4) Elongation & $68^{\circ} \mathrm{C}$ & $4-8$ min depending on length of expected fragment \\
& & Repeat steps 2-4 10 times \\
5) Denaturation & $93^{\circ} \mathrm{C}$ & $15 \mathrm{sec}$ \\
6) Annealing & $60^{\circ} \mathrm{C}$ & $30 \mathrm{sec}$ \\
7) Elongation & $68^{\circ} \mathrm{C}$ & $4-8 \mathrm{~min}+20$ sec for each cycle \\
& & Repeat steps 5-7 10 times \\
8) Final elongation & $68^{\circ} \mathrm{C}$ & 7 min \\
9) & $4^{\circ} \mathrm{C}$ & Hold \\
\hline
\end{tabular}

\subsection{Biochemical methods}

\subsubsection{Culture and transfection of Schneider S2R+ cells}

Schneider S2R+ cells, an immortalized culture of Drosophila embryonic cells, which expresses Frizzled2 (Schneider, 1972), were used for experiments in the cell culture system in this study. The cells were grown at $25{ }^{\circ} \mathrm{C}$ in Drosophila S2 medium (Invitrogen) supplemented with serum and antibiotics. Cells were counted using a Neubauer improved counting chamber. FuGene HD Transfection Reagent (Roche, Indianapolis, IN) was used for cell transfection according to the instructions. $2^{*} 10^{6}$ $\mathrm{S} 2 \mathrm{r}+$ cells were resuspended in $2 \mathrm{ml}$ fresh S2 medium with supplements. Resuspended cells were seeded in one well of a 6-well plate. $2 \mu \mathrm{g}$ of target plasmid was diluted in $100 \mu \mathrm{l}$ of sterile water. $4 \mu \mathrm{l}$ of FuGene transfection reagent was added 
into the plasmid solution and vortexed for $2 \mathrm{sec}$. The mixture was incubated at room temperature for $15 \mathrm{~min}$ before pipetting into the cell culture.

\subsubsection{Co-Immunoprecipitation (CoIP) in S2R+ cells}

For Co-Immunoprecipitation (CoIP), two wells of a 6-well plate were were transfected. The cells were propagated to T25 or T75 flasks after two days and harvested after another 2-3 days. Cells were harvested by scraping, pelleted by centrifugation at $1000 \mathrm{~g}$ for $5 \mathrm{~min}$ and washed two times with PBS. Cell lysis was done in $1 \mathrm{ml}$ freshly prepared cold CoIP buffer (50 mM TrisHCl pH7,5, $150 \mathrm{mM} \mathrm{NaCl}$, $1 \%$ NP40 with protease inhibitors (Pefabloc $200 \mu \mathrm{g} / \mathrm{ml}$, Pepstatin $2 \mu \mathrm{g} / \mathrm{ml}$, Aprotinin $2 \mu \mathrm{g} / \mathrm{ml}$, Leupeptin $2 \mu \mathrm{g} / \mathrm{ml}$ (Roche)) by homogenization using a $26 \mathrm{G}$ insulin syringe. Subsequently, the cells were disrupted by sonication with alternating bursts for $10 \mathrm{~min}$. The lysates were then centrifuged at $13000 \mathrm{rpm}$ for $15 \mathrm{~min}\left(4^{\circ} \mathrm{C}\right)$ to pellet the cell debris and the supernatant was transferred into fresh tubes and pre-cleared with $20 \mu \mathrm{l}$ of plain ProteinA/G Sepharose beads (BioCat) for $1 \mathrm{~h}$ on a rotator at $4^{\circ} \mathrm{C}$. At the same time, $30 \mu$ Protein A/G sepharose beads per sample in $300 \mu$ lysis buffer were combined with $200 \mu \mathrm{l}$ antibody (anti-Myc and anti- $\beta$-gal, see Table 6) and incubated for $1 \mathrm{~h}$ on a rotator at $4^{\circ} \mathrm{C}$. After pre-clearing, $20 \mu \mathrm{l}$ of each sample were kept as an input control and the rest was divided among the antibody-sepharose beads complexes. The antibody-antigen reaction took place for overnight on a rotator at $4^{\circ} \mathrm{C}$. The beads were subsequently washed 5 times with $800 \mu$ l CoIP buffer (centrifugation at $3000 \mathrm{rpm}$, for $2 \mathrm{~min}$ each time, $4^{\circ} \mathrm{C}$ ). $30 \mu \mathrm{l}$ of 2X SDS buffer (Wodarz, 2008) were added to each sample followed by incubation at $95^{\circ} \mathrm{C}$ for $5 \mathrm{~min}$ for protein denaturation. The samples were stored at $-20^{\circ} \mathrm{C}$ or used directly for SDS-PAGE and Western blot.

\subsubsection{Protein extraction from embryos}

Protein extraction under denaturing and non-denaturing conditions was performed according to standard procedures (Wodarz, 2008). Proteinase inhibitors were freshly added to the lysis buffer before use (see 2.3.2).

\subsubsection{Determination of protein concentration}

Protein concentration was determined according to Bradford method with Roti-

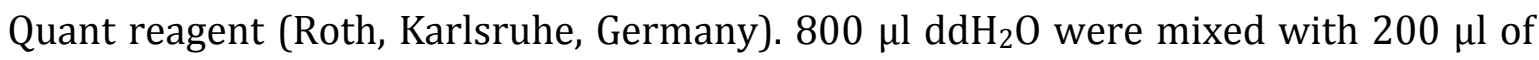


the reagent and $2 \mu \mathrm{l}$ of the protein lysate was added. $2 \mu$ lysis buffer were used as blank. The absorption was measured at $600 \mathrm{~nm}$ with a photometer. As a rule of thumb, OD600 =1 corresponds to approximately $1 \mathrm{mg} / \mathrm{ml}$ of total protein.

\subsubsection{SDS-polyacrylamide gel electrophoresis}

Discontinuous SDS-polyacrylamide gel electrophoresis (PAGE) allows the electrophoretic separation of proteins according to their size (Weber \& Osborn, 1969). SDS-PAGE was performed as described in (Wodarz, 2008). PageRuler Prestained Protein Ladder (Fermentas) was loaded to determine the size of the separated proteins.

\subsubsection{Western Blot}

Proteins separated by SDS-PAGE (2.3.5) were transferred onto a nitrocellulose membrane by wet blotting according to standard procedures (Wodarz, 2008). Primary and secondary antibodies used for Western blot are listed in Table 6 and Table 7. If the membrane was to be incubated with another primary antibody derived from another species than the first antibody, the HRP on the membrane was inactivated by incubation with $0,01 \%$ sodium azide in blocking buffer.

\subsubsection{Coomassie staining}

Polyacrylamide gels were rinsed with $\mathrm{ddH}_{2} \mathrm{O}$ and stained with Brilliant Blue $\mathrm{R}$ Concentrate (Sigma Aldrich) diluted 1:5 for 30-60 $\mathrm{min}$ at room temperature. Afterwards, the gel was rinsed with $\mathrm{ddH}_{2} \mathrm{O}$ and destained in $10 \% \mathrm{v} / \mathrm{v}$ acetic acid and $20 \% \mathrm{v} / \mathrm{v}$ ethanol. Brilliant Blue R concentrate is a methanol based stain. The stain contains methanol and acetic acid so gels do not require fixing prior to staining.

\subsubsection{GST fusion protein purification for antibody generation}

$100 \mathrm{ml}$ of 2 xYTA medium (see 2.1.3) was inoculated with $5 \mathrm{ml}$ overnight culture of BL21 bacteria transformed with OtkEx-pGEX4T1 and incubated at $37{ }^{\circ} \mathrm{C}$ until the culture reached high density $(O D 600=2) .2 \mathrm{ml}$ ethanol was added to induce chaperones. IPTG was added to a final concentration of $0,1 \mathrm{mM}$ to induce the expression of recombinant protein. After incubation for $3-5 \mathrm{~h}$ at $20^{\circ} \mathrm{C}$ bacteria were harvested by centrifugation at $7700 \mathrm{~g}$ for $10 \mathrm{~min}$ at $4^{\circ} \mathrm{C}$. The pellet was resuspended 
in $5 \mathrm{ml}$ lysis buffer (50 mM TrisHCl pH7,5, $150 \mathrm{mM} \mathrm{NaCl}, 1 \mathrm{mM}$ EDTA) and frozen at $80^{\circ} \mathrm{C}$ overnight to disrupt the bacteria.

The pellet was thawed in warm water and immediately proteinase inhibitors (see 2.3.3), $1 \mathrm{mM}$ DTT and $1 \mathrm{mg} / \mathrm{ml}$ lysozyme were added. Bacteria were disintegrated by sonication with $10 \mathrm{sec}$ bursts alternated with $10 \mathrm{sec}$ of incubation on ice. $10 \%$ TritonX-100 was added to the lysate to a final concentration of $1 \%$. The mixture was gently rotated for 30-60 min and ultra-centrifuged at $100000 \mathrm{~g}$ for $1 \mathrm{~h}$ at $4^{\circ} \mathrm{C} .100 \mu \mathrm{l}$ of 50:50 slurry of Glutathione-Sepharose beads (GE Healthcare) was added to the supernatant and rotated for $30 \mathrm{~min}$ at room temperature. The beads were sedimented and washed three times with PBS. The bound protein was eluted from the beads by elution buffer containing $15 \mathrm{mM}$ reduced glutathione in $50 \mathrm{mM}$ TrisHCl, $\mathrm{pH} 8,0$. Eluted protein was snap frozen and stored at $-80^{\circ} \mathrm{C}$ for later use.

Protein concentration was estimated by running the samples on a SDS gel together with standard samples containing known concentrations of BSA and subsequent Coomassie staining (2.3.7).

\subsection{Immunohistochemistry}

\subsubsection{Fixation and immunofluorescent staining of embryos}

Formaldehyde fixation and incubation of fixed embryos with primary and secondary antibodies was done according to standard procedures (Müller, 2008).

\subsubsection{Fixation and immunofluorescent staining of larval tissues}

Larval brains of wandering third instar larvae were dissected in PBS and then fixed in 4\% formaldehyde in PBS for $20 \mathrm{~min}$ at room temperature on a rocking platform. The tissue was washed three times with PBTw (PBS, 0,1\% Tween) and thereafter permeabilized by treating with PBS containing $1 \%$ Triton X-100 for $1 \mathrm{~h}$ at room temperature on a rocking platform. Subsequent steps were performed according to the protocol for antibody staining of embryos (2.4.1).

Imaginal discs were dissected in cold PBS and fixed in 4\% formaldehyde in PBS for $20 \mathrm{~min}$ at room temperature on a rocking platform. Subsequent steps were performed according to the protocol for antibody staining of embryos (2.4.1). 


\subsubsection{Fixation and immunofluorescent staining of adult tissues}

Adult testes were dissected in a drop of PBS and fixed in $4 \%$ formaldehyde in PBS for $20 \mathrm{~min}$ at room temperature on a rocking platform. Subsequent steps were performed according to the protocol for antibody staining of embryos (2.4.1). Actin was stained with Phalloidin Alexa Fluor 555 diluted 1:100 (Molecular Probes, A34055) together with the secondary antibodies.

\subsubsection{Fluorescent in situ hybridization (FISH) of embryos}

FISH was performed using the Tyramide Signal Amplification (TSA) Kit (Molecular Probes) according to the protocol provided. Fixation of embryos was performed as described in 2.4.1. For preparation of a Digoxigenin (DIG)-labelled RNA probe, $5 \mu \mathrm{g}$ of template DNA was linearized in $30 \mu \mathrm{l}$ final volume. The linearized template was then purified using the High Pure PCR Product Purification Kit (Roche), according to manufacturer's instructions except for the elution volume which was adjusted to $20 \mu$ l. In vitro transcription and incorporation of DIG was performed with the DIG RNA labeling kit (SP6/T7) (Roche) according to manufacturer's instructions. The labeled probe was purified using the RNeasy ${ }^{\circledR}$ Plus Mini Kit (Qiagen). Fragmenting of the probe was performed according to the instructions provided in the TSA Kit (Molecular Probes). The purified DIG labeled probe was mixed with hybridization solution to a final volume of $1,5 \mathrm{ml}$. At this point the probe could be stored at $-20^{\circ} \mathrm{C}$.

\subsubsection{Mounting of adult wings}

Wings were removed from adult flies and dehydrated in $100 \%$ isopropanol for at least $5 \mathrm{~min}$. Wings were placed on a glass slide and the isopropanol was allowed to evaporate. A small drop of Roti® Histokitt (Roth) was put onto the wing and they were covered with a cover slip for microscopic analysis.

\subsubsection{Microscopy and image acquisition}

Samples were examined using 25 X 0,8 NA Zeiss Plan-Neofluar and 63 X 1,4 NA Zeiss Plan-Apochromat oil immersion objectives on a confocal laser-scanning microscope (Carl Zeiss LSM 510 Meta). Pinholes were set to 1 airy unit for image acquisition. Images were captured by 1024 x 1024 pixels at the appropriate zoom using 2-line mean averaging. Brightfield images were acquired with an AxioImager.Z1 upright 
microscope using 10 X 0,3 NA Zeiss Plan-Neofluar and 25 X 0,8 NA Zeiss PlanNeofluar oil immersion objectives.

\subsection{Genetic methods}

\subsubsection{Fly breeding and fly stocks}

The fly stocks used in this work were kept at $18^{\circ} \mathrm{C}, 25^{\circ} \mathrm{C}$ or $29^{\circ} \mathrm{C}$ on standard medium (Ashburner, 2004). For collection of embryos flies were kept in egg collection cages on apple juice agar plates. To stimulate egg-laying, a small amount of yeast paste was applied at the centre of the apple juice plate.

Standard medium: 712 g cornmeal, 95 g soya flour, 168 g dry yeast, 450 g malt extract, $150 \mathrm{ml}$ 10\% Nipagin $(700 \mathrm{ml}$ 99\% ethanol, $300 \mathrm{ml} \mathrm{H} 2 \mathrm{O}, 100 \mathrm{~g}$ Nipagin ( $\left.\mathrm{C}_{8} \mathrm{H}_{8} \mathrm{O}_{3}\right)$ ), $45 \mathrm{ml}$ propionic acid, $50 \mathrm{~g}$ agar, $400 \mathrm{~g}$ sugar beet sirup, add 9,75 $\mathrm{lddH}_{2} \mathrm{O}$

Apple juice plates: $40 \mathrm{~g}$ agar, $1 \mathrm{l} \mathrm{H}_{2} \mathrm{O}, 340 \mathrm{ml}$ apple juice, $17 \mathrm{~g}$ sugar, $20 \mathrm{ml} 10 \%$ Nipagin

\subsubsection{UAS-GAL4 system}

The UAS-GAL4 system (Brand \& Perrimon, 1993) is used to ectopically overexpress the gene of interest in a time and tissue specific manner. The method makes use of the yeast transcription factor GAL4 that binds to an upstream activating sequence (UAS), thereby activating gene expression. In UAS reporter flies the gene of interest is placed under the control of UAS. Another fly stock, the so-called GAL4 driver line, contains the GAL4 gene under the control of a promoter or enhancer of a gene with a known expression pattern. When the two fly stocks are crossed, GAL4 activates the expression of the gene of interest. By choosing different GAL4 driver lines, the gene can be expressed in a wide variety of tissues and developmental stages. GAL4 driver lines and UAS reporter flies used in this work are described in Table 8.

\subsubsection{Generation of transgenic flies with white- background}

Different constructs inserted in P-element based vectors were used to generate transgenic flies. The vectors were injected into the posterior end of syncytial 
blastoderm white- embryos together with another plasmid encoding transposase. This transposase activity mediates integration of the transgenic construct into the genomic DNA (Rubin \& Spradling, 1982). Subsequently, transgenic flies that have successfully integrated the P-element could be recognized by red eye color, since the vectors used for injection contain the mini-white gene (Bachmann \& Knust, 2008). Briefly, $20 \mu \mathrm{g}$ of plasmid was mixed with $5 \mu \mathrm{g}$ of transposase DNA in $50 \mu \mathrm{l}$ injection buffer containing $5 \mathrm{mM} \mathrm{KCl}, 0.1 \mathrm{mM}$ sodium phosphate, $\mathrm{pH}$ 6,8. white- flies were allowed to lay embryos on apple juice plates for $20 \mathrm{~min}$ at $18^{\circ} \mathrm{C}$. The embryos were dechorionated, aligned on cover slips and immersed in 10S Voltalef oil (Prolabo). The plasmid mixture was injected into the pole cells at the posterior ends of the embryos using a micromanipulator (InjectMan NI2, Eppendorf). After injection, embryos were kept in Voltalef oil at $18{ }^{\circ} \mathrm{C}$ for $48 \mathrm{~h}$ before the hatched larvae were collected. Flies were single-crossed to $w$-;Gla/CyO(ftz::lacZ) flies for the selection of positive transgene insertion and further insertion site analysis. The transgenic constructs used for injection are listed in Table 5.

\subsubsection{Generation of transgenic flies using ФC31-mediated integration}

The Streptomyces phage $\Phi C 31$ is able to insert its own genomic DNA into that of bacterial genomes using an enzyme called integrase. Unlike transposase, which mediates genome-wide P-element insertion, the ФC31 integrase operates in a sequence-specific and unidirectional manner. The P-element vectors contain both the transgene and a donor sequence (attB) and fly stocks used for injection have to carry an attP sequence which acts as the recipient site (Groth et al, 2004; Fish et al, 2007). Furthermore, the flies express ФC31 integrase under control of the vasa promoter, providing an endogenous source of ФC31 integrase (Bischof et al, 2007). This results in the site-specific insertion of the attBcontaining transgene into the attP site. Hybrid sites (attL and attR) are formed during this process and prevent further integrase-catalyzed movement of the integrated transgene (Fish et al, 2007) (Fig. 11). Fly stocks used for ФC31-mediated integration are listed in Table 8 and constructs used for injection in Table 5. 


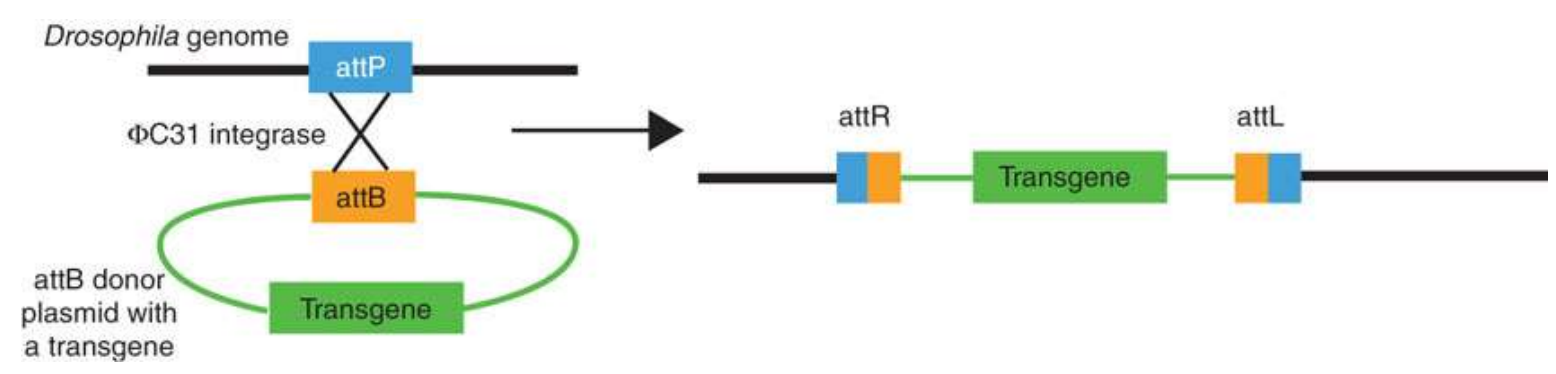

Fig. 11: Scheme of $\Phi C 31$ integrase-mediated trangene integration.

The preplaced attP sequence acts as the recipient site in the Drosophila genome. A P-element based plasmid contains both the transgene and a donor attB sequence and is injected into attP-containing recipient embryos. This results in site-specific insertion of the transgene into the attP site. The formation of hybrid attR and attL sites prevents further integrase-mediated movement of the already integrated transgene. Taken from (Fish et al, 2007).

\subsubsection{FLP-FRT mediated gene deletion}

To generate a deletions of otk and otk2 rush, FLP-FRT mediated gene deletion was employed (Parks et al, 2004). The method makes use of the ability of FLP recombinase to mediate recombination between two FRT sites located on homologous chromosomes (Golic \& Lindquist, 1989). By this, a deletion of a genomic region flanked by two transposons that contain FRT sites can be achieved. Upon induction of FLP recombinase the genomic region between two FRT sites in trans is deleted, leaving a single transposon at the deletion site. Successful recombination between the two FRT sites in trans can be detected by loss of red eye color, if white genes are deleted together with the genomic region or by PCR (Fig. 12).

A
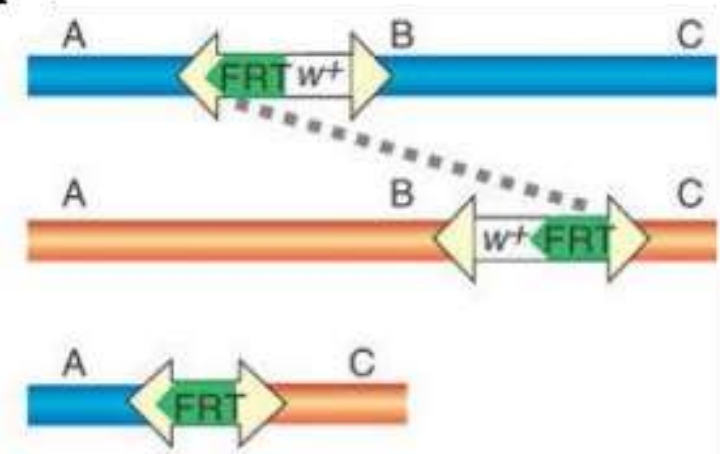

B
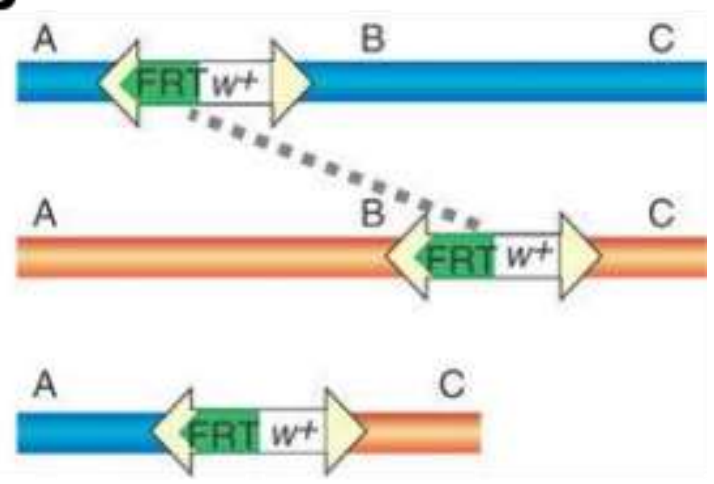

Fig. 12: Schematics of FLP-FRT recombination of two FRT containing transposons in trans.

The chromosomes are shown in orange and blue, the transposon insertion elements are indicated in light yellow and FRT elements in green. The order of theoretical markers A,B and C along the chromosome is indicated. FLP-FRT recombination allows the generation of white- deficiencies (A) or white+ deficiencies (B), depending on the orientation of the white+ containing P-element. Modified from (Parks et al, 2004) 
In case of otk and otk2, three suitable transposon insertion lines containing FRT sites were available from the Harvard stock collection. (Fig. 13A). The P(XP)d01360 element is located upstream of the 5'UTR of otk, while the piggyBac insertion line PBac(RB)e03992 element is inserted downstream of the 3'UTR of otk. Furthermore, the $\mathrm{PBac}(\mathrm{PB}) \mathrm{c01790}$ element is located upstream of otk2 in the second exon of mppe and P(XP)d01360 is downstream of the 3'UTR of otk2 (Fig. 13A). Like this it was possible to generate null alleles of otk, otk2 as well as a double knock-out of otk,otk2. The features of the $P(\mathrm{XP})$ and piggyBac lines have been described (Thibault et al, 2004) and are depicted in (Fig. 13B).

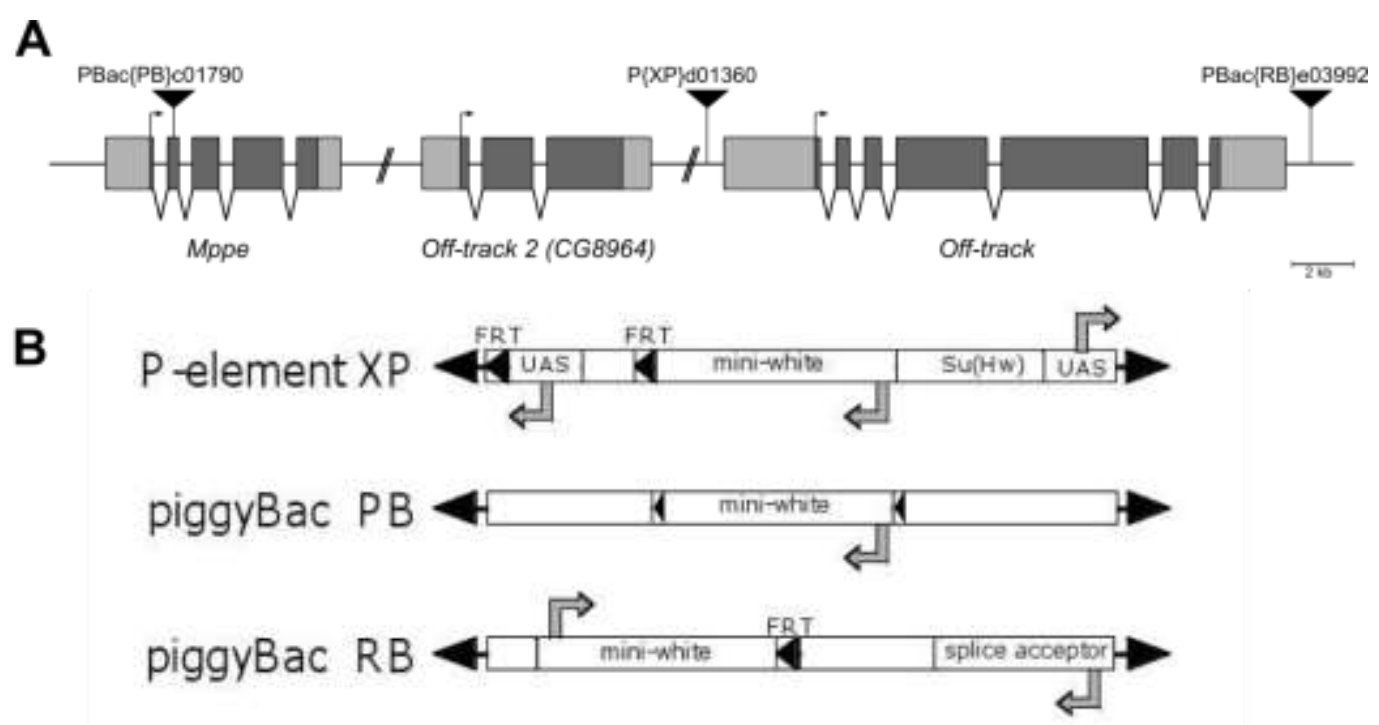

Fig. 13: P-elements used for FLP-FRT recombination mediated deletion of otk and otk2.

(A) Overview of the genomic region of otk, otk2 and the neighbouring gene Mppe on chromosome 2R. Null alleles for $o t k$ and $o t k 2$ alone as well as both $o t k$ and $o t k 2$ were generated via FLP/FRT mediated recombination between FRT sites contained in P(XP)d01360 and PBac(RB)e03992 (otk deletion), $\mathrm{PBac}(\mathrm{PB}) \mathrm{c} 01790$ and $\mathrm{P}(\mathrm{XP}) \mathrm{d} 01360$ (otk2 deletion) or PBac(PB)c01790 and PBac(RB)e03992 (otk,otk2 deletion). (B) Schmatic representations of the P-elements utilized. The piggyBac vector (PB) comprises a mini-white gene flanked by short (48 bp) FRT sites, whereas PBac(RB) and $\mathrm{P}(\mathrm{XP})$ contain long (199 bp) FRT sites. Modified from (Thibault et al, 2004).

Genomic maps and crossing schemes to generate otk and otk2 as well as otk,otk2 deletions are provided in the appendix. In brief, a heat-inducible FLP recombinase was introduced on the first chrosmosome and L2 larvae were heat-shocked for two hours at $37^{\circ} \mathrm{C}$ on two consecutive days. Mutant flies carrying the otk deletion were selected by the loss of the mini-white genes carried by both transposon lines and resulting in white eye colour. Flies carrying a potential deletion for otk2 were 
screened by PCR, since no selection based on eye-colour was possible. In case of the otk,otk2 double knock-out, both eye colour phenoptypes were possible, depending which FRT site would be used for recombination. Flies selected based on white eye phenotype were screened first.

\subsection{Viability and Life span measurements}

\subsubsection{Viability analysis}

Viability was determined by aligning about 100 embryos on apple juice agar plates. The embryos were allowed to develop at $25{ }^{\circ} \mathrm{C}$ and hatching rates were recorded after at least $24 \mathrm{~h}$.

\subsubsection{Life span analysis}

Survival curves were recorded as previously described (Wang et al, 2003). Cohorts of about 100 males or females were separated two days after hatching and were transferred to fresh vials. Subsequently, flies were transferred to fresh vials every four days and the number of dead flies was recorded.

\subsection{Phylogenetic analysis}

\subsubsection{Phylogenetic trees}

Phylogenetic analyses were conducted with Mega software version 5 (Tamura et al, 2011) using ClustalW alignment and neighbor-joining method with a gap opening penalty of 60 .

\subsubsection{Dot Plot Analysis}

Dot Plot analysis was performed using DottupP with word size 6 (http://srs.ebi.ac.uk/). 


\section{RESULTS}

\section{1 off track (otk) and the neighboring gene off-track2 (otk2, CG8964) are paralogs evolved by gene duplication}

The off-track (otk) gene is located on the right arm of the second chromosome (2R: 7,888,983 - 7,907,329(-) ) and codes for a protein with a length of 1033 amino acids. I performed a protein BLAST search with the protein sequence of Otk to identify potential additional homologs in the Drosophila genome. Indeed, I found that the protein encoded by the annotated and so far not described gene CG8964 is closely related to Otk (53\% identity over 427 amino acids, Fig. 14). CG8964 is located upstream and right next to otk on the second chromosome (2R: 7,910,651 $7,912,775(-))$, suggesting that it is the result of a gene duplication. Therefore, the gene CG8964 was named off-track2 (otk2).
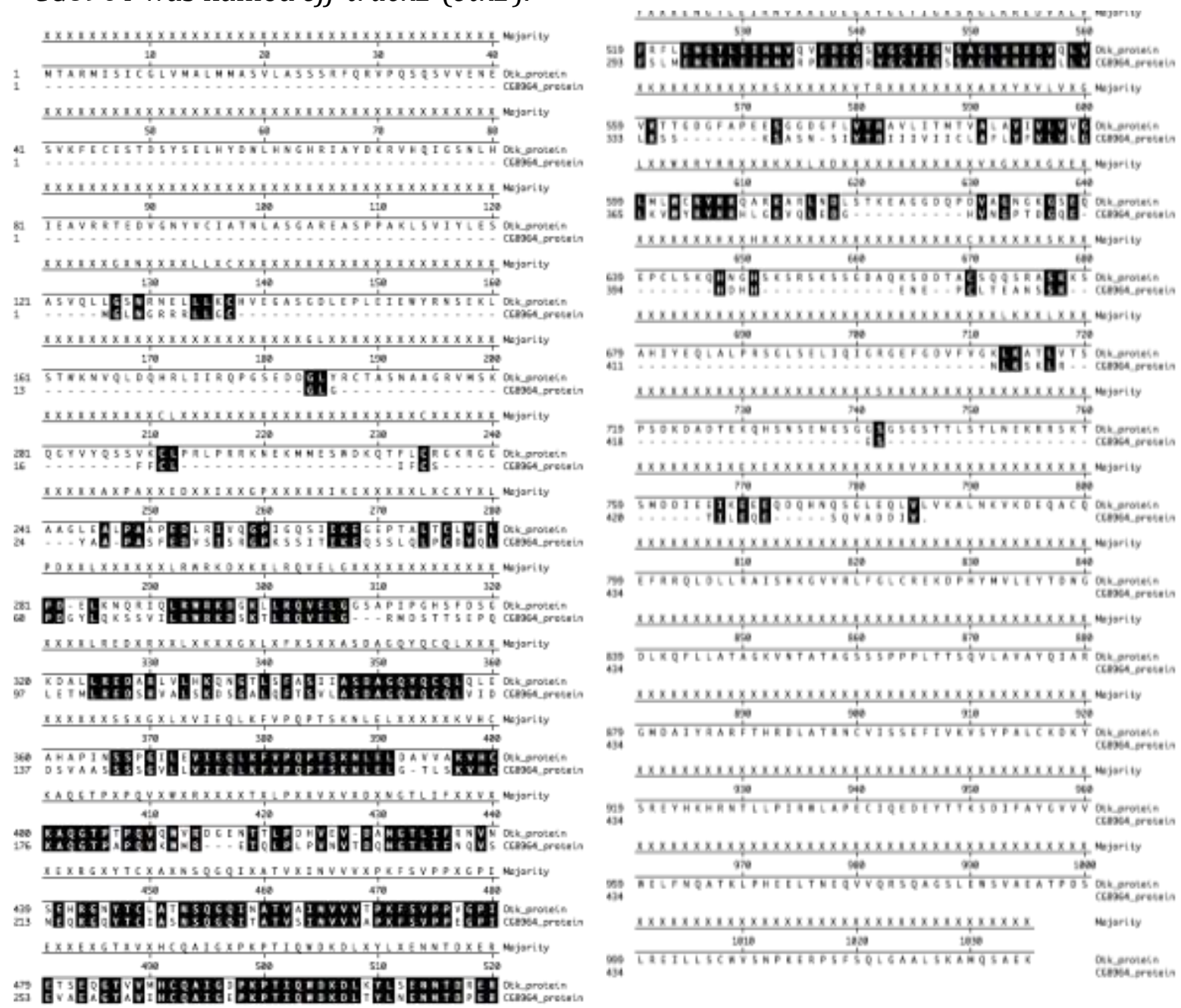

Fig. 14: Alignment of the protein sequences of Otk and the gene product of CG8964. 
Phylogenetic analysis confirmed that Drosophila otk and otk2 are indeed two paralogs of the single PTK7 gene in mouse and human (Fig. 15 A). To test whether the gene duplication is specific for Drosophila or occurred also in other arthropods, the sequences from different arthropod species homologous to Drosophila Otk and Otk2 were analyzed. The resulting phylogenetic tree clearly shows that two Otk paralogs can only be found in Drosophila species, but not in other arthropod species (Fig. 15 B). Hence, a lineage specific duplication has generated two PTK7 homologs in Drosophila.

Structurally, Otk is a transmembrane molecule. It consists of five extracellular immunoglobuline-like domains, a single transmembrane domain and a kinase homology domain on the C-terminus (Fig. 16 A). Its paralog Otk2 only comprises three immunoglobuline-like domains and only a very short stretch of 69 amino acids intracellularly (Fig. 16 A). To answer the question which parts of the much shorter Otk2 sequence correspond to which parts of the Otk sequence, a dot plot was performed, visualizing matching residues in both sequences. This analysis demonstrated that the entire 0tk2 sequence (except for the signal peptide) can be matched to a continuous stretch within the Otk sequence (Fig. 16 B), ranging from the third immunoglobulin-like domain until the beginning of the kinase homology domain. 


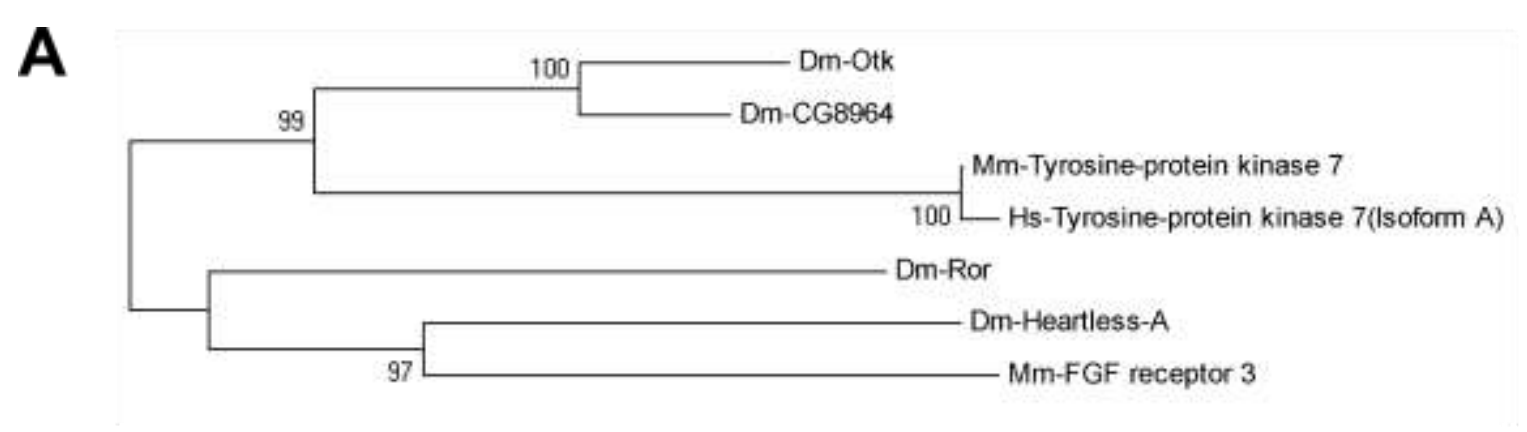

B

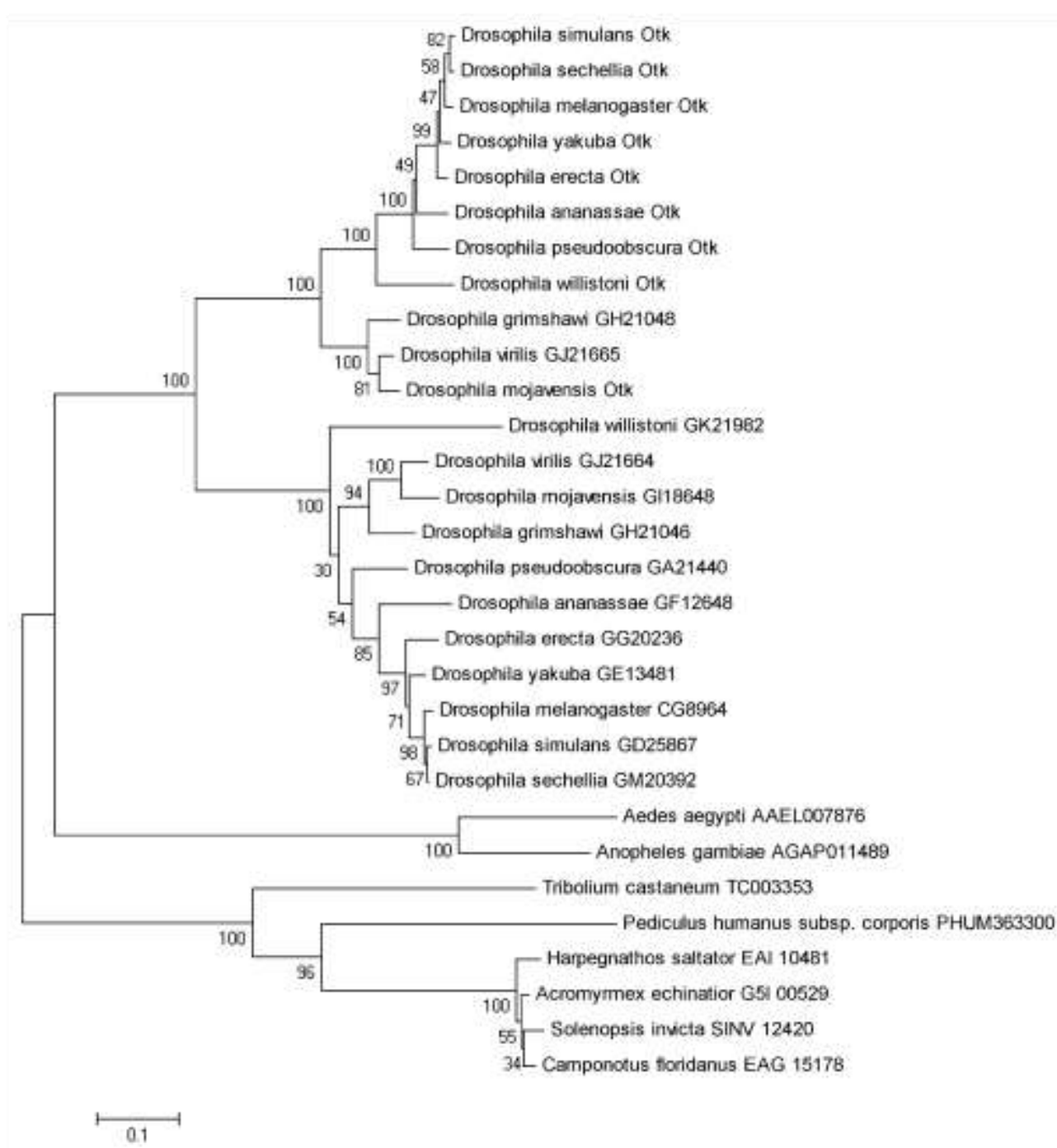

Fig. 15: Off-track (Otk) and Off-track2 (CG8964, Otk2) are paralogs evolved by gene duplication. (A) Phylogenetic tree representation of an alignment of sequences from different species homologous to Drosophila Otk. Blast-P of Dm-Otk in Drosophila returned Dm-CG8964 (Otk2) as the best hit; Dm-Ror and Dm-Heartless-A were the second and third hit, repectively. Blast-P of Dm-Otk in Mouse returned Mm-PTK7 as the best hit, while Mm-FGF receptor3 was the second best hit. Blast-P of Dm-Otk in Human returned Hs-PTK7 as the best hit. ClustalW alignment of these sequences and Neighbor-Joining tree confirms that the PTK7 branch is separated from the other proteins by a bootstrap value of 100. In this branch, two Drosophila genes, but only one in the other species can be found. Dm, Drosophila melanogaster; Mm, Mus musculus; Hs, Homo sapiens. (B) Phylogenetic tree of sequences from different arthropod species homologous to Drosophila Otk. 
A

Off-track
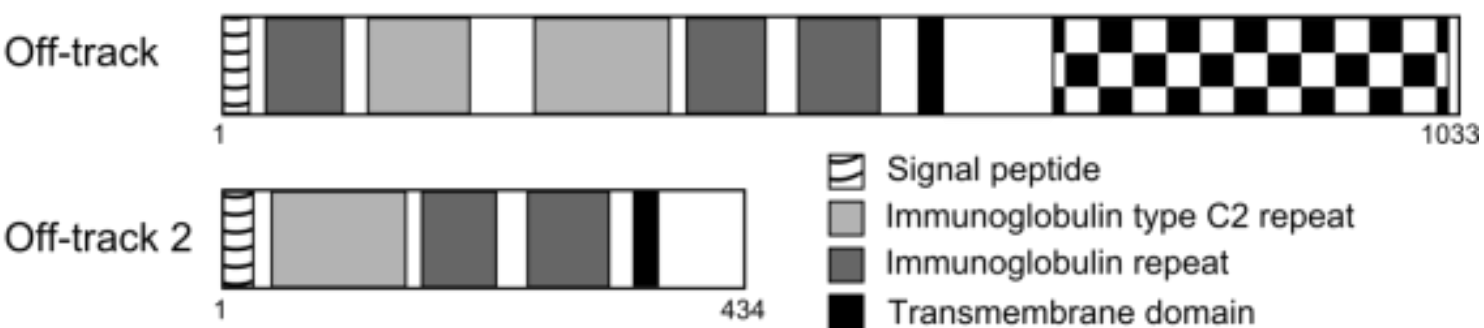

100 aa

Signal peptide

1033

$\square$ Immunoglobulin type $\mathrm{C} 2$ repeat

Immunoglobulin repeat

Transmembrane domain

Tyrosine kinase domain

B

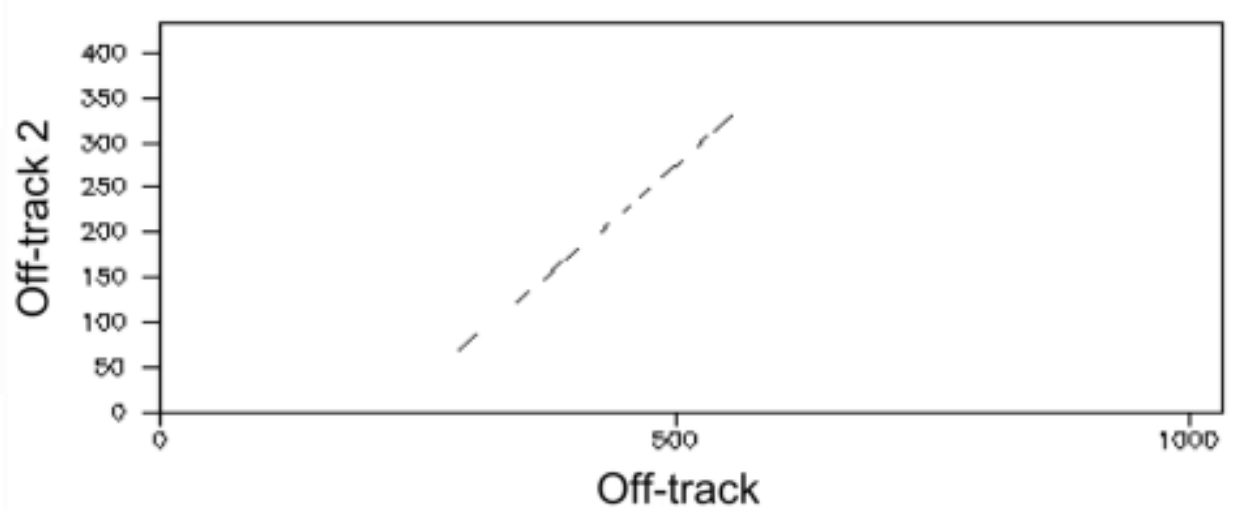

Fig. 16: Structural similarity between Otk and Otk2.

(A) Protein structures of Otk and Otk2. Domains were predicted using the SMART sequence analysis tool. (B) Dot plot comparing the protein sequences of Otk (x-axis) and Otk2 (y-axis) to visualize matching residues in both sequences.

As already mentioned, Otk2 lacks the entire tyrosine kinase homology domain. In other species it was shown for PTK7 that the tyrosine kinase domain does not possess any kinase activity (Chou \& Hayman, 1991; Mossie et al, 1995; Miller \& Steele, 2000; Jung et al, 2004). Interestingly, the short intracellular amino acid stretch of Otk2 (aa 366 - 434) contains a motif that is highly conserved between Otk2 and Otk (Fig. 17).

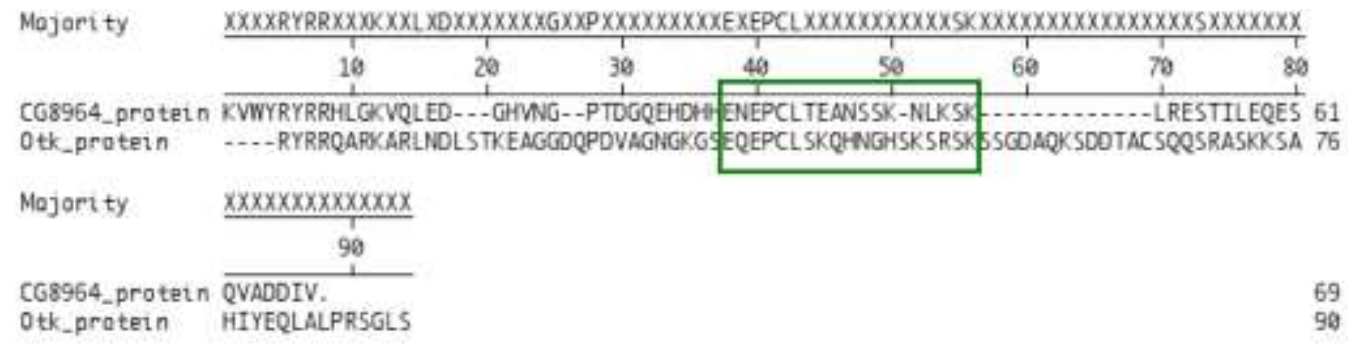

Fig. 17: Alignment of the intracellular carboxy terminus of 0tk2 with the corresponding 0tk sequence. 
The existence of two closely related PTK7 homologs could mean that otk and otk2 function redundantly and may explain why these genes have not been found in previous screens for planar cell polarity and Wnt signaling pathway phenotypes.

\subsection{Biochemical interactions of Off-track and Off-track2}

It is known for Xenopus PTK7 that the protein forms homodimers (Annette Borchers, personal communication). To test whether 0tk and 0tk2 also have the ability to interact with themselves or possibly also with each other, I performed immunoprecipitation experiments with transiently transfected S2r+ cells.

To test for homodimerization, Myc- and GFP-tagged Otk constructs under the control of an Actin promoter were co-overexpressed in S2r+ cells. Cell lysates were immunoprecipitated using anti-Myc antibody. Immunoblotting with anti-GFP antibody demonstrated that Otk-GFP could be co-precipitated with Otk-Myc. The same experiment was performed with Myc- and GFP-tagged Otk2 constructs and showed that Otk2-GFP co-immunoprecipitates with Otk2-Myc as well. As control, cells were transfected with GFP-tagged constructs only and with an empty vector (Fig. 18 A). Hence, both Otk and Otk2 appear to be able to form homodimers, as described for the vertebrate homologs.

The existence of the Otk paralog Otk2 in Drosophila raises the question whether the two proteins can also interact with each other. To test for heterodimerization, Myctagged Otk2 and GFP-tagged Otk constructs under the control of an Actin promoter were co-overexpressed in S2r+ cells. Cell lysates were subjected to anti-Myc IP. Immunoblotting with anti-GFP antibody demonstrated that Otk-GFP could be coprecipitated with Otk2-Myc. The reverse experiment was performed with Myc-tagged Otk and GFP-tagged Otk2 constructs and showed that Otk2-GFP coimmunprecipitates with Otk-Myc as well (Fig. $18 \mathrm{~B}$ ). From these results it can be concluded that Otk and Otk2 most likely can form both homo- and heterodimers, however, it remains to be investigated whether other proteins are involved in this interaction. 


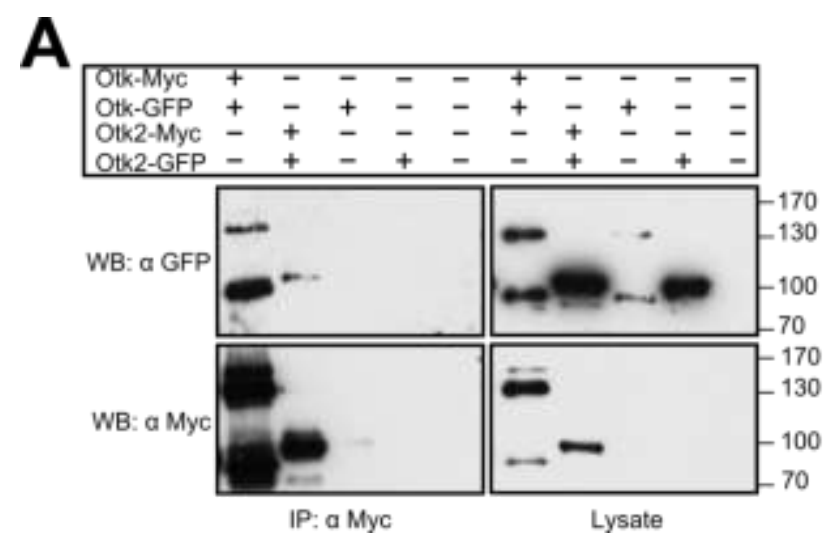

B

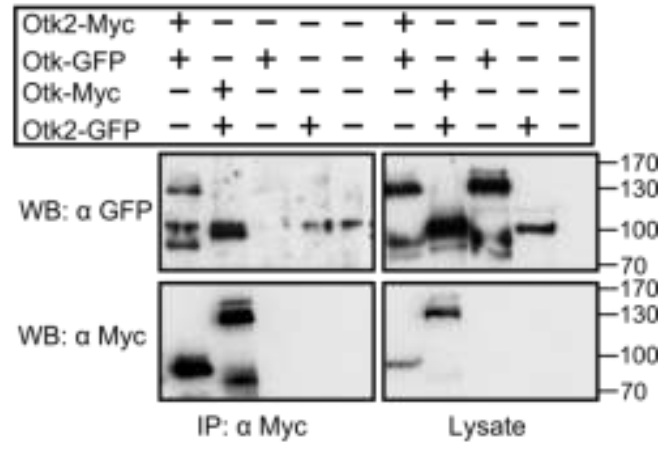

Fig. 18: Off-track and Off-track2 form homodimers and heterodimers.

(A and B) Otk-myc or Otk2-myc and Otk-GFP or Otk2-GFP expression vectors were transfected as indicated in Drosophila S2r+ cells. Cell lysates were immunoprecipitated and analyzed by Western Blot with the indicated antibodies. IP, Immunoprecipitation; WB, Western Blot.

As the previous results do not provide any information about the domains involved in the dimerization, a first approach using Otk deletion constructs was undertaken to further narrow down the regions required for homo- and heterodimerization. S2r+ cells were transiently transfected with Myc-tagged full length Otk or two deletion constructs of Otk in combination with GFP-tagged Otk or Otk2 and were subjected to anti-Myc IP with subsequent immunoblot against GFP. Both a version of Otk lacking the extracellular domain and a C-terminally truncated Otk protein were able to coimmunoprecipitate full length Otk as well as Otk2 (Fig. 19). This result suggests that the transmembrane domains could mediate their interaction. 


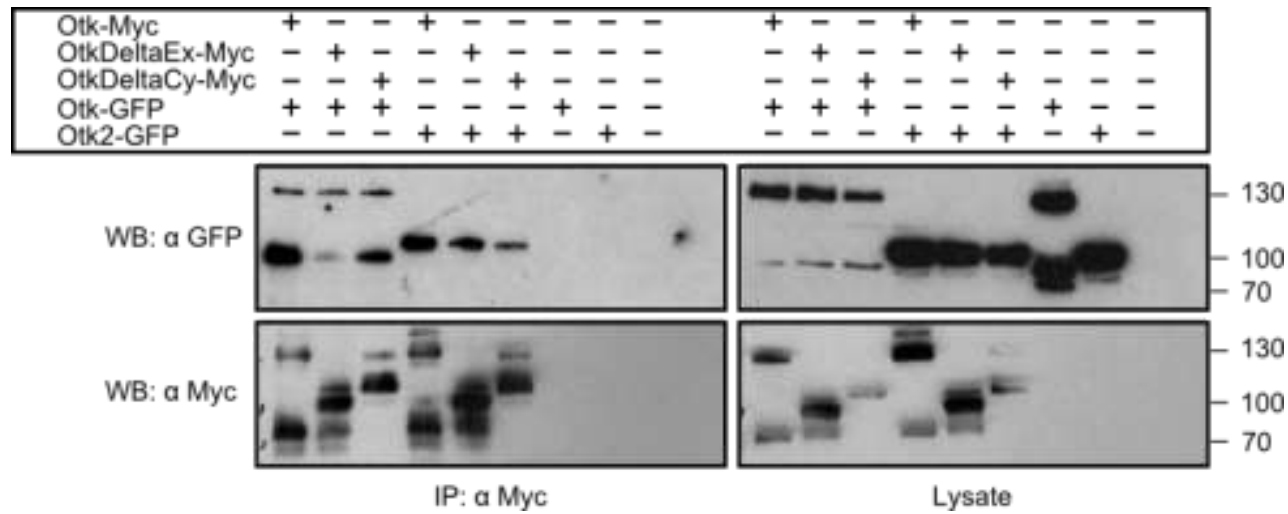

Fig. 19: Homo- and heterodimerization of Otk and 0tk2 requires the transmembrane domain. Otk-myc or myc-tagged Otk deletion contructs and Otk-GFP or Otk2-GFP expression vectors were transfected as indicated in Drosophila S2r+ cells. Cell lysates were immunoprecipitated and analyzed by Western Blot with the indicated antibodies. OtkDeltaCy lacks the cytoplasmic domain (aa 7761033) and OtkDeltaEx lacks the Extracellular domain (aa 2-474). IP, Immunoprecipitation; WB, Western Blot.

\subsection{Expression of Off-track and Off-track2}

Otk has been described 20 years ago to be dynamically expressed during Drosophila embryogenesis (Pulido et al, 1992). To verify the expression pattern of Otk and to thereby gain further insight into the potential function of the two proteins, several approaches were undertaken.

\subsubsection{Expression of Off-track and Off-track 2 reporter lines}

A homozygous viable GFP enhancer trap line in the otk locus and a lacZ enhancer trap

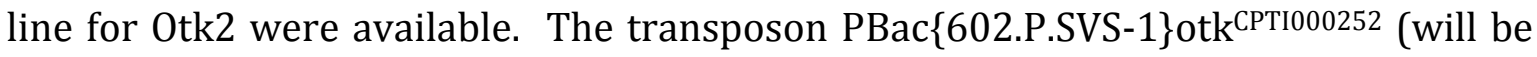
called otk ${ }^{C P T 1000252}$ for simplification) is inserted in the first intron of the otk locus (position 2R:7,902,551 (+)) and encodes for a GFP gene trap. The line is not a functional protein trap and thus does not provide any insight into the subcellular localization, but is still useful as a reporter line. GFP expression could be observed in a segmental pattern during early embryonic development from stage 9 on (Fig. $20 \mathrm{~A}$, B). It is possible that expression starts earlier, but is too weak to be detected. Later on, expression could be observed in the developing nervous system and in the developing gut (Fig. 20 C-F). Dissection of wandering L3 larvae revealed expression in the leg imaginal disc (Fig. $20 \mathrm{G}$ ) as well as in the brain (Fig. $20 \mathrm{H}-$ J). No expression was observed in wing imaginal discs (data not shown), which was surprising regarding a 
predicted function of Otk in planar cell polarity signaling. In the brain, strong reporter expression was seen in the optic lobes of the central nervous system (Fig. $20 \mathrm{H}$ ). Coimmunostaining with neuroblast markers showed that the Otk reporter is not expressed in neuroblasts but in the surrounding cells, which are most likely neuroblast progeny (Fig. 20 I). Furthermore, expression could be observed in a dorsal structure in the brain, which possibly is the developing mushroom body.

The transposon P\{lacW\}l(2)SH16395H1639 (will be called CG8964 ${ }^{\text {SH1639 }}$ for simplification) is inserted in the 5'UTR of the CG8964 locus (position 2R:7,912,740..7,912,810) and encodes for a lacZ enhancer trap. LacZ expression could be detected in segmental stripes in early embryonic development and later on in the developing gut as well as the developing nervous system (Fig. 20). Hence, the expression is very similar to that observed for otk ${ }^{C P T 1000252}$, indicating that the two proteins play a role in the same processes. 

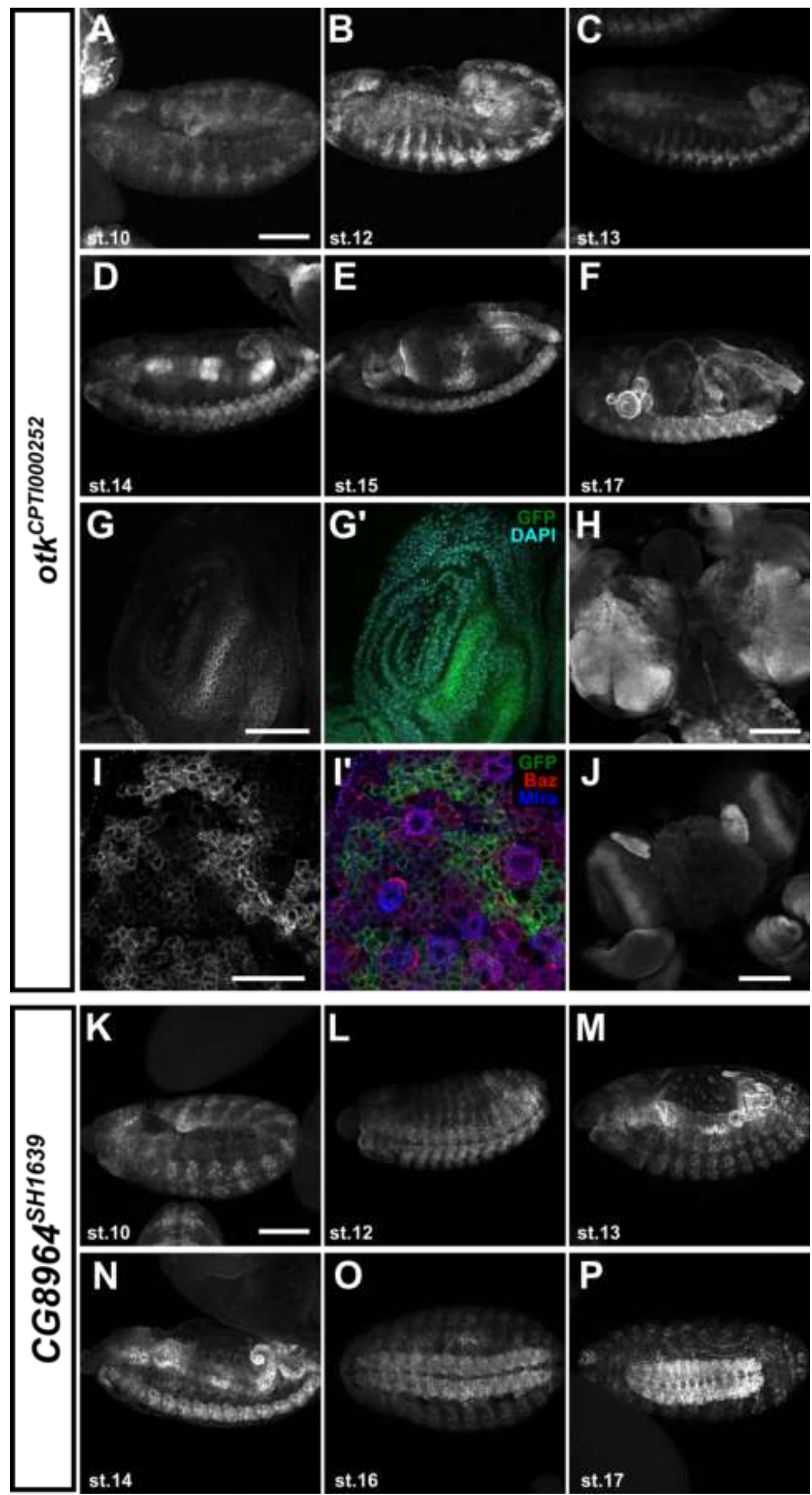

Fig. 20: Expression of Off-track and Off-track2 reporter lines. 
Fig. 20 (previous page): (A-F) Transgenic embryos expressing a GFP gene trap in the otk locus were stained against GFP. Stages of embryonic development are indicated. (G) Leg imaginal disc from L3 larvae expressing otk GFP gene trap stained against GFP. (H-J) Brain from L3 larvae expressing otk GFP gene trap stained against GFP. (H) Dorsal view of the brain. (I) Magnification of the optic lobes. Miranda (Mira) and Bazooka (Baz) were co-stained to mark the neuroblasts. (J) Ventral view of the brain. (K-P) Transgenic embryos expressing a lacZ gene trap in the otk2 locus were stained against beta-galactosidase. Stages of embryonic development are indicated. Anterior is to the left in A-F and KP. Scale bars: A-F, H, J, K-P = $100 \mu \mathrm{m}, \mathrm{G}=50 \mu \mathrm{m}, \mathrm{I}=20 \mu \mathrm{m}$.

\subsection{2 mRNA Expression of Off-track and Off-track2 during embryonic development}

To confirm the results obtained from the analysis of the otk and otk2 reporter lines and to gain further insight into the mRNA localization, fluorescent in situ hybridzation (FISH) was performed. Both Otk and Otk2 mRNA are expressed in segmental stripes in the process of germband extension (Fig. 21 A; Fig. 22A). During germband retraction, both mRNAs start to be expressed in the developing nervous system (Fig. 21 B; Fig. 22 B). From stage 12 on, mRNA is also expressed in the developing gut (Fig. 21 C-E; Fig. 22 C,D). In late embryos, Otk and Otk2 mRNA are strongly expressed in the gut as well as the nervous system (Fig. 21 E, F; Fig. 22 E,F).

The expression of both mRNAs in segmentally repeated stripes is reminiscent of the expression of the segment polarity regulator Wingless. To check for a potential colocalization, embryos hybridized with FISH antisense probes were co-stained against Wingless. This clearly demonstrated that both Otk and Otk2 mRNA co-localize with Wingless protein (Fig. 21 G-I; Fig. 22 G-I). 


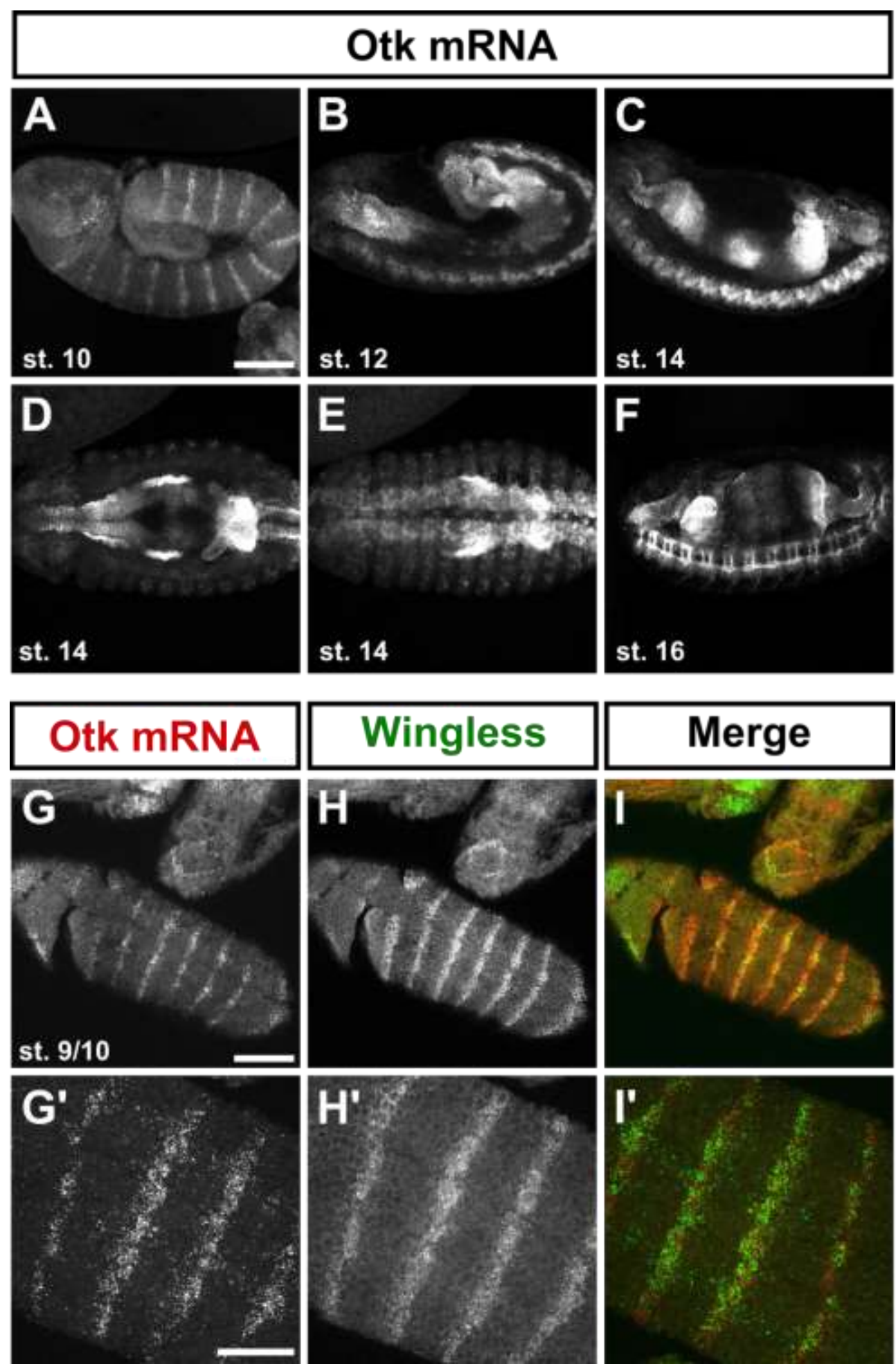

Fig. 21: mRNA expression of Off-track in embryos.

(A-F) Fluorescent in situ hybridization (FISH) with antisense probe against otk on white- embryos. (GI) Embryos hybridized with an antisense probe against otk were co-stained against Wingless. Stages of embryonic development are indicated. Anterior is to the left. Scale bars: A-I = $100 \mu \mathrm{m}, \mathrm{G}^{\prime}-\mathrm{I}^{\prime}=50 \mu \mathrm{m}$. 


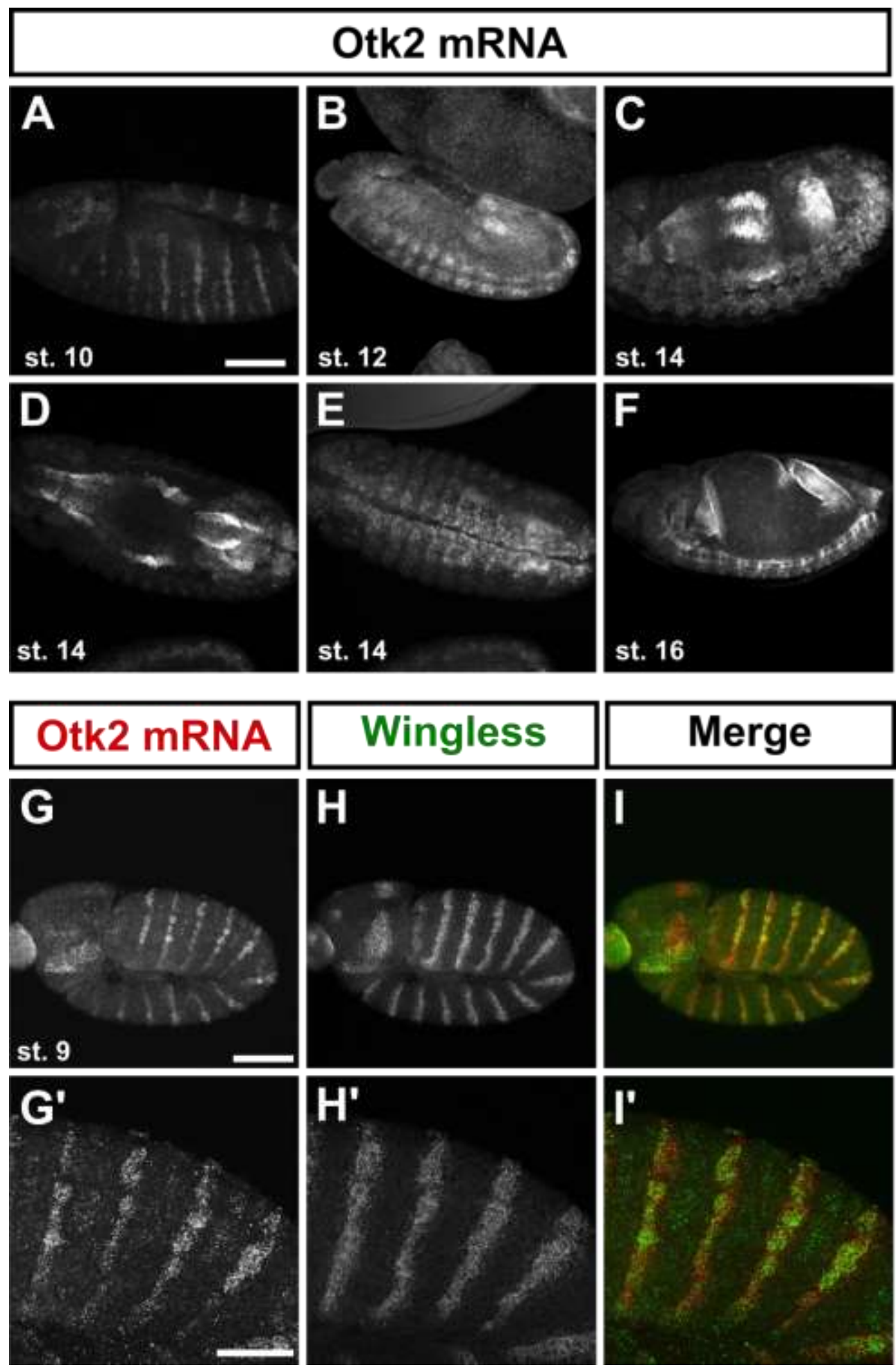

Fig. 22: mRNA expression of Off-track2 in embryos.

(A-F) Fluorescent in situ hybridization (FISH) with antisense probe against otk2 on white- embryos. (G-I) Embryos hybridized with an antisense probe against otk2 were co-stained against Wingless. Stages of embryonic development are indicated. Anterior is to the left. Scale bars: A-I = $100 \mu \mathrm{m}, \mathrm{G}^{\prime}-\mathrm{I}^{\prime}=$ $50 \mu \mathrm{m}$ 


\subsubsection{Protein expression of Off-track and Off-track 2 during embryonic development}

To determine the expression and subcellular localization of Otk and Otk2 during embryonic development, antibodies were generated against the two proteins. Whole mount immunofluorescent staining with an antibody raised against the extracellular domain of Otk confirmed the previously obtained expression data from reporter lines and FISH analysis. Otk is expressed in segmental stripes, in the developing nervous system and in the gut. Interestingly, it seems to be enriched in three domains along the developing gut (Fig. $23 \mathrm{G}, \mathrm{H}$ ). In late embryogenesis, high expression can be detected in the gut and the nervous system (Fig. 23 A-L). Corresponding results were obtained from stainings with a peptide antibody raised against Otk2 (Fig. 24 A-F). 


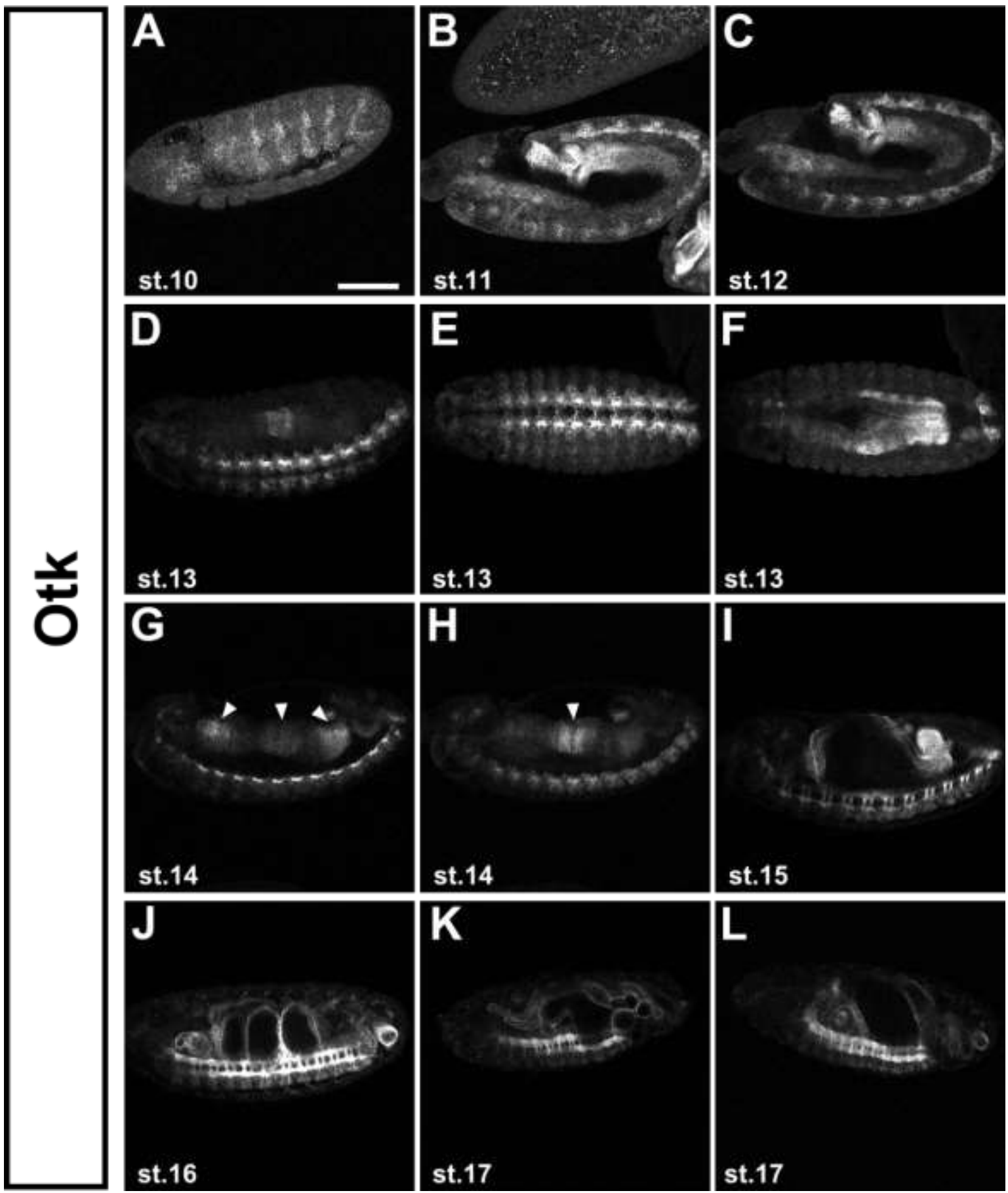

Fig. 23: Protein expression of Off-track in embryos.

(A-L) white- embryos were stained with an antibody against the extracellular domain of Otk. Stages of embryonic development are indicated. Anterior is to the left. Scale bar $=100 \mu \mathrm{m}$. 


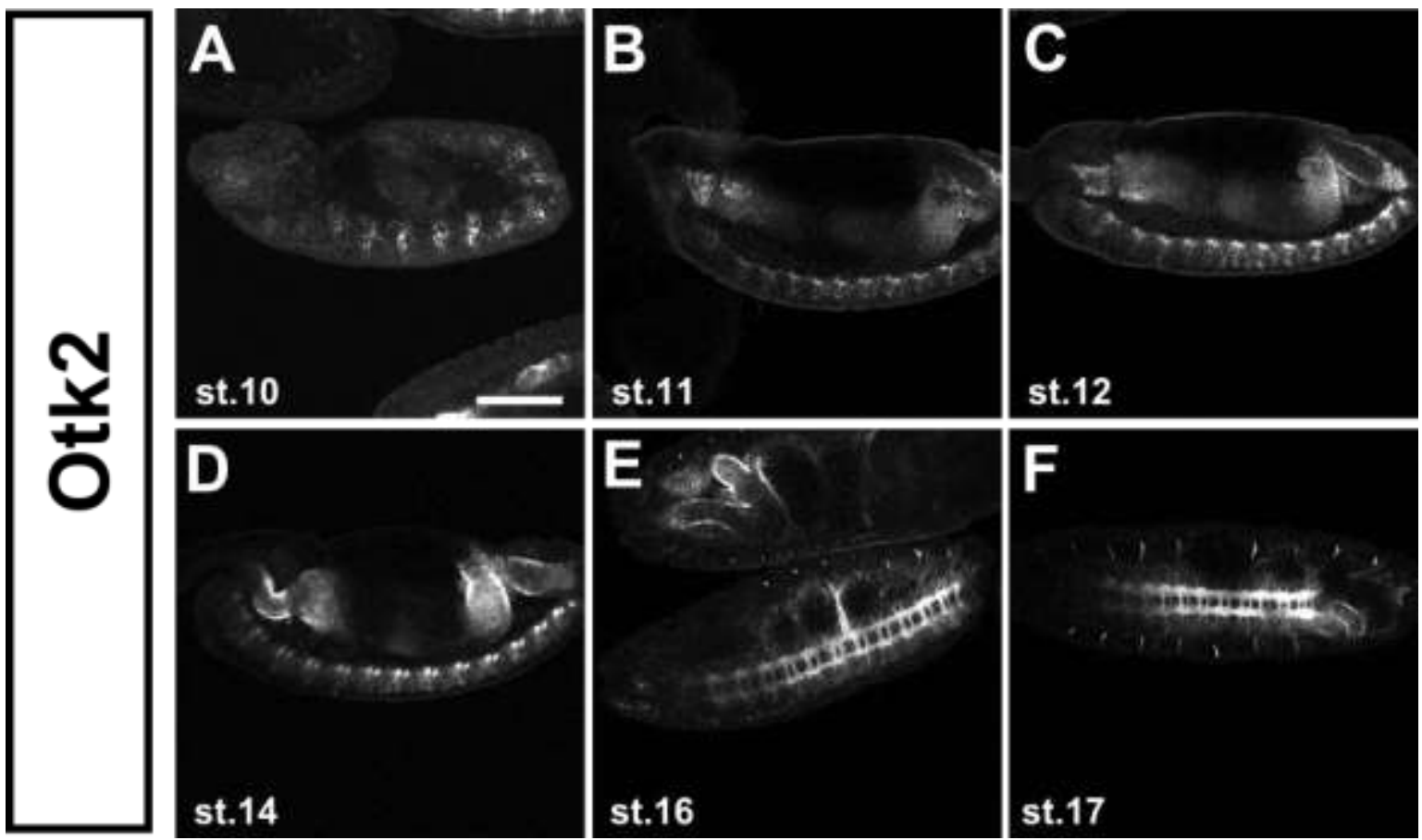

Fig. 24: Protein expression of Off-track2 in embryos.

(A-F) white- embryos were stained with a peptide antibody raised against Otk2. Stages of embryonic development are indicated. Anterior is to the left. Scale bar $=100 \mu \mathrm{m}$.

FISH analysis using antisense probes against Otk and Otk2 showed that the mRNA of both genes co-localizes with Wingless. To further validate this finding, co-stainings using antibodies against Otk and Wingless were performed (Fig. 25 A, B). These stainings showed that Otk is in fact expressed in cells located below the epithelial Wingless expressing cells (Fig. 25 B). Co-staining with the neuronal marker Elav revealed that Otk is expressed in segmentally repeated patches of neuroepithelial cells (Fig. 25 C). Remarkably, Otk seems to be expressed in the fraction of neuroblasts expressing Wingless and their neuronal progeny (Fig. 25 D). 

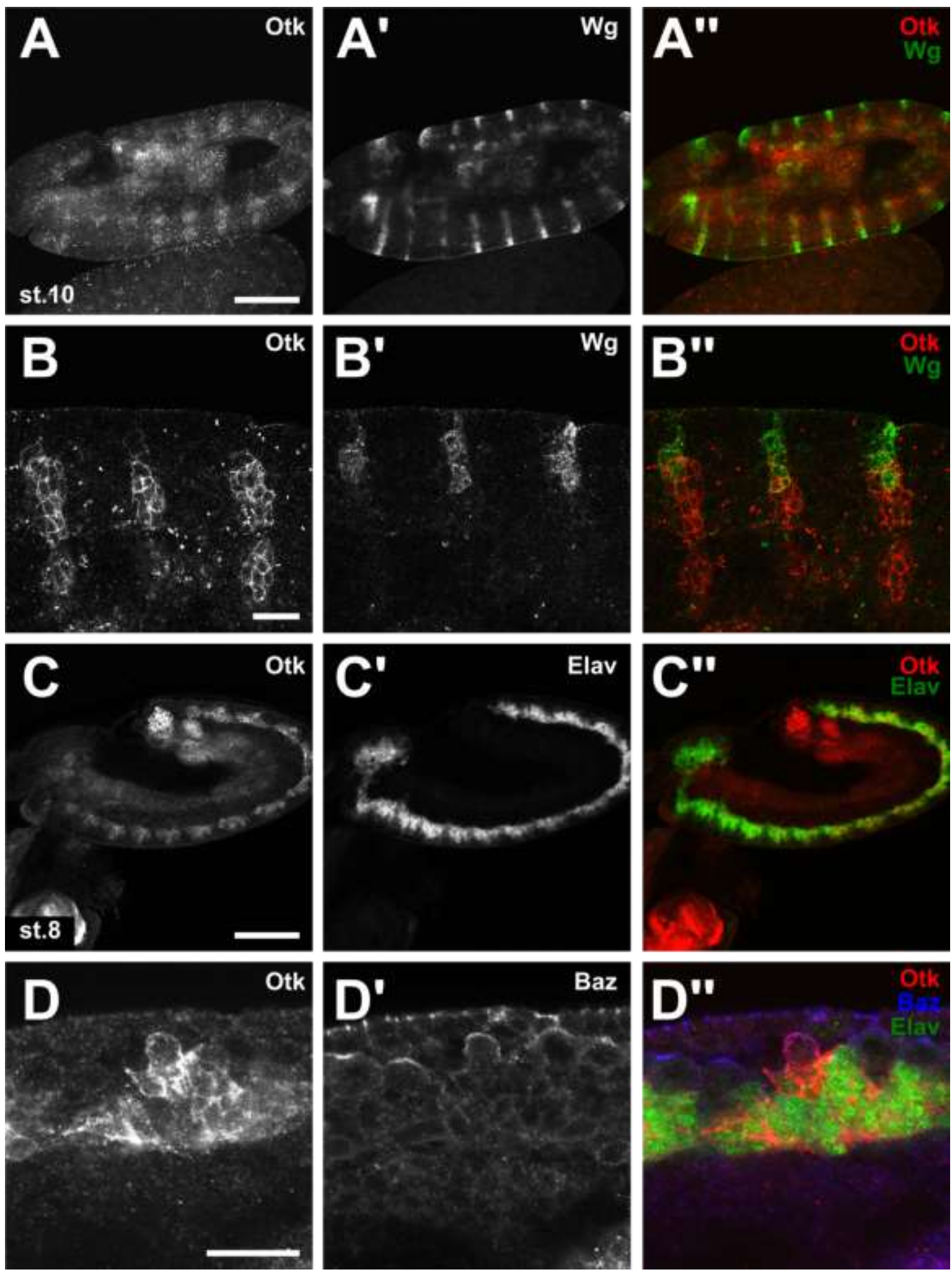

Fig. 25: Off-track is expressed in Wingless expressing neuroblasts and their neuronal progeny.

$(\mathbf{A}, \mathbf{B})$ white- embryos were stained against Otk and Wingless. (A) Otk is expressed in segmental stripes that colocalize with Wingless expression. (B) Higher magnification reveals expression of Otk in cells located below the Wingless expressing cells, presumably neuroblast progeny. $(\mathbf{C}, \mathbf{D})$ white- embryos were stained against Otk and the neuronal marker Elav. (C) Otk is expressed in neuroblast progeny. (D) Otk is expressed in a subset of neuroblasts and their progeny. Anterior is to the left. Scale bars: A, C $=100 \mu \mathrm{m}, \mathrm{B}, \mathrm{D}=20 \mu \mathrm{m}$. 
Wingless is not only expressed in the epithelium in segmentally repeated stripes, but also in parasegment 8 of the visceral mesoderm and the developing foregut and hindgut (Van den Heuvel et al, 1989). Interestingly, Otk is also highly expressed in the visceral mesoderm, partially co-localizing with Wingless (Fig. 26).
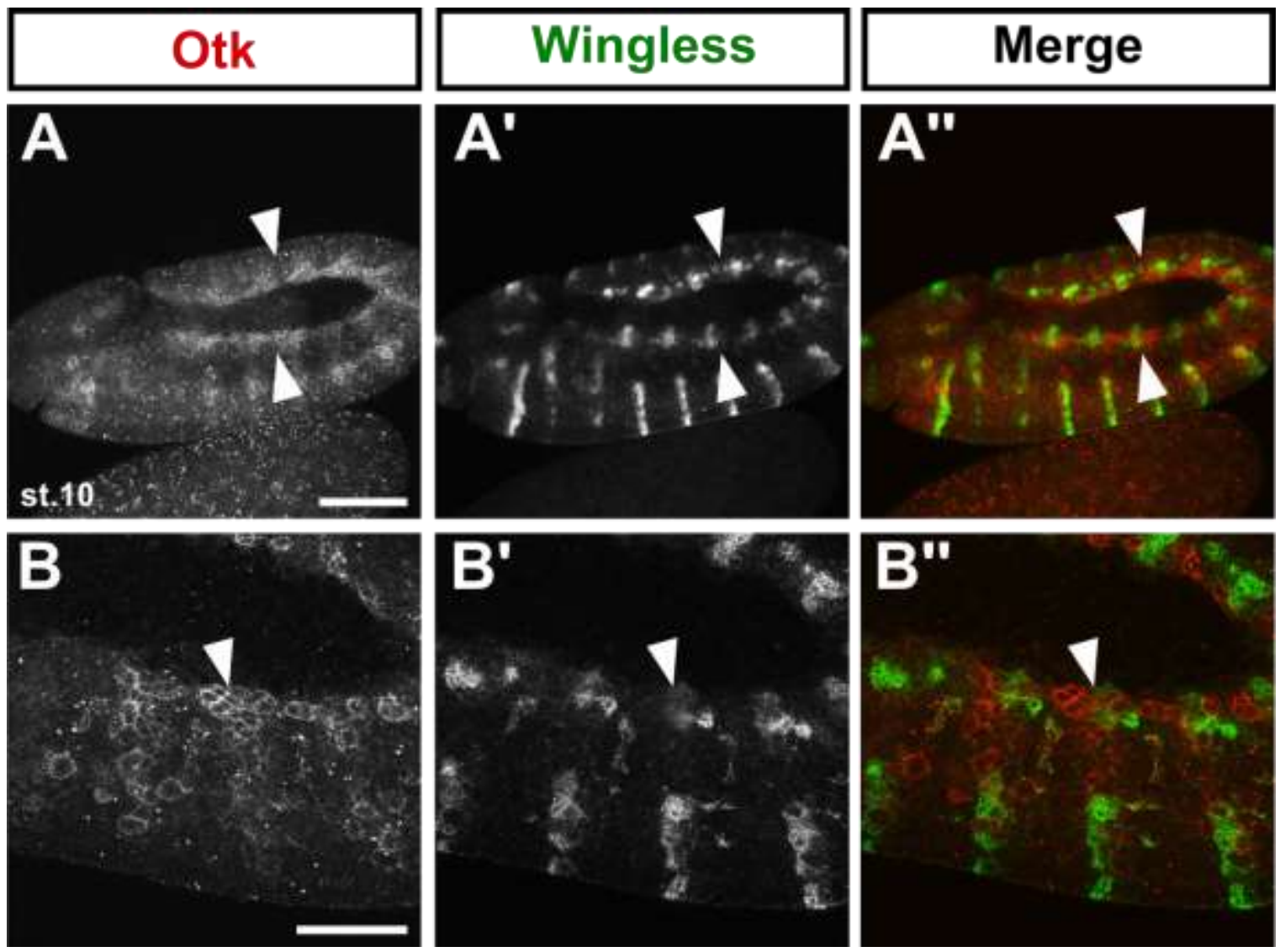

Fig. 26: Off-track is highly expressed in the visceral mesoderm.

white- embryos (stage 10) were stained against Otk and Wingless. (A) Wingless and Otk are expressed in the visceral mesoderm. (B) Otk is expressed along the entire visceral mesoderm and enriched in a segmental pattern. Anterior is to the left. Scale bars: $A=100 \mu \mathrm{m}, \mathrm{B}=50 \mu \mathrm{m}$.

As mentioned already, Otk is expressed in three domains along the developing gut. This expression has been shown to be controlled by the transcription factor Biniou (vertebrate FoxF) (Jakobsen et al, 2007). Wingless has been described to be expressed in three domains in the developing gut as well, namely the proventriculus, the Malphigian tubules and the region that will form the second midgut constriction (van den Heuvel et al, 1989; Immerglück et al, 1990), indicating that there might be a connection between Otk and Wingless signaling in embryonic gut development. Whole mount immunofluorescent staining showed that the three expression domains of Wingless and Otk indeed overlap, however, Otk expression appears to be broader 
in comparison to the restricted Wingless expression (Fig. 27). This might be explained by the fact that Wingless acts as a morphogen. Moreover, Otk expression appears to be stronger in the area adjacent to that of Wingless expression (Fig. 27 BD).

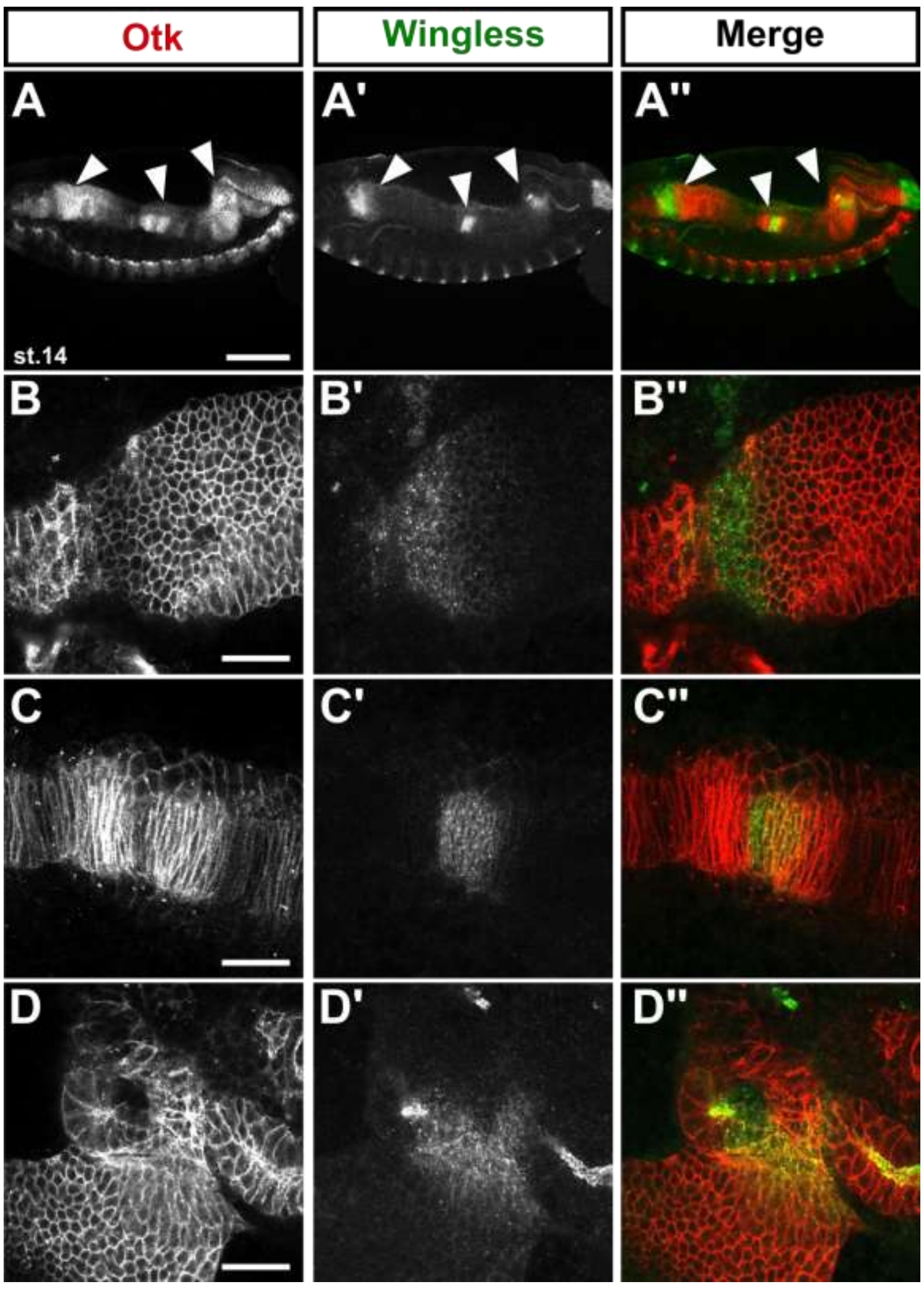

Fig. 27: Off-track is expressed in three regions in the embryonic gut. 
Fig. 27 (previous page): white- embryos (stage 14) were stained against Otk and Wingless. (A) Wingless is expressed in three stripes in the developing embryonic gut. Otk is expressed in three domains in the developing gut that colocalize with Wingless expression but are broader than the distinct Wingless stripes. (B-D) Higher magnification of the anterior (B), intermediate (C) and posterior (D) domain. Anterior is to the left. Scale bars: $A=100 \mu \mathrm{m}, \mathrm{B}=20 \mu \mathrm{m}$.

Initial immunofluorescent stainings revealed a high expression of Otk and Otk2 in the late embryonic nervous system. The expression overlaps with the marker for CNS axons, Bp102 (Fig. 28 A). However, in the course of these analyzes it became obvious that Otk and Otk2 are enriched at the anterior commissures compared to the posterior commissures (Fig. $28 \mathrm{~B}-\mathrm{G}$ ). Wnt5 is known to be expressed in the posterior commissure and acts as a repellent ligand for Derailed-expressing neurons that cross the midline along the anterior commissure (Yoshikawa et al, 2003; Fradkin et al, 2004). In this study, I detected high levels of Wnt5 protein in the anterior commissures, colocalizing with Otk (Fig. $28 \mathrm{H}, \mathrm{I}$ ) and most likely reflecting the bound pool of Wnt5 protein (Jasprien Nordermeer, personal communication). 


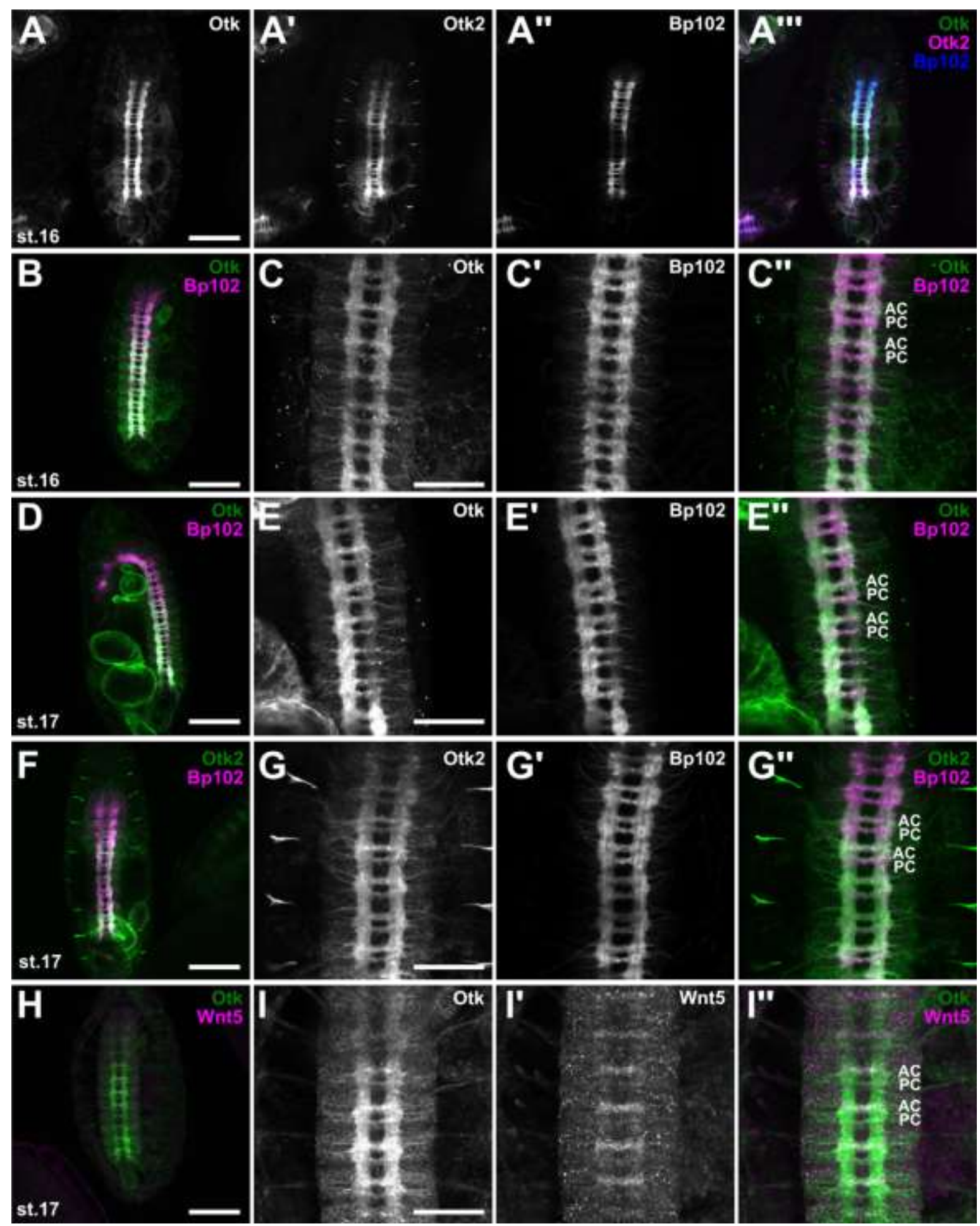

Fig. 28: Otk and Otk2 are expressed in the late embryonic nervous system and enriched at anterior commissures.

(A) white- embryos were stained against Otk, Otk2 and Bp102, an antibody against CNS axons. Otk and Otk2 are highly expressed in the nervous system and the expression colocalizes with Bp102 expression. (B-D) white- embryos were stained against Otk and Bp102. Higher magnifcation reveals an enrichment of Otk at anterior commissures at stage 16 (C), which becomes even more prominent at stage 17 (E). (F,G) white- embryos were stained against Otk2 and Bp102. Higher magnifcation reveals an enrichment of Otk2 at anterior commissures at stage $17 \mathbf{( G ) . ~ ( H , I ) ~ w h i t e - ~ e m b r y o s ~ w e r e ~ s t a i n e d ~}$ against Otk and Wnt5. Wnt5 is enriched at anterior commissures (I'), clearly colocalizing with Otk (I'). Stages of embryonic development are indicated. AC, anterior commissure; PC, posterior commissure. Anterior is to the top. Scale bars: A,B,D,F,H $=100 \mu \mathrm{m} ; \mathrm{C}, \mathrm{E}, \mathrm{G}, \mathrm{I}=50 \mu \mathrm{m}$. 


\subsection{Expression of Off-track in Drosophila Wnt family mutants}

To determine whether Otk expression itself might be a target of Wnt signaling, I compared Otk expression in different wht mutant backgrounds (Fig. 29). Interestingly, Otk does not seem to be regulated by Wingless or Wnt4, since both the segmental localization in early embryos as well as the localization in the developing gut and nervous system were not affected (Fig. 29 A-D). Interestingly, the expression of Otk in the nervous system appeared to be slightly reduced in wht5 mutant embryos (Fig. 29 E) and overall Otk expression levels were strongly reduced in embryos homozygous mutant for wht2 (Fig. 29 F-H). This overall reduction did not become stronger in wnt2,wg and wnt2,wnt4 double mutants, indicating that wg and wnt4 indeed do not have any influence on Otk expression (Fig. 29 I,J). 

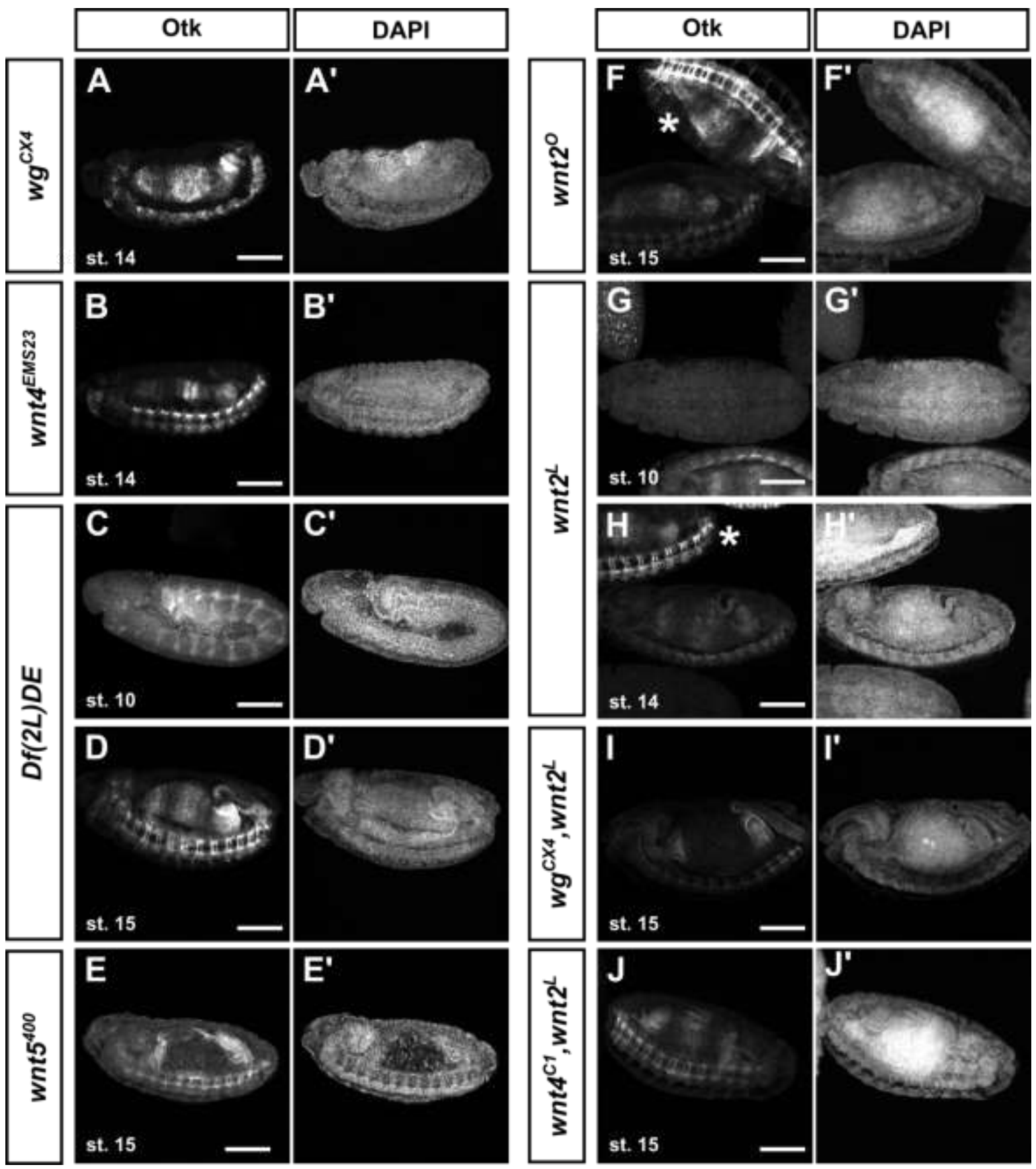

Fig. 29: Localization of Otk in embryos homozygous mutant for different Wnt family members.

Homozygous mutant embryos of the indicated genotypes were stained against Otk. In embryos homozygous mutant for $w g$ (A) and Wnt4 (B), as well as a deficiency removing both $w g$ and wht4 (C,D) the expression pattern of Otk is not changed. (E) In homozygous mutant wht 5 embryos the expression in the nervous system is slightly reduced. (F-H) Otk expression is decreased in embryos homozygous mutant for wnt2. A homozygous compared to a heterozygous (asterisk) mutant embryo are depicted for comparison $(\mathbf{F}, \mathbf{H})$. $(\mathbf{I}, \mathbf{J})$ Double mutants of the indicated genotypes display the same phenotype as the wnt2 single mutant (F-H). Anterior is to the left. Scale bars $=100 \mu \mathrm{m}$. 


\subsection{Generation of otk and otk2 null alleles}

To further investigate the function of Otk and Otk2, null alleles were generated for both genes as well as a double knock-out. For this purpose the full coding sequence of otk as well as otk2 were removed via FLP/FRT-mediated excision (Parks et al, 2004). The peculiar chromosomal localization of both genes in tandem offers an easy way to generate a double knock-out for both genes using the same technique as for the otk and otk2 single knock-out. This method utilizes the ability of FLP recombinase to induce recombination between two FRT sites positioned in trans on two complementary chromosomes. Three suitable transposon insertion lines containing FRT sites were available from the Harvard stock collection. The P(XP)d01360 element is located upstream of the 5'UTR of otk and the PBac(RB)e03992 element is inserted downstream of the 3'UTR of otk. The PBac(PB)c01790 element is located in the second exon of Mppe, a gene located upstream of otk2 and P(XP)d01360 is downstream of the $3^{\prime} \mathrm{UTR}$ of otk2 (Fig. $30 \mathrm{~A}$ ). FLP recombinase-caused deletion of the genomic region between the FRT sites in P(XP)d01360 and PBac(RB)e03992 was used to remove the coding region of otk. Likewise, the genomic region of otk2 was removed by recombination between the FRT sites located in P(XP)d01360 and $\mathrm{PBac}(\mathrm{PB}) \mathrm{c01790}$. Finally, excision of the genomic region between the FRT sites in $\mathrm{PBac}(\mathrm{PB}) \mathrm{c} 01790$ and $\mathrm{PBac}(\mathrm{RB}) \mathrm{e} 03992$ deleted the genomic region of both otk and otk2.

Parts of the Mppe gene upstream of otk2 were also removed during recombination using $\mathrm{PBac}(\mathrm{PB}) \mathrm{c01790}$. Mppe is not an essential gene and encodes for a metallophosphoesterase that functions in Rhodopsin Rh1 deglycosylation (Cao et al, 2011).

Mutant flies potentially carrying one of the desired deletions were selected by the loss of the mini-white genes and/or PCR as described in 2.5.5. The following alleles could be recovered: $o t k^{A 1}$ removes the genomic region of $o t k$ and $o t k 2{ }^{c 26}$ the genomic region of $o t k 2$, while otk,otk2 ${ }^{D 72}$ is the double mutant of both genes (Fig. $30 \mathrm{~A}$ ). As the three putative deletion lines were all homozygous viable, loss of the respective genes was tested by several methods. Each deletion was verified by PCR on adult genomic DNA (Fig. 30 B-D). First, PCRs were done on the transition from the genomic region to the ends of the P-elements to verify that the inner ends of the P-elements are deleted, while the outer ends still are left after recombination has taken place. Furthermore, 
PCR was performed with primer pairs that encompass the entire deleted regions (Fig. 30 B-D). Subsequently, the resulting PCR bands were purified and sequenced.

To further prove that the obtained mutant lines indeed represent null alleles of the respective genes, I performed Western Blots with protein extracts from homozygous mutant embryos and the corresponding original transposon lines as well as chromosomal deficiencies as controls (Fig. 30 E). Detection with the antibody against the extracellular domain of Otk resulted in a band of approximately $120 \mathrm{kDa}$, corresponding to full-length Otk. As expected, this could be detected in all lanes except for $o t k^{A 1}$, otk,otk2 ${ }^{D 72}$ and the deficiency lines. Interestingly, no protein could be detected in extracts from the original transposon line PBac(RB)e03992 either. Additional analysis will have to confirm if this line might represent an allele of otk. Detection with the peptide antibody against Otk2 resulted in a band of approximately $50 \mathrm{kDa}$, corresponding to full-length Otk2. Likewise, Otk2 protein could be detected in all lanes except for otk2 $2^{C 26}$, otk,otk2 $2^{D 72}$ and the deficiency lines (Fig. 30 E). The slight band in lane 7 is due to imprecise loading of the gel.

In addition, whole mount immunofluorescent stainings were performed on homozygous mutant embryos (Fig. $31 \mathrm{~A}-\mathrm{C}$ ). These analyses clearly demonstrated that no Otk protein can be detected in otk ${ }^{A 1}$ embryos, while Otk2 localization is normal (Fig. $31 \mathrm{~A}$ ). In agreement with this, no Otk2 protein could be detected in otk2 ${ }^{\text {C26 }}$ embryos, while Otk localization is not changed (Fig. 31 B). Finally, neither Otk nor Otk2 could be detected in otk,otk2D72 embryos (Fig. $31 \mathrm{C}$ ). I concluded that the obtained alleles otk ${ }^{A 1}$, otk2 $2^{C 26}$ and otk,otk2D72 are indeed null alleles for the respective genes. 
A

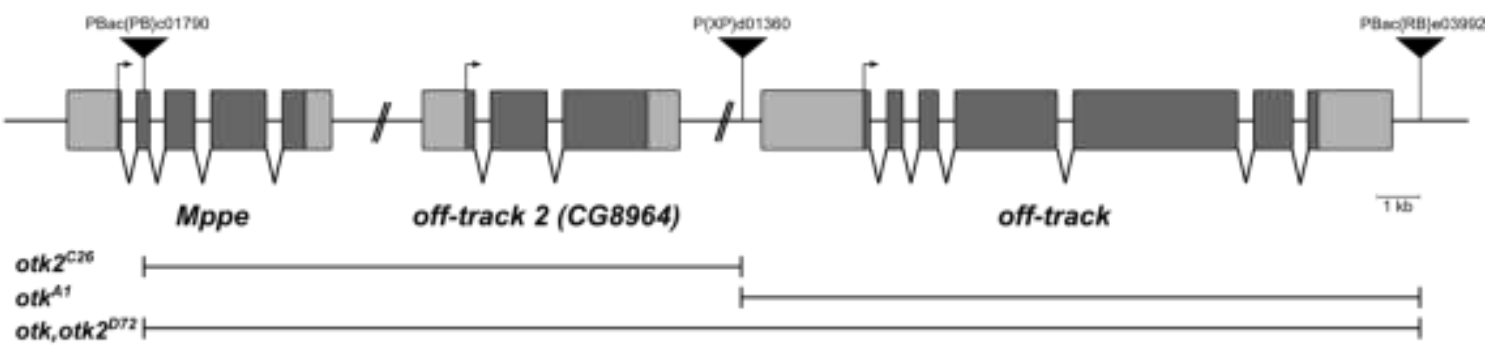

B

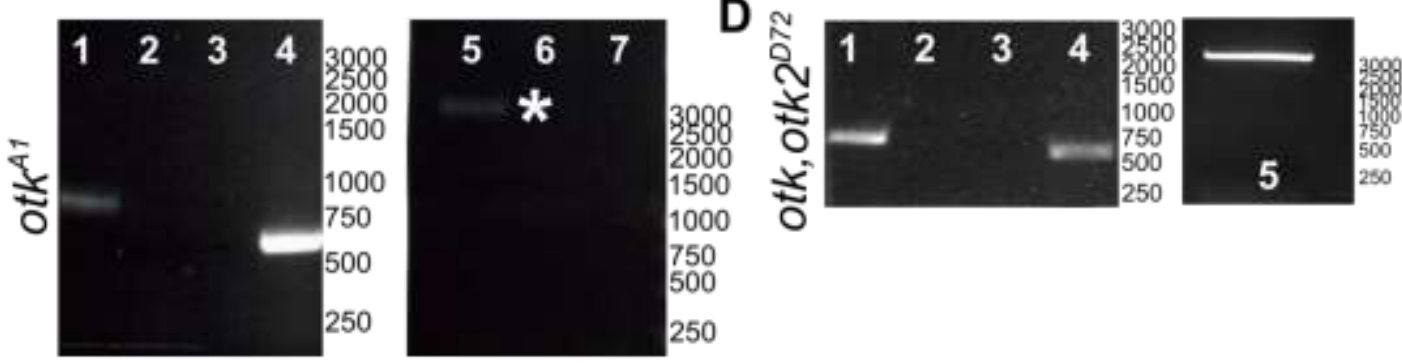

C

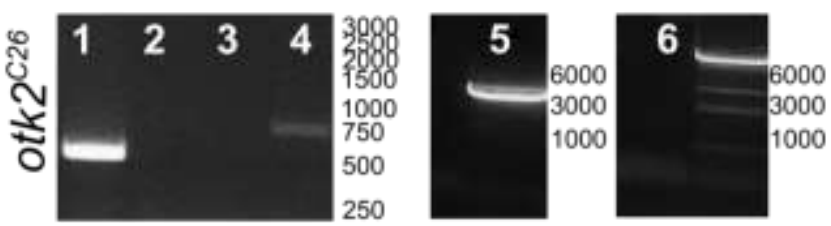

E

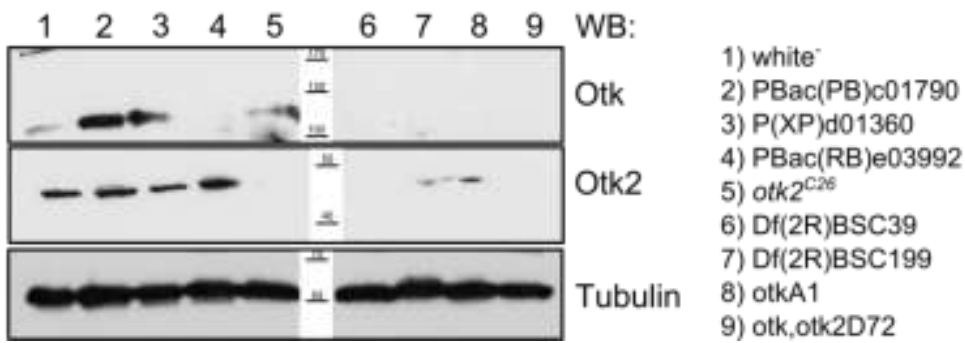

Fig. 30: Generation of otk and otk2 null alleles.

(A) Overview of the genomic region of otk, otk2 and the neighboring gene Mppe located on chromosome 2R. Insertion positions of the three P-element transposons utilized are shown. Null alleles for otk and otk2 alone as well as both otk and otk2 were generated via FLP/FRT mediated recombination between FRT sites contained in P(XP)d01360 and PBac(RB)e03992 (otk ${ }^{A 1}$ ), PBac(PB)c01790 and P(XP)d01360 (otk2 ${ }^{\text {c26) }}$ ) or PBac(PB)c01790 and PBac(RB)e03992 (otk,otk2 272). (B-D) Verification of the three generated alleles by PCR. (B) Verification of the otk ${ }^{A 1}$ deletion by PCR on genomic DNA from adult flies. Lane 1-4: The transition between the ends of the P-elements and the genomic region was amplified. PCR was done on the left (1) and right (2) end of P(XP)d01360 and on the left (3) and right (4) end of PBac(RB)e03992. Missing bands indicate the loss of the inner Pelement ends. Lane 5 (asterisk): The deleted site was amplified with primers binding upstream and downstream of the whole deletion in the genomic region. The band obtained was purified and sequenced. Adult fly genomic DNA from P(XP)d01360 (lane 6) and PBac(RB)e03992 (lane 7) was used as control. Missing bands can be explained by the large size of the expected fragments. (C) Verification of the otk2 ${ }^{226}$ deletion by PCR on genomic DNA from adult flies. Lane 1-4: The transition between the ends of the P-elements and the genomic region was amplified. PCR was done on the left (1) and right (2) end of PBac(PB)c01790 and on the left (3) and right (4) end of P(XP)d01360. Missing bands indicate the loss of the inner P-element ends. Furthermore, the deleted site was amplified with primers binding upstream of the deletion in the genomic region and the residual transposon (lane 5) as well as primers binding in the residual transposon and downstream of the deletion in the genomic region 
(lane 6). The bands obtained were purified and sequenced. (D) Verification of the otk,otk2D72 deletion by PCR on genomic DNA from adult flies. Lane 1-4: The transition between the ends of the P-elements and the genomic region was amplified. PCR was done on the left (1) and right (2) end of PBac(PB)c01790 and on the left (3) and right (4) end of PBac(RB)e03992. Missing bands indicate the loss of the inner P-element ends. Lane 5: The deleted site was amplified with primers binding upstream and downstream of the whole deletion in the genomic region. The band obtained was purified and sequenced. (E) Protein extracts from embryos of the indicated genotype were analyzed by Western Blot with the antibodies shown. Protein extracts from embryos of the original transposon stocks (lane 2-4) as well as from homozygous embryos from chromosomal deficiency lines (lane 6 and 7) were used as controls. No Otk protein was detected in otk ${ }^{A 1}$ (lane 8) and otk,otk2 ${ }^{D 72}$ (lane 9) homozygous mutant embryos as well as in embryos carrying the PBac(RB)e03992 P-element (lane 4). No Otk2 protein was detected in otk2 ${ }^{C 26}$ (lane5) and otk,otk2 ${ }^{D 72}$ (lane 9) homozygous mutant embryos. An antibody against tubulin was used as a loading control.

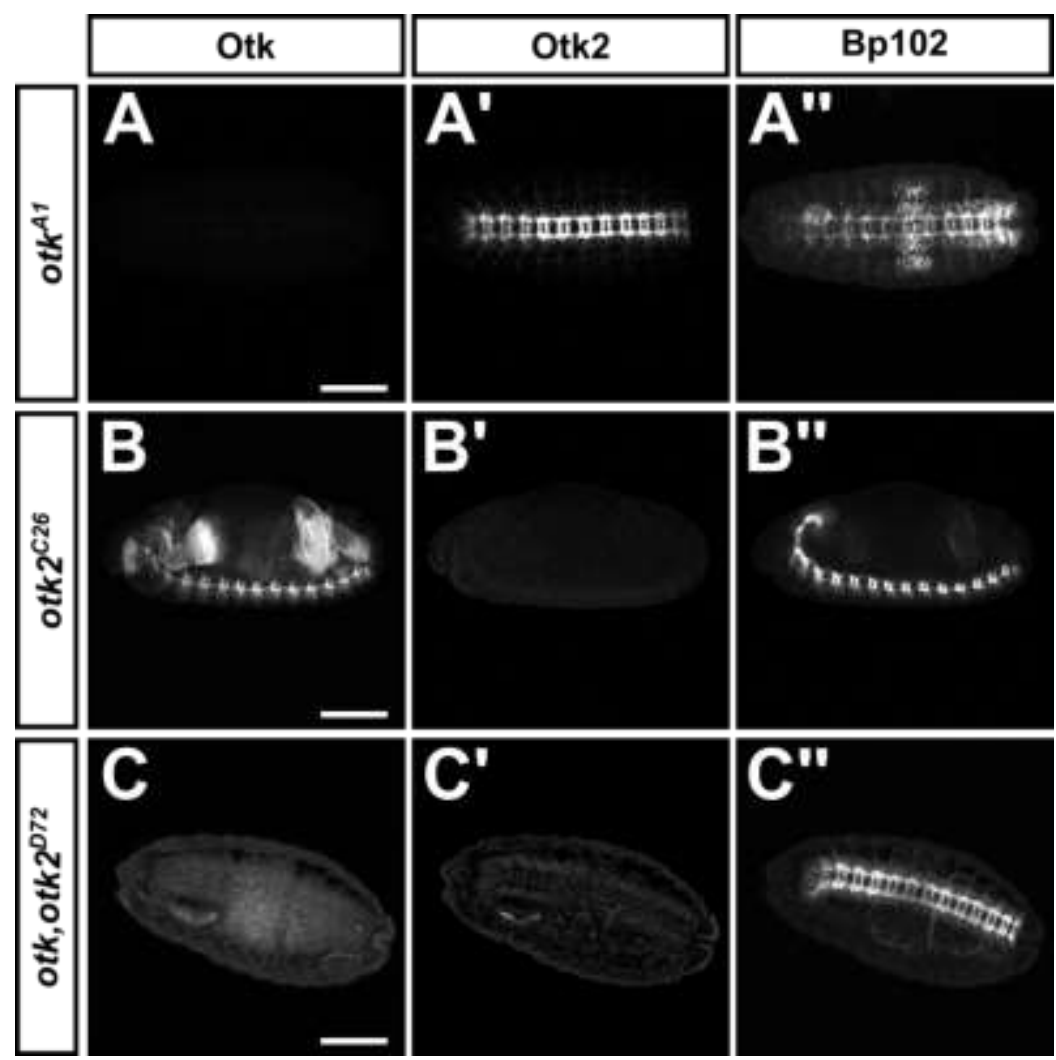

Fig. 31: Verification of otk and otk2 null alleles.

(A-C) Verification of the three generated alleles by whole mount immunofluorescent stainings. Homozygous mutant embryos of the indicated genotypes were stained with antibodies against Otk, Otk2 and the CNS axon marker Bp102 as control. (A) In otk ${ }^{A 1}$ homozygous mutant embryos no Otk protein can be detected, but the staining for Otk2 is normal. (B) In otk2 ${ }^{26}$ homozygous mutant embryos no Otk2 protein can be detected, but the staining for Otk is normal. (C) In otk,otk2 ${ }^{D 72}$ homozygous mutant embryos neither Otk nor 0tk2 protein can be detected. Anterior is to the left. Scale bar $=100 \mu \mathrm{m}$. 


\subsection{Characterization of otk and otk2 null alleles}

Flies homozygous mutant for otk ${ }^{A 1}$ or otk ${ }^{C 26}$ were homozygous viable and did not show obvious phenotypic defects. This strongly disagrees with reports on the previously generated ot $^{3}$ allele (Winberg et al, 2001; Cafferty et al, 2004; Peradziryi et al, 2011). Interestingly, also flies homozygous mutant for otk,otk2 ${ }^{D 72}$ were viable, but male sterile and no homozygous stock could be established. To test whether loss of any of the genes leads to subtle defects several approaches were performed.

\subsubsection{Novel otk and otk $k^{2}$ loss of function mutants are homozygous viable}

To analyze if loss of either otk, otk2 or both genes leads to defects that cause a decrease in viability, I determined the hatching rate of embryos homozygous mutant for otk $^{A 1}$, otk ${ }^{C 26}$ and otk,otk2D72. In case of the double deletion, maternally mutant embryos derived from homozygous mutant otk,otk2 ${ }^{D 72}$ mothers and heterozygous fathers were used. The average viability of embryos homozygous for each of the deletions was comparable to that of white- embryos as well as the original transposon lines (Fig. 32).

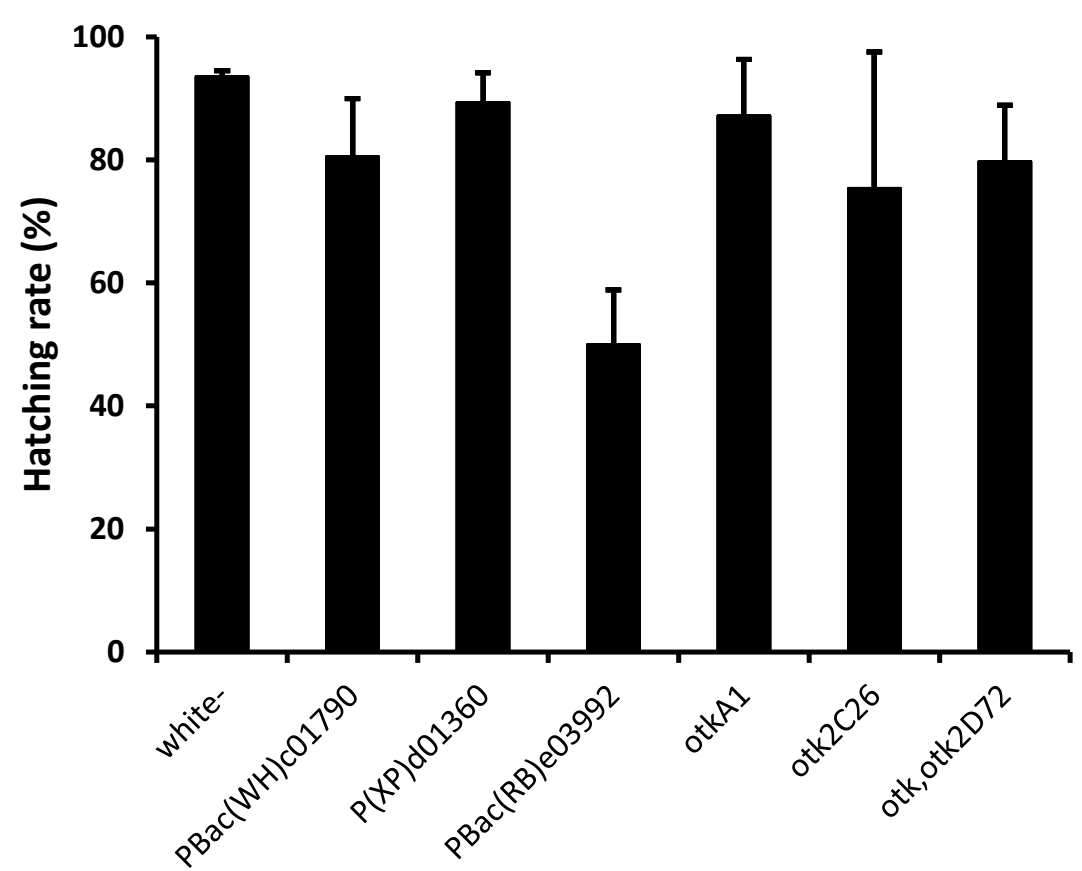

Fig. 32: Novel otk and otk2 loss of function mutants are homozygous viable and do not display any PCP or wing margin defects.

(A) Lethality assays were performed with embryos of the indicated genotypes. Embryos were collected and allowed to develop for two days at $25{ }^{\circ} \mathrm{C}$. The number of hatched embryos was determined. The experiment was repeated three times. Error bars represent the standard error of the mean. 


\subsubsection{Life span is not affected in novel otk and otk2 loss of function mutants}

To investigate if loss of either otk, otk2 or both genes alone is sufficient to extend or decrease life expectancy, flies homozygous mutant for otk ${ }^{A 1}$ otk ${ }^{C 26}$ and otk,otk2 $2^{D 72}$ were examined. No difference in life span could be seen for any of the genotypes observed (Fig. 33), suggesting that there is no relationship between Otk or Otk2 function and life expectancy.
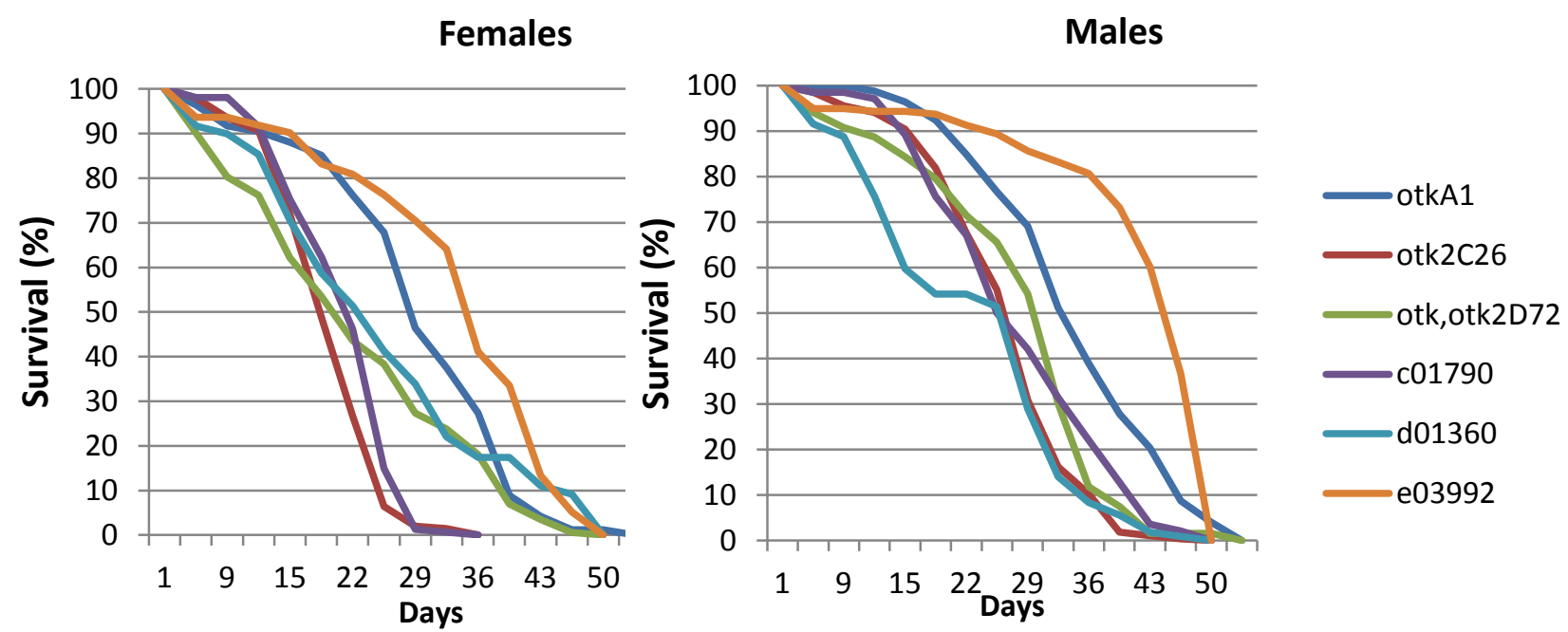

Fig. 33: Life span is not affected in novel otk and otk2 loss of function mutants.

Survival rates of homozygous flies of the indicated genotypes were recorded. No difference can be observed between the deletion lines compared to the original transposon stocks.

\subsubsection{Homozygous otk ${ }^{A 1}$ and otk,otk2 ${ }^{D 72}$ flies do not display any PCP or wing margin defects}

Defects in planar cell polarity (PCP) signaling usually can be recognized by disorganization of wing hairs as well as thorax and leg bristles of adult flies (Axelrod \& McNeill, 2002; Zallen, 2007). In contrast, failure in canonical Wingless signaling leads to wing margin defects (Couso et al, 1994; Zhang \& Carthew, 1998). Furthermore, the photoreceptors in each ommatidium of the adult eye are arranged in an arrow-like manner with the arrow tips pointing towards the equator of the eye (Fig. 35 B). This organization is also frequently disturbed in PCP mutants (Axelrod \& McNeill, 2002; Zallen, 2007).

To study if any of these defects can be found in otk or otk2 mutants, wings of adult flies were examined (Fig. $34 \mathrm{~A}-\mathrm{E}$ ). In contrast to the function of PTK7 in vertebrate PCP signaling (Lu et al, 2004; Shnitsar \& Borchers, 2008; Yen et al, 2009; Peradziryi et al, 2011), loss of neither otk or otk2 alone (Fig. 34 D, data for otk2 not shown) nor of both otk and otk2 (Fig. $34 \mathrm{E}$ ) leads to any defects in wing hair orientation compared 
to original P-element lines as controls (Fig. $34 \mathrm{~B}, \mathrm{C}$ ). In agreement with this finding, ommatidia organization was not disturbed either in otk ${ }^{A 1}$, otk ${ }^{C 26}$ and otk,otk2 ${ }^{D 72}$ flies compared to wild type (Fig. $35 \mathrm{~A}-\mathrm{D}$, data for otk2 not shown).
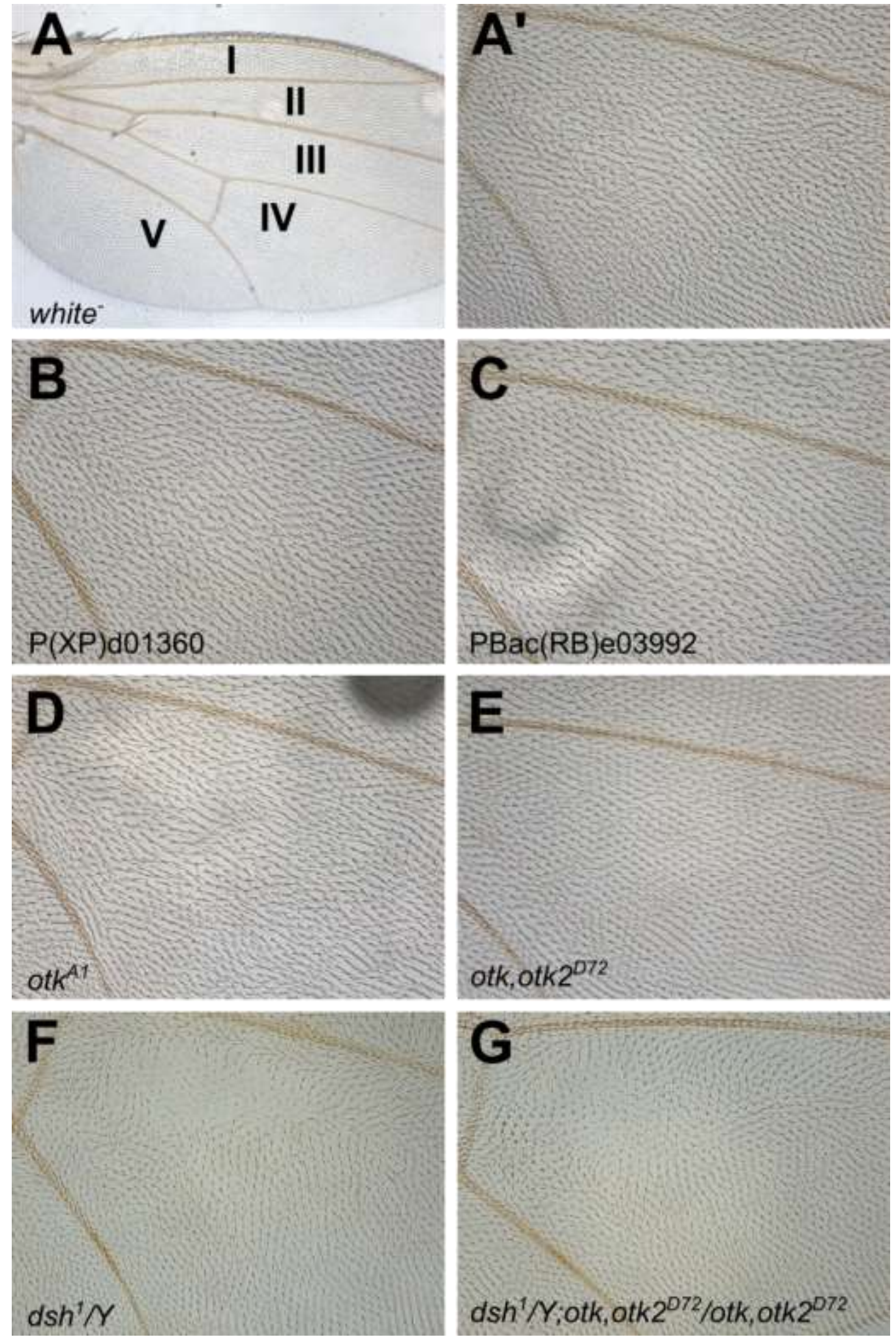

Fig. 34: Homozygous otk ${ }^{A 1}$ and otk,otk2 ${ }^{D 72}$ flies do not display any wing PCP or margin defects.

Wings from adult flies homozygous mutant for $\operatorname{otk}^{A 1}$ (D) and $o t k$,otk2 ${ }^{D 72}$ (E) (no maternal contribution) do not show any defects compared to white- flies or the original transposon lines (A-C). (F) Wings from $d s h 1 / Y$ flies as an example for a characteristic PCP phenotype. The phenotype is unchanged in 
double mutants for $d s h 1$ and otk,otk2D72 (G). (A) shows 10X magnifications of adult wings and the five regions of the wing are indicated. (A',B-G) display 25X magnifications of region IV of the respective wing. Anterior is up and proximal is to the left.

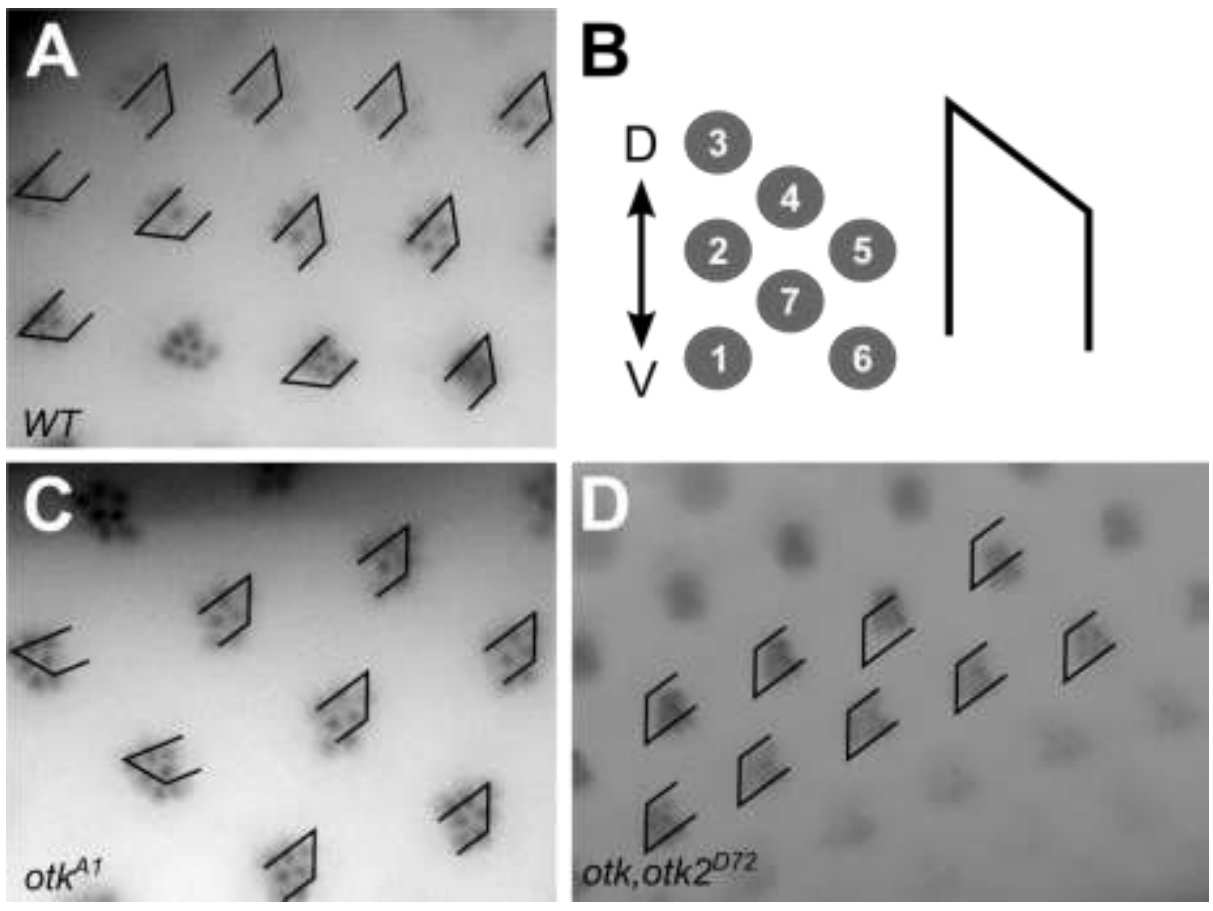

Fig. 35: Eyes of homozygous otk ${ }^{A 1}$ and otk,otk2 ${ }^{D 72}$ flies do not display any PCP defects.

Eyes from adult flies homozygous mutant for otk $^{A 1}$ (C) and otk,otk2 ${ }^{D 72}$ (D) do not show any defects compared to wild type (A). (B) Schematic representation of the ommatidia structure of an adult eye.

\subsection{4 otk,otk $2^{D 72}$ homozygous mutants flies are male sterile}

As already mentioned, adult flies homozygous mutant for otk,otk2D72 were male sterile and this phenotype was fully penetrant. Apparently, both copies of both genes need to be removed, since transheterozygous otk,otk2D72/otk ${ }^{A 1}$ or otk,otk2 ${ }^{D 72} /$ otk2 $^{C 26}$ males were fertile (Table 16). The sterility could be rescued by introduction of a fulllength Otk-GFP transgene under UASp promoter, expressed by the ubiquitous driver daughterless::Gal4 (Table 17). In contrast, an Otk2-GFP transgene under UASp promoter did not rescue sterility. This suggests that even though the localization of the construct was normal (data not shown), the function of Otk2 is disturbed by the introduction of the C-terminal GFP tag. 
Table 16: otk,otk2D72 homozygous mutant males are male sterile.

\begin{tabular}{|c|c|}
\hline otk,otk2 $2^{D 72} /$ otk,otk2 ${ }^{D 72}$ & Male sterile \\
\hline otk $^{A 1} /$ otk $^{A 1}$ & Fertile \\
\hline otk $2^{\mathrm{C26}} /$ otk $2^{\mathrm{C26}}$ & Fertile \\
\hline otk,otk2 ${ }^{D 72} /$ otk $^{A 1}$ & Fertile \\
\hline otk,otk2 $2^{D 72} /$ otk2 $2^{c 26}$ & Fertile \\
\hline otk, otk2 $^{D 72} / D f(2 R) B S C 39$ & Male Sterile \\
\hline otk,otk2 ${ }^{D 72} / D f(2 R) B S C 199$ & Male sterile \\
\hline
\end{tabular}

Table 17: Sterility otk,otk2 ${ }^{D 72}$ homozygous mutants males can be rescued by 0 tk transgenes.

\begin{tabular}{|c|c|}
\hline otk,otk2 $2^{D 72} /$ otk, otk2 $2^{D 72}$ & Male sterile \\
\hline otk,otk2 $2^{D 72} /$ otk,otk2 ${ }^{D 72}$; da / UASp::Otk-GFP29 & Fertile \\
\hline otk,otk2 ${ }^{\text {D72 }} /$ otk,otk2 ${ }^{\text {D72 }}$; da / UASp::OtkDeltaC-GFP14 & Male sterile \\
\hline otk,otk2 ${ }^{D 72} /$ otk,otk2 ${ }^{\text {D72 }}$; da / UASp::OtkDeltaEx-GFP2O & Male sterile \\
\hline otk,otk2 ${ }^{D 72} /$ otk,otk2 ${ }^{D 72} ;$ da / UASp::Otk2-GFP37 & Male sterile \\
\hline otk,otk2 ${ }^{D 72} /$ otk,otk2 $2^{D 72}$; da / UASt::Otk-GFP & not done \\
\hline otk,otk2 ${ }^{D 72} /$ otk, otk2 ${ }^{D 72} ;$ da / UASt::Otk2-RFP & not done \\
\hline
\end{tabular}

To analyze whether sterility is caused by defects in sperm development, testes from males heterozygous and homozygous mutant for otk,otk2 $2^{D 72}$ were dissected and stained with Vasa, a marker for germline stem cells (Lasko \& Ashburner, 1990) and FasciclinIII, which marks the hub (Gönczy \& DiNardo, 1996) (Fig. 36 A-C). The hub consists of non-dividing stromal cells constituting the stromal niche for the germline stem cells and cyst stem cells (De Cuevas \& Matunis, 2011). Both markers localize normally in testes homozygous mutant for otk,otk2 ${ }^{D 72}$ (Fig. $36 \mathrm{~B}, \mathrm{C}$ ), indicating that sterility is not caused by any defects in stem cell regulation. Furthermore, co-staining with the DNA marker DAPI revealed that all stages of sperm development, which can be distinguished by their characteristic packaging of the DNA, are present in testes from males homozygous mutant for otk,otk2 ${ }^{D 72}$ (Fig. 36 B'", C'”'). 

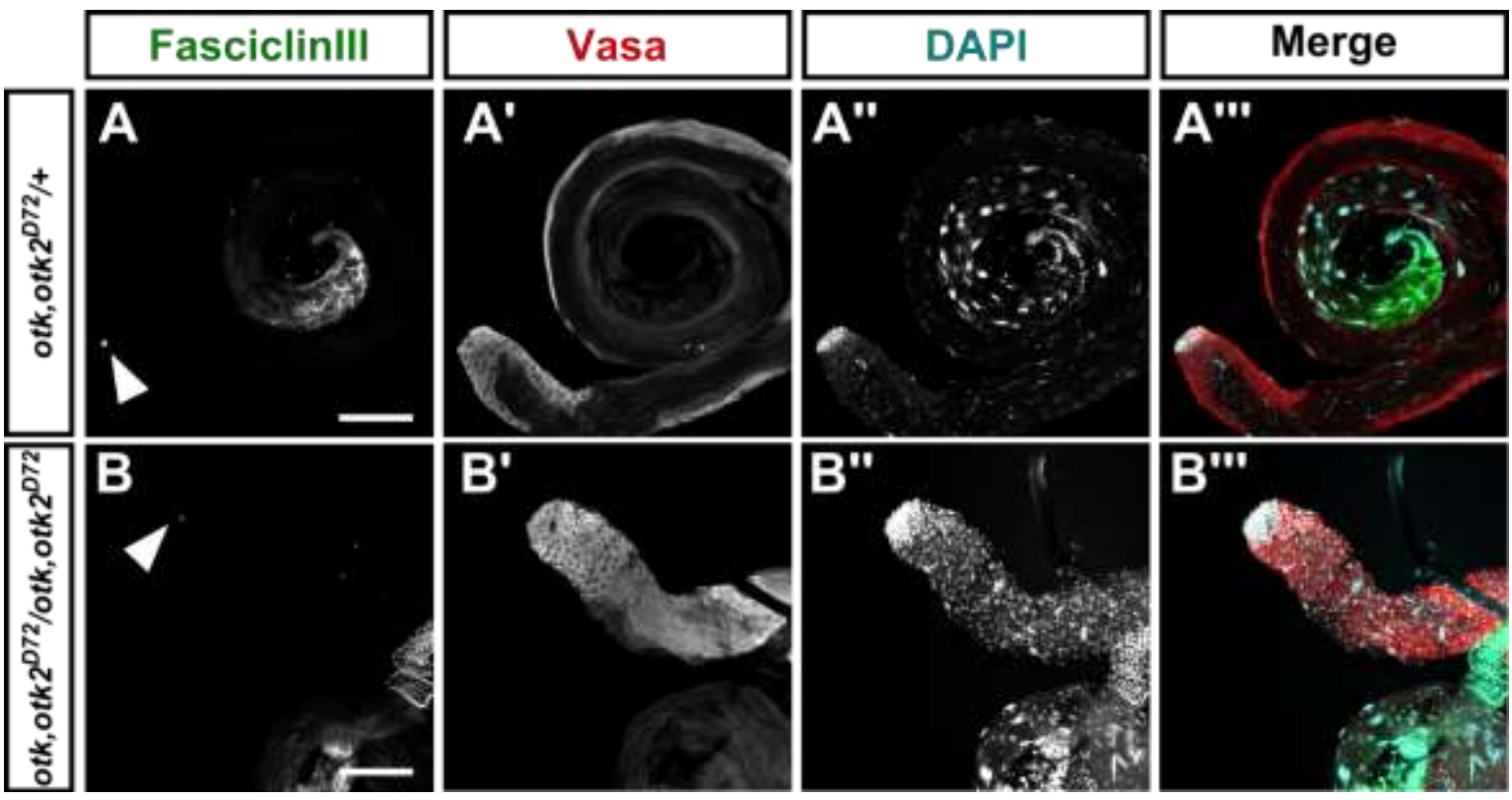

Fig. 36: Testes of otk,otk2 ${ }^{D 72}$ homozygous mutant males.

(A-C) Adult testes from males heterozygous (A) and homozygous (B) mutant for otk,otk2 ${ }^{D 72}$ were stained against FasciclinIII, which marks the hub (arrowhead in A and B) and terminal epithelium as well as Vasa, a marker for germline stem cells. Testis in (B) was unwound during the staining procedure. Scale bars: A-C $=100 \mu \mathrm{m}$.

To further confirm this, testes from males expressing a ProtamineB-eGFP (Raja \& Renkawitz-Pohl, 2005) transgene were analyzed. During Drosophila spermatogenesis, histones are replaced by protamines to achieve sufficient chromatin condensation (Raja \& Renkawitz-Pohl, 2005). Indeed, testes from males homozygous mutant for otk,otk2 ${ }^{D 72}$ contain all stages of development (Fig. 37Fig. 36 B, C), including late canoe stage (Fig. 36 B) and individualized spermatids with needleshaped nuclei (Fig. $36 \mathrm{C}$ ), corresponding to testes from heterozygous control males (Fig. 36 A). Live imaging of mature sperm revealed that mature sperm from homozygous mutant males is motile as well (data not shown). I conclude that any late defects in spermatogenesis or sperm motility are unlikely to account for the observed sterility of otk,otk2 $2^{D 72} /$ otk,otk2D72 adult males.

However, after crossing of homozygous mutant otk,otk2 ${ }^{D 72}$ males expressing ProtaminB-eGFP to white- females, no sperm could be detected in the female reproductive tract in contrast to the control group with heterozygous mutant males (Appendix 1), even though the mating behavior was not affected (Caroline Ripp, personal communication). This finding strongly suggests that male sterility of otk,otk2 $2^{D 72} /$ otk,otk2D72 animals is caused by a structural or mechanical defect of the male reproductive tract. 


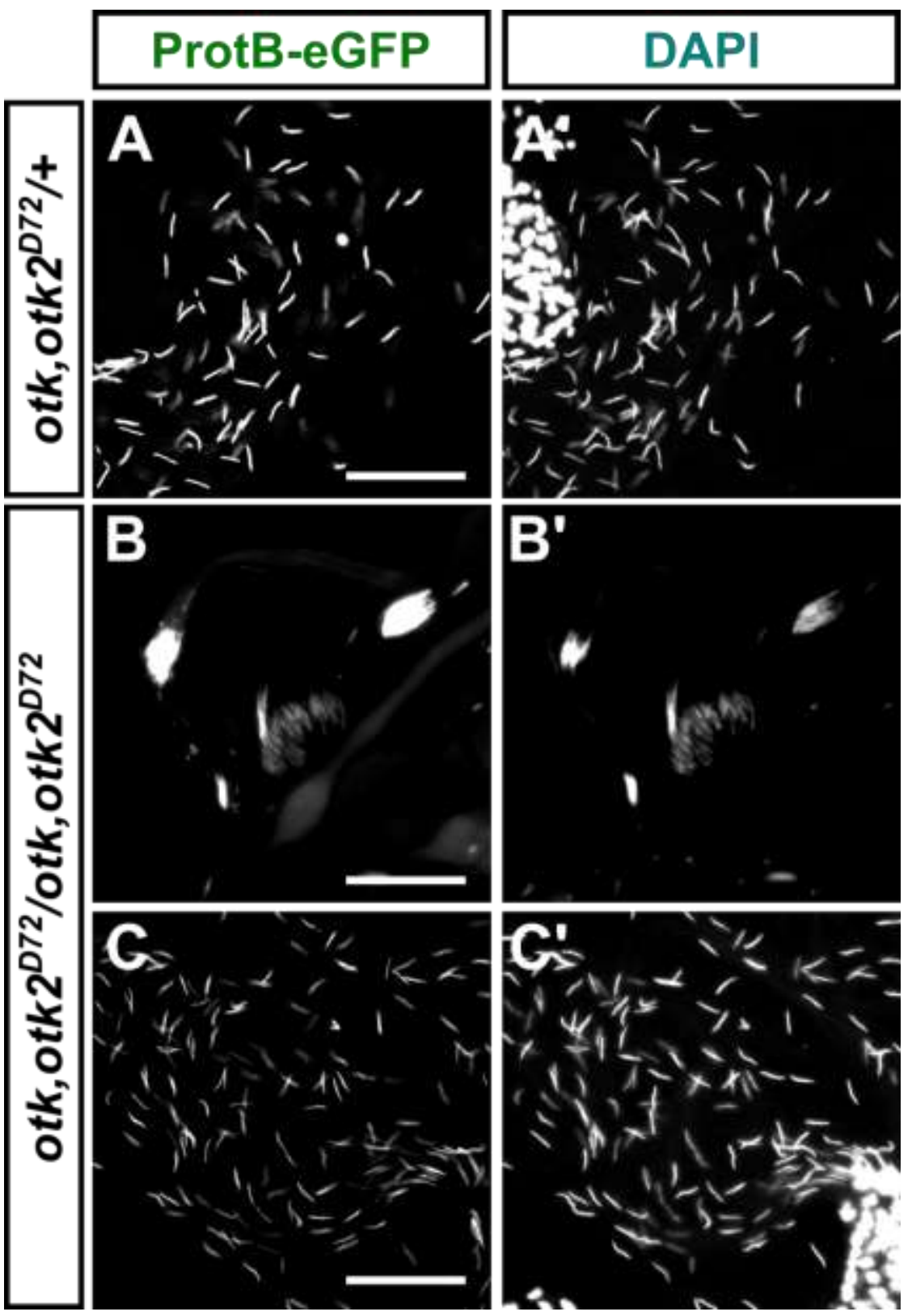

Fig. 37: Testes of otk,otk2 ${ }^{D 72}$ homozygous mutant males develop all stages of sperm development including motile sperm.

(A-C) Testes from adult males heterozygous (A) and homozygous $\mathbf{( B , C )}$ mutant for otk,otk2 ${ }^{D 72}$ carrying a ProtaminB-eGFP transgene. Images were taken by Caroline Ripp. Scale bars: A-C = $50 \mu \mathrm{m}$.

Wnt2 has been shown to be expressed in genital discs and to be involved in the attachment of the testes to the developing seminal vesicle as well as subsequent myoblast migration. Loss of wnt2 results in male sterility due to defects in male reproductive tract formation (Kozopas et al, 1998). Next, I therefore analyzed the expression of Otk and Otk2 in genital discs. Indeed, Otk as well as Otk2 are expressed in both female and male genital discs as determined by antibody stainings and reporter expression (Fig. 38). 

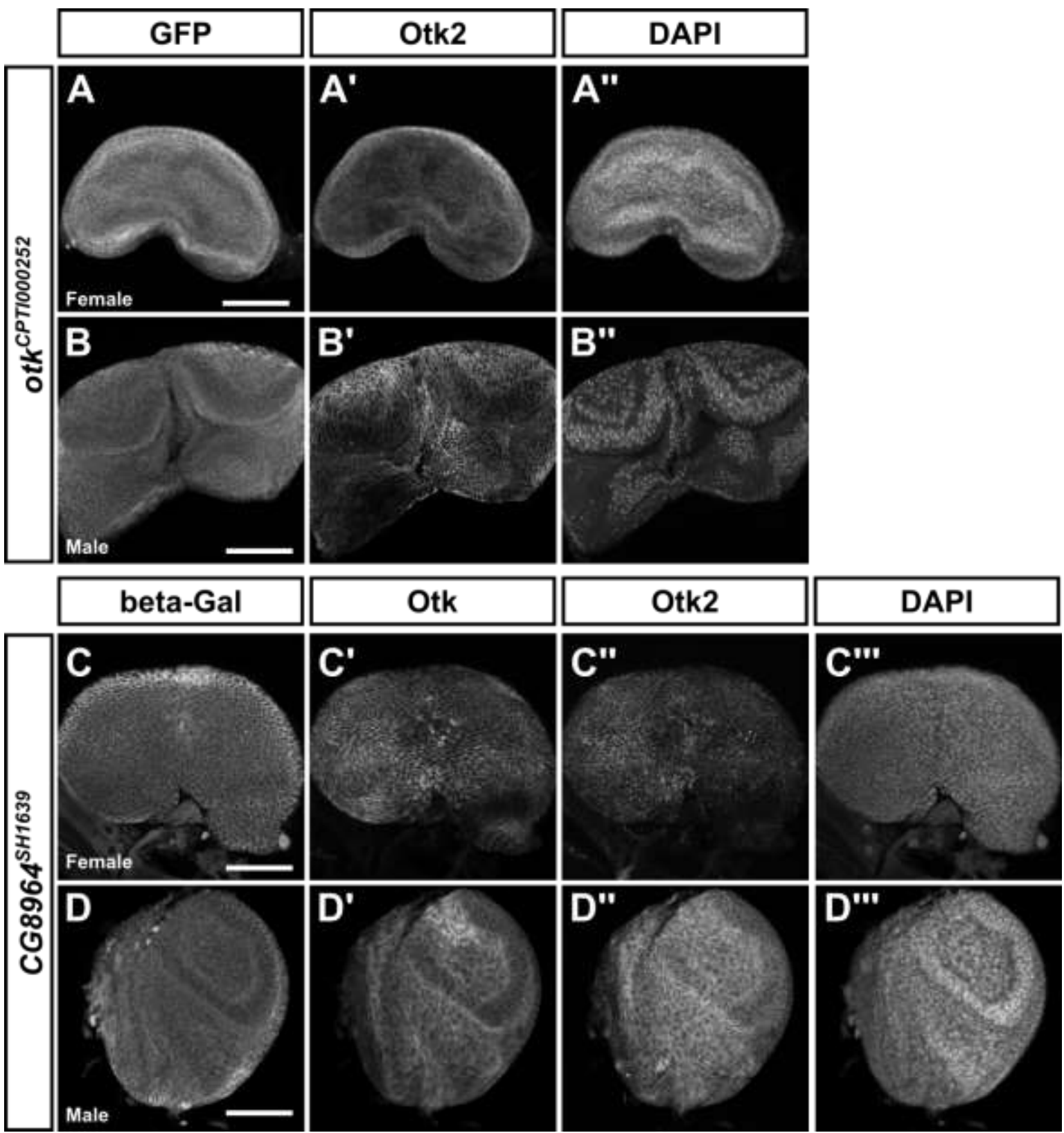

Fig. 38: Otk and 0tk2 are expressed in both male and female genital discs.

(A,B) Genital discs from transgenic flies expressing a GFP gene trap in the otk locus were stained against GFP, Otk2 and DAPI. Otk staining could not be detected, indicating that the line is an allele of otk. (C,D) Genital discs from transgenic flies expressing a lacZ gene trap in the otk2 locus were stained against beta-galactosidase, Otk, Otk2 and DAPI. Half of the male genital disc was lost during dissection in (D). Scale bar: $50 \mu \mathrm{m}$.

Since the testes of wht2 mutant males are not ensheathed by a continuous muscle layer (Kozopas et al, 1998), the muscle sheath of the male genital tract of otk,otk2 ${ }^{D 72}$ mutant males was analyzed. Surprisingly, the entire genital tract of otk,otk2 ${ }^{D 72}$ mutant males is surrounded by a complete muscle sheath (Fig. 39) and the filament organization of the single organs does not differ from that of heterozygous mutant control flies (data not shown). It was recently described that the seminal vesicle and 
the sperm pump contain multinucleated striated muscles, whereas the paragonia and ejaculatory duct are enclosed by mononucleated striated muscle fibers. In contrast, the testes are encircled by smooth muscle fibers (Susic-Jung et al, 2012). All of these types of muscle fibers could be identified (Fig. 39) and no difference between otk,otk2 ${ }^{D 72}$ homo- and heterozygous mutant males was observed.
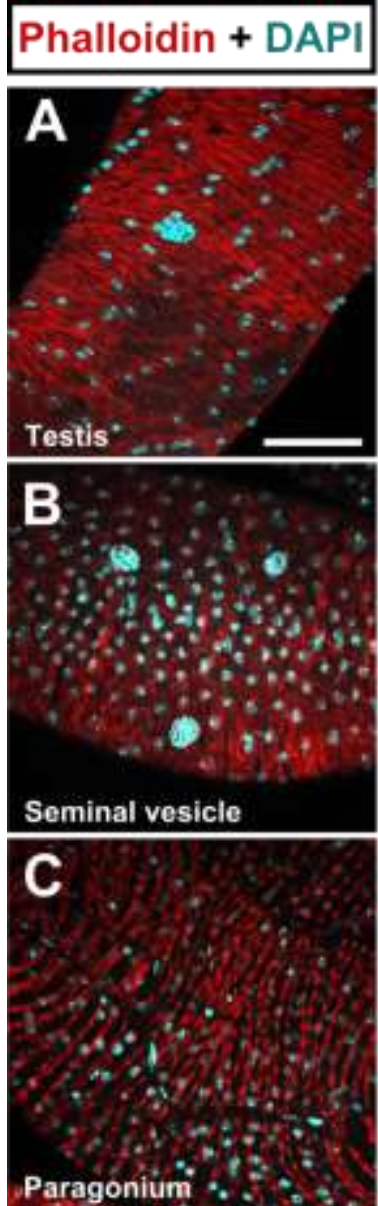
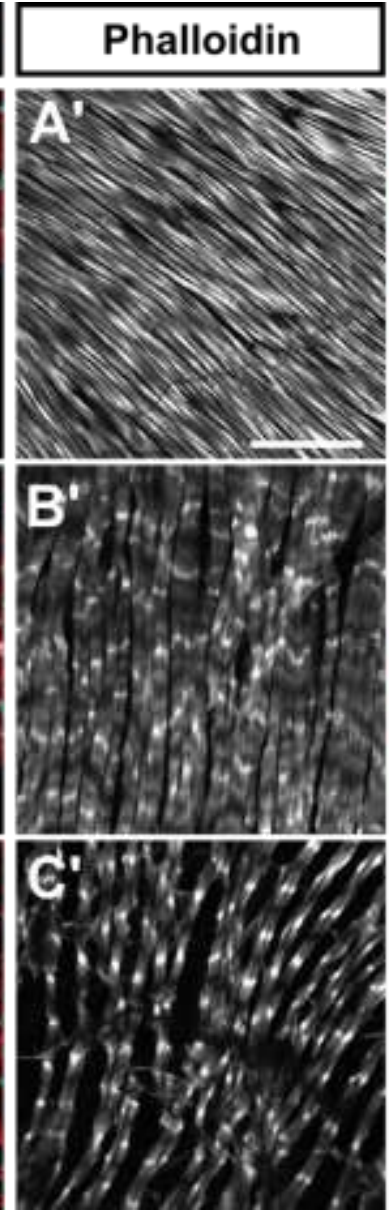
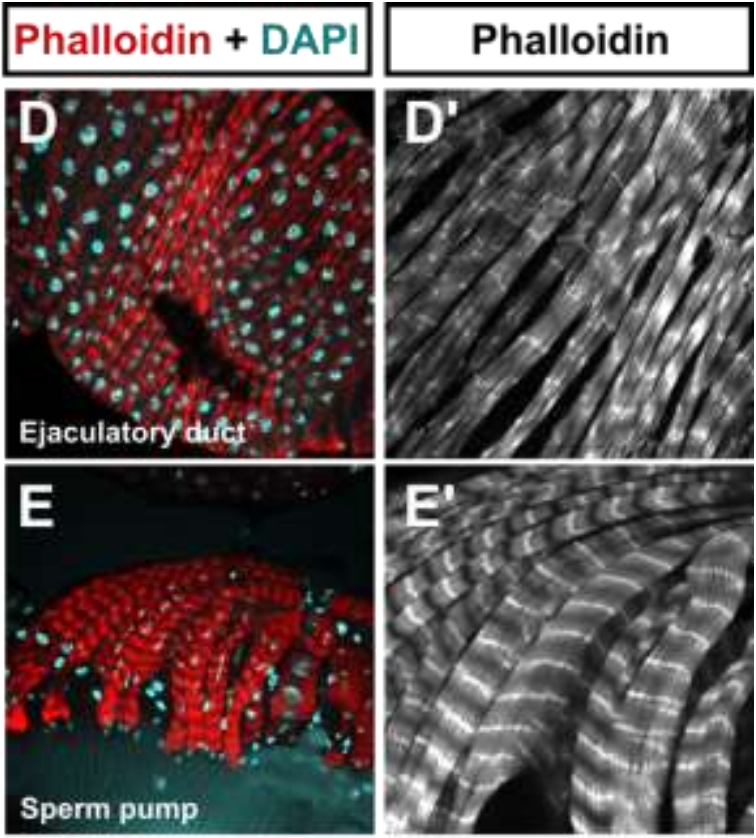

Fig. 39: The male reproductive system of homozygous otk,otk2 mutants is surrounded by striated and smooth muscles.

The adult muscle sheaths of the different organs of the male reproductive tract of otk,otk2 ${ }^{\text {D72 }}$ homozygous mutant males was visualized with Phalloidin for F-actin detection. As described in (SusicJung et al, 2012) the testis (A) is surrounded by smooth muscle, whereas the seminal vesicles (B), paragonia (C), ejaculatory duct (D) and sperm pump (E) are ensheathed by striated musculature. Scale bars: $A-E=50 \mu \mathrm{m} ; A^{\prime}-E^{\prime}=20 \mu \mathrm{m}$.

However, these analyzes revealed that the ejaculatory duct of homozygous mutant males is severely malformed. In contrast to the heterozygous control, the ejaculatory duct is much shorter and thickened (Fig. 40). The morphology of all the other organs of the reproductive tract is normal (Fig. 40). Further analysis of the muscle filaments of this organ uncovered that the ejaculatory duct indeed is severely obstructed (Fig. 
41). Until the obstruction the actin filament organization is normal, however, from the obstruction site on it is strongly disorganized (Fig. 41).
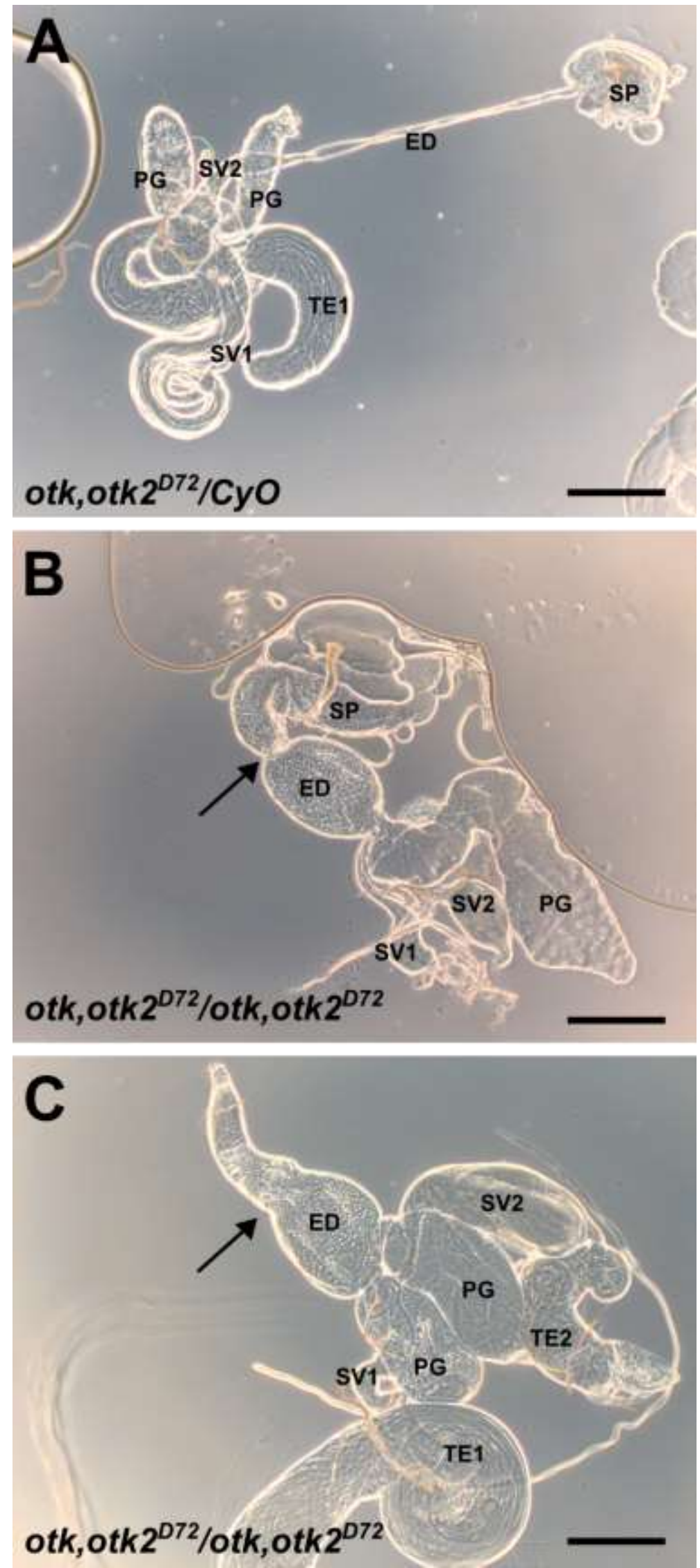

Fig. 40: otk,otk2 loss of function leads to changes in the morphology of the ejaculatory duct. 
Fig.40 (previous page): (A) Overview of the reproductive tract of males heterozygous for otk,otk2D72. One testis was lost during dissection. (B,C) Overview of the reproductive tract of males homozygous for otk,otk2 ${ }^{D 72}$. The arrow marks an obstruction in the ejaculatory duct, which is much shortened compared to (A). In (B) both testes were lost during dissection and in (C) the sperm pump was lost during dissection. ED, ejaculatory duct; PG, paragonium (accessory gland); SP, sperm pump; SV, seminal vesicle; TE, testis. Scale bars: $200 \mu \mathrm{m}$.
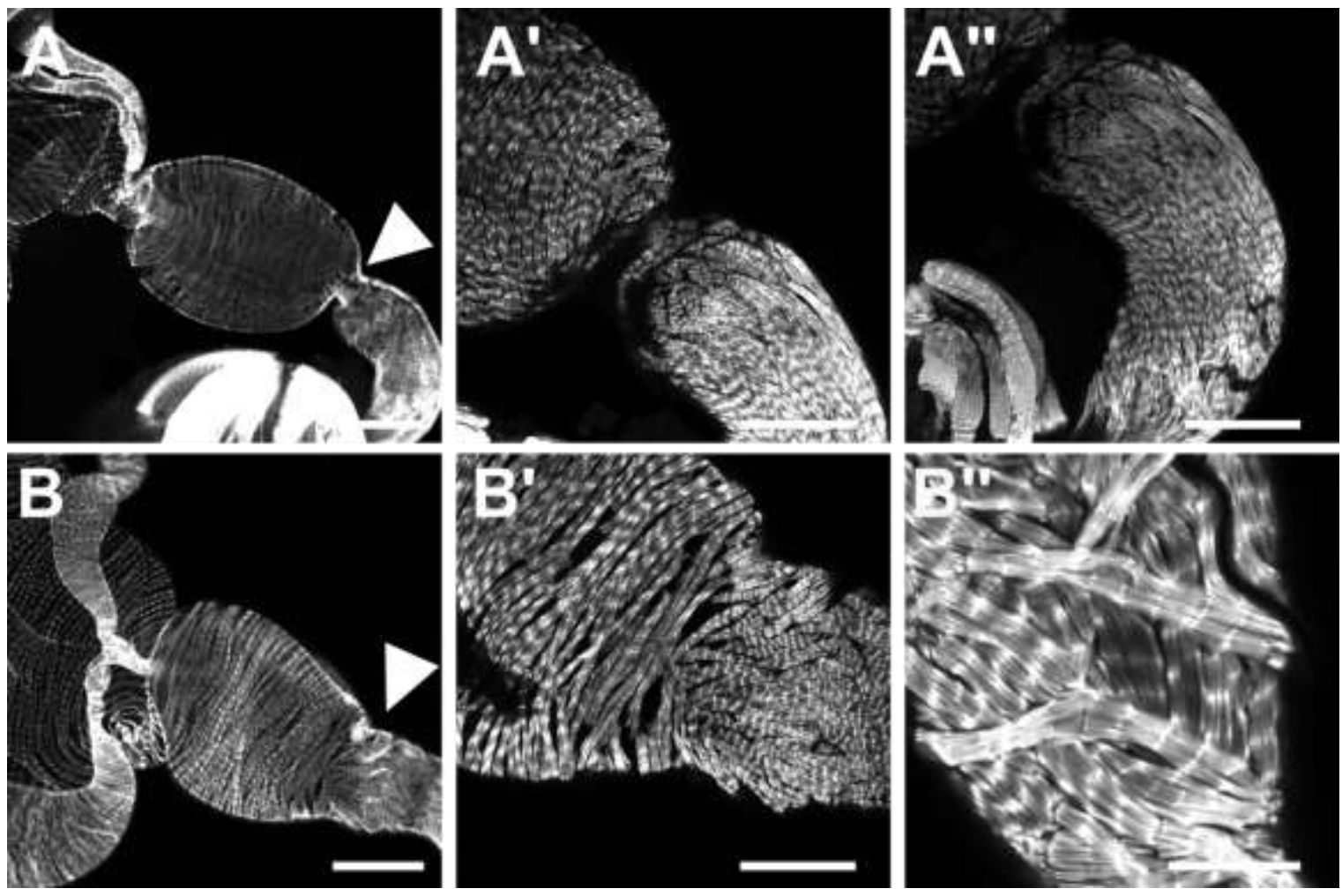

Fig. 41: Ejaculatory duct obstruction in otk,otk2 homozygous mutant males.

The muscle sheath of the adult ejaculatory duct of otk,otk2 $2^{D 72}$ homozygous mutant males was visualized with Phalloidin for F-actin detection. (A) represents an enlarged view of the ejaculatory duct in Fig. 40 B and (B) of the one in Fig. 40 C. $\mathbf{A}^{\prime}$ and $\mathbf{A}^{\prime \prime}$ as well as B' and B' are higher magnifications of the obstruction marked with an arrowhead in A and B, respectively. Scale bars: A,B = $100 \mu \mathrm{m} ; A^{\prime}, B^{\prime}, B^{\prime \prime}$ $=50 \mu \mathrm{m} ; \mathrm{A}^{\prime \prime}=20 \mu \mathrm{m}$.

\section{7 otk and otk2 interact with the Wingless receptor Frizzled}

In Drosophila, Frizzled1 (Fz) and Frizzled2 (Dfz2) are functionally redundant receptors for Wingless, activating canonical signaling. Indeed, loss of both $f z$ and $D f z 2$ results in a phenotype that is virtually indistinguishable from wingless mutants (Bhat, 1998; Bhanot et al, 1999 ; Chen \& Struhl, 1999; Müller et al, 1999). Additionally, Fz non-redundantly controls planar cell polarity signaling in Drosophila (Krasnow \& Adler, 1994; Adler et al, 1997). In contrast, Dfz2 does not display any polarity signaling capacity and is most likely solely involved in canonical signaling. Its ligand 
binding domain has a 10-fold higher affinity for Wg than the cysteine-rich domain of Fz (Zhang \& Carthew, 1998; Chen \& Struhl, 1999; Rulifson et al, 2000). I challenged the hypothesis that Otk and Otk2 could act as co-receptors for either Fz or Dfz2 by testing for both genetic and biochemical interactions.

Indeed, epistasis studies using different $f z$ alleles indicated that otk,otk2 genetically interact, however, there were differences with regard to the combinations of $f z$ alleles used (Table 18).

Table 18: otk,otk2 genetically interacts with $f z$

\begin{tabular}{|c|c|}
\hline otk $^{\mathrm{A} 1} /$ otk $^{\mathrm{A} 1}$ & viable \\
\hline otk2 $2^{\mathrm{c} 26} /$ otk2 $2^{\mathrm{C} 26}$ & viable \\
\hline otk,otk2 $^{\mathrm{D} 72} /$ otk,otk2 ${ }^{\mathrm{D} 72}$ & viable (male sterile) \\
\hline $\mathrm{fz}^{\mathrm{J22}} / \mathrm{fz}^{\mathrm{J22}}$ & viable with PCP defects \\
\hline $\mathrm{fz}^{\mathrm{H} 51} / \mathrm{fz}^{\mathrm{H} 51}$ & viable with PCP defects \\
\hline $\mathrm{fz}^{\mathrm{R52}} / \mathrm{fz}^{\mathrm{R52}}$ & lethal \\
\hline $\mathrm{fz}^{\mathrm{P21}} / \mathrm{fz}^{\mathrm{P} 21}$ & lethal \\
\hline $\mathrm{fz}^{\mathrm{R} 52} / \mathrm{fz}^{\mathrm{J} 22}$ & viable with PCP defects \\
\hline $\mathrm{fz}^{\mathrm{R} 52} / \mathrm{fz}^{\mathrm{P21}}$ & viable with PCP defects \\
\hline $\mathrm{fz}^{\mathrm{R} 52} / \mathrm{fz}^{\mathrm{H} 51}$ & viable with PCP defects \\
\hline $\mathrm{fz}^{\mathrm{J22}} / \mathrm{fz}^{\mathrm{P} 21}$ & viable with PCP defects \\
\hline $\mathrm{fz}^{\mathrm{J} 22} / \mathrm{fz}^{\mathrm{H} 51}$ & viable with PCP defects \\
\hline $\mathrm{fz}^{\mathrm{H} 51} / \mathrm{fz}^{\mathrm{P} 21}$ & viable with PCP defects \\
\hline otk $^{\mathrm{A} 1} / \mathrm{otk}^{\mathrm{A} 1} ; \mathrm{fz}^{\mathrm{J} 22} / \mathrm{fz}^{\mathrm{J} 22}$ & viable with PCP defects \\
\hline otk2 $2^{\mathrm{C} 26} /$ otk $^{\mathrm{c26}} ; \mathrm{fz}^{\mathrm{J} 22} / \mathrm{fz}^{\mathrm{J} 22}$ & viable with PCP defects \\
\hline otk,otk2 $2^{\mathrm{D} 72} /+; \mathrm{fz}^{\mathrm{J} 22} / \mathrm{fz}^{\mathrm{J22}}$ & viable with PCP defects \\
\hline otk,otk2 ${ }^{\mathrm{D} 72} /$ otk,otk2 $2^{\mathrm{D} 72} ; \mathrm{fz}^{\mathrm{J} 22} /+$ & viable \\
\hline otk,otk2 $^{\mathrm{D} 72} /$ otk,otk2 ${ }^{\mathrm{D} 72} ; \mathrm{fz}^{\mathrm{J22}} / \mathrm{fz}^{\mathrm{J22}}$ (zygotic) & lethal \\
\hline otk,otk2 $^{\mathrm{D72}} /$ otk,otk2 ${ }^{\mathrm{D} 72} ; \mathrm{fz}^{\mathrm{H} 1} / \mathrm{fz}^{\mathrm{H51}}$ (zygotic) & lethal \\
\hline otk,otk2 ${ }^{\mathrm{D} 2} /$ otk,otk2 ${ }^{\mathrm{D} 72} ; \mathrm{fz}^{\mathrm{R} 52} / \mathrm{fz}^{\mathrm{J} 22}$ (zygotic) & viable with PCP defects \\
\hline otk,otk2 $^{\mathrm{D} 72} /$ otk,otk2 $2^{\mathrm{D} 72} ; \mathrm{fz}^{\mathrm{R52}} / \mathrm{fz}^{\mathrm{P} 21}$ (zygotic) & viable with PCP defects \\
\hline otk,otk2 ${ }^{\mathrm{D} 72} /$ otk,otk2 ${ }^{\mathrm{D} 72} ; \mathrm{fz}^{\mathrm{RS2}} / \mathrm{fz}^{\mathrm{H} 51}$ (zygotic) & viable with PCP defects \\
\hline otk,otk2 ${ }^{\mathrm{D} 72} /$ otk,otk2 ${ }^{\mathrm{D} 72} ; \mathrm{fz}^{\mathrm{J22}} / \mathrm{fz}^{\mathrm{P} 21}$ (zygotic) & viable with PCP defects \\
\hline otk,otk2 $^{\mathrm{D} 72} /$ otk,otk2 $2^{\mathrm{D} 72} ; \mathrm{fz}^{\mathrm{J22}} / \mathrm{fz}^{\mathrm{H} 51}$ (zygotic) & lethal \\
\hline otk,otk2 $^{\mathrm{D} 72} /$ otk,otk2 $2^{\mathrm{D} 72} ; \mathrm{fz}^{\mathrm{H51}} / \mathrm{fz}^{\mathrm{P} 21}$ (zygotic) & viable with PCP defects \\
\hline otk,otk $2^{\mathrm{D} 72} /$ otk,otk2 ${ }^{\mathrm{D} 72} ; \mathrm{fz}^{\mathrm{RS2}} / \mathrm{fz}^{\mathrm{J22}}$ (maternal) & viable with PCP defects \\
\hline otk,otk $2^{\mathrm{D} 72} /$ otk,otk2 ${ }^{\mathrm{D} 72} ; \mathrm{fz}^{\mathrm{RS2}} / \mathrm{fz}^{\mathrm{P} 21}$ (maternal) & lethal \\
\hline otk,otk2 $^{\mathrm{D} 72} /$ otk, otk2 ${ }^{\mathrm{D} 72} ; \mathrm{fz}^{\mathrm{R52}} / \mathrm{fz}^{\mathrm{H} 51}$ (maternal) & lethal \\
\hline otk,otk $2^{\mathrm{D} 72} /$ otk,otk2 ${ }^{\mathrm{D} 72} ; \mathrm{fz}^{\mathrm{j22}} / \mathrm{fz}^{\mathrm{P} 21}$ (maternal) & viable with PCP defects \\
\hline otk,otk2 ${ }^{\mathrm{D} 72} /$ otk,otk2 ${ }^{\mathrm{D} 72} ; \mathrm{fz}^{\mathrm{J22}} / \mathrm{fz}^{\mathrm{H} 1 \mathrm{1} 1}$ (maternal) & lethal \\
\hline otk,otk $2^{\mathrm{D} 72} /$ otk,otk2 $2^{\mathrm{D} 72} ; \mathrm{fz}^{\mathrm{H}{ }^{\mathrm{H1}}} / \mathrm{fz}^{\mathrm{P} 21}$ (maternal) & lethal \\
\hline $\operatorname{otk}^{\mathrm{A} 1} /$ otk $^{\mathrm{A} 1} ; \mathrm{fz}^{\mathrm{R} 52}, \mathrm{Df}(3 \mathrm{~L}) \mathrm{DFz2} /+$ & viable \\
\hline otk2 ${ }^{\mathrm{C} 26} /$ otk2 ${ }^{\mathrm{C} 26} ; \mathrm{fz}^{\mathrm{R} 52}, \mathrm{Df}(3 \mathrm{~L}) \mathrm{DFz} 2 /+$ & viable \\
\hline otk,otk2 $^{\mathrm{D} 72} /$ otk,otk2 ${ }^{\mathrm{D} 72} ; \mathrm{fz}^{\mathrm{R} 52}, \mathrm{Df}(3 \mathrm{~L}) \mathrm{Dfz2} /+$ & viable \\
\hline otk,otk2 ${ }^{\mathrm{D} 72} /$ otk,otk2 ${ }^{\mathrm{D} 72} ; \mathrm{fz}^{\mathrm{R} 52}, \mathrm{Df}(3 \mathrm{~L}) \mathrm{Dfz} 2 / \mathrm{fz}^{\mathrm{R} 52}, \mathrm{Df}(3 \mathrm{~L}) \mathrm{Dfz2}$ & lethal \\
\hline
\end{tabular}


To verify the observed genetic interactions biochemically, GFP-tagged Otk and Otk2 and Myc-tagged Fz1 constructs under the control of an Actin promoter were cooverexpressed in S2r+ cells. Cell lysates were subjected to anti-Myc IP. Immunoblotting with anti-GFP antibody demonstrated that Otk-GFP as well as Otk2GFP could be co-precipitated with Fz1-Myc (Fig. 42). The interaction between OtkGFP and Fz1-Myc seemed to be quite weak, resulting in a rather faint band for OtkGFP, however this was observed in several experiments (Fig. 42).

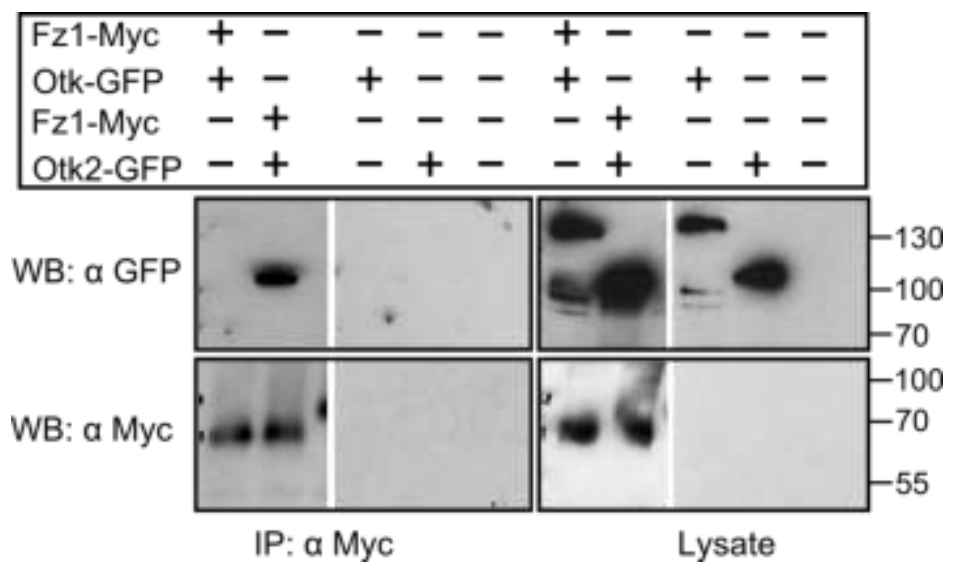

Fig. 42: Off-track and Off-track2 interact with Frizzled1.

Otk-GFP or Otk2-GFP and Fz1-Myc expression vectors were transfected as indicated in Drosophila S2r+ cells. Cell lysates were immunoprecipitated and analyzed by Western Blot with the indicated antibodies. IP, Immunoprecipitation; WB, Western Blot. 


\section{DISCUSSION}

Despite their key role, Frizzled proteins are far from being the only receptors controlling Wnt signaling. The role of additional co-receptors and their emerging influence on signaling outcome has been appreciated within the past decade. Vertebrate PTK7 was described as a novel Wnt co-receptor that confers signaling specificity in response to different Wnt ligands. In this work, I characterized the Drosophila PTK7 homologs Off-track (Otk) and Off-track2 (Otk2) with regard to signaling mediated by different Wnt ligands present in Drosophila.

\subsection{Expression of Otk and Otk2}

Both RNA and protein expression analyses revealed that Otk and Otk2 are dynamically expressed during embryonic as well as larval development. Since only little is known about the precise role of Drosophila Otk so far, these analyses might give valuable hints regarding its function.

\subsubsection{Otk and Otk2 co-localize with Wingless}

During Drosophila embryonic development, Otk and Otk2 are expressed in segmental stripes that overlap with the position of Wingless (Wg) stripes (see 3.3.2, 3.3.3). This could mean that Otk and Otk2 are receptors for Wg in early embryonic development or that they are Wg targets. Since Otk expression is rather weak in early embryonic development and can only be detected from stage 9/10 on, whereas Wg expression is detectable much earlier, the latter possibility seems more likely. However, localization of Otk in segmental stripes was not changed in embryos homozygous mutant for $w g$.

Furthermore, Otk and Otk2 were found to be co-expressed with Wg in the visceral mesoderm (see 3.3.3). Wg expression in parasegment 8 of the visceral mesoderm as well as the developing fore- and hindgut was shown to be required for the formation of the second midgut constriction (Immerglück et al, 1990). The finding that Otk expression is broader than the region of Wg expression might be explained by the fact that $\mathrm{Wg}$ acts as a morphogen and induces expression of Otk over a distance. Apart from Wg, Wnt4 is expressed in the visceral mesoderm and developing gut as well. 
Wnt4 transcripts were detected in the foregut, hindgut and two locations in the visceral mesoderm of the midgut, which correspond to the gastric caeca and the second midgut constriction (Graba et al, 1995). Formation of midgut constrictions was not affected in otk and otk,otk2 mutants, indicating that otk and otk2 most likely do not play a role in this process (data not shown). Furthermore, the localization of Otk in segmental stripes was not changed in embryos homozygous mutant for $w g$ and $w n t 4$, indicating that Otk expression most likely is not regulated by Wg or Wnt4.

A ChIP-on-chip approach aiming at elucidating the transcriptional network regulating smooth muscle development identified the otk enhancer to be a target of the transcription factor Biniou (homolog of vertebrate FoxF) in trunk visceral smooth muscle development (Jakobsen et al, 2007). In biniou mutant embryos, the visceral mesoderm expression of Otk was abolished, whereas expression in other regions of the embryo was not affected. Similarly, mouse PTK7 expression within the primitive streak was shown to depend on FoxF1 (Jakobsen et al, 2007). However, no direct connection between Biniou function and Wnt signaling, which might explain the peculiar expression pattern in the gut, was described so far.

\subsubsection{Otk and Otk2 co-localize with Derailed}

Otk is expressed within the Drosophila embryonic nerve chord, but shows enrichment in those axon tracts that cross the anterior commissure (AC). Interestingly, the Derailed (Drl) receptor tyrosine kinase is expressed exclusively by neurons that project in the anterior commissure. Drl was shown to be expressed in growth cones and axons of neurons as they enter the AC and extend through it, but it gets rapidly down regulated when they leave the AC (Callahan et al, 1995; Bonkowsky et al, 1999). Wnt5 on the other hand is expressed along the posterior commissure, where it acts as a repellent ligand for Derailed expressing neurons that cross the midline along the anterior commissure (Yoshikawa et al, 2003; Fradkin et al, 2004). Possibly, Otk/Otk2 and Drl interact in Wnt5-repulsive axon guidance along the AC.

\subsubsection{Otk expression depends on Wnt2}

To determine whether Otk expression might be controlled by Wnt signaling, I compared Otk expression in different wht mutant backgrounds (3.4). Interestingly, Otk does not seem to be regulated by Wingless or Wnt4. Both the segmental localization in early embryos as well as the localization in the developing gut and 
nervous system was not affected. However, the expression of Otk in the nervous system appeared to be slightly reduced in wht5 mutant embryos. Interestingly, overall Otk expression levels were strongly reduced in embryos homozygous mutant for wht2. In particular, segmental stripe expression starting in stage 9/10 was abolished and expression in the developing gut as well as the nervous system was still detectable, but strongly reduced compared to heterozygous control embryos. This indicates that Otk is a target of Wnt2 signaling and that Wnt2 is required to maintain high Otk expression levels.

\section{2 otk loss of function does not lead to lethality}

Flies homozygous mutant for otk ${ }^{A 1}$ were homozygous viable and did not show any phenotypic defects related to PCP or canonical signaling (see 3.6). This strongly disagrees with reports on the previously generated homozygous lethal ot ${ }^{3}$ allele (Winberg et al, 2001; Cafferty et al, 2004; Peradziryi et al, 2011). Since I have unambiguously shown that the complete otk locus is deleted in the otk ${ }^{A 1}$ allele, this can only mean that the reported lethality of $o t k^{3}$ was due to a second site hit on the same chromosome and was not caused by mutation of otk.

Embryos mutant for ot $^{3}$ were reported to display axon guidance defects in the CNS, but no data supporting this statement was shown in the respective publication (Winberg et al, 2001). In addition, the projection of SNa motor neurons to their muscle targets was demonstrated to be disturbed in $56 \%$ of ot $k^{3}$ homozygous mutants as assayed by Fasciclin II staining of motor projections. However, the underlying data seems rather weak since the representative image of an ot $k^{3}$ mutant embryo was taken at a different focal plane compared to the wild type control. Moreover, the authors claimed to have obtained similar defects upon Gal4-driven expression of short antisense Otk transcripts. Inconclusively, the UAS-containing gene-regulatory P-element utilized for this analysis is located even upstream of the otk 5'UTR (Winberg et al, 2001) and can therefore impossibly mediate the effects described.

Unfortunately, the finding that CNS longitudinal axon tracts display breaks and defasciculation defects in $15 \%$ of ot $k^{3}$ mutant embryos was not further commented on (Winberg et al, 2001). A similar phenotype was reported for wht5 mutant embryos 
(Fradkin et al, 2004) and might hint at a possible connection between Otk and Wnt5/Drl signaling.

Furthermore, I tried to reproduce whether the otk ${ }^{A 1}$ homozygous mutant animals display the photoreceptor targeting defects reported by another study (Cafferty et al., 2004). The ommatidia or single eye units in the adult Drosophila visual system each contain eight photoreceptor cells (R1-R8), which project into the optic lobes. Whereas R1-R6 cells connect to the lamina, R7 and R8 cells project to the medulla. In this study, Otk was shown to predominantly localize to the growth cones of R1-R6 photoreceptor axons and to be required for lamina-specific targeting of these axons. This conclusion was drawn from the observation that in otk ${ }^{3}$ mosaic animals expressing the R2-R5 marker rough- $\tau$-lacZ, about one third of R2-R5 axons projected aberrantly into the medulla, instead of terminating in the lamina (Cafferty et al, 2004). Unfortunately, the origin of the reporter line was not referenced in the publication. However, inspection of a rough- $\tau$-lacZ reporter line obtained from Christian Klämbt revealed that the wild-type reporter line showed the same phenotype as the ot $^{3}$ mutants in (Cafferty et al, 2004) (data not shown). Therefore, the expression of rough- $\tau$-lacZ in otk ${ }^{A 1}$ mutant animals was not further analyzed.

A recent study reported a role for Otk in embryonic patterning by interaction with Wnt4 (Peradziryi et al, 2011). As described in 1.4.1and 1.4.3, patterning of the embryonic epidermis is mediated by segment polarity genes like wingless. Wingless is expressed at the posterior of each segment and specifies naked cuticle fate, whereas anterior cells express Engrailed and secrete denticles. Wnt4 has the capacity to oppose Wingless signaling in the ventral epidermis since ubiquitous overexpression of Wnt4 phenocopies loss of $w g$ activity (Gieseler et al, 1999). However, wnt4 loss of function does not influence embryonic patterning since homozygous mutant embryos hatch without obvious defects and die during larval stage (Cohen et al, 2002). ot ${ }^{3}$ mutant embryos published by (Winberg et al, 2001) were reported to have narrowed denticle belts and, conversely, overexpression of Otk led to ectopic denticles (Peradziryi et al, 2011). I could not reproduce any of these phenotypes me as neither otk ${ }^{A 1}$ nor otk,otk2 ${ }^{D 72}$ loss of function produced any cuticular defects (data not shown). Ubiquitous overexpression of one copy of full length Otk both under control of UASp (drives expression in the germline and somatic cells, this work) and UASt (drives expression in somatic cells, Winberg et al, 2001) resulted in viable flies, which did not display any obvious embryonic defects (data not shown). Strikingly, the previously 
published conclusions were based on zoomed images of single segments and were not supported by any quantification (Peradziryi et al, 2011). Based on the finding that co-overexpression of Otk and Wnt4 leads to ectopic denticles, the authors concluded that Otk acts as a receptor for Wnt4 (Peradziryi et al, 2011). Yet, the same phenotype is already achieved by overexpression of Wnt4 alone and therefore might be mediated by any other receptor endogenously expressed in Drosophila embryos (Gieseler et al, 1999). Similarly, the destabilization of cytoplasmic Armadillo, which was ascribed to co-overexpression of Otk and Wnt4 (Peradziryi et al, 2011), has been previously described to be mediated by overexpression of Wnt4 alone (Gieseler et al, 1999). Moreover, loss of otk was shown to block the activity of overexpressed Wnt4, however, any quantification supporting this finding is lacking (Peradziryi et al, 2011). Finally, the authors claim that overexpression of Otk in wings leads to both (canonical) wing margin and planar cell polarity defects at the same time (Peradziryi et al, 2011) - such a phenotype cannot be explained by any existing signaling pathway model and could not be reproduced either (data not shown).

In conclusion, the misleading findings presented by Winberg, Cafferty and Peradziryi et al. did not contribute to elucidating the function of Drosophila off-track.

\subsection{Drosophila Otks form homo- and heterodimers}

Biochemical studies revealed that Otk and Otk2 can form homo- as well as heterodimers upon transient expression in S2r+cells and that this interaction is most likely dependent on the transmembrane domain (see 3.2).

Homodimerization has been described for Xenopus PTK7 (Annette Borchers, personal communication) as well as for Drosophila Derailed (Jasprien Noordermeer, personal communication). Differential interaction of receptor subclasses might be a way to confer signaling pathway specificity. In this context, Drosophila Ryks similar to Otks were shown to form heterodimers with each other and interaction in both receptor classes seems to depend on the transmembrane domain (Jasprien Noordermeer, personal communication). It will be interesting to determine whether different Wnts are able to inhibit or increase dimerization and if this mechanism is required to recruit additional receptors to the membrane. 


\subsection{Otk and Otk2 might function as (co-)receptors in different Wnt signaling pathways}

\subsubsection{Possible role of Otk/Otk2 in Wnt2 signaling during male reproductive tract formation}

Males homozygous mutant for otk,otk2 are sterile. However, the morphology of testes including the germ cells and somatic cell types that support spermatogenesis are normal, suggesting that sterility is caused by structural and mechanical defects of the reproductive tract. This was supported by the finding that sperm from homozygous mutant males cannot enter the female reproductive tract even though no obvious changes in mating behaviour could be observed (Caroline Ripp, personal communication).

In Drosophila, the embryonic gonads give rise to the testes, whereas the genital disc gives rise to all other external genitalia and internal structures that connect to the testis, i.e. accessory glands, anal plate, ejaculatory duct and seminal vesicle (Estrada et al, 2003). During metamorphosis, the gonads and the somatic parts of the reproductive tract grow towards each other and finally join (Kozopas et al, 1998; Nanda et al, 2009). The testes, in which all stages of spermatogenesis take place, are surrounded by a basal membrane, a muscle layer and outermost pigment cells. Both outer pigment cells and the inner muscle layer are disturbed in Wnt2 mutants (Kozopas et al, 1998), leading to male sterility due to improper testis morphogenesis. Since the results obtained for otk,otk2 sterile males were very reminiscent of what is published for Drosophila Wnt2, I analyzed the sheath surrounding the male reproductive tract for the presence of pigment cells and the formation of the muscle sheath. In contrast to Wnt2 mutants, which completely lack pigment cells, the specification of these cells was not disturbed in otk,otk2 homozygous mutant males. This can be explained by the recent finding that Wnt2 is expressed male-specifically in the somatic embryonic gonad and is necessary and sufficient for pigment cell precursor formation in the embryo (DeFalco et al, 2008b). In contrast, Otk expression could neither be detected in the embryonic somatic gonad nor the germ cells (data not shown) and is therefore unlikely to be involved in pigment cell formation.

Only little is known about the development and assembly of the muscle sheaths of the Drosophila male reproductive tract. Twist-expressing cells are associated with the genital disc and presumably are myoblast precursors. In wht2 mutant testes, myosin- 
expressing tissue is present, even though covering the testes incompletely (Kozopas et al, 1998). This indicates that Wnt2 is most likely not required for muscle specification. Instead, Wnt2 seems to be involved in the attachment of the testes and the developing seminal vesicle. It was suggested that these muscle precursors migrate over the seminal vesicle onto the testis to form the muscle sheath and that Wnt2 might affect their migration or adherence (Kozopas et al, 1998). Migration defects in wht2 mutants might not be attributable to a loss of motility, but rather to a loss of the ability of cells to determine their final position. The entire genital tract of male sterile otk,otk2 mutant males is covered by a continuous muscle layer as determined by Phalloidin staining of actin filaments (3.6.4, Fig. 39), indicating that general muscle migration and attachment does not seem to be affected. However, the ejaculatory duct of these males is constricted and this obstruction most likely leads to the observed sterility. It is intriguing to re-investigate the phenotype of wnt2 homozygous mutant males with regards to these findings.

Another study reported a requirement for Wnt2 for the correct patterning of direct flight muscles (DFMs), explaining the phenotype of held-out wings in wht2 mutant flies (Kozopas et al, 1998; Kozopas \& Nusse, 2002). Such a phenotype could not be observed for otk or otk,otk2 mutant flies, even when raised at higher temperatures (data not shown). Therefore it seems unlikely that Otk interacts with Wnt2 in DFM patterning.

Furthermore, Wnt2 was demonstrated to function in tracheal development together with Wingless (Llimargas \& Lawrence, 2001). But since Otk is not expressed in the tracheal system, this signaling pathway was not further studied.

Interestingly, it should be mentioned in this context that wht4 loss of function also leads to female and male sterility. In wnt4 mutant females the ovariolar sheath fails to migrate properly over the developing ovarioles. Wnt4 regulates cell movement during ovarian organogenesis by interaction with Dishevelled and Frizzled 2 (Dfz2), which was postulated to be the primary Wnt4 receptor in this process (Cohen et al, 2002). This was based on the observations that transheterozygous $D f z 2^{C 2} / D f z 2^{C 1}$ animals are sterile both as males and females (Chen \& Struhl, 1999) and displayed the identical phenotype of folded ovarioles (Cohen et al, 2002). Dfz2-Dsh-Wnt4 signaling was also reported to regulate retinal axon guidance in the visual system (Sato et al, 2006). Since strong ubiquitous overexpression of UASp-Otk-GFP leads to female 
sterility (data not shown) it will be interesting to investigate whether Otk might function in Dfz2-Wnt4 signaling as well.

\subsubsection{Possible role of Otk/Otk2 in Wnt5/Drl signaling}

Co-expression of Drl and Otk along the anterior commissure (3.3.3, Fig. 28, see also 4.1.2) might hint at a potential interaction between these receptor tyrosine kinases, which will have to be further characterized genetically and biochemically. Interestingly, both wnt5 and $d r l$ mutants display subtle nervous system defects, but are homozygous viable (Dura et al, 1993; Callahan et al, 1995; Yoshikawa et al, 2003; Fradkin et al, 2004). This could be explained by the abundance of Wnt5 receptors and co-receptors acting in a redundant manner with Drl and one such candidate might be Otk. Preliminary experiments indicate that Otk/Otk2 enrichment at anterior commissures is abolished in drl mutant embryos (data not shown), supporting the hypothesis that Otk and Drl might indeed interact in guiding axons along the anterior commissure. Furthermore, Wnt5 and Drl act antagonistically in two postembryonic brain centers, the mushroom body (MB) and the antennal lobes (AL) (MoreauFauvarque et al, 1998; Grillenzoni et al, 2007; Yao et al, 2007; Sakurai et al, 2009). In these systems, the Wnt5 signaling is apparently mediated by other receptors and based on its expression and mild mutant phenotypes, Otk and Otk2 are possible candidates. Supporting this hypothesis, it RNAi knockdown of otk was reported to result in loss or reduction of MB $\alpha / \beta$ lobes (Shimizu et al, 2011).

\subsubsection{Genetic interation of Otk/Otk2 with Fz}

Both Otk and Otk2 were shown to interact biochemically with Fz, even though the FzOtk interaction seemed to be rather weak (3.7, Fig. 42). Furthermore, otk,otk2;fz triple mutants were homozygous lethal when the zygotic or maternal components of all three genes were removed (3.7). However, this interaction and the resulting lethality were dependent on the nature of $f z$ alleles used (Table 18). The $f z^{22}$ mutant contains a mutation in the first intracellular loop and $f z^{P 21}$ harbors an insertion in the cysteine-rich domain. On the other hand, $f z^{R 52}$ and $f z^{H 51}$ contain a mutation in the third extracellular loop. Whereas $f z^{P 21}, f z^{R 52}$ and $f z^{H 511}$ are cell non-autonomous, $f z^{222}$ was shown to act cell-autonomously (Jones et al, 1996; Povelones et al, 2005).

Possibly, Otk acts as a receptor for Wnts in some pathways and as a co-receptor together with Frizzled proteins in others, resulting in the effects observed. Similarly, 
Ryk and Ror2 have been found to interact with Fz proteins even though they were shown to act as bona fide Wnt receptors in other systems (Lu et al, 2004a; Yamamoto et al, 2008). It is possible that these proteins function as co-receptors in a certain context. However, it is difficult to study, especially in vivo, whether proteins function as co-receptors in the same cell or as independent receptors on different cells. Likewise, Otk and Ror were found to interact biochemically as well (data not shown). Unfortunately, it is impossible to functionally classify these interactions as long as the main Otk/Otk2 ligands and potential signaling pathways have not been fully characterized.

\subsection{Possible molecular mechanisms of Wnt/Otk signaling}

The existence of a plethora of Wnt receptors suggests that they interact, leading to different signaling outcomes. Furthermore, it is not unlikely that many of them act redundantly. In the case of Drosophila Otk and 0tk2 this is most probably the case since even a double knock out of both genes did not lead to any clear phenotypes in any of the Wnt pathways investigated. Based on previous expression and functional analysis one might speculate on potential molecular mechanisms regarding Wnt/Otk signaling.

\subsubsection{Interaction with other receptors}

Akin to its vertebrate homolog PTK7, Otk/Otk2 might be involved in the fine-tuning of signaling outcome. One model for PTK7 function is that a ROR2/Fz/Wnt complex could trigger PCP signaling, while the Lrp/Fz/Wnt complex could activate canonical Wht signaling. Individual Wnts would favour the formation of one type of complex, thus explaining why some Wnts tend to activate PCP while others activate canonical signaling. In this context, PTK7, which binds the canonical Wnt3a and Wnt8 (Peradziryi et al, 2011), could inhibit canonical signaling by displacing LRP from the activating tripartite complex (Vincent \& Beckett, 2011). It is proposed that PTK7 traps canonical Wnts in a non-canonical receptor complex thereby preventing them from activating canonical Wnt signaling (Peradziryi et al, 2011, 2012).

Regarding Wnt5-Drl/Ryk interaction regulating axon guidance in Drosophila and vertebrates as well as Mom-2/Lin-18 interaction controlling C. elegans vulval 
development, the Ryk homologs are not considered to function as co-receptors but rather as bona fide Wnt receptors (Angers \& Moon, 2009). In contrast, it was demonstrated for HEK293 cells that Wnt, Fz and Ryk form a ternary complex required for the activation of a $\beta$-catenin-TCF luciferase reporter ( $\mathrm{Lu}$ et al, 2004a). Thus, Ryk seems to be both physically and functionally linked to canonical Wnt pathway components.

Similar models of function are also conceivable for Otk, however, it is urgently necessary to determine biochemically which Drosophila Whts are bound by Otk/Otk2. Furthermore, it will be interesting to investigate whether Otk and 0tk2 interact with other non-canonical receptors like Ror and Derailed and which signaling pathways might be influenced by these interactions. Unfortunately, no loss-of-function data are available for Drosophila Ror, making it impossible at the moment to perform genetic analysis which could determine whether Ror and Otk overlap in function or perform distinct roles.

In addition, it is likely that 0tk/0tk2 also bind to receptors that are not directly associated with Wnt signal transduction. In this context, PTK7 and Otk were reported to interact with Plexins (Winberg et al, 2001; Wagner et al, 2010). This is a family of transmembrane receptors which bind ligands of the Semaphorin class and regulate axon guidance and general cell-cell communication (Peradziryi et al, 2012). In Xenopus, PTK7 and PlexinA1 affect cranial neural crest cell migration. Similarly, in Drosophila, Otk and PlexinA1 were reported to mediate Semaphorin1A-induced repulsive axon guidance, yet these results were based on the $t^{3} k^{3}$ loss-of-function allele, which most likely carried a lethal second site hit, as discussed in 4.2. In light of a potential role of Otk/Otk2 in Wnt5/Drl-mediated repulsive axon guidance (see 4.4.2), it will be exciting to re-evaluate a possible connection between Otk/Otk2 and plexins.

\subsubsection{Downstream signaling}

The mechanism of vertebrate PTK7 as well as Drosophila Otk downstream signaling is still largely unknown. So far, no kinase activity could be reported for any of the homologs and, similarly to Derailed, they are believed to act as inactive kinases. The question arises, how apparently inactive Otk receptors would transduce Wnt signals. Different mechanisms are possible based on obervations in other systems. 
Inactive Otk receptors might recruit catalytically active downstream effectors. In this context it was shown that Xenopus PTK7 is required for Dsh localization to the plasma membrane in (Shnitsar \& Borchers, 2008) and this recruitment requires the adaptor proteins receptor of activated C kinase (RACK1) and protein kinase C $\delta 1$ (PKC $\delta 1$ ) (Wehner et al, 2011). It remains to be investigated whether Otk is also involved in Dsh membrane recruitment and which other components might be required to achieve this.

Similarly, Drl lacking its C-terminal domain fails to misguide axons to the anterior path, indicating that Drl might also engage a cytoplasmic signaling pathway (Bonkowsky et al, 1999). Indeed, $d r l$ and $\operatorname{src} 64 B$, a member of the Src family of tyrosine kinases, were found to interact during the formation of commissures during embryonic CNS developement (Wouda et al, 2008).

Another conceivable model of Otk action is protein cleavage and subsequent secretion or translocation of cleaved parts. Vertebrate PTK7 has recently been shown to be a target of membrane type-1 matrix metalloproteinase (MT1-MMP/MMP14). MT1-MMP recognizes a cleavage site in the seventh immunoglobulin domain of PTK7, thereby generating an extracellular fragment that is secreted and increases cell invasion. Since inhibition of MT1-MMP leads to similar convergent extension defects in zebrafish as does PTK7 loss of function, MT1-MMP-mediated proteolysis of PTK7 seems to be required for proper development (Golubkov et al, 2010; Peradziryi et al, 2012). Similarly, the introduction of an additional MT1-MMP cleavage site results in aberrant PTK7 proteolysis (Paudyal et al, 2010). These findings suggest that a tightly balanced ratio of membrane-bound and secreted fractions is indispensable for the proper function of vertebrate PTK7.

In case of Drl, it was shown that the Ryk sequence contains a $\gamma$-secretase cleavage site in its transmembrane region and that a C-terminal proteolytic fragment is released upon cleavage. Wnt3a promotes nuclear translocation of the Ryk intracellular domain, which is important for neural progenitor cell differentiation (Lyu et al, 2008). 


\section{SUMMARY AND CONCLUSIONS}

Canonical and non-canonical Wnt signaling pathways have in common Frizzled (Fz) as the core Wnt receptor element. However, in the past decade, other transmembrane receptors have been shown to bind Wnt ligands, resulting in the activation of diverse intracellular signaling pathways. Apart from members of the Frizzled receptor family, Wnt receptors include the low-density lipoprotein receptor-related protein (LRP, Drosophila Arrow), the protein tyrosine kinase 7 (PTK7) as well as the receptor tyrosine kinase-like orphan receptor (Ror) and the related to tyrosine kinase (Ryk) families. Wnt interactions with these receptors often lead to cellular effects that are unrelated to $\beta$-catenin, possibly mediating a variety of non-canonical signaling pathways.

Vertebrate PTK7 (protein tyrosine kinase 7) encodes a catalytically inactive receptortyrosine kinase and is required for the control of planar cell polarity (PCP) in frogs and mice by acting as Fz co-receptor and inhibiting canonical Wnt signaling. PTK7 therefore also seems to belong to this group of co-receptors required for pathway selection, however, the exact molecular mechanism remains as well as its conservation between different species remain to be clarified.

In this work I describe the characterization of the Drosophila PTK7 homolog Off-track (Otk) and its paralog Otk2. Otk and its paralog Otk2 are co-expressed throughout embryonic and larval development. They are highly expressed in the visceral mesoderm as well as in the nervous system and enriched at anterior commissures. Otk and Otk2 interact biochemically and possibly function redundantly in Wnt signal transduction. Mutation of otk was reported not to cause PCP phenotypes in the fly, but is suggested to block canonical Wingless signaling in embryonic patterning. I found that in contrast to previous reports, flies homozygous for a complete knock-out of otk are viable and fertile and indeed do not show PCP phenotypes. Surprisingly, flies homozygous for a double knock-out of otk and otk2 are viable as well and neither show PCP nor Wingless signaling phenotypes. However, otk,otk2 double mutants are male sterile due to an obstruction in the ejaculatory duct and this is possibly due disturbed Wnt2 signaling. Overall defects in the nervous system cannot be observed, most likely because only a subset of neurons is affected. This could be explained by the abundance of receptors and co-receptors acting in a redundant manner. Indeed, expression data suggest a possible connection to Wnt5/Drl signaling. Furthermore, 
genetic and biochemical studies revealed that otk,otk2 genetically and biochemically interact with $f z 1$, indicating that Otk/Otk2 might also function as Fz1 co-receptors in the signal transduction of Wingless or other members of the Wnt family under certain circumstances.

In summary, these results suggest that Otk and 0tk2 indeed function as redundant receptors in several Drosophila Wnt signaling pathways, including Wnt2 as well as Wnt5/Drl signaling. 


\section{BIBLIOGRAPHY}

Aberle H, Bauer A, Stappert J, Kispert A \& Kemler R (1997) Beta-Catenin Is a Target for the Ubiquitin-Proteasome Pathway. The EMBO Journal 16: 3797-804

Adler PN, Krasnow RE \& Liu J (1997) Tissue polarity points from cells that have higher Frizzled levels towards cells that have lower Frizzled levels. Current Biology 7: 940-9

Afzal AR, Rajab A, Fenske CD, Oldridge M, Elanko N, Ternes- E, Tüysüz B, Murday VA, Patton MA, Wilkie AOM \& Jeffery S (2000) Recessive Robinow syndrome, allelic to dominant brachydactyly type B , is caused by mutation of ROR2. Nature 25: 419-422

Van Amerongen R, Mikels A \& Nusse R (2008) Alternative Wnt signaling is initiated by distinct receptors. Science Signaling 1: re9

Van Amerongen R \& Nusse R (2009) Towards an integrated view of Wht signaling in development. Development 136: 3205-14

Amit S, Hatzubai A, Birman Y, Andersen JS, Ben-Shushan E, Mann M, Ben-Neriah Y \& Alkalay I (2002) Axin-mediated CKI phosphorylation of beta-catenin at Ser 45: a molecular switch for the Wnt pathway. Genes \& development 16: 1066-76

Angers S \& Moon RT (2009) Proximal events in Wnt signal transduction. Nature Reviews Molecular Cell Biology 10: 468-77

Axelrod JD \& McNeill H (2002) Coupling planar cell polarity signaling to morphogenesis. The Scientific World Journal 2: 434-54

Axelrod JD, Miller JR, Shulman JM, Moon RT \& Perrimon N (1998) Differential recruitment of Dishevelled provides signaling specificity in the planar cell polarity and Wingless signaling pathways. Genes \& Development 12: 2610-2622

Bachmann A \& Knust E (2008) The use of P-element transposons to generate transgenic flies. Methods in Molecular Biology 420: 61-77

Bakal C, Linding R, Llense F, Heffern E, Martin-Blanco E, Pawson T \& Perrimon N (2008) Phosphorylation networks regulating JNK activity in diverse genetic backgrounds. Science 322: 453-6

Baker NE (1987) Molecular cloning of sequences from wingless, a segment polarity gene in Drosophila: the spatial distribution of a transcript in embryos. The EMBO Journal 6: 1765-73

Barker N \& Clevers H (2006) Mining the Wnt pathway for cancer therapeutics. Nature Reviews Drug Discovery 5: 997-1014

Behrens J, Von Kries J, Kühl M, Bruhn L, Wedlich D, Grosschedl R \& Birchmeier W (1996) Functional interaction of beta-catenin with the transcription factor LEF-1. Nature 382: 638-642 
Bejsovec A \& Martinez Arias A (1991) Roles of wingless in patterning the larval epidermis of Drosophila. Development 113: 471-85

Bejsovec A \& Wieschaus E (1993) Segment polarity gene interactions modulate epidermal patterning in Drosophila embryos. Development 119: 501-17

Bhanot P, Brink M, Harryman Samos C, Hsieh J, Wang Y, Macke J, Andrew D, Nathans J \& Nusse R (1996) A new member of the frizzled family from Drosophila functions as a Wingless receptor. Nature 382: 225-230

Bhanot P, Fish M, Jemison J a, Nusse R, Nathans J \& Cadigan KM (1999) Frizzled and Dfrizzled2 function as redundant receptors for Wingless during Drosophila embryonic development. Development 126: 4175-86

Bhat KM (1998) Frizzled and Frizzled 2 Play a Partially Redundant Role in Wingless Signaling and Have Similar Requirements To Wingless in Neurogenesis. Cell 95: 1027-36

Bienz M (1994) Homeotic genes and positional signalling in the Drosophila viscera. Trends in Genetics 10: 22-6

Billiard J, Way DS, Seestaller-wehr LM, Moran RA, Mangine A \& Bodine PVN (2005) The Orphan Receptor Tyrosine Kinase Ror2 Modulates Canonical Wnt Signaling in. Molecular Endocrinology 19: 90-101

Bischof J, Maeda RK, Hediger M, Karch F \& Basler K (2007) An optimized transgenesis system for Drosophila using germ-line-specific phiC31 integrases. Proceedings of the National Academy of Sciences 104: 3312-7

Bokhoven H Van, Celli J, Kayserili H, Beusekom E Van, Balci S, Brussel W, Skovby F, Kerr B, Percin EF, Akarsu N \& Brunner HG (2000) Mutation of the gene encoding the ROR2 tyrosine kinase causes autosomal recessive Robinow syndrome. Nature 25: 423-426

Bonkowsky JL, Yoshikawa S, O'Keefe DD, Scully AL \& Thomas JB (1999) Axon routing across the midline controlled by the Drosophila Derailed receptor. Nature 402: 2-6

Boutros M, Paricio N, Strutt DI \& Mlodzik M (1998) Dishevelled activates JNK and discriminates between JNK pathways in planar polarity and wingless signaling. Cell 94: 109-18

Brand AH \& Perrimon N (1993) Targeted gene expression as a means of altering cell fates and generating dominant phenotypes. Development 415: 401-415

Buratovich MA, Anderson S, Gieseler K, Pradel J \& Wilder EL (2000) DWnt-4 and Wingless have distinct activities in the Drosophila dorsal epidermis. Dev Genes Evol 210: 111-119

Cabrera C V, Alonso MC, Johnston P, Phillips RG \& Lawrence PA (1987) Phenocopies induced with antisense RNA identify the wingless gene. Cell 50: 659-63

Cafferty P, Yu L \& Rao Y (2004) The receptor tyrosine kinase Off-track is required for layerspecific neuronal connectivity in Drosophila. Development 131: 5287-95

Callahan C, Muralidhar M, Lundgren S, Scully A \& Thomas J (1995) Control of neuronal pathway selection by a Drosophila receptor protein-tyrosine kinase family member. Nature 376: 171-4 
Callahan CA, Bonkovsky JL, Scully AL \& Thomas JB (1996) Derailed is required for muscle attachment site selection in Drosophila. Development 122: 171-4

Cao J, Li Y, Xia W, Reddig K, Hu W, Xie W, Li H-S \& Han J (2011) A Drosophila metallophosphoesterase mediates deglycosylation of rhodopsin. The EMBO Journal 30: 3701-13

Cavallo R, Cox R, Moline M, Roose J, Polevoy GA, Clevers H, Peifer M \& Bejsovec A (1998) Drosophila Tcf and Groucho interact to repress Wingless signalling activity. Nature 395: 604-608

Cha S-W, Tadjuidje E, Tao Q, Wylie C \& Heasman J (2008) Wnt5a and Wnt11 interact in a maternal Dkk1-regulated fashion to activate both canonical and non-canonical signaling in Xenopus axis formation. Development 135: 3719-29

Chen CM \& Struhl G (1999) Wingless transduction by the Frizzled and Frizzled2 proteins of Drosophila. Development 126: 5441-52

Chou YH \& Hayman MJ (1991) Characterization of a member of the immunoglobulin gene superfamily that possibly represents an additional class of growth factor receptor. Proceedings of the National Academy of Sciences 88: 4897-901

Chu-LaGraff Q \& Doe CQ (1993) Neuroblast specification and formation regulated by wingless in the Drosophila CNS. Science 261: 1594-7

Clevers H (2006) Wnt/beta-catenin signaling in development and disease. Cell 127: 469-80

Cohen ED, Mariol M-C, Wallace RMH, Weyers J, Kamberov YG, Pradel J \& Wilder EL (2002) DWnt4 regulates cell movement and focal adhesion kinase during Drosophila ovarian morphogenesis. Developmental Cell 2: 437-48

Couso JP, Bishop S a \& Martinez Arias A (1994) The wingless signalling pathway and the patterning of the wing margin in Drosophila. Development 120: 621-36

De Cuevas M \& Matunis EL (2011) The stem cell niche: lessons from the Drosophila testis. Development 138: 2861-9

Daniels DL \& Weis WI (2005) Beta-catenin directly displaces Groucho/TLE repressors from Tcf/Lef in Wnt-mediated transcription activation. Nature Structural \& Molecular Biology 12: $364-71$

Davidson G, Wu W, Shen J, Bilic J, Fenger U, Stannek P, Glinka A \& Niehrs C (2005) Casein kinase 1 gamma couples Wnt receptor activation to cytoplasmic signal transduction. Nature 438: 867-72

DeFalco T, Camara N, Le Bras S \& Van Doren M (2008a) Nonautonomous sex determination controls sexually dimorphic development of the Drosophila gonad. Developmental Cell 14: $275-86$

DeFalco T, Camara N, Le Bras S \& Van Doren M (2008b) Nonautonomous sex determination controls sexually dimorphic development of the Drosophila gonad. Developmental Cell 14: $275-86$ 
DiNardo S, Kuner JM, Theis J \& O'Farrell PH (1985) Development of embryonic pattern in D. melanogaster as revealed by accumulation of the nuclear engrailed protein. Cell 43: 5969

Dominguez I, Itoh K \& Sokol SY (1995) Role of glycogen synthase kinase 3 beta as a negative regulator of dorsoventral axis formation in Xenopus embryos. Proceedings of the National Academy of Sciences 92: 8498-502

Dougan S \& DiNardo S (1992) Drosophila wingless generates cell type diverstiy among engrailed expressing cells. Nature 360: 347-350

Du SJ, Purcell SM, Christian JL, McGrew L \& Moon RT (1995) Identification of Distinct Classes and Functional Domains of Wnts through Expression of Wild-type and Chimeric Proteins in Xenopus Embryos . Molecular and Cellular Biology 15: 2625-2634

Dura J, Preat T \& Tully TIM (1993) Identification of linotte, a new gene affecting learning and memory in Drosophila melanogaster. J. Neurogenetics 9: 1-14

Dura JM, Taillebourg E \& Préat T (1995) The Drosophila learning and memory gene linotte encodes a putative receptor tyrosine kinase homologous to the human RYK gene product. FEBS letters 370: $250-4$

Eisenberg L, Ingham P \& Brown A (1992) Cloning and Characterization of a Novel Drosophila Wnt Gene, Dwnt-5, A Putative Downstream Target of the Homeobox Gene Distal-less. Developmental Biology 83: 73-83

Estrada B, Casares F \& Sánchez-Herrero E (2003) Development of the genitalia in Drosophila melanogaster. Differentiation 71: 299-310

Fish MP, Groth AC, Calos MP \& Nusse R (2007) Creating transgenic Drosophila by microinjecting the site-specific phiC31 integrase mRNA and a transgene-containing donor plasmid. Nature Protocols 2: 2325-31

Forrester WC (2002) The Ror receptor tyrosine kinase family. Cell Mol Life Sci 59: 83-96

Forrester WC, Dell M, Perens E \& Garriga G (1999) A C . elegans Ror receptor tyrosine kinase regulates cell motility and asymmetric cell division. Nature 400: 717-721

Forrester WC, Kim C \& Garriga G (2004) The Caenorhabditis elegans Ror RTK CAM-1 inhibits EGL-20/Wnt signaling in cell migration. Genetics 168: 1951-62

Fradkin LG, Dura J-M \& Noordermeer JN (2010) Ryks: new partners for Wnts in the developing and regenerating nervous system. Trends in Neurosciences 33: 84-92

Fradkin LG, Noordermeer JN \& Nusse R (1995) The Drosophila Wnt protein DWnt-3 is a secreted glycoprotein localized on the axon tracts of the embryonic CNS. Developmental Biology 168: 202-213

Fradkin LG, Van Schie M, Wouda RR, De Jong A, Kamphorst JT, Radjkoemar-Bansraj M \& Noordermeer JN (2004) The Drosophila Wnt5 protein mediates selective axon fasciculation in the embryonic central nervous system. Developmental Biology 272: 36275 
Ganguly A, Jiang J \& Ip YT (2005) Drosophila WntD is a target and an inhibitor of the Dorsal/Twist/Snail network in the gastrulating embryo. Development 132: 3419-29

Gao C \& Chen Y (2010) Dishevelled : The hub of Wnt signaling. Cell Signal 22: 717-27

Gieseler K, Graba Y, Mariol MC, Wilder EL, Martinez-Arias A, Lemaire P \& Pradel J (1999) Antagonist activity of DWnt-4 and wingless in the Drosophila embryonic ventral ectoderm and in heterologous Xenopus assays. Mechanisms of Development 85: 123-31

Gieseler K, Mariol MC, Sagnier T, Graba Y \& Pradel J (1995) Wingless and DWnt4, 2 Drosophila Wnt genes, have related expression, regulation and function during the embryonic development. Comptes rendus de l'Académie des sciences. Série III, Sciences de la vie 318: 1101-10

Gieseler K, Wilder E, Mariol MC, Buratovitch M, Bérenger H, Graba Y \& Pradel J (2001) DWnt4 and wingless elicit similar cellular responses during imaginal development. Developmental Biology 232: 339-50

Golic KG \& Lindquist S (1989) The FLP recombinase of yeast catalyzes site-specific recombination in the Drosophila genome. Cell 59: 499-509

Golubkov VS, Chekanov A V, Cieplak P, Aleshin AE, Chernov A V, Zhu W, Radichev IA, Zhang D, Dong PD \& Strongin AY (2010) The Wnt/planar cell polarity protein-tyrosine kinase-7 (PTK7) is a highly efficient proteolytic target of membrane type-1 matrix metalloproteinase: implications in cancer and embryogenesis. The Journal of Biological Chemistry 285: 35740-9

Gordon MD, Ayres JS, Schneider DS \& Nusse R (2008) Pathogenesis of listeria-infected Drosophila wntD mutants is associated with elevated levels of the novel immunity gene edin. PLoS Pathogens 4: e1000111

Gordon MD, Dionne MS, Schneider DS \& Nusse R (2005) WntD is a feedback inhibitor of Dorsal/NF-kappaB in Drosophila development and immunity. Nature 437: 746-9

Gordon MD \& Nusse R (2006) Wnt signaling: multiple pathways, multiple receptors, and multiple transcription factors. The Journal of Biological Chemistry 281: 22429-33

Graba Y, Gieseler K, Aragnol D, Laurenti P, Mariol MC, Berenger H, Sagnier T \& Pradel J (1995) DWnt-4, a novel Drosophila Wnt gene acts downstream of homeotic complex genes in the visceral mesoderm. Development 121: 209-18

Green JL, Inoue T \& Sternberg PW (2007) The C. elegans ROR receptor tyrosine kinase, CAM1, non-autonomously inhibits the Wnt pathway. Development 134: 4053-62

Green JL, Kuntz SG \& Sternberg PW (2008) Ror receptor tyrosine kinases: orphans no more. Trends in Cell Biology 18: 536-44

Grillenzoni N, Flandre A, Lasbleiz C \& Dura J (2007) Respective roles of the DRL receptor and its ligand WNT5 in Drosophila mushroom body development. Development 3097: 30893097

Groth AC, Fish M, Nusse R \& Calos MP (2004) Construction of Transgenic Drosophila by Using the Site-Specific Integrase. Genetics 1782: 1775-1782 
Grumolato L, Liu G, Mong P, Mudbhary R, Biswas R, Arroyave R, Vijayakumar S, Economides AN \& Aaronson SA (2010) Canonical and noncanonical Whts use a common mechanism to activate completely unrelated coreceptors. Genes \& Development 24: 2517-30

Guger KA \& Gumbiner BM (1995) Beta-Catenin has Wnt-like activity and mimics the Nieuwkoop signaling center in Xenopus dorsal-ventral patterning. Developmental Biology 172: 115-25

Gönczy P \& DiNardo S (1996) The germ line regulates somatic cyst cell proliferation and fate during Drosophila spermatogenesis. Development 122: 2437-47

He X, Saint-Jeannet J, Woodgett J, Varmus HE \& Dawid I (1995) Glycogen synthase kinase-3 and dorsoventral patterning in Xenopus embryos. Nature 374: 617-622

He X, Saint-Jeannet JP, Wang Y, Nathans J, Dawid I \& Varmus H (1997) A Member of the Frizzled Protein Family Mediating Axis Induction by Wnt-5A. Science 275: 1652-1654

Heisenberg CP, Tada M, Rauch G, Saúde L, Concha M, Geisler R, Stemple D, Smith J \& Wilson S (2000) Silberblick / Wnt11 mediates convergent extension movements during zebrafish gastrulation. Nature 405: 76-81

Van den Heuvel M, Nusse R, Johnston P \& Lawrence PA (1989) Distribution of the wingless gene product in Drosophila embryos: a protein involved in cell-cell communication. Cell 59: $739-49$

Hikasa H, Shibata M, Hiratani I \& Taira M (2002) The Xenopus receptor tyrosine kinase Xror2 modulates morphogenetic movements of the axial mesoderm and neuroectoderm via Wnt signaling. Development 129: 5227-39

Ho H-YH, Susman MW, Bikoff JB, Ryu YK, Jonas AM, Hu L, Kuruvilla R \& Greenberg ME (2012) Wnt5a-Ror-Dishevelled signaling constitutes a core developmental pathway that controls tissue morphogenesis. Proceedings of the National Academy of Sciences 109: 4044-51

Hoffmans R, Städeli R \& Basler K (2005) Pygopus and legless provide essential transcriptional coactivator functions to armadillo/beta-catenin. Current Biology 15: 1207-11

Hovens CM, Stacker SA, Andres AC, Harpur AG, Ziemiecki A \& Wilks AF (1992) RYK, a receptor tyrosine kinase-related molecule with unusual kinase domain motifs. Proceedings of the National Academy of Sciences 89: 11818-22

Immerglück K, Lawrence PA \& Bienz M (1990) Induction Mediated across Germ Layers in Drosophila by a Genetic Cascade. Cell 62: 261-266

Ingham P (1988) The molecular genetics of embryonic pattern formation in Drosophila. Nature 335: 25-34

Inoue T, Oz HS, Wiland D, Gharib S, Deshpande R, Hill RJ, Katz WS, Sternberg PW, Street R \& Avenue W (2004) C . elegans LIN-18 Is a Ryk Ortholog and Functions in Parallel to LIN17 / Frizzled in Wnt Signaling. Cell 118: 795-806

Jakobsen JS, Braun M, Astorga J, Gustafson EH, Sandmann T, Karzynski M, Carlsson P \& Furlong EEM (2007) Temporal ChIP-on-chip reveals Biniou as a universal regulator of the visceral muscle transcriptional network. Genes \& Development 21: 2448-60 
Janson K, Cohen ED \& Wilder EL (2001) Expression of DWnt6, DWnt10, and DFz4 during Drosophila development. Mechanisms of Development 103: 117-20

Jones KH, Liu J \& Adler PN (1996) Molecular Analysis of EMS-Induced frizzled Mutations in Drosophila melanogaster. Genetics 142: 205-215

Jung J-W, Shin W-S, Song J \& Lee S-T (2004) Cloning and characterization of the full-length mouse Ptk7 cDNA encoding a defective receptor protein tyrosine kinase. Gene 328: 7584

Keeble TR, Halford MM, Seaman C, Kee N, Macheda M, Anderson RB, Stacker S a \& Cooper HM (2006) The Wnt receptor Ryk is required for Wnt5a-mediated axon guidance on the contralateral side of the corpus callosum. The Journal of Neuroscience 26: 5840-8

Kilian B, Mansukoski H, Barbosa FC, Ulrich F, Tada M \& Heisenberg C-P (2003) The role of $\mathrm{Ppt} / \mathrm{Wnt5}$ in regulating cell shape and movement during zebrafish gastrulation. Mechanisms of Development 120: 467-476

Kim C \& Forrester WC (2003) Functional analysis of the domains of the C. elegans Ror receptor tyrosine kinase CAM-1. Developmental Biology 264: 376-390

Kim G-H, Her J-H \& Han J-K (2008) Ryk cooperates with Frizzled 7 to promote Wnt11mediated endocytosis and is essential for Xenopus laevis convergent extension movements. The Journal of Cell Biology 182: 1073-82

Klingensmith J \& Nusse R (1994) Signaling by wingless in Drosophila. Developmental Biology 166: $396-414$

Kozopas KM \& Nusse R (2002) Direct Flight Muscles in Drosophila Develop from Cells with Characteristics of Founders and Depend on DWnt-2 for Their Correct Patterning. Developmental Biology 325: 312-325

Kozopas KM, Samos CH \& Nusse R (1998) DWnt-2, a Drosophila Wnt gene required for the development of the male reproductive tract, specifies a sexually dimorphic cell fate. Genes \& Development 12: 1155-65

Kramps T, Peter O, Brunner E, Nellen D, Froesch B, Chatterjee S, Murone M, Züllig S \& Basler K (2002) Wnt/wingless signaling requires BCL9/legless-mediated recruitment of pygopus to the nuclear beta-catenin-TCF complex. Cell 109: 47-60

Krasnow RE \& Adler PN (1994) A single frizzled protein has a dual function in tissue polarity. Development 120: 1883-93

Kucherenko MM, Marrone AK, Rishko VM, Magliarelli HDF \& Shcherbata HR (2011) Stress and muscular dystrophy: a genetic screen for dystroglycan and dystrophin interactors in Drosophila identifies cellular stress response components. Developmental Biology 352: 228-42

Kucherenko MM, Pantoja M, Yatsenko AS, Shcherbata HR, Fischer K a, Maksymiv D V, Chernyk YI \& Ruohola-Baker H (2008) Genetic modifier screens reveal new components that interact with the Drosophila dystroglycan-dystrophin complex. PloS One 3: e2418

Lasko PF \& Ashburner M (1990) Posterior localization of vasa protein correlates with, but is not sufficient for, pole cell development. Genes \& Development 4: 905-921 
Lemmon MA \& Schlessinger J (2010) Cell signaling by receptor tyrosine kinases. Cell 141: 1117-34

Li L, Hutchins BI \& Kalil K (2009) Wnt5a induces simultaneous cortical axon outgrowth and repulsive axon guidance through distinct signaling mechanisms. The Journal of Neuroscience 29: 5873-83

Liu C, Li Y, Semenov M, Han C, Baeg GH, Tan Y, Zhang Z, Lin X \& He X (2002) Control of betacatenin phosphorylation/degradation by a dual-kinase mechanism. Cell 108: 837-47

Liu Y, Shi J, Lu C-C, Wang Z-B, Lyuksyutova AI, Song X-J, Song X \& Zou Y (2005) Ryk-mediated Wnt repulsion regulates posterior-directed growth of corticospinal tract. Nature Neuroscience 8: 1151-9

Liu Y, Wang X, Lu C-C, Kerman R, Steward O, Xu X-M \& Zou Y (2008) Repulsive Wnt signaling inhibits axon regeneration after CNS injury. The Journal of neuroscience : the official journal of the Society for Neuroscience 28: 8376-82

Llimargas M \& Lawrence P a (2001) Seven Wnt homologues in Drosophila: a case study of the developing tracheae. Proceedings of the National Academy of Sciences 98: 14487-92

Logan CY \& Nusse R (2004) The Wnt signaling pathway in development and disease. Annual Review of Cell and Developmental Biology 20: 781-810

Lu W, Yamamoto V, Ortega B \& Baltimore D (2004a) Mammalian Ryk is a Wnt coreceptor required for stimulation of neurite outgrowth. Cell 119: 97-108

Lu X, Borchers AGM, Jolicoeur C, Rayburn H, Baker JC \& Tessier-lavigne M (2004b) PTK7 / CCK-4 is a novel regulator of planar cell polarity in vertebrates. Nature 430: 93-98

Lyu J, Yamamoto V \& Lu W (2008) Cleavage of the Wnt receptor Ryk regulates neuronal differentiation during cortical neurogenesis. Developmental Cell 15: 773-80

Marrone AK, Kucherenko MM, Rishko VM \& Shcherbata HR (2011) New dystrophin/dystroglycan interactors control neuron behavior in Drosophila eye. BMC Neuroscience 12: 93

McMahon a P \& Moon RT (1989) Ectopic expression of the proto-oncogene int-1 in Xenopus embryos leads to duplication of the embryonic axis. Cell 58: 1075-84

Mikels AJ \& Nusse R (2006) Purified Wnt5a protein activates or inhibits beta-catenin-TCF signaling depending on receptor context. PLoS Biology 4: e115

Miller MA \& Steele RE (2000) Lemon encodes an unusual receptor protein-tyrosine kinase expressed during gametogenesis in Hydra. Developmental Biology 224: 286-98

Minami Y, Oishi I, Endo M \& Nishita M (2010) Ror-family receptor tyrosine kinases in noncanonical Wnt signaling: their implications in developmental morphogenesis and human diseases. Developmental Dynamics 239: 1-15

Miyashita T, Koda M, Kitajo K, Yamazaki M, Takahashi K, Kikuchi A \& Yamashita T (2009) Wnt-Ryk signaling mediates axon growth inhibition and limits functional recovery after spinal cord injury. Journal of Neurotrauma 26: 955-64 
Molenaar M, Van de Wetering M, Oosterwegel M, Peterson-Maduro J, Godsave S, Korinek V, Roose J, Destrée O \& Clevers H (1996) XTcf-3 transcription factor mediates beta-catenininduced axis formation in Xenopus embryos. Cell 86: 391-9

Montcouquiol M, Crenshaw EB \& Kelley MW (2006) Noncanonical Wnt signaling and neural polarity. Annual Review of Neuroscience 29: 363-86

Moreau-Fauvarque C, Taillebourg E, Boissoneau E, Mesnard J \& Dura JM (1998) The receptor tyrosine kinase gene linotte is required for neuronal pathway selection in the Drosophila mushroom bodies. Mechanisms of Development 78: 47-61

Mossie K, Jallal B, Alves F, Sures I, Plowman GD \& Ullrich A (1995) Colon carcinoma kinase-4 defines a new subclass of the receptor tyrosine kinase family. Oncogene 11: 2179-2184

Müller H a, Samanta R \& Wieschaus E (1999) Wingless signaling in the Drosophila embryo: zygotic requirements and the role of the frizzled genes. Development 126: 577-86

Müller HAJ (2008) Immunolabelling of embryos. Methods in Molecular Biology 420: 1-11

Nanda S, DeFalco TJ, Loh SHY, Phochanukul N, Camara N, Van Doren M \& Russell S (2009) Sox100B, a Drosophila group E Sox-domain gene, is required for somatic testis differentiation. Sexual Development 3: 26-37

Noordermeer JN, Klingensmith J, Perrimon N \& Nusse R (1994) Dishevelled and armadillo act in the Wingless signalling pathway in Drosophila. Nature 367: 80-3

Nusse R \& Varmus HE (1982) Many tumors induced by the mouse mammary tumor virus contain a provirus integrated in the same region of the host genome. Cell 31: 99-109

Nüsslein-Volhard C \& Wieschaus E (1980) Mutations affecting segment number and polarity in Drosophila. Nature 287: 795-801

Oishi I, Sugiyama S, Liu Z \& Yamamura H (1997) A Novel Drosophila Receptor Tyrosine Kinase Expressed Specifically in the Nervous System. The Journal of Biological Chemistry 272: 11916-11923

Oishi I, Suzuki H, Onishi N, Takada R, Kani S, Ohkawara B, Koshida I, Suzuki K, Yamada G, Schwabe GC, Mundlos S, Shibuya H, Takada S \& Minami Y (2003) The receptor tyrosine kinase Ror2 is involved in non-canonical Wnt5a/JNK signalling pathway. Genes to Cells 8: $645-54$

Oldridge M, Fortuna AM, Maringa M, Propping P, Mansour S, Pollitt C, Dechiara TM, Kimble RB, Valenzuela DM, Yancopoulos GD \& Wilkie AOM (2000) Dominant mutations in ROR2 , encoding an orphan receptor tyrosine kinase, cause brachydactyly type B. Nature 24: 275-278

Olson J \& Papkoff J (1994) Regulated Expression of Wnt Family Members during Proliferation of C57mg Mammary Cells. Cell Growth \& Differentiation 5: 197-206

Parks AL, Cook KR, Belvin M, Dompe N a, Fawcett R, Huppert K, Tan LR, Winter CG, Bogart KP, Deal JE, Deal-Herr ME, Grant D, Marcinko M, Miyazaki WY, Robertson S, Shaw KJ, Tabios M, Vysotskaia V, Zhao L, Andrade RS, et al (2004) Systematic generation of highresolution deletion coverage of the Drosophila melanogaster genome. Nature Genetics 36: $288-92$ 
Patel NH, Schafer B, Goodman CS \& Holmgren R (1989) The role of segment polarity genes during Drosophila neurogenesis. Genes \& Development 3: 890-904

Patthy L (2000) The WIF module. Trends Biochem Sci 25: 12-13

Paudyal A, Damrau C, Patterson VL, Ermakov A, Formstone C, Lalanne Z, Wells S, Lu X, Norris DP, Dean CH, Henderson DJ \& Murdoch JN (2010) The novel mouse mutant, chuzhoi, has disruption of Ptk7 protein and exhibits defects in neural tube, heart and lung development and abnormal planar cell polarity in the ear. BMC Developmental Biology 10: 87

Peradziryi H, Kaplan N a, Podleschny M, Liu X, Wehner P, Borchers A \& Tolwinski NS (2011) PTK7/Otk interacts with Wnts and inhibits canonical Wnt signalling. The EMBO Journal 30: $3729-40$

Peradziryi H, Tolwinski NS \& Borchers A (2012) The many roles of PTK7: A versatile regulator of cell-cell communication. Archives of Biochemistry and Biophysics 524: 71-6

Perrimon N \& Mahowald a P (1987) Multiple functions of segment polarity genes in Drosophila. Developmental biology 119: 587-600

Pinson KI, Brennan J, Monkley S, Avery BJ \& Skarnes WC (2000) An LDL-receptor-related protein mediates Wnt signalling in mice. Nature 407: 535-8

Povelones M, Howes R, Fish M \& Nusse R (2005) Genetic evidence that Drosophila frizzled controls planar cell polarity and Armadillo signaling by a common mechanism. Genetics 171: $1643-54$

Pulido D, Campuzano S, Koda T, Modolell J \& Barbacid M (1992) Dtrk, a Drosophila gene related to the trk family of neurotrophin receptors, encodes a novel class of neural cell adhesion molecule. The EMBO Journal 11: 391-404

Puppo F, Thomé V, Lhoumeau A-C, Cibois M, Gangar A, Lembo F, Belotti E, Marchetto S, Lécine P, Prébet T, Sebbagh M, Shin W-S, Lee S-T, Kodjabachian L \& Borg J-P (2011) Protein tyrosine kinase 7 has a conserved role in $\mathrm{Wnt} / \beta$-catenin canonical signalling. EMBO Reports 12: 43-9

Qian D, Jones C, Rzadzinska A, Mark S, Zhang X, Steel KP, Dai X \& Chen P (2007) Wnt5a functions in planar cell polarity regulation in mice. Developmental Biology 306: 121-33

Raja SJ \& Renkawitz-Pohl R (2005) Replacement by Drosophila melanogaster Protamines and Mst77F of Histones during Chromatin Condensation in Late Spermatids and Role of Sesame in the Removal of These Proteins from the Male Pronucleus. Molecular and Cellular Biology 25: 6165-6177

Reya T \& Clevers H (2005) Wnt signalling in stem cells and cancer. Nature 434: 843-50

Rijsewijk F, Schuermann M, Wagenaar E, Parren P, Weigel D \& Nusse R (1987) The Drosophila homolog of the mouse mammary oncogene int- 1 is identical to the segment polarity gene wingless. Cell 50: 649-57

Roose J, Molenaar M, Peterson J, Hurenkamp J, Brantjes H, Moerer P, Van de Wetering M, Destrée 0 \& Clevers H (1998) The Xenopus Wnt effector XTcf-3 interacts with Grouchorelated transcriptional repressors. Nature 395: 608-12 
Rubin GM \& Spradling a C (1982) Genetic transformation of Drosophila with transposable element vectors. Science 218: 348-53

Rulifson EJ, Wu C \& Nusse R (2000) by the Bifunctional Receptor Frizzled Is Determined by Affinity for Wingless. Molecular Cell 6: 117-126

Russell J, Gennissen A \& Nusse R (1992) Isolation and expression of two novel Wnt/wingless gene homologues in Drosophila. Development 115: 475-85

Sakurai M, Aoki T, Yoshikawa S, Santschi L a, Saito H, Endo K, Ishikawa K, Kimura K, Ito K, Thomas JB \& Hama C (2009) Differentially expressed Drl and Drl-2 play opposing roles in Wnt5 signaling during Drosophila olfactory system development. The Journal of Neuroscience 29: 4972-80

Sato M, Umetsu D, Murakami S, Yasugi T \& Tabata T (2006) DWnt4 regulates the dorsoventral specificity of retinal projections in the Drosophila melanogaster visual system. Nature Neuroscience 9: 67-75

Savory JGA, Mansfield M, Rijli FM \& Lohnes D (2011) Cdx mediates neural tube closure through transcriptional regulation of the planar cell polarity gene Ptk7. Development 138: $1361-70$

Schambony A \& Wedlich D (2007) Wnt-5A/Ror2 regulate expression of XPAPC through an alternative noncanonical signaling pathway. Developmental Cell 12: 779-92

Schmidt-Ott U \& Technau GM (1992) Expression of en and wg in the embryonic head and brain of Drosophila indicates a refolded band of seven segment remnants. Development 116: $111-25$

Schmitt AM, Shi J, Wolf AM, Lu C-C, King L a \& Zou Y (2006) Wnt-Ryk signalling mediates medial-lateral retinotectal topographic mapping. Nature 439: 31-7

Schneider I (1972) Cell lines derived from late embryonic stages of Drosophila melanogaster. Journal of Embryology and Experimental Morphology 27: 353-65

Schwabe GC, Tinschert S, Buschow C, Meinecke P, Wolff G, Gillessen-kaesbach G, Oldridge M, Wilkie AOM, Ko R \& Mundlos S (2000) Distinct Mutations in the Receptor Tyrosine Kinase Gene ROR2 Cause Brachydactyly Type B. Am J Hum Genet: 822-831

Seifert JRK \& Mlodzik M (2007) Frizzled/PCP signalling: a conserved mechanism regulating cell polarity and directed motility. Nature Reviews Genetics 8: 126-38

Sharma R \& Chopra VL (1976) Effect of the Wingless Development (wg1) Mutation on Wing and Haltere in Drosophila melanogaster. Developmental Biology 465: 461-465

Shimizu H, Julius M, Giarré M, Zheng Z, Brown A \& Kitajewski J (1997) Transformation by Wnt Family Proteins Correlates with Regulation of beta-catenin. Cell Growth \& Differentiation 8: 1349-1358

Shimizu K, Sato M \& Tabata T (2011) The Wnt5/planar cell polarity pathway regulates axonal development of the Drosophila mushroom body neuron. The Journal of Neuroscience 31: 4944-54 
Shnitsar I \& Borchers A (2008) PTK7 recruits dsh to regulate neural crest migration. Development 4024: 4015-4024

Siegfried E, Chou TB \& Perrimon N (1992) Wingless signaling acts through zeste-white 3, the Drosophila homolog of glycogen synthase kinase-3, to regulate engrailed and establish cell fate. Cell 71: 1167-79

Simon a F, Boquet I, Synguélakis M \& Préat T (1998) The Drosophila putative kinase linotte (derailed) prevents central brain axons from converging on a newly described interhemispheric ring. Mechanisms of Development 76: 45-55

Skaer H \& Martinez Arias A (1992) The wingless product is required for cell proliferation in the Malpighian tubule anlage of Drosophila melanogaster. Development 754: 745-754

Stacker SA, Hovens CM, Vitali A, Pritchard MA, Baker E, Sutherland GR \& Wilks a F (1993) Molecular cloning and chromosomal localisation of the human homologue of a receptor related to tyrosine kinases (RYK). Oncogene 8: 1347-56

Sternberg PW \& Horvitz HR (1988) lin-17 mutations of Caenorhabditis elegans disrupt certain asymmetric cell divisions. Developmental Biology 130: 67-73

Städeli R \& Basler K (2005) Dissecting nuclear Wingless signalling: recruitment of the transcriptional co-activator Pygopus by a chain of adaptor proteins. Mechanisms of Development 122: 1171-82

Susic-Jung L, Hornbruch-Freitag C, Kuckwa J, Rexer K-H, Lammel U \& Renkawitz-Pohl R (2012) Multinucleated smooth muscles and mononucleated as well as multinucleated striated muscles develop during establishment of the male reproductive organs of Drosophila melanogaster. Developmental Biology 370: 86-97

Tamai K, Semenov M, Kato Y, Spokony R, Liu C, Katsuyama Y, Hess F, Saint-Jeannet JP \& He X (2000) LDL-receptor-related proteins in Wnt signal transduction. Nature 407: 530-5

Tamura K, Peterson D, Peterson N, Stecher G, Nei M \& Kumar S (2011) MEGA5: molecular evolutionary genetics analysis using maximum likelihood, evolutionary distance, and maximum parsimony methods. Molecular Biology and Evolution 28: 2731-9

Tao Q, Yokota C, Puck H, Kofron M, Birsoy B, Yan D, Asashima M, Wylie CC, Lin X \& Heasman J (2005) Maternal wnt11 activates the canonical wnt signaling pathway required for axis formation in Xenopus embryos. Cell 120: 857-71

Thibault ST, Singer M a, Miyazaki WY, Milash B, Dompe N a, Singh CM, Buchholz R, Demsky M, Fawcett R, Francis-Lang HL, Ryner L, Cheung LM, Chong A, Erickson C, Fisher WW, Greer K, Hartouni SR, Howie E, Jakkula L, Joo D, et al (2004) A complementary transposon tool kit for Drosophila melanogaster using P and piggyBac. Nature Genetics 36: 283-7

Thüringer F \& Bienz M (1993) Indirect autoregulation of a homeotic Drosophila gene mediated by extracellular signaling. Proceedings of the National Academy of Sciences 90: 3899-903

Veeman MT, Axelrod JD \& Moon RT (2003) A second canon. Functions and mechanisms of beta-catenin-independent Wnt signaling. Developmental Cell 5: 367-77 
Vincent J-P \& Beckett K (2011) Off-track takes Frizzled off the canonical path. The EMBO Journal 30: 3665-6

Wagner G, Peradziryi H, Wehner P \& Borchers A (2010) PlexinA1 interacts with PTK7 and is required for neural crest migration. Biochemical and Biophysical Research Communications 402: 402-7

Wallingford JB \& Habas R (2005) The developmental biology of Dishevelled: an enigmatic protein governing cell fate and cell polarity. Development 132: 4421-36

Wallingford JB, Vogeli KM \& Harland RM (2001) Regulation of convergent extension in Xenopus by Wnt5a and Frizzled-8 is independent of the canonical Wnt pathway. The International Journal of Developmental Biology 45: 225-7

Wang MC, Bohmann D \& Jasper H (2003) JNK signaling confers tolerance to oxidative stress and extends lifespan in Drosophila. Developmental Cell 5: 811-6

Weber K \& Osborn M (1969) The Reliability of Molecular Weight Determinations by Dodecyl Sulfate-Polyacrylamide Gel Electrophoresis. Journal of Biological Chemistry 244: 44064412

Wehner P, Shnitsar I, Urlaub H \& Borchers A (2011) RACK1 is a novel interaction partner of PTK7 that is required for neural tube closure. Development 138: 1321-7

Wehrli M, Dougan ST, Caldwell K, O'Keefe L, Schwartz S, Vaizel-Ohayon D, Schejter E, Tomlinson a \& DiNardo S (2000) arrow encodes an LDL-receptor-related protein essential for Wingless signalling. Nature 407: 527-30

Wieschaus E \& Riggleman R (1987) Autonomous requirements for the segment polarity gene armadillo during Drosophila embryogenesis. Cell 49: 177-84

Willert K, Brink M, Wodarz A, Varmus H \& Nusse R (1997) Casein kinase 2 associates with and phosphorylates dishevelled. The EMBO Journal 16: 3089-96

Wilson C, Goberdhan DC \& Steller H (1993) Dror, a potential neurotrophic receptor gene, encodes a Drosophila homolog of the vertebrate Ror family of Trk-related receptor tyrosine kinases. Proceedings of the National Academy of Sciences of the United States of America 90: 7109-13

Winberg ML, Tamagnone L, Bai J, Comoglio PM, Montell D \& Goodman CS (2001) The transmembrane protein Off-track associates with Plexins and functions downstream of Semaphorin signaling during axon guidance. Neuron 32: 53-62

Wodarz a \& Nusse R (1998) Mechanisms of Wnt signaling in development. Annual Review of Cell and Developmental Biology 14: 59-88

Wodarz A (2008) Extraction and immunoblotting of proteins from embryos. Methods in Molecular Biology 420: 335-45

Wodarz A, Ramrath A, Grimm A \& Knust E (2000) Drosophila atypical protein kinase C associates with Bazooka and controls polarity of epithelia and neuroblasts. The Journal of Cell Biology 150: 1361-74 
Wolpert L, Jessel T, Lawrence P, Meyerowitz E, Robertson E \& Smith J (2007) Principles of Development

Wong GT, Gavin BJ \& Mcmahon AP (1994) Differential Transformation of Mammary Epithelial Cells by Wnt Genes. Molecular and Cellular Biology 14: 6278-6586

Wouda RR, Bansraj MRKS, Jong AWM De, Noordermeer JN \& Fradkin LG (2008) Src family kinases are required for WNT5 signaling through the Derailed / RYK receptor in the Drosophila embryonic central nervous system. Development 2287: 2277-2287

Yamamoto S, Nishimura O, Misaki K, Nishita M, Minami Y, Yonemura S, Tarui H \& Sasaki H (2008) Cthrc1 selectively activates the planar cell polarity pathway of Wnt signaling by stabilizing the Wnt-receptor complex. Developmental Cell 15: 23-36

Yanagawa S, Van Leeuwen F, Wodarz a, Klingensmith J \& Nusse R (1995) The dishevelled protein is modified by wingless signaling in Drosophila. Genes \& Development 9: 10871097

Yang-Snyder J, Miller JR, Brown JD, Lai CJ \& Moon RT (1996) A frizzled homolog functions in a vertebrate Wnt signaling pathway. Current Biology 6: 1302-6

Yao Y, Wu Y, Yin C, Ozawa R, Aigaki T, Wouda RR, Noordermeer JN, Fradkin LG \& Hing H (2007) Antagonistic roles of Wnt5 and the Drl receptor in patterning the Drosophila antennal lobe. Nature Neuroscience 10: 1423-32

Yen WW, Williams M, Periasamy A, Conaway M, Burdsal C, Keller R, Lu X \& Sutherland A (2009) PTK7 is essential for polarized cell motility and convergent extension during mouse gastrulation. Development 136: 2039-48

Yoda A, Oishi I \& Minami Y (2003) Expression and function of the Ror-family receptor tyrosine kinases during development: lessons from genetic analyses of nematodes, mice, and humans. Journal of Receptor and Signal Transduction 23: 1-15

Yoshikawa S, Mckinnon RD, Kokel M \& Thomas JB (2003) Wnt-mediated axon guidance via the Drosophila Derailed receptor. Nature 422: 3-8

Zallen JA (2007) Planar polarity and tissue morphogenesis. Cell 129: 1051-63

Zeng X, Tamai K, Doble B, Li S, Huang H, Habas R, Okamura H, Woodgett J \& He X (2005) A dual-kinase mechanism for Wnt co-receptor phosphorylation and activation. Nature 438: $873-7$

Zhang J \& Carthew RW (1998) Interactions between Wingless and DFz2 during Drosophila wing development. Development 125: 3075-85 


\section{APPENDIX}

Appendix 1: Crossing scheme to obtain an otk deletion by FLP-FRT mediated recombination.

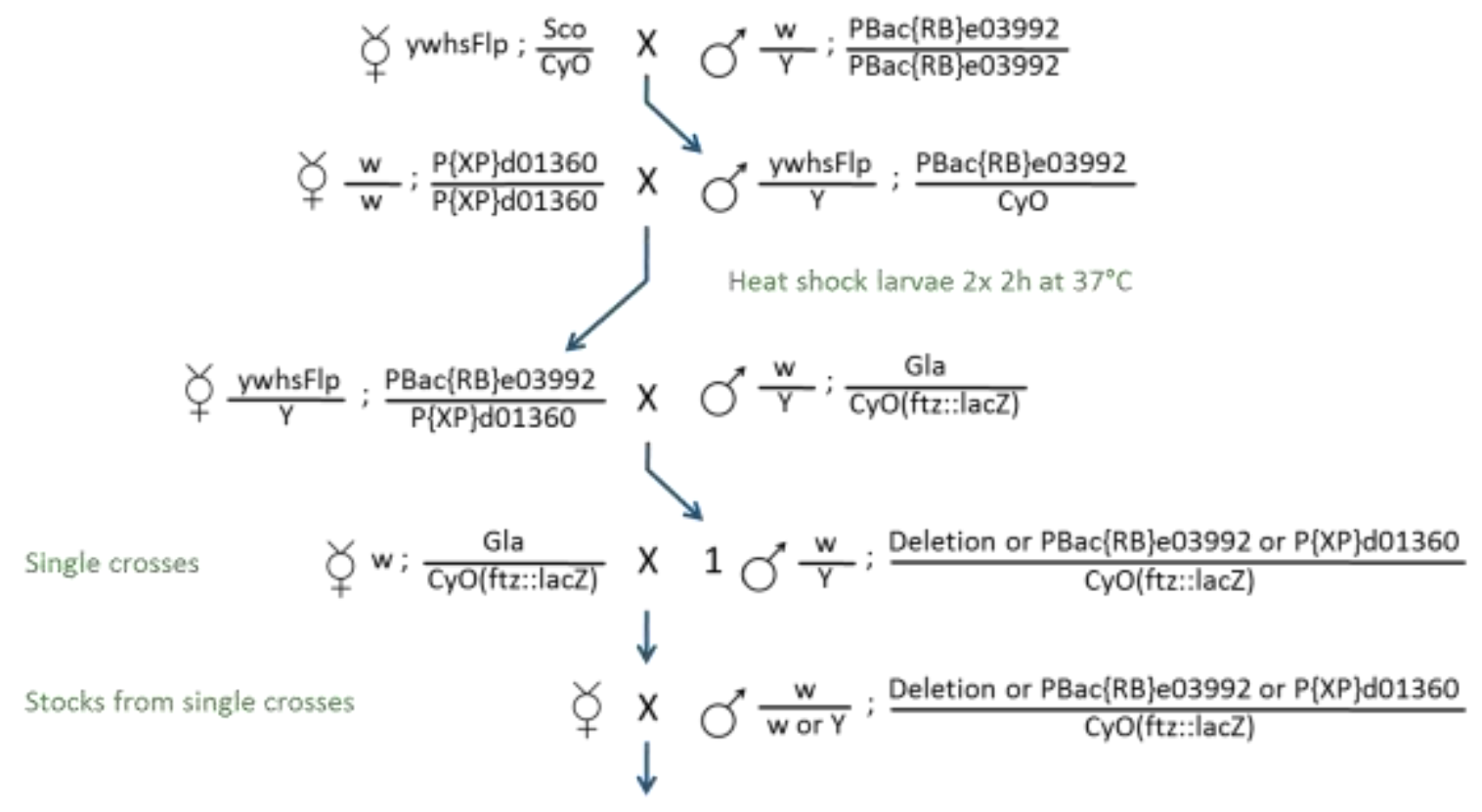

Screen stocks for lethality and succesful deletion

Appendix 2: Crossing scheme to obtain an otk2 deletion by FLP-FRT mediated recombination.

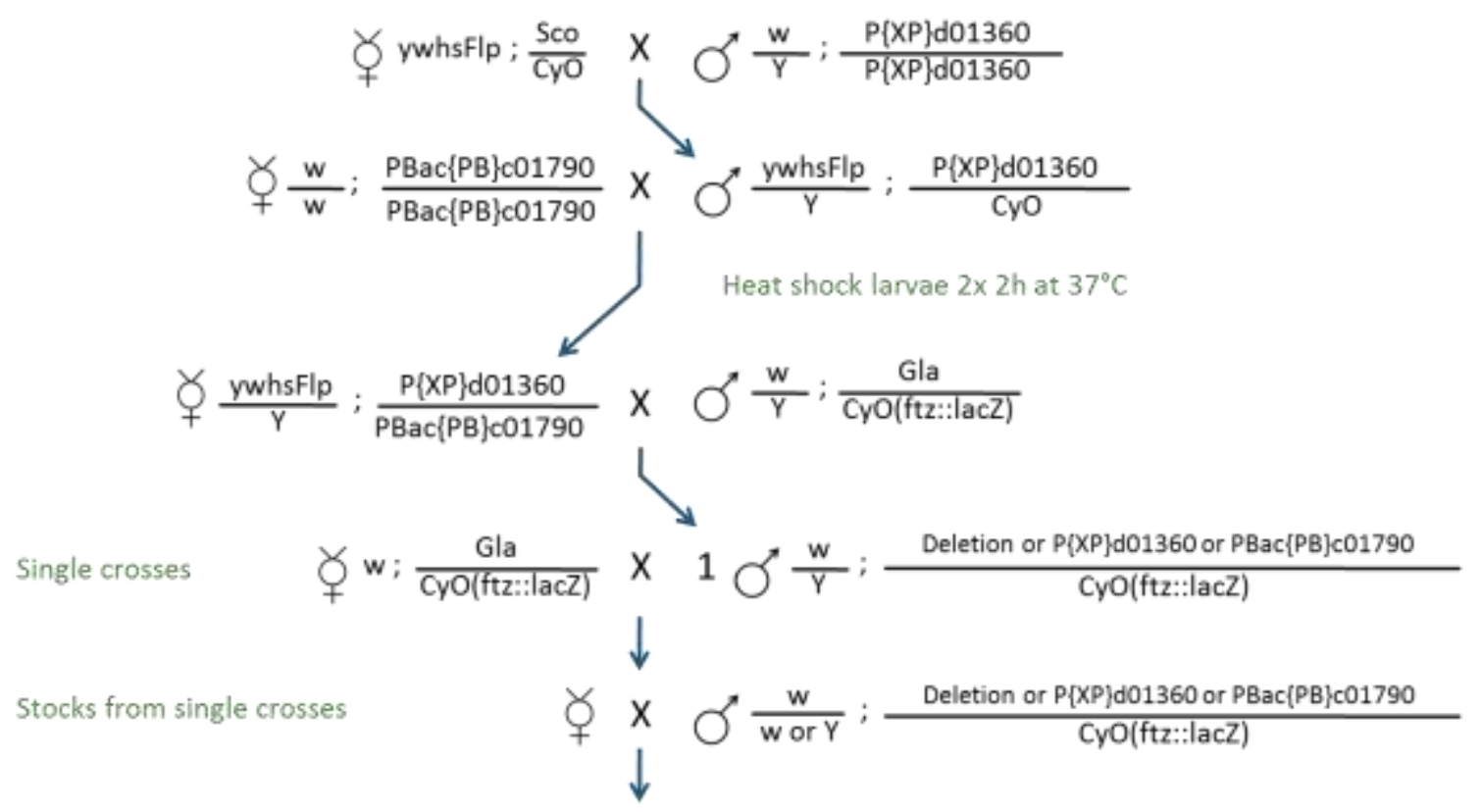

Screen stocks for lethality and succesful deletion 
Appendix 3: Crossing scheme to obtain an otk,otk2 deletion by FLP-FRT mediated recombination.

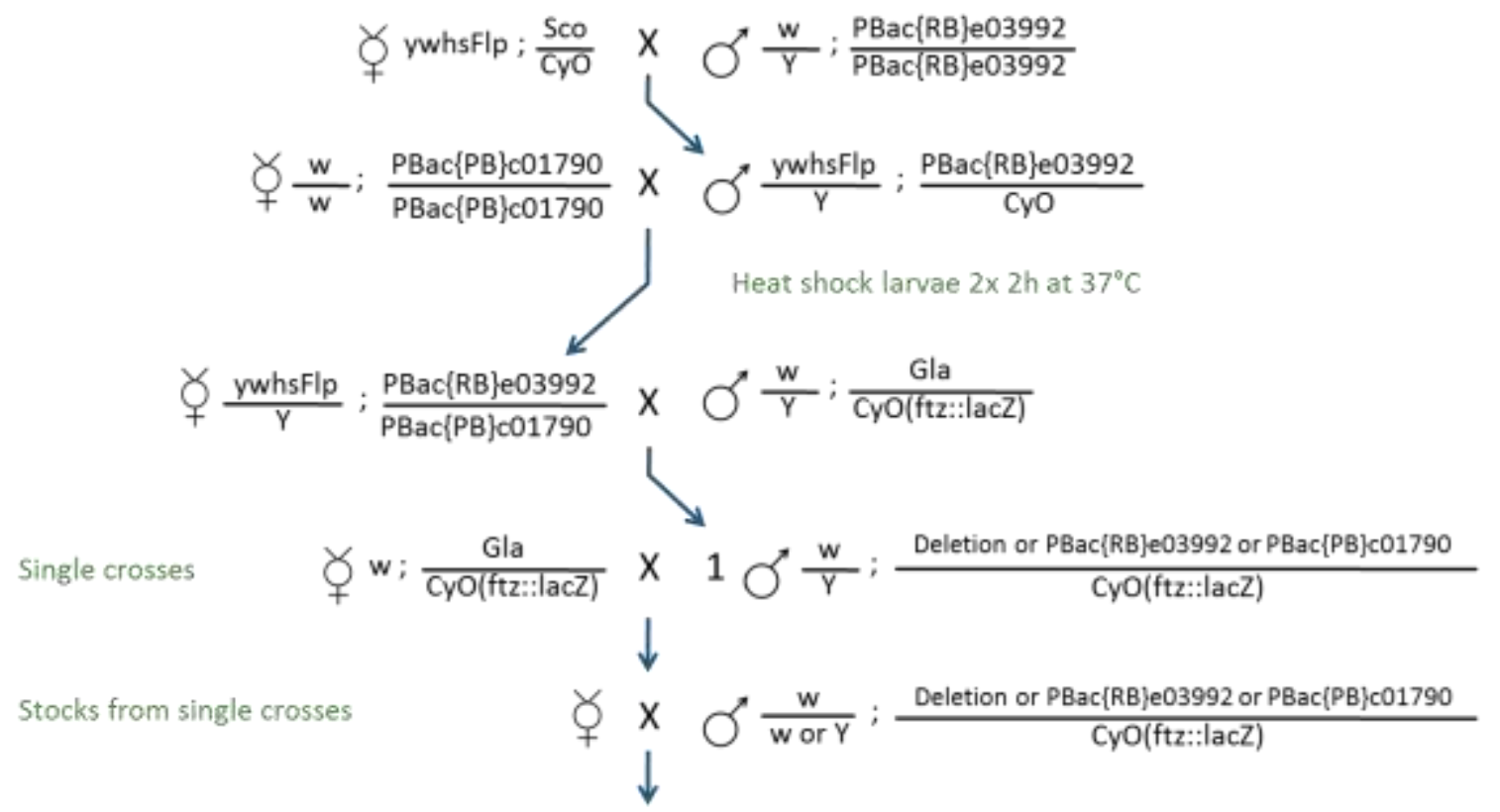

Screen stocks for lethality and succesful deletion 
Appendix 4: Sperm of otk,otk2 ${ }^{D 72}$ heterozygous mutant males enters the female reproductive tract. Reproductive apparatus from females after mating to adult males heterozygous mutant for otk,otk2 ${ }^{D 72}$ and carrying a ProtaminB-eGFP transgene. ut, uterus; sp, spermatheca; sv, seminal vesicle. Images were taken by Caroline Ripp. Scale bars $=100 \mu \mathrm{m}$.

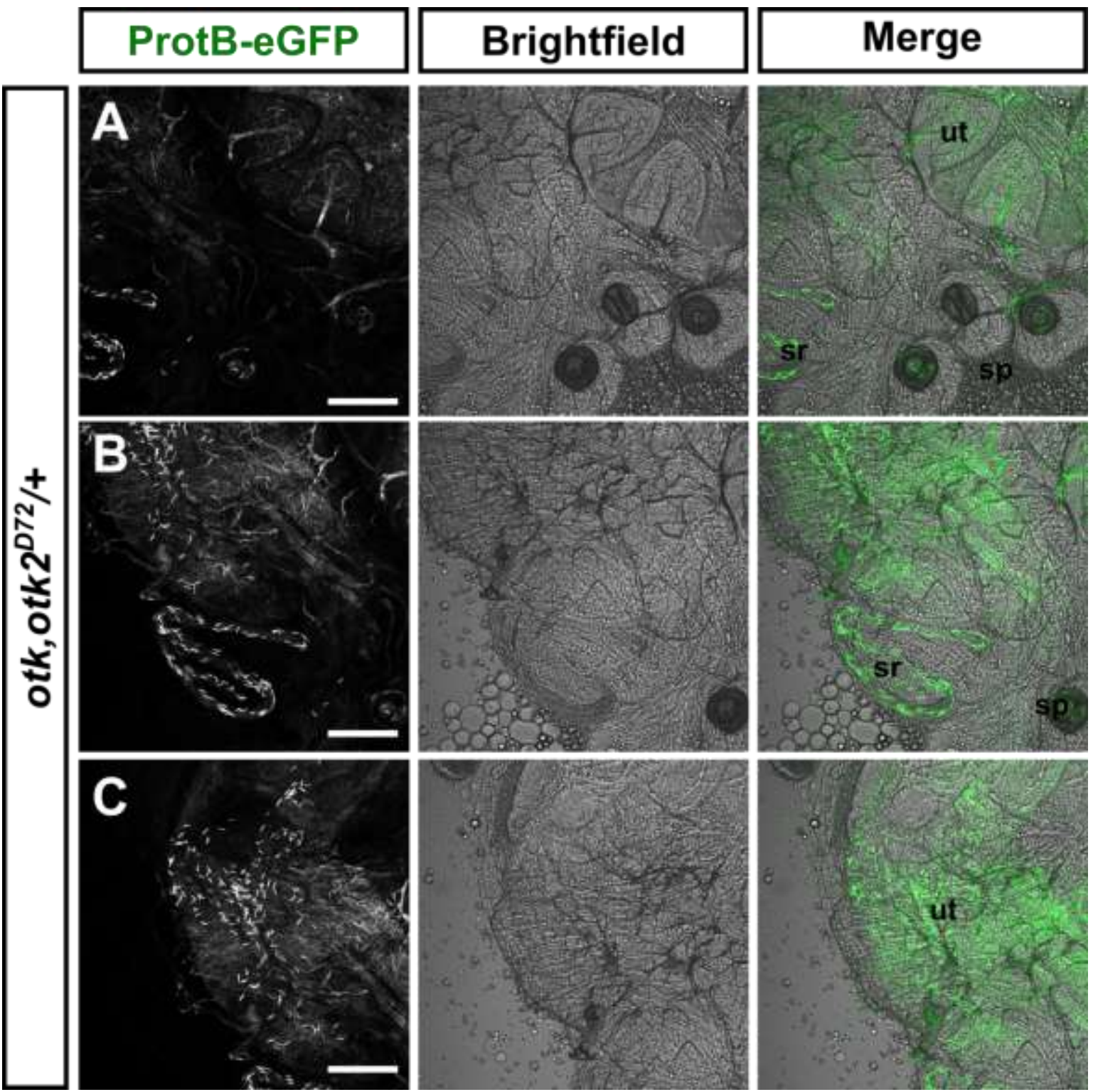

Appendix 5: Vector maps and genomic regions of Otk and 0tk2 (CD). 